\title{
An Extended Reynolds Equation Development with Applications to Fixed Geometry Bearings and Squeeze Film Dampers
}

\author{
A Dissertation Presented to the \\ Faculty of the School of Engineering and Applied Science \\ University of Virginia \\ In Partial Fulfillment \\ of the requirements for the Degree \\ Doctor of Philosophy
}

by

Saeid Dousti

December 2014 


\section{APPROVAL SHEET}

This dissertation is submitted in partial fulfillment of the requirements for the degree of

Doctor of Philosophy in Mechanical and Aerospace Engineering

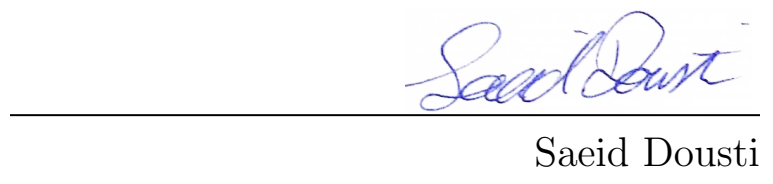

This dissertation has been read and approved by the Examining Committee:

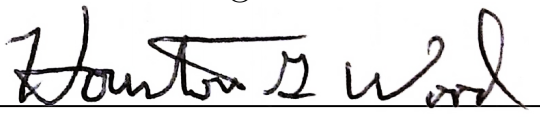

Houston G. Wood, Advisor
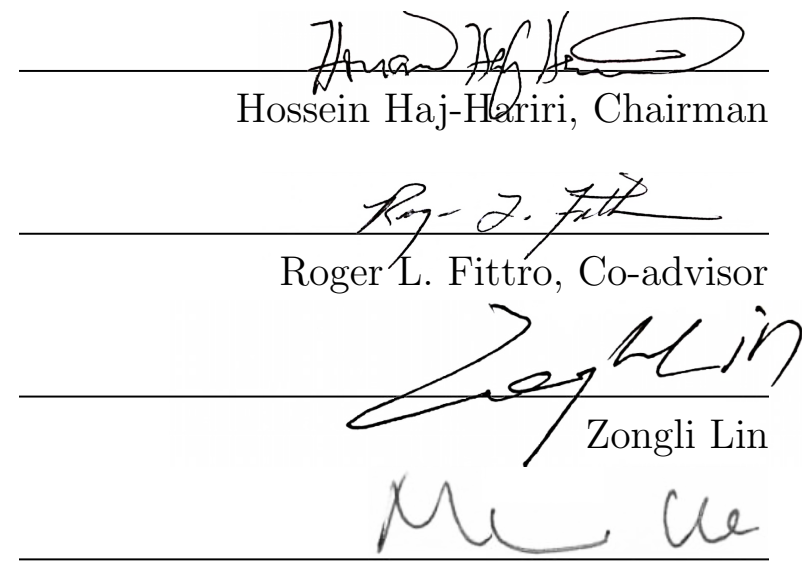

Minhui He

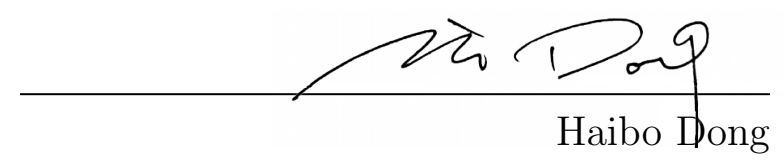

Accepted for the School of Engineering and Applied Science:

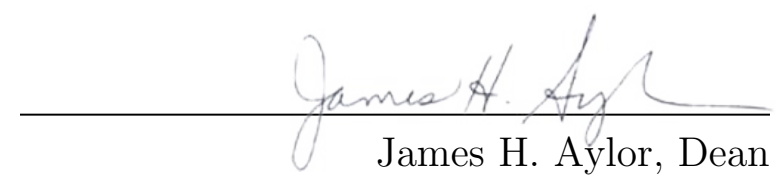

December 2014 
To my parents, Samad \& Arasta, and my brothers, Masoud \& Majid, I dedicate this $\mathrm{PhD}$ dissertation. 


\section{Acknowledgements}

I would like to express my sincere gratitude to Professor Paul Allaire. I personally feel that he was most instrumental in helping me obtain my $\mathrm{PhD}$. He has been a knowledgeable advisor, and a supportive friend throughout the exciting and difficult times during my stay at the University of Virginia.

I am deeply grateful to the Rotating Machinery and Controls (ROMAC) laboratory where I felt like home during my stay in Charlottesville. I would like to thank Professor Houston Wood and the committee members for their support and help. Special thanks to Roger Fittro whose help and guidance enabled me to finish this work. I appreciate all of my friends and colleagues: Jason Kaplan, Tim Dimond, Michael Branagan, Bradley Nichols and all other ROMAC people who have been very helpful. 


\section{Abstract}

Water lubricated bearings and squeeze film dampers exhibit large lubricant inertia forces on the order of viscous forces. To model these bearings, the traditional Reynolds equation is not adequate. An extended Reynolds equation is developed in this study which takes into account the turbulence and inertia effects: both convective and temporal. The most complete form of the temporal inertia effect model is developed and applies to the turbulent regime, consisting of primary and secondary temporal inertia terms. The convective inertia model follows Constantinescu's approach. The turbulence model is also based on Constantinescu's model and is tuned using CFD analysis.

To apply the proposed model to fixed geometry cylindrical water bearings, a computer code named "SLEEVEBRG" is developed. This program is also capable of satisfying the circumferential periodicity constraint associated with cylindrical bearings. The numerical results show significant convective and temporal inertia effects in water bearings. Convective inertia effects increase the load capacity of the bearing. The temporal inertia, resulting in effective added mass coefficients, exhibits destabilizing effects, while the convective inertia effects serve to improve stability of the bearing. The secondary temporal inertia reduces the added mass terms, thus increasing the stability of the bearing.

Squeeze film dampers are designed with numerous different configurations. Fea- 
tures such as supply and discharge holes, end seals, and grooves contribute into the dynamic characteristics of squeeze film dampers. Grooves are shown, contrary to previous perceptions, to generate a considerable amount of dynamic pressure. To capture their effects, an effective groove depth approach is adopted in this work. Additionally, an applicable extended Reynolds equation is developed which includes temporal inertia and also takes into account the contribution of the holes. A computer code named "MAXSFD" is developed accordingly. Four different configurations are analyzed and results are compared against experimentally obtained data. It is found that in general two distinct effective groove depths are required to match the experimental added mass and damping values. This indicates that different amounts of lubricant trapped in the groove contribute to the added mass and damping characteristics of squeeze film dampers. In open end squeeze film dampers, the ratio of the two effective grooves is nearly two, and for sealed squeeze film dampers the ratio is approximately one long and one and half for short SFD. The numerical observations prove that secondary temporal inertia plays a minor role in the determination of the added mass coefficients. The results are compared against an older squeeze film damper code SQFDAMP [1], which underpredicts the damping values and lacks the added mass prediction capability. 


\section{Contents}

\begin{tabular}{|ll}
\hline Acknowledgements & i
\end{tabular}

Abstract ii

Nomenclature vii

$\begin{array}{lll}1 & \text { Introduction } & 1\end{array}$

1.1 Overview. . . . . . . . . . . . . . . . . . . . 1

1.2 Literature Review . . . . . . . . . . . . . . . . . . . . . 6

$\begin{array}{lll}2 & \text { Hydrodynamic Bearing Equations } & 16\end{array}$

2.1 Kinematics of Radial Bearings . . . . . . . . . . . . . . . . . . . . . 16

2.2 Reynolds Equation For Incompressible Fluids . . . . . . . . . . . . . 23

$2.3 \quad$ Extended Reynolds Equation For Incompressible Fluids . . . . . . . . 25

2.3 .1 Constantinescue's method . . . . . . . . . . . . . . . 25

2.3 .2 The Modified Approach . . . . . . . . . . . . . . . . . . . . . 31

\begin{tabular}{lll}
3 & Solution Method & 33 \\
\hline
\end{tabular}

3.1 Galerkin Method . . . . . . . . . . . . . . . . . . . . . . . . 33

3.2 Rectangular Isoparametric Element . . . . . . . . . . . . . . . . . . . 38 
\begin{tabular}{|lll}
\hline & Fixed Geometry Sleeve Bearings & 42
\end{tabular}

4.1 Methodology and Governing Equations . . . . . . . . . . . . . . . 43

4.1 .1 Zeroth Order Equation . . . . . . . . . . . . . . 45

$4.1 .2 \quad$ Auxiliary Equation, Stiffness and Damping . . . . . . . . . . . 46

4.1.3 Auxiliary Equation, Temporal Inertia and Added Mass . . . . 47

4.1 .4 Rigid Rotor Stability . . . . . . . . . . . . . . . . . 51

4.2 SLEEVEBRG $\ldots \ldots \ldots \ldots$

4.3 Results . . . . . . . . . . . . . . . . . . . . . 56

$4.3 .1 \quad$ Water Bearing Pad Analysis . . . . . . . . . . . . . . . 56

4.3 .2 Ferron et al. Bearing . . . . . . . . . . . . . . . . . . . 64

4.3 .3 Water Bearing . . . . . . . . . . . . . . . . . . . 79

\begin{tabular}{|lll}
5 & Squeeze Film Dampers & 93
\end{tabular}

5.1 Geometry and Operation . . . . . . . . . . . . . . . . 95

5.1 .1 Groove $(\mathrm{s})$

$5.1 .2 \quad$ Orbit. . . . . . . . . . . . . . . . . . . . . . . . . . 98

5.2 Methodology and Governing Equations . . . . . . . . . . . . . . . . . 98

5.2 .1 Supply/Discharge Hole Models . . . . . . . . . . . . . . . . 100

5.2 .2 Axial End Models . . . . . . . . . . . . . . . . . . . . 103

5.3 MAXSFD . . . . . . . . . . . . . . . . . . . . . . 105

5.3 .1 Whirl Speed Dependent Approach . . . . . . . . . . . . . . . 106

$5.3 .2 \quad$ Whirl Speed Independent Approach . . . . . . . . . . . . . . . 107

5.4 Results . . . . . . . . . . . . . . . . . . . . . . . . . . . . . . 108

6 Conclusions and Recommendations 123

\begin{tabular}{lr}
\hline References & 129
\end{tabular} 


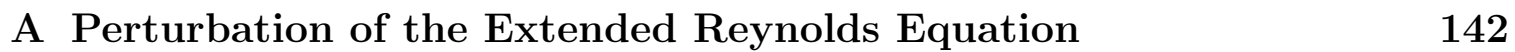

B Derivation of the General Reynolds Equation For An Isothermal Compressible Fluid

$\begin{array}{ll}\text { C Coefficient Identification for a CCO } & 155\end{array}$ 


\section{Nomenclature}

$\overrightarrow{\mathbf{a}}$ acceleration vector

c bearing clearance

$c_{p} \quad$ piston ring seal clearance in the seal groove

$d_{\text {eff }}$ effective groove depth

$e$ journal radial eccentricity with respect to bearing center

$h$ film thickness

$h_{p} \quad$ film thickness of the piston ring in its groove

$\hat{i}, \hat{j} \quad$ Cartesian coordinates, horizontal and vertical

$\hat{n}_{r}, \hat{n}_{\theta} \quad$ polar coordinates, radial and circumferential directions

$j=\sqrt{-1}$

$k \quad$ stiffness

$k$ a constant in Reichardt's eddy diffusivity equation

$k_{x}, k_{z} \quad$ Constantinescu's turbulence coefficients

$\vec{n}$ normal surface vector

$m_{o}, n_{o}$ experimentally obtained constants of Hirs' wall shear formula

$\overrightarrow{\mathbf{n}} \quad$ surface normal vector

$p$ pressure in lubricant film

$\tilde{p}$ approximate pressure solution of Reynolds equation

$p_{h} \quad$ supply or discharge hole pressure 
$p_{o}$ ambient pressure

$p_{s} \quad$ supply pressure

$p_{d} \quad$ discharge pressure

$q$ unit volumetric flow rate, $\left[L^{2} T^{-1}\right]$

$q_{l}$ end seal leakage unit volumetric flow rate

$r$ orbit radius size, $=\sqrt{X^{2}+Y^{2}}$

$s$ control volume surface

$t$ time

$u$ local fluid velocity along direction of rotation

$\overrightarrow{\mathbf{u}}$ general velocity vector

$u_{h}$ mean supply or discharge velocity $=\frac{Q_{h}}{A_{h}}$

$u^{\prime}$ fluctuating velocity in turbulent regime

$v$ volume of control volume

$v$ local fluid velocity in film thickness/radial direction

$v_{*} \quad$ wall velocity

$w$ local fluid velocity along axial direction

$w_{p}$ piston ring axial width

$x$ displacement

$x$ horizontal direction/displacement equivalent to circumferential direction/displacement

$x_{\text {pmax }}$ maximum pressure location in the circumferential direction

$y$ vertical direction/displacement equivalent to radial direction/displacement

$z$ axial direction/displacement

$\tilde{\mathbf{C}}$ fourth order material tensor

C damping

$C_{p}$ piston ring seal leakage coefficient

$C_{h}$ supply or discharge flow rate coefficient 
$D$ number of nodes in a pressure domain grid

$D_{h} \quad$ supply discharge hole diameter

$F$ force

$F_{X, Y}$ external horizontal and vertical forces

$F_{a} \quad$ bearing applied forces $(N)$

$F_{r}, F_{\theta}$ radial and tangential forces $(N)$

$F_{n, m}$ hydrodynamic force of the lubricant film, $n=i, o$ inner or outer film, and $m=x, y$ horizontal or vertical direction, respectively

$\overrightarrow{\mathbf{F}}$ load vector

$\overrightarrow{\mathbf{F}} \quad$ volumetric body force vector

$G_{r}$ groove ratio

$G_{\theta}, G_{z} \quad$ Reynolds equation coefficients

$I_{i j}$ direct or cross coupled convective inertia variable

J Jacobian matrix

K stiffness

K fluidity matrix

$K_{s} \quad$ centering spring stiffness

L lower triangular matrix of fluidity matrix

$L$ characteristic length

$L_{h} \quad$ supply/discharge hole length

$L_{y} \quad$ across the lubricant film characteristic length

$L_{x z} \quad$ along the film characteristic length

$N_{i}$ element shape functions

$\mathbf{N}$ shape function vector

$O_{s}, O_{b}$ bearing and shaft centers

$\vec{P}$ the vector of nodal pressure values 
$P_{u} \quad$ bearing nominal pressure

$Q_{x}, Q_{z}$ distribution coefficients of the time derivative of flow rate gradients in $x$ and $z$ directions

$R$ journal radius

$\overrightarrow{\mathbf{T}}$ traction vector

$T, S$ circumferential and radial acceleration components

$U$ surface velocity of slider or journal

$U, V$ circumferential and radial velocity components

$U_{a}$ averaged velocity of lubricant film

$U_{p}, W_{p}$ averaged velocity of lubricant film's Poiseuille flow in $x$ and $z$ directions

$U_{2}, W_{2}$ top surface velocity of lubricant film in $x$ and $z$ directions

$U_{1}, W_{1}$ bottom surface velocity of lubricant film in $x$ and $z$ directions

$\mathbf{U} \quad$ upper triangular matrix of fluidity matrix

$\hat{X}, \hat{Y} \quad$ global Cartesian coordinates

$X, Y$ relative horizontal and vertical displacements of journal

$X_{b}, Y_{b}$ horizontal and vertical displacements of bearing housing

$X_{j}, Y_{j}$ horizontal and vertical displacements of journal

$\overrightarrow{\mathbf{V}} \quad$ velocity vector

$W$ axial length of element

$W \quad$ surface velocity in axial direction

$W_{s}, W_{b}$ weight of the shaft, and the bush in floating bush bearings

$\vec{V} \quad$ velocity vector

$V \quad$ lubricant general velocity

$V$ circumferential length of element

$\alpha$ eccentricity angle 
$\alpha, \beta, \gamma$ coefficients of Constantinescu's formula for inertia variables

$\alpha, \beta \quad$ real and imaginary part of eigenvalues

$\delta \quad \log$ decrement in stability analysis

$\delta \quad$ Kronecker delta

$\delta^{+}$a constant in Reichardt's eddy diffusivity equation

$\epsilon$ shear strain

$\xi, \eta$ bearing Cartesian coordinates

$\kappa$ eddy viscosity model constant

$\epsilon$ the empirical convective inertia constant

$\theta$ circumferential direction and variable

$\mu$ lubricant viscosity

$\rho$ lubricant density

$\varepsilon_{m} \quad$ eddy diffusivity

$\nu$ kinematic viscosity

$\tilde{\sigma} \quad$ stress tensor

$\phi \quad$ attitude angle, angle of line of journal and bearing centers with $x$ coordinate

$\phi$ field variable

$\lambda$ eigenvalue

$\lambda, \mu, \gamma \quad$ Lamé constants

$\varepsilon$ journal eccentricity ratio

$\vec{\Phi}_{e} \quad$ element field vector

$\tau$ viscous shear stress

$\tau$ volume element

$\tau_{w} \quad$ wall shear stress

$\tau^{\prime}$ Reynolds shear stress in turbulent regime

$\omega$ rotational speed 
$\Omega \quad$ whirl speed

$\omega_{b}$ rotational speed of the bearing housing

$\zeta, \varrho$ perturbation variables

$\Delta$ dilatation or trace of the strain tensor

$\Lambda$ characteristic time

$\bar{x}=\frac{x}{L_{x z}}$, dimensionless horizontal/circumferential displacement

$\bar{y}=\frac{y}{L_{y}}$, dimensionless vertical/radial displacement

$\bar{z}=\frac{z}{L_{x z}}$, dimensionless axial displacement

$\bar{u}=\frac{u}{U}$, dimensionless horizontal/circumferential velocity

$\bar{v}=\frac{v}{U} \frac{L_{x z}}{L_{y}}$, dimensionless vertical/radial displacement

$\bar{w}=\frac{w}{U}$, dimensionless axial displacement

$\bar{p}=\frac{p L_{y}}{\mu U} \frac{L_{y}}{L_{x y}}$, dimensionless pressure

$y^{+}=\frac{y v_{*}}{\nu}$, nondimensional distance from wall

$\mathbf{R e}=\frac{\rho U c}{\mu}$, Reynolds number

$\mathbf{R e}_{\text {hole }}$ supply, discharge hole Reynolds number

$\mathbf{R e}^{*}=\boldsymbol{R e} \frac{c}{R}$, reduced Reynolds number

$\Lambda^{*}=\frac{\rho c^{2} \Lambda}{\mu}$, reduced flow frequency number

$\bar{t}=\Lambda t$, non-dimensional time 


\section{List of Figures}

1.1 Thin lubricant film, $[3]$. . . . . . . . . . . . . . . . . . . . . 2

1.2 Schematic of a squeeze film damper [11] . . . . . . . . . . . . . . . . 4

1.3 Cut-away view of squeeze film damper with lubrication holes and grooves

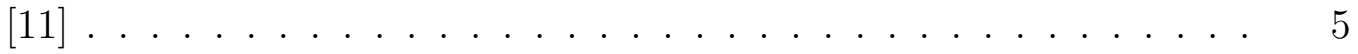

2.1 Squeeze motion in thin lubricant film, [3] . . . . . . . . . . . . . 18

$2.2 \quad$ Eccentric journal in radial bearing . . . . . . . . . . . . . . . . . . . . 19

2.3 Flow Between Plates: a Superposition of Poiseuille and Couette Flows 27

3.1 Pressure domain and boundaries . . . . . . . . . . . . . . . . . . . 34

3.2 Global and local isoprametric elements . . . . . . . . . . . . . . . . . 38

4.1 sleeve bearing with no axial groove . . . . . . . . . . . . . . . . . 43

4.2 squeeze motion $\ldots \ldots \ldots$. . . . . . . . . . . . . . . . . . . 48

4.3 Cylindrical water bearing . . . . . . . . . . . . . . . 53

4.4 Structure of computer code SLEEVEBRG . . . . . . . . . . . . . . 54

4.5 Erroneous jump at the boundaries between cavitation and uncavitated regions . . . . . . . . . . . . . . . 56

4.6 CFD model of a single pad of a tilting pad water bearing by Armentrout et al. [50] . . . . . . . . . . . . . . . . . . . . . 57 
4.7 Pressure profile of the mid-plane of a single pad. Laminar regime

without convective inertia. CFD results from [50] . . . . . . . . . . . 59

4.8 Pressure profile of the mid-plane of a single pad. Laminar regime with convective inertia. SLEEVEBRG results based on laminar and turbulent convective inertia models. CFD results from [50] . . . . . . 60

4.9 Correction to the inverse of Sommerfeld number, Fig. (1) of [14] . . . 61

4.10 Pressure profile of the mid-plane of a single pad operating in the tur-

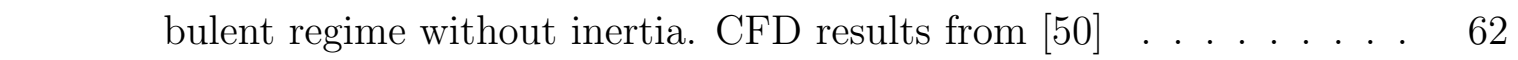

4.11 Pressure profile of the mid-plane of a single pad. Turbulent regime with inertia. SLEEVEBRG results based on laminar and turbulent convective inertia models. CFD results from [50] . . . . . . . . . . . 63

4.12 Single axial groove Ferron et al. bearing . . . . . . . . . . . . . . 66

4.13 Comparison of the mid-span pressure profile of Ferron et al. bearing

from experiment, thermohydordynamic analysis of MAXBRG (by [42]) and THBRG, and isothermal hydordynamic analysis of SLEEVEBRG at 2000(rpm), 4000(N) load, and $\mu=0.018($ Pa.s $)$. . . . . . . . 67

4.14 Variation of eccentricity ratio vs. applied vertical load, $\omega=2000(\mathrm{rpm}) \quad 68$

4.15 Steady state operation of Ferron et al. bearing with a $200(K g)$ load , and $\mu=0.018($ Pa.s $)$ for SLEEVEBRG . . . . . . . . . . . . . . 69

4.16 Principle stiffness, Ferron et al. bearing with $200(K g)$ vertical load and $2000(\mathrm{rpm})<\omega<20000(\mathrm{rpm})$ rotational speed . . . . . . . . . . . . . 71

4.17 Cross-coupled stiffness, Ferron et al. bearing with $200(\mathrm{Kg})$ vertical load and $2000($ rpm $)<\omega<20000($ rpm $)$ rotational speed . . . . . . . 72

4.18 Principle damping, Ferron et al. bearing with $200(K g)$ vertical load and $2000(\mathrm{rpm})<\omega<20000(\mathrm{rpm})$ rotational speed . . . . . . . . . 73 
4.19 Cross-coupled damping, Ferron et al. bearing with $200(K g)$ vertical load and $2000(\mathrm{rpm})<\omega<20000(\mathrm{rpm})$ rotational speed . . . . . . . 74

4.20 Principle added mass, Ferron et al. bearing with $200(K g)$ vertical load

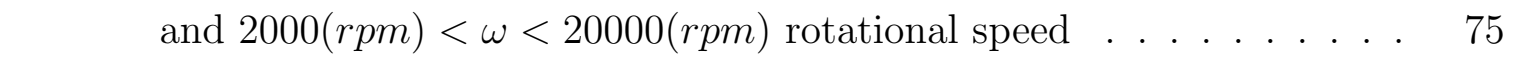

4.21 Cross-coupled added mass, Ferron et al. bearing with $200(K g)$ vertical load and 2000(rpm) $<\omega<20000($ rpm $)$ rotational speed . . . . . . . 76

4.22 Stability of Ferron et al. bearing with $200(\mathrm{Kg})$ vertical load and $2000(\mathrm{rpm})<$

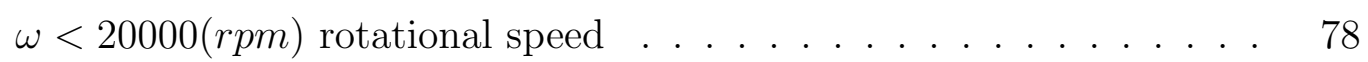

4.23 Steady state operation of water bearing with a $1500(\mathrm{Kg})$ vertical load and $500<\omega<12000(\mathrm{rpm})$ operational speed range . . . . . . . . . . 83

4.24 Principle stiffness, water bearing with $1500(\mathrm{Kg})$ vertical load and $500<$ $\omega<12000($ rpm $)$ operational speed range . . . . . . . . . . . . . . . . 84

4.25 Cross-coupled stiffness, water bearing with $1500(\mathrm{Kg})$ vertical load and $500<\omega<12000(\mathrm{rpm})$ operational speed range . . . . . . . . . . . . 85

4.26 Principle damping, water bearing with $1500(\mathrm{Kg})$ vertical load and

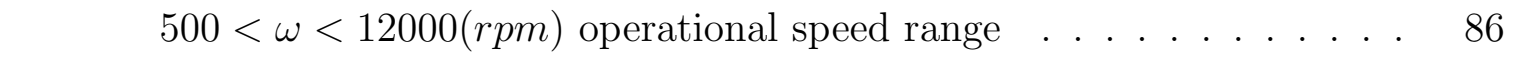

4.27 Cross-coupled damping, water bearing with 1500(Kg) vertical load and $500<\omega<12000($ rpm $)$ operational speed range . . . . . . . . . . . . 87

4.28 Horizontal principle added mass, water bearing with $1500(\mathrm{Kg})$ vertical load and $500<\omega<12000(\mathrm{rpm})$ operational speed range . . . . . . . 88

4.29 Vertical principle added mass, water bearing with $1500(\mathrm{Kg})$ vertical

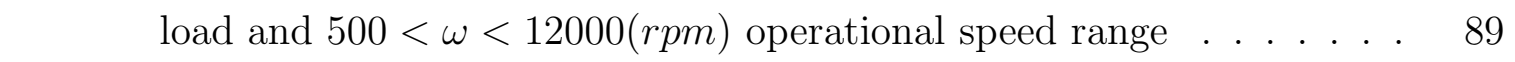

4.30 Cross-coupled added mass, water bearing with $1500(\mathrm{Kg})$ vertical load and $500<\omega<12000(\mathrm{rpm})$ operational speed range . . . . . . . . . . 90

4.31 Cross-coupled added mass, water bearing with $1500(\mathrm{Kg})$ vertical load and $500<\omega<12000($ rpm $)$ operational speed range . . . . . . . . . . 91 
4.32 Stability of a water bearing with $1500(\mathrm{Kg})$ vertical load and $500<\omega<$ $12000(\mathrm{rpm})$ operational speed range . . . . . . . . . . . . . 92

5.1 Different configurations of squeeze film dampers, axial cutaway view. $\quad 94$

5.2 Traditional perception of central groove; zero dynamic pressure in groove [1] . . . . . . . . . . . . . . . . . . . . . . . . . . . . . . . . . . . . . . . . 95

5.3 The streamlines of the flow in the groove regions of SFD . . . . . . . 96

5.4 Piston ring . . . . . . . . . . . . . . . . . . . . . . . 103

5.5 Mesh grid in MAXSFD . . . . . . . . . . . . . . . . . . . . 104

5.6 Circular centered orbit . . . . . . . . . . . . . . . . . . . . . . . . . 105

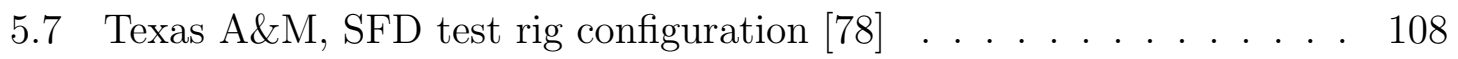

5.8 Short open end SFD, linear coefficients vs. whirl amplitude ratio, results from MAXSFD, SQFDAMP and test data from [78] . . . . 109

5.9 Long open end SFD, linear coefficients vs. whirl amplitude ratio, results from MAXSFD, SQFDAMP and test data from [78] . . . . 110

5.10 Short and long open end SFD, linear coefficients vs. whirl amplitude ratio with $d_{e f f}=9.5(\mathrm{~mm})$, results from MAXSFD, SQFDAMP and test data from $[78] \ldots \ldots \ldots$

5.11 Short sealed end SFD, linear coefficients vs. whirl amplitude ratio, results from MAXSFD, SQFDAMP and test data from [76] . . . 115

5.12 Long sealed end SFD, linear coefficients vs. whirl amplitude ratio, results from MAXSFD, SQFDAMP and test data from [76] . . . 116

5.13 Short and long sealed end SFD, linear coefficients vs. whirl amplitude ratio with $d_{e f f}=9.5(\mathrm{~mm})$, results from MAXSFD, SQFDAMP and test data from $[76]$. . . . . . . . . . . . . . . . . . . . . . . . . . . . 117 
5.14 Pressure profile of short open end SFD, $G_{r}=2.5, \frac{r}{c}=0.054, \Omega=$ $1000(\mathrm{rpm}) \ldots \ldots \ldots \ldots \ldots \ldots \ldots$

5.15 Pressure profile of short sealed end SFD, $G_{r}=4, \frac{r}{c}=0.054, \Omega=$ $1000(\mathrm{rpm}) \ldots \ldots \ldots \ldots \ldots \ldots \ldots$

5.16 Effect of secondary inertia models on the added mass coefficient for fixed $G_{r}$ values

B.1 Force acting on a small volume element . . . . . . . . . . . . . . . . . 147

B.2 Thin Lubricant Film, $[3]$. . . . . . . . . . . . . . . . . . . . . . 151

C.1 Circular centered orbit, CCO . . . . . . . . . . . . 156 
xviii

\section{List of Tables}

$4.1 \quad$ Water tilting pad bearing [50] . . . . . . . . . . . . . . . . . 58

4.2 Ferron et al. bearing characteristics [93] . . . . . . . . . . . . . 65

4.3 Water Bearing . . . . . . . . . . . . . . . . . . . . . . . 79

5.1 Texas A\&M test rig characteristics [78, 76] . . . . . . . . . . . . . . . 112

$5.2 \quad$ Optimum effective groove depth ratio to predict linear coefficients . . 118 


\section{Chapter 1}

\section{Introduction}

\subsection{Overview}

In 1886, Osborne Reynolds in his historic paper established a theory to address thin film hydrodynamic lubrication [2]. A thin lubricant film is shown in Fig. (1.1). He formulated his equation based on the following assumptions:

- The lubricant is Newtonian and incompressible.

- Gravitational forces are negligible compared to the hydraulic forces.

- The lubricating film is thin. Therefore, the main velocity gradient occurs across the film, the film curvature can be neglected and pressure across the film is constant.

- The lubricant sticks to the surfaces that it is in contact with, i.e., there is no relative velocity at the interface of the lubricant and surface.

- The bearing operates in laminar regime.

- The lubricant convective and temporal inertia terms are neglected. 


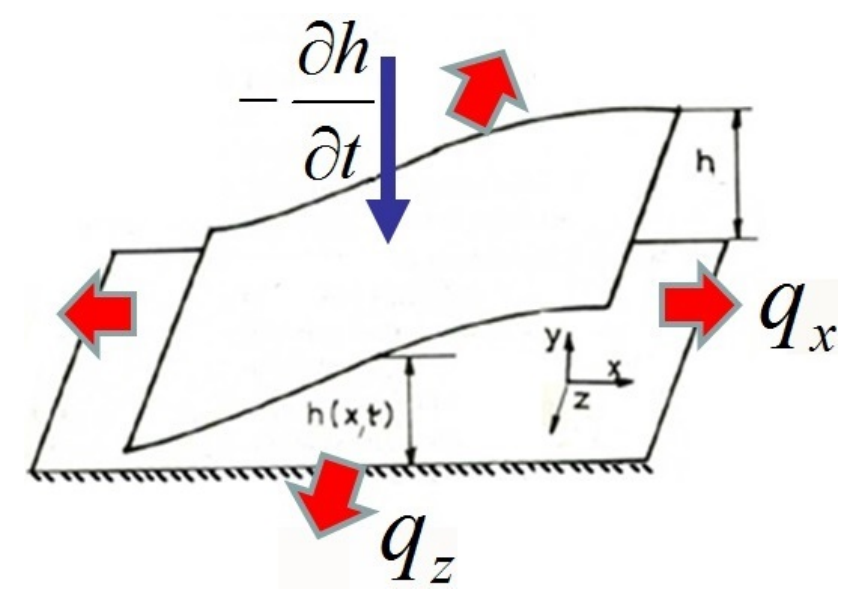

Figure 1.1: Thin lubricant film, [3]

The direct consequences of the aforementioned assumptions are a constant pressure profile and a parabolic component in the velocity variation across the lubricant film. Substitution of the velocity functions into the continuity equation and integration across the film results in the classic Reynolds equation [2] as

$$
\frac{\partial}{\partial x}\left(\frac{h^{3}}{\mu} \frac{\partial p}{\partial x}\right)+\frac{\partial}{\partial z}\left(\frac{h^{3}}{\mu} \frac{\partial p}{\partial z}\right)=6 U \frac{\partial h}{\partial x}+12 \frac{\partial h}{\partial t}
$$

In this equation, the main mechanism to produce pressure is viscous shearing, which is represented by $6 U \frac{\partial h}{\partial x}$ in the right hand side of the Reynolds equation. Viscous shearing results in stiffness properties in the bearings. The squeeze velocity, denoted by $12 \frac{\partial h}{\partial t}$, is the second mechanism to generate hydrodynamic pressure. Squeeze velocity is the damping mechanism of fluid film bearings. Thus, in a linearized terminology, fluid film bearing reaction forces can be represented as $F=-K x-C \dot{x}$. Since all the inertia terms are neglected, this theory is not capable of predicting the added mass coefficients of the bearing. While this theory has demonstrated good agreement with experiments for applications with low Reynolds numbers, deviations have been observed for cases with higher Reynolds numbers (e.g., high operating speeds, large 
clearance, etc.). Despite the ubiquitous utilization of this equation in lubrication applications, since the early 20th century, various researchers have attempted to extend this theory specifically by accounting for turbulence and inertia effects in high Reynolds number applications. Two main applications considered in this study are water bearings and squeeze film dampers (SFD). These two bearing types exemplify a class of applications where the traditional Reynolds equation is not adequate to accurately predict system behavior. In these applications, operating Reynolds numbers are usually high and inertia effects and turbulence are potentially significant and should, therefore, be included in the analysis.

Water Bearings: Environmental concerns about oil and grease lubricated bearings along with the practicality of oil based lubricants in some applications, e.g., submarines and sub-sea apparatus, have brought about an increase in the use of water-lubricated bearings. Many applications, including stern tube bearings on ships, submarines and small crafts, hydroelectric power plants and pumps use water bearings [4]. These bearings are manufactured in various designs. While some use rubber pads, others are simply journal bearings with axial or even spiral grooves. These designs are typically used for long bearings with relatively large $L / D$ ratios. The design concerns of these bearings are beyond the scope of this study and only the simple full cylindrical water bearings with no grooves and $L / D \sim 1$ are addressed here. The main concern in water bearings is the low viscosity of water, compared to oil, that increases $\mathbf{R e}=\frac{\rho U c}{\mu}$ and $\mathbf{R e}^{*}=\mathbf{R e} \frac{c}{R}$ drastically and therefore makes them prone to turbulence and fluid inertia effects. In other words, the linear approximation of the fluid film reaction forces via the $K$ and $C$ parameters, as suggested in the traditional Reynolds equation, is not adequate and should be amended to include lubricant added mass (i.e., $F=-K x-C \dot{x}-M \ddot{x}$ ). In addition, the $K$ and $C$ coefficients should be calculated with the turbulence effects included. 


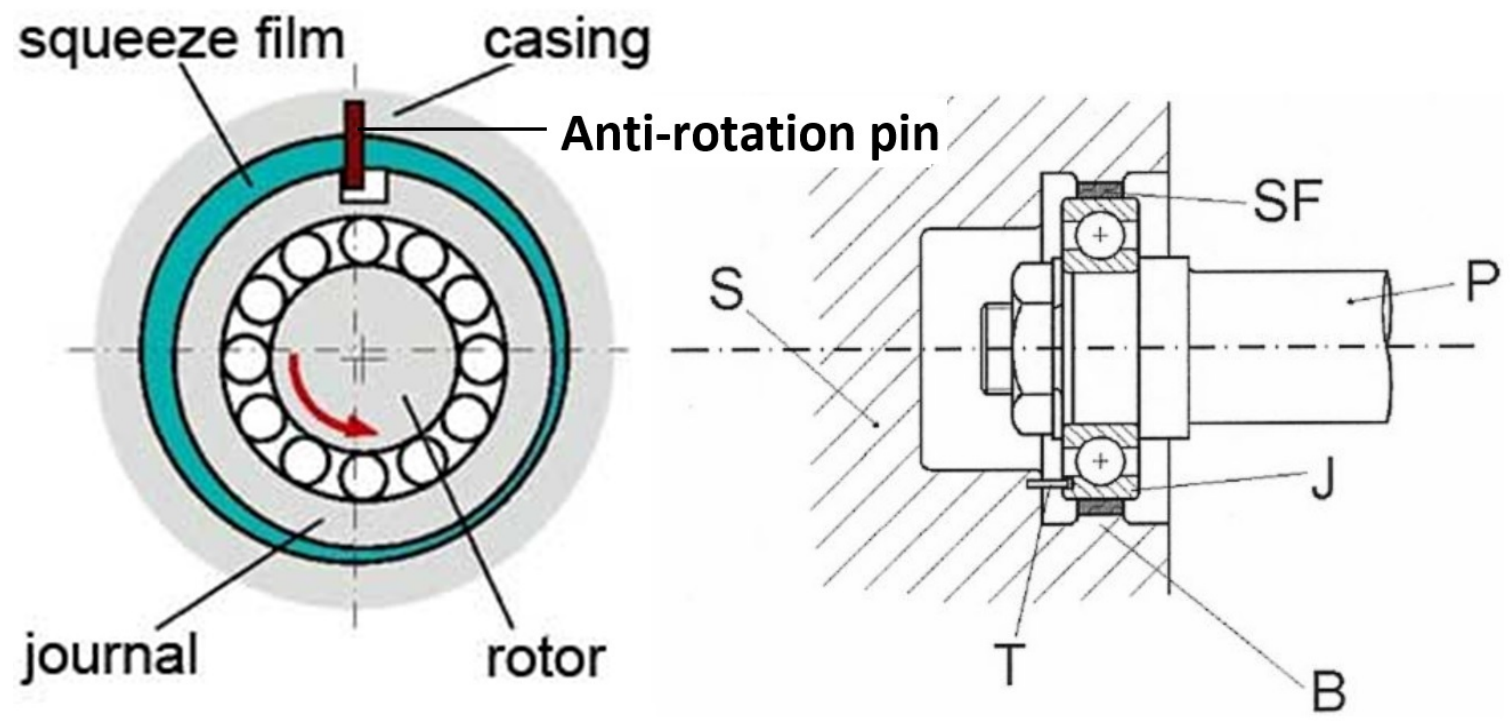

Figure 1.2: Schematic of a squeeze film damper [11]

Squeeze Film Dampers: Squeeze film dampers (SFDs) have also been reported to demonstrate significant inertia effects [5, 6, 7, 8, 9, 10]. SFDs are the shock absorbers of rotating machinery, especially for those utilizing rolling element bearings. SFDs are comprised of an annular thin film of lubricant between two non-rotating surfaces. Although, SFDs are often used to improve the stability of roller element bearings, they can also support rotating equipment with fluid film bearings. The inner surface (journal) is the outer race or housing of the bearing and the outer surface is the housing of the SFD. An anti-rotation pin prevents journal rotation which results in SFDs exhibiting no steady state stiffness properties Fig. 1.2. Thus, vibration in a machine activates the SFD and induces a dynamic journal whirling (precessional motion) orbit that squeezes the oil film, which subsequently generates hydrodynamic pressures on the whirling surface. Significant fluid inertia forces are observed in squeeze film dampers since they often operate with larger $\mathbf{R e}^{*}$ than found in typical journal bearings. 


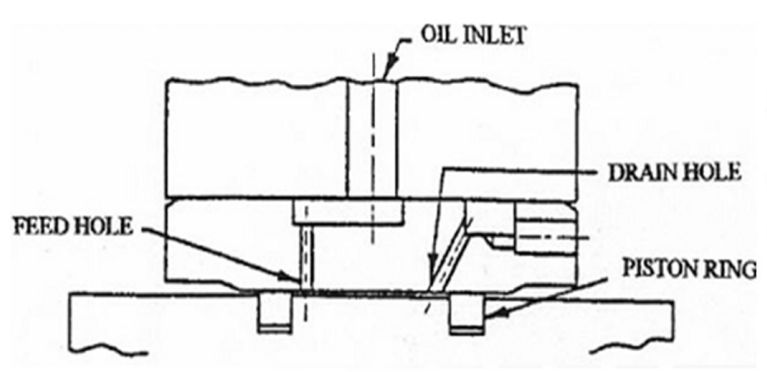

(a)

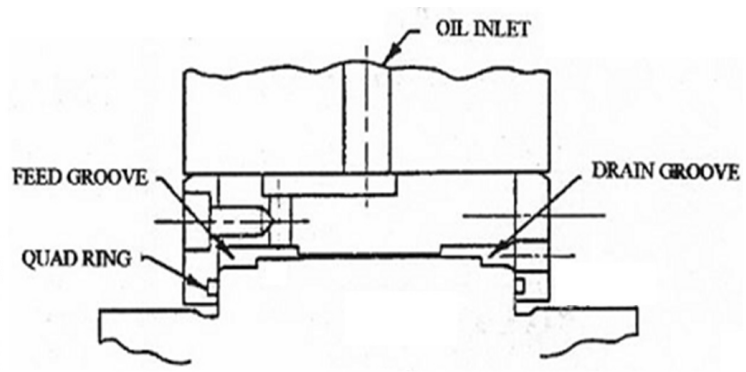

(b)

Figure 1.3: Cut-away view of squeeze film damper with lubrication holes and grooves 11

SFD geometries take on a variety of forms, see Fig. (1.3). Feeding (or supply) holes provide lubricant and usually fill an annular groove from which oil seeps into the damper land(s). In a reverse mechanism, sometimes lubricant is collected in a discharge groove and drained through discharge holes. Grooves are traditionally thought to provide supply/discharge pressure and isolate the dynamic interactions of the adjacent damper lands from each other. In some designs, SFDs with end seals incorporate grooves at the sides of the damper land(s) to prevent air ingestion to the damper. These grooves provide an oil plenum that prevents the ingestion of air into the damper land and minimizes cavitation [11]. Supply and discharge holes and their arrangements also contribute to the dynamics of SFDs. In simpler designs, the axial sides of the SFD open to ambient pressure and the lubricant leaks freely from the axial ends. To reduce the flow rate and increase the damping capacity of a SFD, piston or O-ring seals can be incorporated into the design. In the past half a century, extensive studies have attempted to model the dynamics of SFDs, including their inertia effects [11]; however, a comprehensive model which takes into account all the detailed design aspects listed above has yet to be developed.

In this study, three general goals are pursued. First, an effective extended Reynolds equation with the inclusion of inertia and turbulent effects is developed. Subsequently, 
this theory is applied to water journal bearings and squeeze film dampers.

\subsection{Literature Review}

In order to improve the applicability of Reynolds equation to high Reynolds number applications, modifications to this equation should include three types of effects: temporal inertia effects, convective inertia effects, and turbulence. By following the nondimensionalization method introduced by Szeri [12], the continuity and NavierStokes momentum equations are expressed as

$$
\begin{aligned}
& \frac{\partial \bar{u}}{\partial \bar{x}}+\frac{\partial \bar{v}}{\partial \bar{y}}+\frac{\partial \bar{w}}{\partial \bar{z}}=0 \\
& \boldsymbol{\Lambda}^{*} \frac{\partial \bar{u}}{\partial \bar{t}}+\mathbf{R e}^{*}\left(\bar{u} \frac{\partial \bar{u}}{\partial \bar{x}}+\bar{v} \frac{\partial \bar{u}}{\partial \bar{y}}+\bar{w} \frac{\partial \bar{u}}{\partial \bar{z}}\right)=-\frac{\partial \bar{p}}{\partial \bar{x}}+\frac{\partial^{2} \bar{u}}{\partial \bar{y}^{2}} \\
& \boldsymbol{\Lambda}^{*}\left(\frac{L_{y}}{L_{x z}}\right)^{2} \frac{\partial \bar{v}}{\partial \bar{t}}+\mathbf{R e}^{*}\left(\frac{L_{y}}{L_{x z}}\right)^{2}\left(\bar{u} \frac{\partial \bar{v}}{\partial \bar{x}}+\bar{v} \frac{\partial \bar{v}}{\partial \bar{y}}+\bar{w} \frac{\partial \bar{v}}{\partial \bar{z}}\right) \\
& =-\frac{\partial \bar{p}}{\partial \bar{y}}+\left(\frac{L_{y}}{L_{x z}}\right)^{2} \frac{\partial^{2} \bar{v}}{\partial \bar{y}^{2}} \\
& \boldsymbol{\Lambda}^{*} \frac{\partial \bar{w}}{\partial \bar{t}}+\mathbf{R e}^{*}\left(\bar{u} \frac{\partial \bar{w}}{\partial \bar{x}}+\bar{v} \frac{\partial \bar{w}}{\partial \bar{y}}+\bar{w} \frac{\partial \bar{w}}{\partial \bar{z}}\right)=-\frac{\partial \bar{p}}{\partial \bar{z}}+\frac{\partial^{2} \bar{w}}{\partial \bar{y}^{2}}
\end{aligned}
$$

In Eq. 1.4 , the order of the film thickness ratio is $O\left(\frac{L_{y}}{L_{x z}}\right) \approx 10^{-3}$, which means all the viscous and inertia terms are negligible and therefore this equation reduces to

$$
\frac{\partial \bar{p}}{\partial \bar{y}}=0
$$

In other words, the pressure gradient across the film is insignificant, as was originally assumed by Reynolds. The reduced flow frequency number, $\Lambda^{*}=\frac{\rho \Lambda L_{y}^{2}}{\mu}$, is the coefficient of the temporal inertia terms, and the reduced Reynolds number, $\mathbf{R e}^{*}=\frac{\rho \omega L_{y}^{2}}{\mu}$, is the coefficient of the convective terms. These two nondimensional coefficients reveal 
the magnitude of the inertia terms in Eqs. (1.3) and (1.5). Szeri [12], and Kokoty and Majumdar [13] showed mathematically that the inertia terms in Eqs. (1.3) and (1.5) become important when $\mathbf{R e}^{*}$ and $\boldsymbol{\Lambda}^{*}$ are on the order of or larger than unity. A novel study on fluid inertia contributions to plain journal bearings was conducted by Reinhart and Lund [14]. They assumed a perturbation expansion of unknowns in Eqs. 1.3 and 1.5 which led to zeroth and first order governing equations. The important short coming of this method was its divergence for large $\mathbf{R e}^{*}$, as mentioned by Grimm [15]. Reinhart and Lund concluded that the added mass coefficient can be significant in applications with short rotors. Mulcahy [16] and Brennen [17] were among the early researchers who explored the effects of fluid inertia on whirling shafts. In more practical terms, Tichy quantified the importance of the fluid inertia forces in squeeze film flows with the analysis of viscoelastic fluids for small centered and off-centered journal whirling motions [5, 6, 7]. The analyses revealed that the viscous and fluid inertia forces are indeed comparable when $\mathbf{R e}^{*}>12$.

Turbulence occurs in applications with high Reynolds numbers. In large Re applications, turbulence effects appear, as indicated by Wilcock [18], as increased power consumption, reduced oil flow and a sharp change in bearing eccentricity. Using "Reynolds velocity decomposition" and taking advantage of the Reynolds stress concept, i.e., $\tau_{i j}^{\prime}=\rho \overline{u_{i}^{\prime} u_{j}^{\prime}}$, turbulence is seen as a direct consequence of the convective inertia terms in the momentum equations [19]. In this methodology, the averaged inertia terms are discarded and the inertia effects are confined to the velocity fluctuations and turbulence [20, 21, 22, 23, 24]. In an effort to develop a turbulent lubrication model consistent with channel flow data, $\mathrm{Ng}$ [20] postulated the application of Reichardt's eddy diffusivity formulation [25]

$$
\frac{\varepsilon_{m}}{\nu}=k\left(y^{+}-\delta^{+} \tanh \frac{y^{+}}{\delta^{+}}\right)
$$


to a shear stress formulation in the turbulent regime of the form

$$
\tau_{i y}=\mu\left(1+\frac{\varepsilon_{m}}{\nu}\right) \frac{\partial u_{i}}{\partial y}, \quad i=x, z
$$

Ng and Pan [21] extended this theory based on a linear flow-pressure model. Elrod and Ng [22], and Safar and Szeri [26] also developed turbulent Reynolds equations with the eddy viscosity assumption, but their flow-pressure relationship was nonlinear. This method, in spite of requiring an iterative scheme, is widely used in literature for different bearing and seal applications [27, 24, 28, 29].

An alternative methodology of including turbulence effects was suggested by Hirs [23. This method does not attempt to analyze turbulence in detail. Instead, it is based on the measured global characteristics of flow, namely the relationship between wall shear stress and average velocity:

$$
\frac{\tau_{w}}{\frac{1}{2} \rho U_{a}^{2}}=n_{o}(\mathbf{R e})^{m_{o}}
$$

where $U_{a}$ is the average film velocity and $\mathbf{R e}=\frac{U_{a} h}{\nu}$ is the Reynolds number based on $U_{a}$. Suganami and Szeri [30] extended turbulence theories to transitional flow regions by introducing a scaling factor. They assumed the range of flow transition in thin film applications to be $400<\mathbf{R e}<900$, based on the local Reynolds number.

The above mentioned studies did not include the inertia terms in the analysis. The temporal inertia terms in Eqs. $1.2-1.5$ are nonlinear products of the velocity components, which result in integrals of the form

$$
I_{i j}=\int_{0}^{h} u_{i} u_{j} d y, \quad i, j=x, z
$$


where $I_{i j}$ are the inertia variables. The early attempts [23, 31, 32, 33, 34, 35] to approximate these nonlinear terms entailed assuming the integral of the velocity product was equal to the product of the integral of the velocities,

$$
\int_{0}^{h} u_{i} u_{j} d y \approx \int_{0}^{h} u_{i} d y \int_{0}^{h} u_{j} d y
$$

However, this assumption is not mathematically correct. To remedy this, Tichy and Bou-Said [3] assumed that the velocity profile across the film thickness in the laminar regime has the form of a superposition of linear (Couette) and parabolic (Poiseuille) flows even when inertia effects are important. In this approach, the inertia effects are limited to the magnitude of the lubricant average velocity. Constantinescu [34, 36], Constantinescu and Galetuse [37, 35], and Frene and Constantinescu [38, 39] extended this model to include both turbulent and laminar regimes [35, 37]. They used Prandtl's mixing length theory and divided the film thickness into near wall, and core regions to find the velocity profile. Their main assumption in this approach was that the convective inertia did not affect the velocity profile, except for its average values, $U_{p}$ and $W_{p}$. Based on the obtained velocity profile, they developed formulations for the integrated convective inertia terms (i.e., inertia variables) and the shear stress difference between the top and bottom of the lubricant film for both laminar and turbulent regimes.

As mentioned before, Elrod and Ng's method requires an iterative process to find the velocity profile, thus, it may encounter convergence issues. Hirs' method has the disadvantage of being dependent on application specific experimental results to predict the shear stress. On the other hand, Constantinescu's method suggests simpler equations for shear and convective inertia terms, which can also be more readily implemented. Bouard, Fillon and Frene [40] compared the three popular 
turbulent models in 1996: the Ng and Pan model, the Elrod and Ng model and the Constantinescu model. They concluded that all of the existing turbulent models gave similar results. They assumed the transition regime to take place between $800<\mathbf{R e}<$ 1500, based on the local Reynolds number. The main common drawback amongst all the aforementioned methods is the exclusion of the temporal inertia terms. Therefore, only the steady state operation of the bearing is addressed and any acceleration or added mass coefficients are ignored. This assumption is often violated for several types of bearings, such as water bearings and especially squeeze film dampers. For these bearings, the journals often whirl in a small orbit around the steady state or equilibrium position. This motion entails centrifugal acceleration, which necessitates the inclusion of temporal inertia in the extended Reynolds equation. In thin film lubrication theory, there is no general agreement of the exact transition region or onset of turbulence. For instance, Taniguchi et al. used $1000<\mathbf{R e}<1500$ as transition region limits. However, $\mathrm{Xu}$ and $\mathrm{Zhu}$ demonstrated in their experiments that the values of the critical Reynolds numbers are functions of the eccentricity [41, 42]. This study adopts 500 and 1000 for these limits.

Water Bearings: Abundance of water, inaccessibility, and oil sealing and supplying difficulties sub-sea, render water-lubricated bearings as one of the most appropriate bearings for underwater applications like submarines. Moreover, they are successfully adopted in ships, turbines, pumps, agricultural machinery [43]. As noted before, these bearings are designed in various geometries and shapes. Rubber pad water bearings are one of the common designs in which the shaft is not fully levitated on a lubricant film and rubber pads accommodate partial contact. Wang et al. conducted an experimental study on flat and concave-stave (pad) water-lubricated bearings used to support a ship propeller shaft [43]. Duan [44] and Liu [45] used the finite element method to analyze rubber bearings. Wang et al. implemented a 3D computational 
fluid dynamics (CFD) model coupled with a fluid structure interaction (FSI) model of water-lubricated rubber bearings [46]. In their study, the influence of axial velocity and rotational speed on the deformation of bearing bush and the distribution of the water film pressure were investigated.

Majumdar et al. studied the effect of grooves on the whirl instability of waterlubricated plain journal bearings with three axial grooves by solving the Reynolds equation numerically [47]. They obtained the dynamic characteristics of the bearing in terms of stiffness and damping coefficients using a first-order perturbation method. However, the results of this study are limited to the laminar regime and inertia effects were not included. In a similar study, Pai and Pai [48] conducted a study on the stability of four and six pad water journal bearings. They solved the traditional Reynolds equation in conjunction with Jakobsson-Floberg-Olsson boundary conditions using a finite difference scheme. Similar to the approach used by Majumdar et al. [47, they ignored the effect of inertia and turbulence in their analysis. Pai et al. used a Computational Fluid Dynamics approach to model fluid flow in a journal bearing with three equal-spaced axial grooves supplied with water from one end of the bearing [49]. They compared their pressure results with experimental data. Cabrera et al. conducted a series of experimental tests on water rubber bearings and concluded that the pressure profile generated in these bearings differs from the rigid ones due to the immense deflection on the rubber surface caused by hydrodynamic pressure [4]. Armentrout et al. [50] in an interesting research effort studied the effect of turbulence and inertia on a single $80^{\circ}$ pad lubricated by water. They compared and contrasted their findings with a corrected turbulence model based on Elrod and Ng's approach [42]. Dousti et al. [51] applied Constantinescue's model with inclusion of temporal inertia effects to short bearing analysis for transient and small perturbation motions. They concluded that in short bearings, convective inertia does not play a major role 
even for high $\mathbf{R e}^{*}$ values.

Squeeze Film Dampers: Since the advent of SFDs, different designs features have been investigated by numerous researchers. Adilleta and Della Pietra in two papers [52, 11] summarized SFD research from 1963 to 2002 in two main categories: characteristics and operating features, and rotordynamics effects. The reader is encouraged to consult these papers for a more complete history and understanding of SFD research. The main difference between a squeeze film damper and a journal bearing is the suppression of the journal's rotation. With the clearance to radius ratio $c / R \sim 10^{-3}$, the Reynolds equation is still the basis for SFD analysis. Early attempts to model the behavior of SFDs focused on using short and long bearing simplifying assumptions to reduce the Reynolds equation and thus, to arrive at analytical solutions [53, 1, 54]. Hahn [53] used Ocvirk (short bearing) and Warner approximations to model the SFD behavior for pressurized $(2 \pi)$ and unpressurized $(\pi)$ films. He applied his model to a rotordynamic analysis of a symmetric shaft supported by roller element bearings and SFDs. Since SFDs provide no hydrodynamic stiffness, the journal trajectory in the housing depends on the vibration levels. Circular centered orbit (CCO) is a common assumption for the journal trajectory inside the housing [55, 56, 57]. El-Shafei and Eranki [56] assumed different trajectories with different radial and circumferential speeds and investigated the validity of the well-known $\pi$-film cavitation theory. Dede et al. [58] studied the damping capacity of sealed SFDs and claimed that the long bearing solution demonstrates a better correlation with experimental results. Similar conclusions were reached by other studies [59, 60, 57]. These aforementioned studies exemplify early research based on the traditional Reynolds equation, which is incapable of predicting added mass effects of SFDs.

Due to the high values of $\mathbf{R e}^{*}$ in the operation of SFDs, inertia effects are experimentally observed in the form of virtual lubricant added mass which mainly affects 
the resultant radial force [61]. The main inertia effect mechanism in SFDs is temporal inertia [11]. However, San Andres investigated the influence of the convective inertia terms in the absence of temporal inertia term(s) in the analysis of a long SFDs [62]. He reported an increase in the radial and tangential forces with the increase of $\mathbf{R e}^{*}$. The nonlinear convective inertia terms due to the negligible surface speed (induced just by non-rotating translatory motions) can be dropped from the analysis [6, 63, 64]. San Andres and Vance [54] developed a linearized perturbation method including the temporal inertia terms but excluding convective inertia for open end SFDs operating within specified small off-centered orbits. They suggested approximate analytical expressions for the linear force coefficients for long and short bearings. Barrett and Gunter also suggested analytical expressions for the force coefficients of SFDs for CCO operation, which is the basis for the ROMAC code SQFDAMP [1]. A similar study was conducted by Szeri et al. for cavitated and uncavitated short SFDs where they suggested closed-form analytical expressions for the linearized force coefficients [65].

Qingchang et al. [66] established a theory to capture the dynamic behavior of finite length open-ended SFDs with shallow feeding grooves. They included temporal inertia and $\mathrm{CCO}$ in their model and compared their results with experimental data. This model exhibited good correlation with the tangential force trend, but large errors were observed in the radial force despite there being an improvement with respect to the traditional model by Zhang et al. [67]. Modest and Tichy [64] studied the effect of fluid film inertia, both convective and temporal, in long SFDs. They concluded that "the lubrication solution for the amplitude of load and pressure can be significantly in error for the high Reynolds number operation of a bearing at low eccentricity ratio. At high eccentricity ratios, however, the lubrication theory can be used with confidence, even at very extreme (high Reynolds number) conditions". 
Since the early 1980's, the Turbomachinery Laboratory at Texas A\&M University has made significant contributions to the area of squeeze film damper research, both theoretically and experimentally [68, 9]. One of San Andres's most significant achievements has been the observation that added mass coefficients are proportional to $c^{-1}$, while the damping coefficients exhibit $c^{-3}$ dependence [9]. Extensive experimental studies have been conducted on open ended and sealed SFDs with grooves at Texas A\&M University's Turbomachinery Laboratory [69, 170, 171, 72, 73, 74, 75, 176, 177, 78]. The comparison of the analytical and experimental data from Ramli et al. [79], shows that a SFD with a shallow groove $(c g / C=3)$ behaves similarly to a single land damper with no groove. Arauz and San Andres [73, 74] studied deeper grooved SFDs with $\mathrm{cg} / C=6$ and 11 experimentally and theoretically by considering circumferential and axial pressure variations in the groove based on short bearing assumptions. The aforementioned studies were primarily limited to SFDs with shallow groove depths. San Andres and Delgado [10] observed large added mass coefficients for SFDs with much deeper grooves. While their experimentally identified damping values correlated well with analytical models [71], large under-predictions were observed in the added mass coefficients. To capture the peculiar behavior exhibited by deep groove SFDs, San Andres suggested an effective nonphysical depth much smaller than the physical depth of the groove to be considered in the analysis [76, 78, 80].

Marmool and Vance suggested a simplified linear finite difference scheme to model supply and discharge holes [81], which was used by Fabiano et al. [82] on the stability analysis of a rotating system. He [83] extended this method to finite SFDs with axial piston ring seals. In this method, each hole is represented as a single rectangular element, which is a rough approximation of the round holes. In addition, for a simple uniform grid, which is often used with the finite difference method, the location of the hole may not be captured accurately. Treatment of holes in this fashion usually 
results in sharp nonphysical peaks in the calculated pressure profile [83]. Chen and Hahn [84] developed a power series solution for hole-fed SFDs with no grooves. In this method, different orders of Reynolds equation are solved depending on the number of terms maintained in the power series solution, which makes the implementation of this method difficult for most practical cases. 


\section{Chapter 2}

\section{Hydrodynamic Bearing Equations}

In this chapter, an extended Reynolds equation is derived governing the behavior of hydrodynamic bearings, including inertia and turbulence effects. The focus of the development is on fixed geometry fluid film journal bearings. In Sec. (2.1), the kinematics of the journal bearing is developed in detail, including the film geometry, velocity and acceleration expressions of an eccentric journal. These expressions are utilized in the interpretation and development of the traditional and extended Reynolds equation. In Sec. 2.2), the traditional Reynolds equation is derived for incompressible fluids. Finally, in Sec. (2.3), the extended Reynolds equation is derived for incompressible fluids which is applicable to the most common industrial fluid film radial bearings.

\subsection{Kinematics of Radial Bearings}

In Sec. 2.3), an extended Reynolds equation is derived for a lubricant film which includes inertia terms with horizontal and vertical velocities and acceleration terms of the lubricant film. In order to support this derivation, it is essential to understand the 
kinematic details of the bearing and establish the grounds for plausible simplifications which may reduce the complexity of the final formulation without neglecting any of the important underlying physics.

In these derivations, two Cartesian coordinate systems and one polar coordinate system, shown in Fig. (2.2), are adopted, however, all final formulations will be developed and presented in terms of displacements in the Cartesian coordinate system and their time derivatives. The $(\hat{X}, \hat{Y})$ Cartesian coordinate is the global reference frame and the $(\xi, \eta)$ Cartesian coordinate system is fixed to the bearing center $O_{b}$. The polar coordinate system is fixed to an arbitrary point on the circumference of the bearing housing, point 1 in Fig. 2.2., with radial coordinate $\hat{n}_{r}$ normal to the bearing (internal) surface pointing towards the center, and the circumferential coordinate $\hat{n}_{\theta}$ tangent to the surface pointing in a counterclockwise direction. Figure 2.1) is a differential segment of the lubricant film around the journal depicted in Fig. (2.2), where horizontal $x$, and vertical $y$ directions correspond to circumferential $n_{\theta}$ and radial $n_{r}$ directions, respectively. The corresponding journal surface point is denoted by point 2 and is collinear with $O_{b}$ and point 1 . The transformation between the polar and Cartesian coordinate systems obeys the following equations

$$
\begin{aligned}
{\left[\begin{array}{l}
\hat{i} \\
\hat{j}
\end{array}\right] } & =\left[\begin{array}{ll}
-\cos \theta & -\sin \theta \\
-\sin \theta & \cos \theta
\end{array}\right]\left[\begin{array}{l}
\hat{n}_{r} \\
\hat{n}_{\theta}
\end{array}\right] \\
{\left[\begin{array}{l}
\hat{n}_{r} \\
\hat{n}_{\theta}
\end{array}\right] } & =\left[\begin{array}{ll}
-\cos \theta & -\sin \theta \\
-\sin \theta & \cos \theta
\end{array}\right]\left[\begin{array}{l}
\hat{i} \\
\hat{j}
\end{array}\right]
\end{aligned}
$$

The following are useful identities obtained from the trigonometric relationships of 


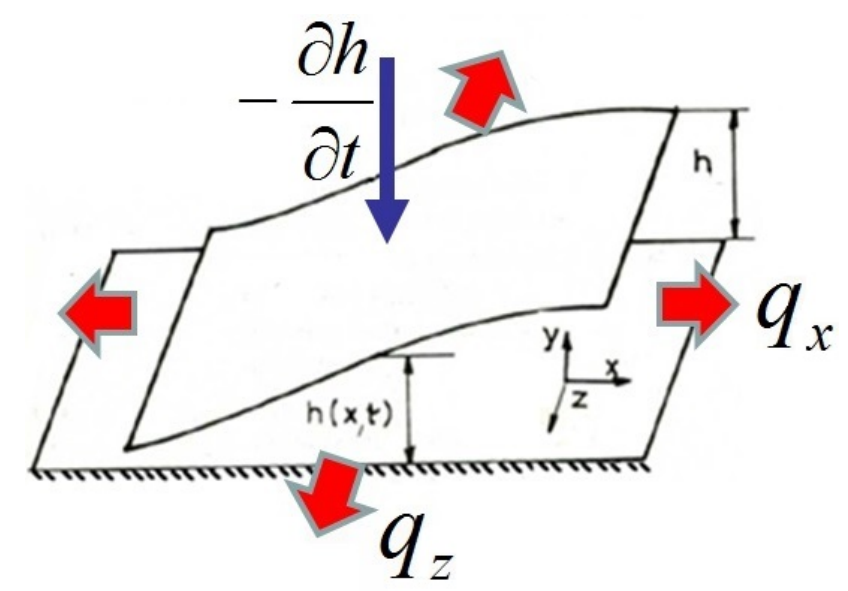

Figure 2.1: Squeeze motion in thin lubricant film, 3]

the triangle $O_{s} 2 O_{b}$ :

$$
\frac{\sin (\alpha)}{\sqrt{X^{2}+Y^{2}}}=\frac{\sin (\theta-\phi)}{R} \quad, \quad \sin (\alpha)=\frac{1}{R}[X \sin (\theta)-Y \cos (\theta)]
$$

These expressions appear numerous times in the kinematic equation development and are therefore important identities. Film thickness along the circumference of the journal and the first and second time derivatives are as follows:

$$
\begin{aligned}
& h=c-X \cos (\theta)-Y \sin (\theta) \\
& \frac{\partial h}{\partial t}=-\dot{X} \cos (\theta)-\dot{Y} \sin (\theta) \\
& \frac{\partial^{2} h}{\partial t^{2}}=-\ddot{X} \cos (\theta)-\ddot{Y} \sin (\theta)
\end{aligned}
$$

Note that as previously mentioned, Fig. (2.1) is the unwrapped representation of the lubricant film of Fig. (2.2). The horizontal coordinate in Fig. (2.1), $x$, corresponds to the circumferential coordinate $\hat{n}_{\theta}$ where we have

$$
d x=(R+c) d \theta=R\left(1+\frac{c}{R}\right) d \theta \approx R d \theta
$$




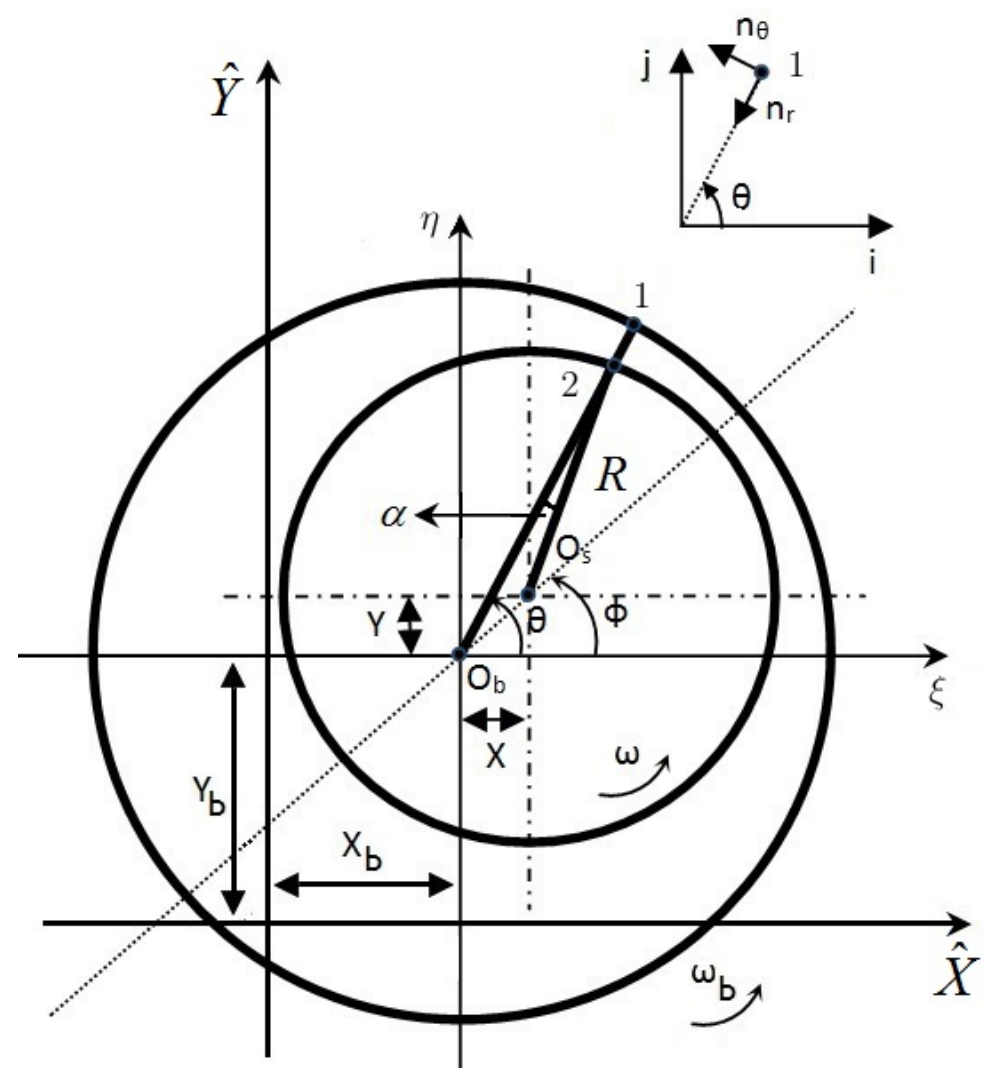

Figure 2.2: Eccentric journal in radial bearing

Since $\frac{c}{R} \approx 10^{-3}$. Therefore, from Eqs. $2.3 \& 2.4$

$$
\sin (\alpha)=\frac{1}{R} \frac{\partial h}{\partial \theta} \approx \frac{1}{(R+c)} \frac{\partial h}{\partial \theta}=\frac{\partial h}{\partial x}
$$

and it can also be seen that $O[\sin (\alpha)]=\frac{c}{R}$ and $O[\cos (\alpha)] \approx 1$.

It should be noted that in this formulation, the most general case is assumed where the bearing and journal experience two types of motions: translatory motion due to vibrations, and rotational motion. The translatory motion of the bearing and journal are expressed by $b$ and $j$ subscripts. Based on Fig. 2.2), we have

$$
X_{j}=X_{b}+X \quad, \quad Y_{j}=Y_{b}+Y
$$


The amplitude of translatory motion is much smaller than the rotational motion amplitude. The bearing and journal centers undergo translational motion only, thus their velocities and accelerations are

$$
\begin{array}{rr}
\overrightarrow{\mathbf{V}}_{o b}=\dot{X}_{b} \hat{i}+\dot{Y}_{b} \hat{j} \quad, \quad \overrightarrow{\mathbf{V}}_{o j}=\dot{X}_{j} \hat{i}+\dot{Y}_{j} \hat{j} \\
\overrightarrow{\mathbf{a}}_{o b}=\ddot{X}_{b} \hat{i}+\ddot{Y}_{b} \hat{j} \quad, \quad \overrightarrow{\mathbf{a}}_{o j}=\ddot{X}_{j} \hat{i}+\ddot{Y}_{j} \hat{j}
\end{array}
$$

The bearing surface velocity (i.e., the general velocity of point " 1 " on the surface of a housing) can be formulated as

$$
\begin{aligned}
\overrightarrow{\mathbf{V}}_{1}= & U_{1} \hat{n}_{\theta}+V_{1} \hat{n}_{r} \\
= & \overrightarrow{\mathbf{V}}_{o b}+(R+c) \omega_{b} \hat{n}_{\theta}=\dot{X}_{b} \hat{i}+\dot{Y}_{b} \hat{j}+(R+c) \omega_{b} \hat{n}_{\theta} \\
= & {\left[-\dot{X}_{b} \cos (\theta)-\dot{Y}_{b} \sin (\theta)\right] \hat{n}_{r} } \\
& \quad+\left[-\dot{X}_{b} \sin (\theta)+\dot{Y}_{b} \cos (\theta)+(R+c) \omega_{b}\right] \hat{n}_{\theta}
\end{aligned}
$$

The circumferential and radial velocity components of the point 1 in Fig. 2.2 can be further broken into translatory and rotational components

$$
\begin{array}{ll}
U_{1}^{t}=-\dot{X}_{b} \sin (\theta)+\dot{Y}_{b} \cos (\theta) \quad, & U_{1}^{r}=(R+c) \omega_{b} \\
V_{1}^{t}=-\dot{X}_{b} \cos (\theta)-\dot{Y}_{b} \sin (\theta) & , \quad V_{1}^{r}=0
\end{array}
$$

In general $O\left[\frac{U_{1}^{t}}{U_{1}^{r}}\right]=\frac{c}{R}$, therefore $U_{1} \approx U_{1}^{r}$. The acceleration of the bearing surface 
is

$$
\begin{aligned}
\overrightarrow{\mathbf{a}}_{1}= & T_{1} \hat{n}_{\theta}+S_{1} \hat{n}_{r} \\
= & \overrightarrow{\mathbf{a}}_{o b}+(R+c) \omega_{b}^{2} \hat{n}_{r}=\ddot{X}_{b} \hat{i}+\ddot{Y}_{b} \hat{j}+(R+c) \omega_{b}^{2} \hat{n}_{r} \\
= & {\left[-\ddot{X}_{b} \sin (\theta)+\ddot{Y}_{b} \cos (\theta)\right] \hat{n}_{\theta} } \\
& \quad+\left[-\ddot{X}_{b} \cos (\theta)-\ddot{Y}_{b} \sin (\theta)+(R+c) \omega_{b}^{2}\right] \hat{n}_{r}
\end{aligned}
$$

The circumferential and radial acceleration components of point 1 in Fig. $(2.2)$ can be broken into their translatory and rotational components

$$
\begin{array}{ll}
T_{1}^{t}=-\ddot{X}_{b} \sin (\theta)+\ddot{Y}_{b} \cos (\theta) \quad, & T_{1}^{r}=0 \\
S_{1}^{t}=-\ddot{X}_{b} \cos (\theta)-\ddot{Y}_{b} \sin (\theta) \quad, \quad & S_{1}^{r}=(R+c) \omega_{b}^{2}
\end{array}
$$

Similar to the development for the bearing surface velocity, the journal surface velocity can be formulated as

$$
\begin{aligned}
\overrightarrow{\mathbf{V}}_{2}= & U_{2} \hat{n}_{\theta}+V_{2} \hat{n}_{r} \\
= & \overrightarrow{\mathbf{V}}_{o j}+R \omega \cos (\alpha) \hat{n}_{\theta}+R \omega \sin (\alpha) \hat{n}_{r} \\
= & {\left[-\dot{X}_{j} \sin (\theta)+\dot{Y}_{j} \cos (\theta)+R \omega \cos (\alpha)\right] \hat{n}_{\theta} } \\
& +\left[-\dot{X}_{j} \cos (\theta)-\dot{Y}_{j} \sin (\theta)+R \omega \sin (\alpha)\right] \hat{n}_{r}
\end{aligned}
$$

By using the identity of Eq. 2.3 and due to the fact that $\alpha$ is small and accordingly $\cos (\alpha) \approx 1$, the circumferential and radial components of the velocity of the journal 
surface, point 2 reduce to

$$
\begin{array}{ll}
U_{2}^{t}=-\dot{X}_{j} \sin (\theta)+\dot{Y}_{j} \cos (\theta) \quad, \quad U_{2}^{r} \approx R \omega \\
V_{2}^{t}=-\dot{X}_{j} \cos (\theta)-\dot{Y}_{j} \sin (\theta) \quad, \quad V_{2}^{r}=\omega[X \sin (\theta)-Y \cos (\theta)]
\end{array}
$$

Similar to point $1, U_{2} \approx U_{2}^{r}$, however translatory and rotational radial velocity of the journal surface are of the same order, i.e., $O\left[V_{2}^{t}\right]=O\left[V_{2}^{r}\right]$. The acceleration of journal surface can be expressed as

$$
\begin{aligned}
\overrightarrow{\mathbf{a}}_{2} & =T_{2} \hat{n}_{\theta}+S_{2} \hat{n}_{r} \\
& =\overrightarrow{\mathbf{a}}_{o j}-R \omega^{2} \sin (\alpha) \hat{n}_{\theta}+R \omega^{2} \cos (\alpha) \hat{n}_{r} \\
& =\left[-\ddot{X}_{j} \sin (\theta)+\ddot{Y}_{j} \cos (\theta)-R \omega^{2} \sin (\alpha)\right] \hat{n}_{\theta} \\
& +\left[-\ddot{X}_{j} \cos (\theta)-\ddot{Y}_{j} \sin (\theta)+R \omega^{2} \cos (\alpha)\right] \hat{n}_{r}
\end{aligned}
$$

The circumferential and radial acceleration components of point 2 in Fig. (2.2) can be broken down further into their translatory and rotational components

$$
\begin{array}{ll}
T_{2}^{t}=-\ddot{X}_{j} \sin (\theta)+\ddot{Y}_{j} \cos (\theta) \quad, \quad T_{2}^{r}=-\omega^{2}[X \sin (\theta)-Y \cos (\theta)] \\
S_{2}^{t}=-\ddot{X}_{j} \cos (\theta)-\ddot{Y}_{j} \sin (\theta) \quad, \quad S_{2}^{r} \approx R \omega^{2}
\end{array}
$$

In the derivation of Reynolds equation, the vertical (radial) velocity difference of the top and bottom of the lubricant film $\left(V_{2}-V_{1}\right)$ appears. By using Eqs. 2.12 2.23), this velocity difference can be expressed in terms of film thickness derivatives and surface velocity as

$$
V_{2}-V_{1}=\frac{\partial h}{\partial t}+\frac{U_{2}^{r}}{R} \frac{\partial h}{\partial \theta} \approx \frac{\partial h}{\partial t}+U_{2}^{r} \frac{\partial h}{\partial x} \approx \frac{\partial h}{\partial t}+U_{2} \frac{\partial h}{\partial x}
$$


Equation (2.24) demonstrates the negligible effect of fluid film curvature in the analysis of radial bearings and therefore, the applicability of the unwrapped film formulation in the analysis of radial bearings as originally assumed by Reynolds.

\subsection{Reynolds Equation For Incompressible Fluids}

In appendix $(\mathrm{B})$, the general Reynolds equation applicable to compressible fluids, Eq. B.42), is developed in full detail as

$$
\begin{gathered}
\frac{1}{6 \mu}\left[\frac{\partial}{\partial x}\left(p h^{3} \frac{\partial p}{\partial x}\right)+\frac{\partial}{\partial z}\left(p h^{3} \frac{\partial p}{\partial z}\right)\right]=2 p\left(V_{2}-V_{1}\right)+2 \frac{\partial p}{\partial t} h \\
+h\left(\frac{\partial}{\partial x}\left[p\left(U_{2}+U_{1}\right)\right]+\frac{\partial}{\partial z}\left[p\left(W_{2}+W_{1}\right)\right]\right) \\
+p\left(U_{1}-U_{2}\right) \frac{\partial h}{\partial x}+p\left(W_{1}-W_{2}\right) \frac{\partial h}{\partial z}
\end{gathered}
$$

In chapter (1), Reynolds' assumptions used to derive his equation for an incompressibe lubricant were listed in detail. Since the fluid is incompressible, its density is invariant with pressure. For this category of fluid, all the equations developed in appendix (B) still apply. However, the continuity equation B.29 simplifies to

$$
\frac{\partial u}{\partial x}+\frac{\partial v}{\partial y}+\frac{\partial w}{\partial z}=0
$$

Integrating Eq. (2.26) across the lubricant film yields

$$
\left(V_{2}-V_{1}\right)+\left(\frac{\partial q_{x}}{\partial x}-\frac{\partial h}{\partial x} U_{2}\right)+\left(\frac{\partial q_{z}}{\partial z}-\frac{\partial h}{\partial z} W_{2}\right)=0
$$

Substituting the unit flow rate expressions from Eqs. B.39 \& B.40 into Eq. 2.27), the most general form of the Reynolds equation for Newtonian incompressible laminar 
inertialess flows can be obtained as

$$
\begin{aligned}
\frac{1}{6 \mu}\left[\frac{\partial}{\partial x}\left(h^{3} \frac{\partial p}{\partial x}\right)\right. & \left.+\frac{\partial}{\partial z}\left(h^{3} \frac{\partial p}{\partial z}\right)\right]=2\left(V_{2}-V_{1}\right) \\
+h & \left(\frac{\partial}{\partial x}\left[U_{2}+U_{1}\right]+\frac{\partial}{\partial z}\left[W_{2}+W_{1}\right]\right) \\
& +\left[U_{1}-U_{2}\right] \frac{\partial h}{\partial x}+\left[W_{1}-W_{2}\right] \frac{\partial h}{\partial z}
\end{aligned}
$$

For radial journal bearings, considering the journal and bearing surface velocities described by Eqs. \begin{tabular}{|l|l|l|l|l|l|l|}
\hline 2.13 & 2.14 & 2.19 & 2.20 & , we have
\end{tabular}

$$
\frac{\partial}{\partial x}\left(U_{2}+U_{1}\right)=\frac{1}{R} \frac{\partial}{\partial \theta}\left(U_{1}^{t}+U_{2}^{t}\right)=\frac{V_{1}^{t}+V_{2}^{t}}{R}
$$

The order of magnitude of this term is $\frac{c}{R}$, which is negligible compared to the other terms in Eq. 2.28). In addition, for a radial journal bearing $W_{1}=W_{2}=0$. And by considering Eq. 2.24), Eq. 2.28 reduces to

$$
\frac{1}{6 \mu}\left[\frac{\partial}{\partial x}\left(h^{3} \frac{\partial p}{\partial x}\right)+\frac{\partial}{\partial z}\left(h^{3} \frac{\partial p}{\partial z}\right)\right]=2 \frac{\partial h}{\partial t}+\left(U_{1}+U_{2}\right) \frac{\partial h}{\partial x}
$$

which is Reynolds equation for incompressible flows. In Eq. 2.30, the main mechanism to produce pressure is viscous shearing, which is represented by $\left(U_{1}+U_{2}\right) \frac{\partial h}{\partial x}$. The squeeze velocity, denoted by $\frac{\partial h}{\partial t}$, is the second mechanism which generates hydrodynamic pressure, and it is the primary damping mechanism for fluid film bearings. 


\subsection{Extended Reynolds Equation For Incompress- ible Fluids}

In section (2.2), Reynolds equation was derived for low Reynolds number applications and laminar regimes by reducing the Navier-Stokes and continuity equations assuming that the inertia terms are negligible. To account for turbulence and inertia effects in high Reynolds number applications, Reynolds equation should be modified. Different approaches adopted by various researchers were reviewed in chapter (1). In this section, the method proposed by Constantinescu is first presented, followed by a more efficient modified approach.

\subsubsection{Constantinescue's method}

For high $\boldsymbol{\Lambda}^{*}$ and $\mathbf{R e}^{*}$, the reduced Navier-Stokes momentum equations with the inclusion of inertia terms are

$$
\begin{aligned}
\rho\left(\frac{\partial u}{\partial t}+u \frac{\partial u}{\partial x}+v \frac{\partial u}{\partial y}+w \frac{\partial u}{\partial z}\right) & =-\frac{\partial p}{\partial x}+\frac{\partial \tau_{x y}}{\partial y} \\
0 & =-\frac{\partial p}{\partial y} \\
\rho\left(\frac{\partial w}{\partial t}+u \frac{\partial w}{\partial x}+v \frac{\partial w}{\partial y}+w \frac{\partial w}{\partial z}\right) & =-\frac{\partial p}{\partial z}+\frac{\partial \tau_{z y}}{\partial y}
\end{aligned}
$$

By using the continuity equation for incompressible fluids (2.26), zero terms can be added to Eqs. 2.31 \& 2.33) to rearrange their convective inertia terms into the following format

$$
\begin{aligned}
& \rho\left(\frac{\partial u}{\partial t}+\frac{\partial u^{2}}{\partial x}+\frac{\partial u v}{\partial y}+\frac{\partial u w}{\partial z}\right)=-\frac{\partial p}{\partial x}+\frac{\partial \tau_{x y}}{\partial y} \\
& \rho\left(\frac{\partial w}{\partial t}+\frac{\partial u w}{\partial x}+\frac{\partial v w}{\partial y}+\frac{\partial w^{2}}{\partial z}\right)=-\frac{\partial p}{\partial z}+\frac{\partial \tau_{z y}}{\partial y}
\end{aligned}
$$


which can then be integrated across the lubricant film by using the Leibniz rule expressed in Eq. (B.35). For radial journal bearing: 1 , we have

$$
\begin{aligned}
& \int_{0}^{h} \frac{\partial u}{\partial t} d y=\frac{\partial q_{x}}{\partial t}+U_{1} V_{1}^{t}-U_{2} V_{2}^{t} \quad, \quad \int_{0}^{h} \frac{\partial w}{\partial t} d y=\frac{\partial q_{z}}{\partial t} \\
& \int_{0}^{h} \frac{\partial u^{2}}{\partial x} d y=\frac{\partial I_{x x}}{\partial x}-U_{2}^{2} \frac{\partial h}{\partial x} \quad, \quad \int_{0}^{h} \frac{\partial u w}{\partial x} d y=\frac{\partial I_{x z}}{\partial x} \\
& \int_{0}^{h} \frac{\partial u v}{\partial y} d y=U_{2} V_{2}-U_{1} V_{1} \quad, \quad \int_{0}^{h} \frac{\partial w v}{\partial y} d y=0 \\
& \int_{0}^{h} \frac{\partial u w}{\partial z} d y=\frac{\partial I_{x z}}{\partial z} \quad, \quad \int_{0}^{h} \frac{\partial w^{2}}{\partial z} d y=\frac{\partial I_{z z}}{\partial z}
\end{aligned}
$$

By substituting Eqs. 2.36-2.39) in the integrated form of Eqs. 2.34 \& 2.35), we have

$$
\begin{aligned}
& \rho I_{x}=\rho\left(\frac{\partial q_{x}}{\partial t}+\frac{\partial I_{x x}}{\partial x}+\frac{\partial I_{x z}}{\partial z}\right)=-h \frac{\partial p}{\partial x}+\tau_{x h}-\tau_{x 0} \\
& \rho I_{z}=\rho\left(\frac{\partial q_{z}}{\partial t}+\frac{\partial I_{x z}}{\partial x}+\frac{\partial I_{z z}}{\partial z}\right)=-h \frac{\partial p}{\partial z}+\tau_{z h}-\tau_{z 0}
\end{aligned}
$$

where

$$
\begin{aligned}
& q_{x}=\int_{0}^{h} u d y \quad, \quad q_{z}=\int_{0}^{h} w d y \\
& I_{x x}=\int_{0}^{h} u^{2} d y \quad, \quad I_{x z}=\int_{0}^{h} u w d y \quad, \quad I_{z z}=\int_{0}^{h} w^{2} d y
\end{aligned}
$$

As was originally assumed by Reynolds, due to the thinness of the lubricant film, its curvature is negligible. Thus, the flow in the film thickness can be approximated by the flow between parallel plates as shown in Fig. (2.3). This flow is the superposition of linear shear or Couette flow and parabolic or Poiseuille flow. The analytical expressions of the flow in the laminar regime in the absence of inertia terms were

\footnotetext{
${ }^{1}$ These results can be also extended to other types of bearings.
} 

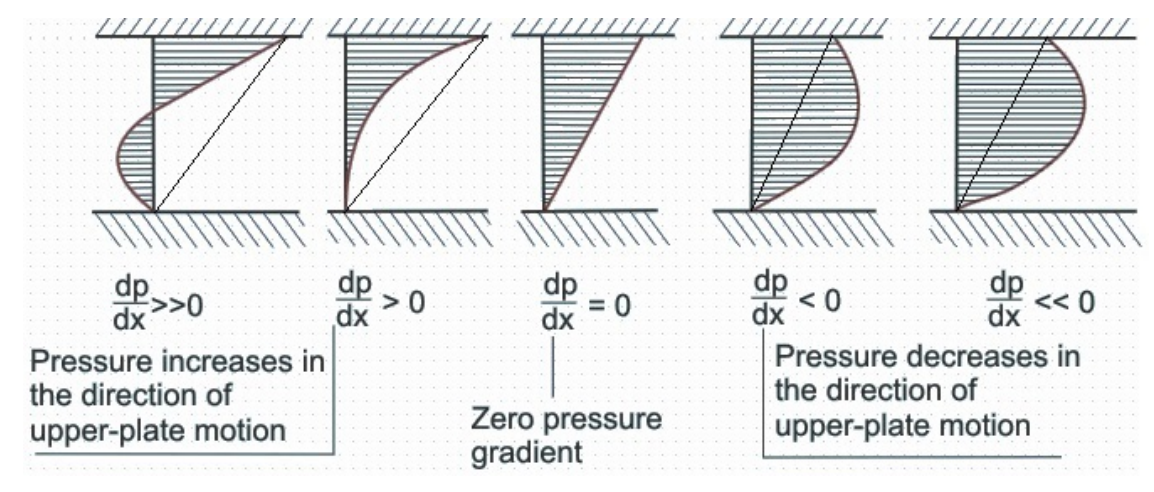

Figure 2.3: Flow Between Plates: a Superposition of Poiseuille and Couette Flows

presented in Eqs. B.27 \& B.28.

For this development, it will be assumed that in the turbulent inertial regime, the flow is also comprised of linear Couette and pressure driven Poiseuille flow components. Thus, in general we have

$$
\begin{aligned}
q_{x} & =U_{p} h+\frac{1}{2}\left(U_{2}+U_{1}\right) h \\
q_{z} & =W_{p} h
\end{aligned}
$$

where $U_{p}$ and $W_{p}$ are the average Poiseuille flow velocities in the $x$ and $z$ directions, which can be obtained explicitly in terms of the pressure gradient in laminar inertialess flow

$$
\begin{aligned}
& U_{p}=-\frac{h^{2}}{12 \mu} \frac{\partial p}{\partial x} \\
& W_{p}=-\frac{h^{2}}{12 \mu} \frac{\partial p}{\partial z}
\end{aligned}
$$

However, in general inertial flows, $U_{p}$ and $W_{p}$ cannot be expressed in terms of the pressure gradient explicitly. In order to deal with this problem, Constantinescu and Galetuse [37] developed a model to include both turbulent and laminar regimes [35, 
37. They used Prandtl's mixing length theory and divided the film thickness into near wall, and core regions to find the velocity profile in both turbulent and laminar regimes for inertialess flows. Then, they extended their model with the main assumption that: the convective inertia does not affect the velocity profile, except for its average values, i.e., $U_{p}$ and $W_{p}$.

Based on the obtained velocity profiles, they suggested formulations for the integrated convective inertia terms, i.e., inertia variables $I_{x x}, I_{x z}, I_{z z}$, and the shear stress difference between the top and the bottom surfaces of the lubricant film in both laminar and turbulent regimes. The resulting equations are

$$
\begin{aligned}
& I_{x x}=\alpha U_{p}^{2} h+\beta\left(U_{1}+U_{2}\right)^{2} h+\gamma U_{p}\left(U_{1}+U_{2}\right) h \\
& I_{x z}=\alpha U_{p} W_{p} h+\frac{1}{2}\left(U_{1}+U_{2}\right) W_{p} h \\
& I_{z z}=\alpha W_{p}^{2} h \\
& \tau_{x h}-\tau_{x 0}=-\frac{k_{x} \mu}{h} U_{p}+\delta \rho\left(\frac{q_{x}}{h}\right)^{2} \frac{\partial h}{\partial x} \\
& \tau_{z h}-\tau_{z 0}=-\frac{k_{z} \mu}{h} W_{p} \\
& \text { laminar : }\left\{\begin{array}{lll}
\alpha=\frac{6}{5}, & \beta=\frac{1}{3}, & \gamma=1 \\
k_{x}=12 & k_{z}=12 & \delta=\frac{2}{15}
\end{array}\right. \\
& \text { Turbulent : } \quad\left\{\begin{array}{l}
\alpha=1, \quad \beta=\left(0.25+\frac{0.885}{\mathbf{R e}^{0.367}}\right), \quad \gamma=1 \\
k_{x}=12+0.0136 \mathbf{R e}^{0.9}, k_{z}=12+0.0043 \mathbf{R} \mathbf{e}^{0.96}, \delta=\frac{1.95^{(2.5}}{\mathbf{R e}^{0.43}}
\end{array}\right.
\end{aligned}
$$

Note that in Eq. (2.54), turbulence is accounted for in the expression of $k_{x}$ and $k_{z}$ based on the local Reynolds number. Based on Constantinescu and Galetuse [37], Eq. (2.51) is corrected by the inclusion of the last term to match experimental data. As mentioned in chapter (1), for this work, the transition regime was chosen to be $500<\mathbf{R e}<1000$ and the coefficients in Eqs. 2.53 \& 2.54) are linearly interpolated 
over this region.

The continuity equation for incompressible fluids, Eq. 2.26), in integrated form can be represented in terms of (unit) flow rates $q_{x}$ and $q_{z}$ as

$$
\frac{\partial q_{x}}{\partial x}+\frac{\partial q_{z}}{\partial z}+\frac{\partial h}{\partial t}=0
$$

Substituting Eqs. (2.51 \& 2.52) into Eqs. 2.40 \& 2.41) results in

$$
\begin{aligned}
& U_{p}=-\frac{h^{2}}{k_{x} \mu} \frac{\partial p}{\partial x}-\frac{\rho h}{k_{x} \mu}\left(I_{x}-\delta\left(\frac{q_{x}}{h}\right)^{2} \frac{\partial h}{\partial x}\right) \\
& W_{p}=-\frac{h^{2}}{k_{z} \mu} \frac{\partial p}{\partial z}-\frac{\rho h}{k_{z} \mu} I_{z}
\end{aligned}
$$

and the flow rates in Eqs. 2.44 \& 2.45) can be obtained by replacing $U_{p}$ and $W_{p}$ from Eqs. 2.57\& 2.56) as

$$
\begin{aligned}
q_{x} & =-\frac{h^{3}}{k_{x} \mu} \frac{\partial p}{\partial x}+\frac{U_{2}+U_{1}}{2} h-\frac{\rho h^{2}}{k_{x} \mu} I_{x}+\delta \frac{\rho q_{x}^{2}}{k_{x} \mu} \frac{\partial h}{\partial x} \\
q_{z} & =-\frac{h^{3}}{k_{z} \mu} \frac{\partial p}{\partial z}-\frac{\rho h^{2}}{k_{z} \mu} I_{z}
\end{aligned}
$$

It should be noted that Eqs. 2.58 \& 2.59) contain inertia terms that are dependent on flow rates, hence, an iterative scheme is required for this calculation (see Sec. 4.2). For a radial journal bearing, the extended Reynolds equation is obtained by substituting Eqs. 2.58 \& 2.59) into Eq. 2.55) and eliminating negligible terms based on Eq. 2.29), and finally rearranging the terms into the following form

$$
\frac{\partial}{\partial x}\left(\frac{h^{3}}{k_{x} \mu} \frac{\partial p}{\partial x}\right)+\frac{\partial}{\partial z}\left(\frac{h^{3}}{k_{z} \mu} \frac{\partial p}{\partial z}\right)=\frac{\partial h}{\partial t}+\frac{U_{2}+U_{1}}{2} \frac{\partial h}{\partial x}+I_{t}+I_{c}+C
$$


where $C$ is the correction term given by

$$
C=\frac{\delta \rho}{k_{x} \mu} q_{x}^{2} \frac{\partial^{2} h}{\partial x^{2}}+\frac{2 \delta \rho}{k_{x} \mu} q_{x} \frac{\partial q_{x}}{\partial x} \frac{\partial h}{\partial x}
$$

while $I_{t}$ and $I_{c}$ are the temporal and convective inertia terms expressed by

$$
\begin{aligned}
I_{t}=-\frac{\rho}{\mu} & {\left[\frac{h^{2}}{k_{x}} \frac{\partial^{2} q_{x}}{\partial x \partial t}+\frac{h^{2}}{k_{z}} \frac{\partial^{2} q_{z}}{\partial z \partial t}+\frac{1}{k_{x}} \frac{\partial h^{2}}{\partial x} \frac{\partial q_{x}}{\partial t}\right] } \\
I_{c}=-\frac{\rho}{\mu} & {\left[\frac{1}{k_{x}} \frac{\partial h^{2}}{\partial x} \frac{\partial I_{x x}}{\partial x}+\frac{1}{k_{x}} \frac{\partial h^{2}}{\partial x} \frac{\partial I_{x z}}{\partial z}\right.} \\
& \left.+\frac{h^{2}}{k_{x}} \frac{\partial^{2} I_{x x}}{\partial x^{2}}+h^{2} \frac{\partial^{2} I_{x z}}{\partial x \partial z}\left(\frac{1}{k_{x}}+\frac{1}{k_{z}}\right)+\frac{h^{2}}{k_{z}} \frac{\partial^{2} I_{z z}}{\partial z^{2}}\right]
\end{aligned}
$$

where based on Eqs.(2.48 2.50), the spatial derivatives of the inertia variables are

$$
\begin{aligned}
& \frac{\partial I_{x x}}{\partial x}=\frac{I_{x x}}{h} \frac{\partial h}{\partial x}+2 \alpha U_{p} \frac{\partial U_{p}}{\partial x} h+\gamma\left(U_{1}+U_{2}\right) \frac{\partial U_{p}}{\partial x} h \\
& \frac{\partial^{2} I_{x x}}{\partial x^{2}}=\frac{\partial I_{x x}}{\partial x} \frac{1}{h} \frac{\partial h}{\partial x}+\frac{I_{x x}}{h} \frac{\partial^{2} h}{\partial x^{2}}-\frac{I_{x x}}{h^{2}}\left(\frac{\partial h}{\partial x}\right)^{2}+2 \alpha\left(\frac{\partial U_{p}}{\partial x}\right)^{2} h \\
&+2 \alpha U_{p} \frac{\partial^{2} U_{p}}{\partial x^{2}} h+\left[2 \alpha U_{p}+\gamma\left(U_{1}+U_{2}\right)\right] \frac{\partial U_{p}}{\partial x} \frac{\partial h}{\partial x}+\gamma\left(U_{1}+U_{2}\right) \frac{\partial^{2} U_{p}}{\partial x^{2}} h \\
& \frac{\partial I_{x z}}{\partial x}=\frac{I_{x z}}{h} \frac{\partial h}{\partial x}+\alpha \frac{\partial U_{p}}{\partial x} W_{p} h+\alpha U_{p} \frac{\partial W_{p}}{\partial x} h+\frac{1}{2}\left(U_{1}+U_{2}\right) \frac{\partial W_{p}}{\partial x} h \\
& \frac{\partial I_{x z}}{\partial z}=\alpha \frac{\partial U_{p}}{\partial z} W_{p} h+\alpha U_{p} \frac{\partial W_{p}}{\partial z} h+\frac{1}{2}\left(U_{1}+U_{2}\right) \frac{\partial W_{p}}{\partial z} h \\
& \frac{\partial I_{x z}}{\partial x \partial z}=\frac{\partial I_{x z}}{\partial z} \frac{1}{h} \frac{\partial h}{\partial x}+\alpha \frac{\partial^{2} U_{p}}{\partial x \partial z} W_{p} h+\alpha \frac{\partial U_{p}}{\partial z} \frac{\partial W_{p}}{\partial x} h \\
&+\alpha U_{p} \frac{\partial^{2} W_{p}}{\partial x \partial z} h+\alpha \frac{\partial U_{p}}{\partial x} \frac{\partial W_{p}}{\partial z} h+\frac{1}{2}\left(U_{1}+U_{2}\right) \frac{\partial^{2} W_{p}}{\partial x \partial z} h \\
& \frac{\partial I_{z z}}{\partial z}=2 \alpha W_{p} \frac{\partial W_{p}}{\partial z} h \\
& \frac{\partial^{2} I_{z z}}{\partial z^{2}}=2 \alpha\left(\frac{\partial W_{p}}{\partial z}\right)^{2} h+2 \alpha W_{p} \frac{\partial^{2} W_{p}}{\partial z^{2}} h
\end{aligned}
$$

In this study, the first two terms in Eq. (2.62) are referred to as the "primary" temporal inertia, and the last term as the "secondary" temporal inertia term. Temporal 
inertia terms and the approach to model them are elaborated on in Sect. 4.1.3). The derived extended Reynolds equation (2.60) in the absence of inertia and correction terms and for bearings operating in the laminar regime collapses to the classical Reynolds equation (2.30).

\subsubsection{The Modified Approach}

As mentioned in Sec. (2.3), implementation of Constatinescu's method requires an iterative scheme to capture the pressure dependent average velocities $U_{p}$ and $W_{p}$. The pressure profile that emerges numerically from this method is not an "analytic" or "differentiable" function at the edge(s) of the cavitation region. This results in a step in the $U_{p}$ and $W_{p}$ profiles calculated based on the $x$ derivative of the pressure profile as it appears in Eqs. 2.56 \& 2.57). The unrealistic and erroneous steps (see

Fig. (4.7) ) often grow exponentially as iterations advance, and result in divergence of the algorithm. Therefore, in this section a modified version of Constantinescu's mehod is presented, which eliminates the issues associated with Constantinescu's approach and leads to a more robust solution algorithm.

In this approach, (convective) inertia variables are modified by eliminating the spatial derivatives of the average velocities, $U_{p}$ and $W_{p}$, in the circumferential direction 
such that Eqs. 2.64 2.70 become

$$
\begin{aligned}
\frac{\partial I_{x x}}{\partial x} & =\frac{I_{x x}}{h} \frac{\partial h}{\partial x} \\
\frac{\partial^{2} I_{x x}}{\partial x^{2}} & =\frac{\partial I_{x x}}{\partial x} \frac{1}{h} \frac{\partial h}{\partial x}+\frac{I_{x x}}{h} \frac{\partial^{2} h}{\partial x^{2}}-\frac{I_{x x}}{h^{2}}\left(\frac{\partial h}{\partial x}\right)^{2} \\
\frac{\partial I_{x z}}{\partial x} & =\frac{I_{x z}}{h} \frac{\partial h}{\partial x} \\
\frac{\partial I_{x z}}{\partial z} & =\alpha \frac{\partial U_{p}}{\partial z} W_{p} h+\alpha U_{p} \frac{\partial W_{p}}{\partial z} h+\frac{1}{2}\left(U_{1}+U_{2}\right) \frac{\partial W_{p}}{\partial z} h \\
\frac{\partial^{2} I_{x z}}{\partial x \partial z} & =\frac{\partial I_{x z}}{\partial z} \frac{1}{h} \frac{\partial h}{\partial x} \\
\frac{\partial I_{z z}}{\partial z} & =2 \alpha W_{p} \frac{\partial W_{p}}{\partial z} h \\
\frac{\partial^{2} I_{z z}}{\partial z^{2}} & =2 \alpha\left(\frac{\partial W_{p}}{\partial z}\right)^{2} h+2 \alpha W_{p} \frac{\partial^{2} W_{p}}{\partial z^{2}} h
\end{aligned}
$$

In addition, the turbulence model suggested by Constantinescu in Eq. 2.54) is slightly modified as

$$
k_{x}=12+0.0113 \mathbf{R} \mathbf{e}^{0.9}, k_{z}=12+0.0036 \mathbf{R e}^{0.96}
$$

such that, it results in a better correlation with CFD data presented in Sec. 4.3.1. 


\section{Chapter 3}

\section{Solution Method}

The (extended) Reynolds equation 2.60 developed in the previous chapter is a 2D quasiharmonic equation that in general does not have any general analytical solution. Historically, simplifying assumptions and other methods have been adopted to make some analytical solutions of this equation feasible. Dousti et al. [51] reduced the extended Reynolds equation by using the short bearing assumption and thereby obtained an analytical solution. However, with the advancement and expansion of computing power, analytical solutions are no longer a necessity. Numerical approaches can now be implemented to provide practical solutions with the combination of a practical computation time and high accuracy level. The Finite Element (FE) method has been proven to an effective numerical solution method and is utilized in this work to solve the extended Reynolds equation, Eq. 2.60).

\subsection{Galerkin Method}

Generally, fluid mechanics problems are described by nonconservative differential equations and associated boundary conditions, which makes the use of the direct 


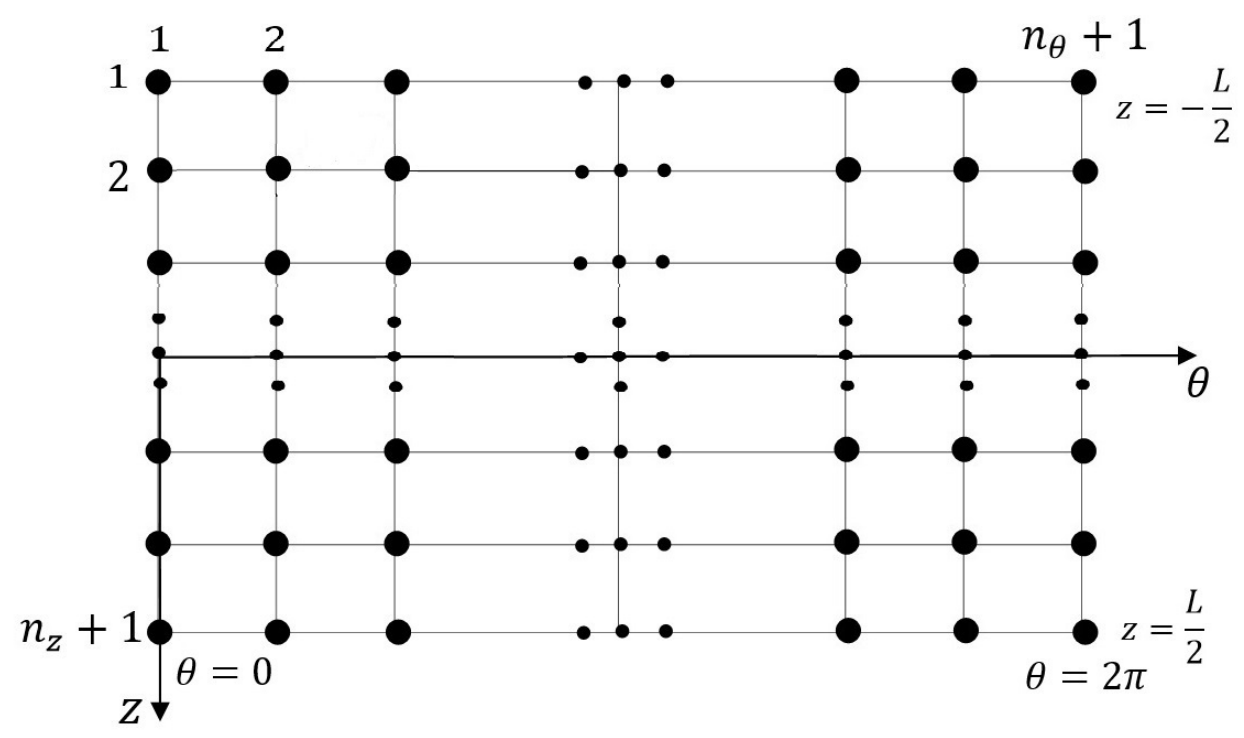

Figure 3.1: Pressure domain and boundaries

FE methods such as virtual work or the variational approach impossible. However, the FE formulations for problems of this type may still be implemented by using weighted residual methods, of which the Gakrkin method is the most widely used.

For a sleeve bearing or squeeze film damper, the pressure region is a $2 \mathrm{D}$ domain extended $2 \pi R$ in the $x$ (or $2 \pi$ in the $n_{\theta}$ ) direction, and $L$ in the $z$ axial direction, shown in Fig. (3.1). Due to the circular geometry of sleeve bearings, their FE model is periodic in the $x$ direction (i.e., the first and the last nodes in this direction coincide with each other). The (traditional and/or extended) Reynolds equation 2.60 and/or 2.30 can be expressed in the following form

$$
\vec{\nabla}^{T}\left(h^{3} \vec{\nabla}_{G} p\right)+f(x, z)=0
$$


where $\vec{\nabla}$ and $\vec{\nabla}_{G}$ are the vector derivative operators defined as

$$
\begin{aligned}
& \left.\vec{\nabla}=\frac{\partial}{\partial x} \hat{\mathbf{i}}+\frac{\partial}{\partial z} \hat{\mathbf{k}}=\left\{\begin{array}{c}
\frac{\partial}{\partial x} \\
\frac{\partial}{\partial z}
\end{array}\right\} \begin{array}{c}
G_{x} \frac{\partial}{\partial x} \\
G_{z} \frac{\partial}{\partial z}
\end{array}\right\}
\end{aligned}
$$

Axial end boundary conditions for Eq. (3.1) can be expressed in two general forms. For sleeve bearings and open end squeeze film dampers, the pressure at the axial ends is constant and equal to the ambient pressure

$$
p=p_{o} \quad @ \quad z= \pm \frac{L}{2}
$$

For sealed squeeze film dampers, gradient boundary conditions of the following general form apply

$$
\pm \frac{\partial p}{\partial z}=H(\theta)\left(p-p_{o}\right) \quad @ \quad z= \pm \frac{L}{2}
$$

As mentioned before, an exact solution for a pressure profile which satisfies Eq. (3.1) in the entire pressure domain Fig. (3.1) does not exist; however, the Galerkin method provides a weak form solution that converges to the exact solution. In this formulation, the following approximate solution based on nodal values of pressure can be used

$$
\tilde{p}(x, z)=\mathbf{N}^{T} \vec{P}=\left[\begin{array}{llll}
N_{1} & N_{2} & \cdots & N_{D}
\end{array}\right]\left\{\begin{array}{c}
P_{1} \\
P_{2} \\
\vdots \\
P_{D}
\end{array}\right\}
$$

where $\tilde{p}$ is the approximate solution, $\{P\}$ is the vector of nodal pressure values, and 
$N_{i}, 1 \leqslant i \leqslant D$ are the shape functions. This solution satisfies the Reynolds equation in integral vectorial form as

$$
\iint_{A} \mathbf{N}\left[\vec{\nabla}^{T}\left(h^{3} \vec{\nabla}_{G} \tilde{p}\right)+f(x, z)\right] d x d z=\left\{\begin{array}{c}
0 \\
0 \\
\vdots \\
0
\end{array}\right\}
$$

which can be rewritten in scalar form as

$$
\iint_{A} N_{i}\left[\vec{\nabla}^{T}\left(h^{3} \vec{\nabla}_{G} \tilde{p}\right)+f(x, z)\right] d x d z=0, \quad 1 \leqslant i \leqslant D
$$

where $A$ is the integration pressure domain in Fig. (3.1). Utilizing the technique of integration by parts, the first term in Eq. (3.8) can be calculated as

$$
N_{i} \vec{\nabla}^{T}\left(h^{3} \vec{\nabla}_{G} \tilde{p}\right)=\vec{\nabla}^{T}\left[N_{i}\left(h^{3} \vec{\nabla}_{G} \tilde{p}\right)\right]-\left(\vec{\nabla} N_{i}\right)^{T}\left(h^{3} \vec{\nabla}_{G} \tilde{p}\right)
$$

The 2D integration of the first term in the right hand side of Eq. (3.9) over the pressure domain in Fig. (3.1) is transformed by using the divergence theorem as follows

$$
\iint_{A} \vec{\nabla}^{T}\left[N_{i}\left(h^{3} \vec{\nabla}_{G} \tilde{p}\right)\right] d x d z=\int_{S} N_{i}\left(h^{3} \vec{\nabla}_{G} \tilde{p}\right) \cdot \hat{\mathbf{n}} d s
$$

where $\hat{\mathbf{n}}$ is the outward unit normal vector on the boundary $S$. The normal vectors on $z=\frac{L}{2}$ and $z=-\frac{L}{2}$ are $\hat{\mathbf{k}}$ and $-\hat{\mathbf{k}}$, respectively. Note that the contribution of Eq. (3.10) is restricted to the equations related to the nodes on the boundaries. By 
substituting Eqs. 3.6 and 3.9 into Eq. (3.8), we have

$$
\left[\iint_{A}\left(\vec{\nabla} N_{i}\right)_{1 \times 2}^{T}\left(h^{3} \vec{\nabla}_{G} \mathbf{N}^{T}\right)_{2 \times D} d x d z\right]_{1 \times D} \vec{P}_{D \times 1}=\left[\iint_{A} N_{i} f(x, z) d x d z\right]_{1 \times 1}
$$

where $1 \leq i \leq D$. The system of equations formed in matrix notation can be written as

$$
\begin{gathered}
\mathbf{K} \vec{P}=\overrightarrow{\mathbf{F}} \\
\mathbf{K}=\left[\iint_{A}\left(\vec{\nabla} \mathbf{N}^{T}\right)_{D \times 2}^{T}\left(h^{3} \vec{\nabla}_{G} \mathbf{N}^{T}\right)_{2 \times D} d x d z\right]_{D \times D} \\
\overrightarrow{\mathbf{F}}=\left[\iint_{A} \mathbf{N} f(x, z) d x d z\right]_{D \times 1}
\end{gathered}
$$

where $\mathbf{K}$ and $\overrightarrow{\mathbf{F}}$ are the fluidity matrix and load vector, respectively. The fluidity matrix and load vector are amended by taking into account the boundary condition effects. There are various algorithms available to solve Eq. (3.12). The most common method is to use LU decomposition 1 to decompose the fluidity matrix $\mathbf{K}$ into the product of lower, $\mathbf{L}$, and upper, $\mathbf{U}$, triangular matrices. Then, the inverse of the $\mathbf{L}$ matrix can be multiplied by $\overrightarrow{\mathbf{F}}$ followed by back substitution to solve for the pressure values of each node. To account for the cavitation phenomenon, each node with a negative pressure value is truncated to zero before being used in the back substitution process.

\footnotetext{
${ }^{1}$ In numerical analysis, LU decomposition (where 'LU' stands for 'Lower Upper', and also called LU factorization) factors a matrix as the product of a lower triangular matrix and an upper triangular matrix. The product sometimes includes a permutation matrix as well. The LU decomposition can be viewed as the matrix form of Gaussian elimination. Computers usually solve square systems of linear equations using the LU decomposition, and it is also a key step when inverting a matrix, or computing the determinant of a matrix. The LU decomposition was introduced by mathematician Alan Turing, see 85.
} 


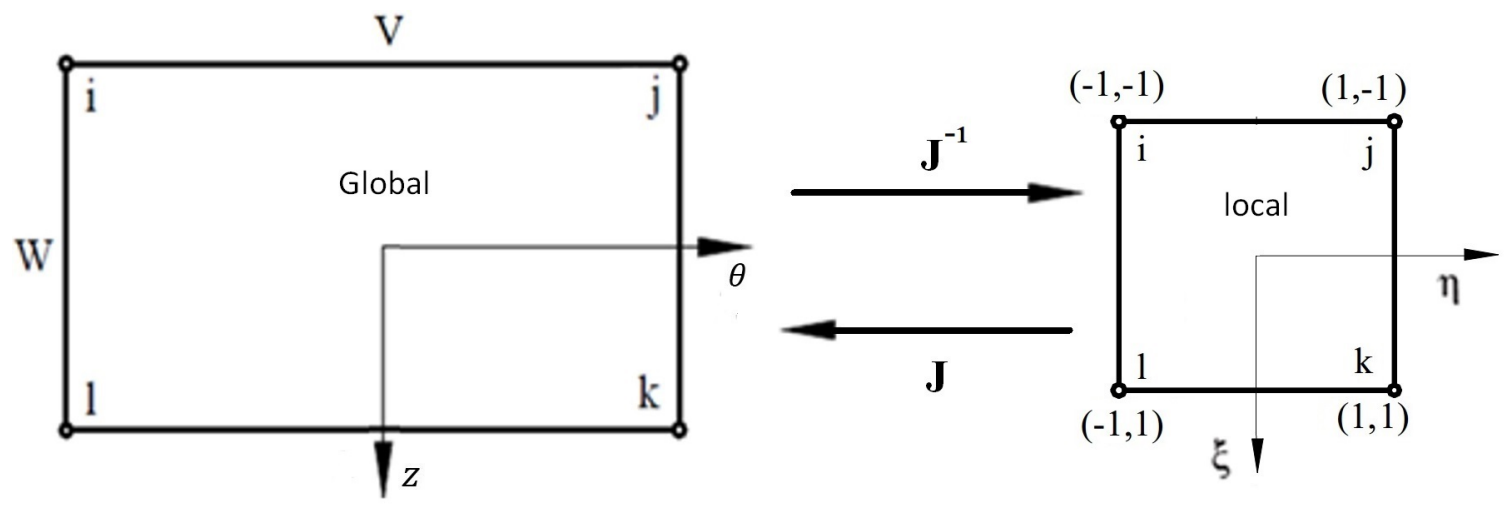

Figure 3.2: Global and local isoprametric elements

\subsection{Rectangular Isoparametric Element}

As previously mentioned and depicted in Fig. (3.1), the unwrapped fluid film forms a rectangular pressure domain which is most convenient to map with rectangular isoparametric elements shown in Fig. (3.2).

Isoparametric elements carry the property that their shape functions are used to interpolate both element geometry and field variables (pressure, film thickness, etc.). That is, the pressure of a point within an element can be expressed in terms of nodal d.o.f and shape functions, $N_{i}$, which are expressed in local coordinates. Similarly the global position (coordinates) of a point within the element can be expressed in terms of the global nodal positions and the same shape functions, $N_{i}$, which are also functions of the local coordinates

$$
\begin{aligned}
& N_{i, j, k, l}=\frac{1}{4}(1 \pm \eta)(1 \pm \xi) \\
& \mathbf{N}_{e}(\eta, \xi)=\left[\begin{array}{llll}
N_{i} & N_{j} & N_{k} & N_{l}
\end{array}\right]^{T}
\end{aligned}
$$


The global location of an arbitrary point in an element is expressed as

$$
\left\{\begin{array}{c}
\theta \\
z
\end{array}\right\}=\left[\begin{array}{cccc}
\theta_{i} & \theta_{j} & \theta_{k} & \theta_{l} \\
z_{i} & z_{j} & z_{k} & z_{l}
\end{array}\right] \mathbf{N}_{e}(\eta, \xi)
$$

and similarly the field variables are denoted as

$$
\phi=\mathbf{N}_{e}^{T}\left\{\begin{array}{c}
\phi_{i} \\
\phi_{j} \\
\phi_{k} \\
\phi_{l}
\end{array}\right\}=\mathbf{N}_{e}^{T} \overrightarrow{\boldsymbol{\Phi}}_{e}
$$

Equation 3.17) can be used to transform the global differentials into local ones and vice versa.

$$
\left\{\begin{array}{c}
d \theta \\
d z
\end{array}\right\}=\left[\begin{array}{ll}
\frac{\partial \theta}{\partial \eta} & \frac{\partial \theta}{\partial \xi} \\
\frac{\partial z}{\partial \eta} & \frac{\partial z}{\partial \xi}
\end{array}\right]\left\{\begin{array}{c}
d \eta \\
d \xi
\end{array}\right\}=\mathbf{J}^{T}\left\{\begin{array}{c}
d \eta \\
d \xi
\end{array}\right\}
$$

where $\mathbf{J}$ is the Jacobian matrix

$$
\mathbf{J}=\left[\begin{array}{cc}
\frac{\partial \theta}{\partial \eta} & \frac{\partial z}{\partial \eta} \\
\frac{\partial \theta}{\partial \xi} & \frac{\partial z}{\partial \xi}
\end{array}\right]=\left[\begin{array}{c}
\frac{\partial \mathbf{N}^{T}}{\partial \eta} \\
\frac{\partial \mathbf{N}^{T}}{\partial \xi}
\end{array}\right]_{2 \times 4}\left[\begin{array}{cc}
\theta_{i} & z_{i} \\
\theta_{j} & z_{j} \\
\theta_{k} & z_{k} \\
\theta_{l} & z_{l}
\end{array}\right]_{4 \times 2}
$$

To calculate the element fluidity matrix $\mathbf{K}_{e}$ and the element force vector $\overrightarrow{\mathbf{F}}_{e}$, Equations $3.13 \& 3.14$ are computed, and since the shape functions are described in local coordinates, the derivative operators are transformed to local coordinates as well. To 
this end, the Jacobian matrix is also utilized

$$
\begin{aligned}
\vec{\nabla}_{e} & =\frac{\partial}{\partial \eta} \hat{\mathbf{i}}+\frac{\partial}{\partial \xi} \hat{\mathbf{k}}=\mathbf{J} \vec{\nabla} \\
\vec{\nabla}_{G} & =\mathbf{J}_{G}^{-1} \vec{\nabla}_{e}
\end{aligned}
$$

where

$$
\begin{gathered}
\mathbf{J}_{G}^{-1}=\left[\begin{array}{cc}
G_{x} \mathbf{J}_{11}^{-1} & G_{x} \mathbf{J}_{12}^{-1} \\
G_{z} \mathbf{J}_{21}^{-1} & G_{z} \mathbf{J}_{22}^{-1}
\end{array}\right] \\
d x d z=R d \theta d z=R \operatorname{det}(\mathbf{J}) d \eta d \xi
\end{gathered}
$$

Thus,

$$
\begin{gathered}
\mathbf{K}_{e}=\left[\iint_{A_{e}}\left(\mathbf{N}_{e}^{T} \overrightarrow{\mathbf{h}}_{e}\right)^{3}\left(\mathbf{J}^{-1} \vec{\nabla}_{e} \mathbf{N}_{e}^{T}\right)_{4 \times 2}^{T}\left(\mathbf{J}_{G}^{-1} \vec{\nabla}_{e} \mathbf{N}_{e}^{T}\right)_{2 \times 4} R \operatorname{det}(\mathbf{J}) d \eta d \xi\right]_{4 \times 4} \\
\overrightarrow{\mathbf{F}}_{e}=\left[\iint_{A_{e}} \mathbf{N}_{e} f(x, z) R \operatorname{det}(\mathbf{J}) d \eta d \xi\right]_{4 \times 1}
\end{gathered}
$$

Calculation of the integrals in Eqs. 3.25 \& 3.26) can be computationally intensive [86], especially considering the fact that it needs be calculated for every element. Therefore, Gaussian quadrature is used to approximate the 2D integrations as

$$
\int_{-1}^{1} \int_{-1}^{1} f(x, y) d x d y=\sum_{i=1}^{2} \sum_{j=1}^{2} \mathbf{W}_{i} \mathbf{W}_{j} f\left(x_{i}, y_{j}\right), x_{i}= \pm \frac{\sqrt{3}}{3}, y_{i}= \pm \frac{\sqrt{3}}{3}
$$


and thus the elemental fluidity matrix and load vector can be calculated as

$$
\begin{gathered}
\mathbf{K}_{e}=\frac{k_{x} h^{3} W}{6 V}\left[\begin{array}{cccc}
2 & -2 & -1 & 1 \\
-2 & 2 & 1 & -1 \\
-1 & 1 & 2 & -2 \\
1 & -1 & -2 & 2
\end{array}\right]+\frac{k_{z} h^{3} V}{6 W}\left[\begin{array}{cccc}
2 & 1 & -1 & -2 \\
1 & 2 & -2 & -1 \\
-1 & -2 & 2 & 1 \\
-2 & -1 & 1 & 2
\end{array}\right] \\
\overrightarrow{\mathbf{F}}_{e}=\frac{f V W}{4}\left[\begin{array}{c}
1 \\
1 \\
1 \\
1
\end{array}\right]
\end{gathered}
$$

and assembled into a global fluidity matrix and load vector. As shown in Fig. (3.1), for a grid with $n_{z}$ and $n_{\theta}$ elements in axial and circumferential directions, respectively, $D=\left(n_{\theta}+1\right)\left(n_{z}+1\right)$ and the fluidity matrix is a $D \times D$ square matrix and the load vector is $D$ dimensional. 


\section{Chapter 4}

\section{Fixed Geometry Sleeve Bearings}

Sleeve bearings, illustrated in Fig. 4.1), are the most basic fluid film journal bearings. The geometry of these bearings remain unchanged for various operating conditions. In these bearings, there often exists one or more axial grooves used for lubrication supply purposes. However, sometimes, especially in low viscosity lubricant bearings like water bearings, no groove is present, i.e., a full cylindrical housing, which requires satisfaction of periodicity in the circumferential direction as a boundary condition. The current ROMAC bearing codes: THBRG, THPAD, and MAXBRG are not presently capable of handling this geometry and boundary condition. In addition, the underlying theory for these codes is inadequate for modeling low viscosity lubricants.

In Sec. (4.1), the perturbation method is applied to the extended Reynolds equation derived in chapter (2), resulting in zero order and auxiliary equations. These equations are utilized to find the steady state equilibrium position and linear rotordynamic coefficients of the bearing. In Sec. 4.2, the structure of SLEEVEBRG, an isothermal hydrodynamic code to analyze fixed geometry sleeve bearings is presented in detail. Section (4.3) discusses the results obtained from SLEEVEBRG for three cases: i) a single water lubricated pad ii) a single axial groove fixed geometry oil 


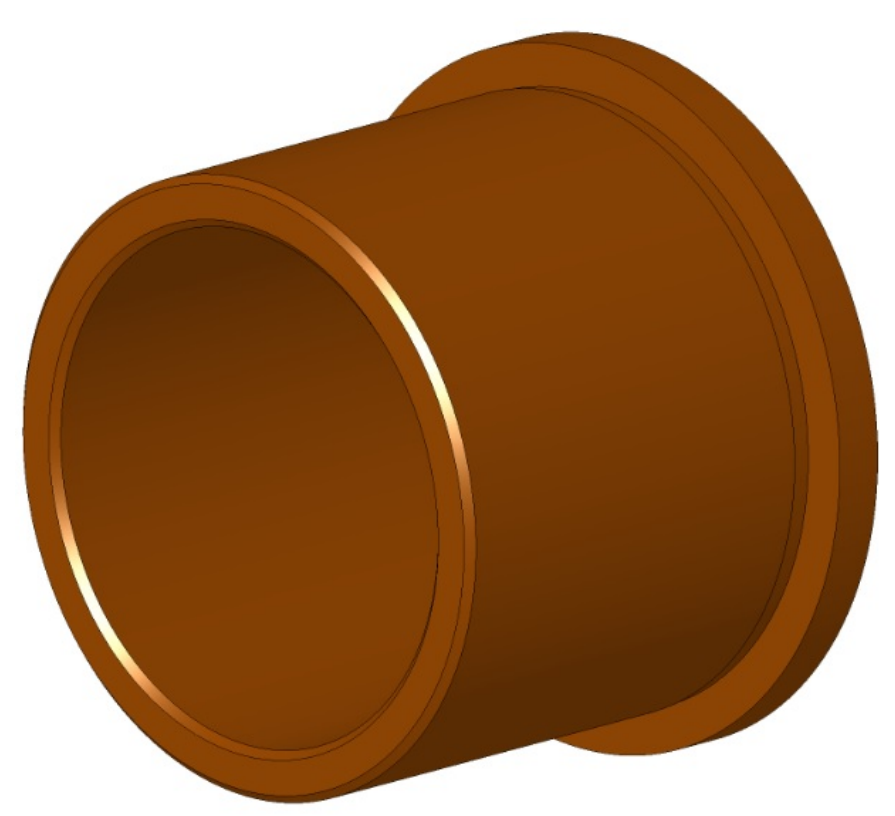

Figure 4.1: sleeve bearing with no axial groove

bearing, and iii) a full cylindrical water bearing .

\subsection{Methodology and Governing Equations}

Due to the journal rotation, journal bearings operate at an equilibrium position with a steady state condition. External dynamic forces perturb the journal from this equilibrium position. It is a common practice to identify the stiffness, damping and (in this study) added mass properties of the bearing for small perturbations around the equilibrium position and use them to evaluate the rotordynamic performance of the bearing. To this end, the extended Reynolds equation (2.60) developed in Sec. (2.3) and reprinted here for convenience, is used

$$
\frac{\partial}{\partial x}\left(\frac{h^{3}}{k_{x} \mu} \frac{\partial p}{\partial x}\right)+\frac{\partial}{\partial z}\left(\frac{h^{3}}{k_{z} \mu} \frac{\partial p}{\partial z}\right)=\frac{\partial h}{\partial t}+\frac{U_{2}+U_{1}}{2} \frac{\partial h}{\partial x}+I_{t}+I_{c}+C
$$


In general, two methods can be applied to calculate the bearing dynamic coefficients: numerical and analytical. In the first approach, the journal is "numerically" perturbed a small amount about its equilibrium position and the pressure difference is used to calculate the rotordynamic coefficients $(K, C$, and $M)$. In the second method, the Reynolds equation is perturbed "analytically" and "auxiliary" equations governing the pressure derivatives are derived. These pressure derivatives are used to calculate the dynamic coefficients [87, 88]. In this work, the analytical method is used. And in this section, the derivations of the auxiliary equations are presented. Position perturbation, $\Delta \zeta$, results in the film thickness variation. Similarly, velocity and acceleration perturbations yield perturbations of the time derivatives of the film thickness. The variation of film thickness and its time derivatives, consequently, perturbs the pressure. Using the variable $\zeta$ to represent the horizontal and/or the vertical displacements $(X, Y)$, shown in Fig. 2.2 results in

$$
\begin{aligned}
& h=h_{o}+\Delta h=h_{o}+h_{, \zeta} \Delta \zeta \\
& \frac{\partial h}{\partial t}=\frac{\partial h_{o}}{\partial t}+\Delta \frac{\partial h}{\partial t}=\frac{\partial h_{o}}{\partial t}+\left(\frac{\partial h}{\partial t}\right)_{, \dot{\zeta}} \Delta \dot{\zeta} \\
& \frac{\partial^{2} h}{\partial t^{2}}=\frac{\partial^{2} h_{o}}{\partial t^{2}}+\Delta \frac{\partial^{2} h}{\partial t^{2}}=\frac{\partial^{2} h_{o}}{\partial t^{2}}+\left(\frac{\partial^{2} h}{\partial t^{2}}\right)_{, \ddot{\zeta}} \Delta \ddot{\zeta} \\
& p=p_{o}+p_{, \zeta} \Delta \zeta+p_{, \dot{\zeta}} \Delta \dot{\zeta}+p_{, \ddot{\zeta}} \Delta \ddot{\zeta}
\end{aligned}
$$

where the perturbation derivative is defined as $A_{, \zeta}=\frac{\partial A}{\partial \zeta}$, which is mathematically equivalent to partial derivatives. By considering equations $2.4-2.6)$, we have

$$
\begin{array}{ll}
\zeta=x: \quad h_{, \zeta}=\left(\frac{\partial h}{\partial t}\right)_{, \dot{\zeta}}=\left(\frac{\partial^{2} h}{\partial t^{2}}\right)_{, \ddot{\zeta}}=-\cos (\theta) \\
\zeta=y: \quad h_{, \zeta}=\left(\frac{\partial h}{\partial t}\right)_{, \dot{\zeta}}=\left(\frac{\partial^{2} h}{\partial t^{2}}\right)_{, \ddot{\zeta}}=-\sin (\theta)
\end{array}
$$


To derive the auxiliary Reynolds equations, the nonlinear terms in the extended Reynolds equation Eq. 2.60) are expanded by the Maclaurin series method around the equilibrium position using Eqs. 4.2 4.5), and only the zeroth and the first order terms are preserved. To obtain the auxiliary equations resulting in the stiffness coefficients, the journal position is perturbed. The full derivation of this process is explained in detail in appendix $(\mathrm{A})$.

\subsubsection{Zeroth Order Equation}

The zeroth order Reynolds equation is

$$
\frac{\partial}{\partial x}\left(\frac{h_{o}^{3}}{k_{x} \mu} \frac{\partial p_{o}}{\partial x}\right)+\frac{\partial}{\partial z}\left(\frac{h_{o}^{3}}{k_{z} \mu} \frac{\partial p_{o}}{\partial z}\right)=\frac{U_{1}+U_{2}}{2} h_{o}+I_{c o}+C_{o}
$$

Note that in general, $U_{1}=0$, since the bearing surface is stationary. The subscript "o" represents the zeroth order variable. The solution of this equation determines the load carrying pressure profile, $p_{o}$. And from $p_{o}$, the resultant forces in the $x$ and $y$ directions can be calculated

$$
\begin{aligned}
F_{X} & =-\iint p_{o} \cos \theta d x d z \\
F_{Y} & =-\iint p_{o} \sin \theta d x d z
\end{aligned}
$$

or in a concise form

$$
F_{\zeta}=\iint p_{o} \frac{\partial h}{\partial \zeta} d x d z
$$

Equations $4.9 \& 4.10$ are generally either solved directly by imposing a fixed displacement, i.e., equilibrium position, to find the load carrying capacity of the bearings, or inversely by finding the equilibrium position for a given externally applied steady 
state load.

\subsubsection{Auxiliary Equation, Stiffness and Damping}

The first order auxiliary Reynolds equation due to the displacement perturbation is

$$
\begin{array}{r}
\frac{\partial}{\partial x}\left\{\frac{h_{o}^{3}}{k_{x} \mu} \frac{\partial}{\partial x}\left(p_{, \zeta}\right)\right\}+\frac{\partial}{\partial z}\left\{\frac{h_{o}^{3}}{k_{z} \mu} \frac{\partial}{\partial z}\left(p_{, \zeta}\right)\right\}=\frac{U_{1}+U_{2}}{2} \frac{\partial h_{, \zeta}}{\partial x}+I_{c, \zeta} \\
+C_{, \zeta}-\frac{\partial}{\partial x}\left\{\frac{3 h_{o}^{2}}{k_{x} \mu} \frac{\partial p_{o}}{\partial x} h_{, \zeta}\right\}-\frac{\partial}{\partial z}\left\{\frac{3 h_{o}^{2}}{k_{z} \mu} \frac{\partial p_{o}}{\partial z} h_{, \zeta}\right\}
\end{array}
$$

Note that by regarding the iterative nature of the extended Reynolds equation derived here, the perturbations of the convective inertia, $I_{c}$, and the correction terms, $C$, are assumed to be small such that $U_{p}$ and $W_{p}$ do not change significantly. Therefore, they are considered invariant and equal to the values calculated at the equilibrium position. The direct and cross-coupled stiffness coefficients are calculated by integrating the perturbed pressure solution

$$
K_{\varrho \zeta}=-F_{\varrho, \zeta}=-\iint p_{, \zeta} \frac{\partial h}{\partial \varrho} d x d z
$$

where $\varrho, \zeta$ are either $X$ or $Y$. The cavitation region is assumed invariant under small perturbations. Thus, if cavitation is predicted for the steady state solution of a particular node, i.e., $P_{o}$, the perturbed cavitation solution of that node is also set to zero.

The circumferential surface speed contributes to the steady state flow rates. The radial squeeze velocity, on the other hand, results in the squeeze phenomenon and the resulting temporal derivatives of the flow rates. This mechanism generates the damping property of the bearing. The auxiliary Reynolds equation corresponding to velocity perturbation is obtained by perturbing the squeeze term in Eq. 4.1, since 
this term is the only term dependent on the journal center velocities. Therefore,

$$
\frac{\partial}{\partial x}\left(\frac{h_{o}^{3}}{k_{x} \mu} \frac{\partial p_{, \dot{\zeta}}}{\partial x}\right)+\frac{\partial}{\partial z}\left(\frac{h_{o}^{3}}{k_{z} \mu} \frac{\partial p_{, \dot{\zeta}}}{\partial z}\right)=\left(\frac{\partial h}{\partial t}\right)_{, \dot{\zeta}}
$$

and similarly, the solution of the above equation yields damping coefficients as

$$
C_{\varrho \zeta}=-F_{\varrho, \dot{\zeta}}=-\iint p_{, \dot{\zeta}} \frac{\partial h}{\partial \varrho} d x d z
$$

\subsubsection{Auxiliary Equation, Temporal Inertia and Added Mass}

The inclusion of the temporal inertia terms in Eq. 2.60) (i.e., $I_{t}$ ) enables the calculation of the added mass coefficients. Tichy and Bou-Said [3] and Dousti et al. [51] suggested formulations for temporal inertia based on the short bearing assumption. San Andres and Delgado [89] obtained the temporal inertia formulation for finite length SFDs in the laminar regime, but neglected the "secondary" temporal inertia term in Eq. (2.62). In this study, the temporal inertia effects are captured in full and presented in the most general form.

In perturbation analysis assuming small motions, $I_{t}$ appears in the order of perturbation motions. Figure (4.2) and Figure (2.1) depict the squeeze motion in elemental and macro scale, respectively. In Eq. 2.62, reprinted here as Eq. (4.16) for convenience, the three terms can be seen to be implicitly dependent on the squeeze acceleration, $\frac{\partial^{2} h}{\partial t^{2}}$,

$$
I_{t}=-\frac{\rho}{\mu}\left[\frac{h^{2}}{k_{x}} \frac{\partial^{2} q_{x}}{\partial x \partial t}+\frac{h^{2}}{k_{z}} \frac{\partial^{2} q_{z}}{\partial z \partial t}+\frac{1}{k_{x}} \frac{\partial h^{2}}{\partial x} \frac{\partial q_{x}}{\partial t}\right]
$$

Note that in the above equation, the coefficients $k_{x}$ and $k_{z}$ are both equal to 12 unless turbulence is present, in which case Eq. (2.78) applies. In order to express the 


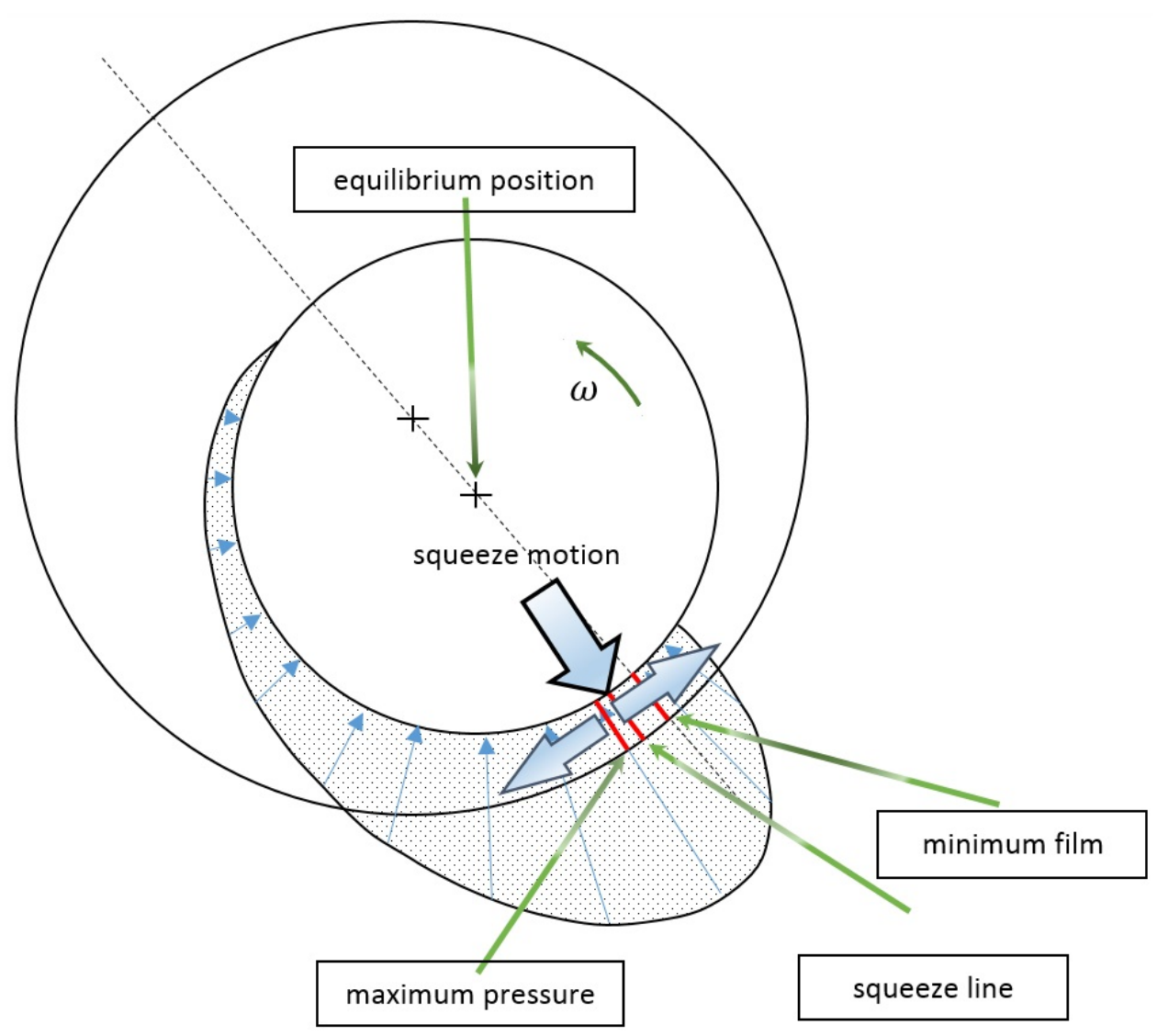

Figure 4.2: squeeze motion

axial and circumferential acceleration in terms of the radial squeeze acceleration, the time derivative of the integrated continuity equation 2.55 is utilized

$$
\frac{\partial^{2} q_{x}}{\partial x \partial t}+\frac{\partial^{2} q_{z}}{\partial z \partial t}+\frac{\partial^{2} h}{\partial t^{2}}=0
$$

Eq. (4.17) contains two unknowns, i.e., the time derivative of the flow rate gradients in $x$ and $z$ directions. In other words, the vertical acceleration results directly in variation of the flow accelerations in the $x$ and $z$ directions, but not directly in the flow accelerations. Note that the flow acceleration is pressure driven. This pressure, generated by the acceleration as indicated in Eq. (4.5), is a perturbation pressure with respect to the steady state pressure, so it is appropriate to assume that the 
pressure profile is not significantly affected by the acceleration perturbation. Using this concept (and in the absence of a suitable second equation to solve for the two unknowns), the squeeze acceleration is assumed to be distributed in the $x$ and $z$ directions proportionally to the local steady state pressure gradients as

$$
\begin{aligned}
& \frac{\partial^{2} q_{x}}{\partial x \partial t}=-Q_{x} \frac{\partial^{2} h}{\partial t^{2}} \quad, \quad Q_{x}=\frac{\left|\frac{\partial p_{o}}{\partial x}\right|}{\left|\frac{\partial p_{o}}{\partial x}\right|+\left|\frac{\partial p_{o}}{\partial z}\right|} \\
& \frac{\partial^{2} q_{z}}{\partial z \partial t}=-Q_{z} \frac{\partial^{2} h}{\partial t^{2}} \quad, \quad Q_{z}=\frac{\left|\frac{\partial p_{o}}{\partial z}\right|}{\left|\frac{\partial p_{o}}{\partial x}\right|+\left|\frac{\partial p_{o}}{\partial z}\right|}
\end{aligned}
$$

It should be remembered that Eqs. (4.18 \& 4.19) are used to link the local surface squeeze acceleration to the gradients of the squeeze acceleration regardless of what the flow accelerations, $\frac{\partial q_{x}}{\partial t}$ and $\frac{\partial q_{z}}{\partial t}$ are at that location.

In the cavitated region, where pressure and its spatial gradients vanish and Eqs. $4.18 \& 4.19$ are not valid, two possible scenarios may occur. Either it can be assumed that the lubricant film is ruptured and no acceleration takes place such that

$$
Q_{x}=Q_{z}=0
$$

or a fully flooded lubricating condition exists and a continuous film even in the cavitated region can be assumed. For high ambient water lubricated bearings, this scenario makes better sense. Hence, in the absence of a known hydrodynamic pressure, the simplest approach might be to assume equal distribution of the squeeze acceleration in the $x$ and $z$ directions as

$$
Q_{x}=Q_{z}=\frac{1}{2}
$$


The above two equations $4.20 \& 4.21$ will yield different results, and should therefore be chosen appropriately for a given application.

The next step in calculating the temporal inertia is to extract the $\frac{\partial q_{x}}{\partial t}$ term which appeared in the "secondary" temporal inertia in Eq. 4.16). This term appears due to the eccentricity of journal in the bearing which causes film variation in the circumferential direction and increases with journal eccentricity. In order to calculate $\frac{\partial q_{x}}{\partial t}$, Eq. 4.18 can be integrated numerically in the circumferential direction. This calculation requires a circumferential location for which $\frac{\partial q_{x}}{\partial t}$ is zero. In the lack of any experimental or numerical data on the subject, in this study, this point is selected to coincide with the maximum pressure location, namely $x_{p m a x}$. This assumption suggests that due to the squeeze acceleration, flow squirts and accelerates to the sides of the maximum pressure location shown in Fig. (4.2) as if an imaginary wall separates the two regions. And it should be noted that $x_{\text {pmax }}$ in general is different from the minimum film thickness angle.

$$
\frac{\partial q_{x}}{\partial t}=-\int_{x_{\text {pmax }}}^{x} Q_{x} \frac{\partial^{2} h}{\partial t^{2}} d x
$$

By perturbing the journal position acceleration terms, the auxiliary Reynolds equations leading to the lubricant added mass coefficients is obtained as

$$
\begin{aligned}
& \frac{\partial}{\partial x}\left(\frac{h_{o}^{3}}{k_{x} \rho} \frac{\partial p_{, \ddot{\zeta}}}{\partial x}\right)+\frac{\partial}{\partial z}\left(\frac{h_{o}^{3}}{k_{z} \rho} \frac{\partial p_{, \ddot{\zeta}}}{\partial z}\right)= \\
& \quad \frac{1}{k_{x}} \frac{\partial h_{o}^{2}}{\partial x} \int_{x_{p \max }}^{x} Q_{x}\left(\frac{\partial^{2} h}{\partial t^{2}}\right)_{\ddot{,}} d x+Q_{x} \frac{h_{o}^{2}}{k_{x}}\left(\frac{\partial^{2} h}{\partial t^{2}}\right)_{, \ddot{\zeta}}+Q_{z} \frac{h_{o}^{2}}{k_{z}}\left(\frac{\partial^{2} h}{\partial t^{2}}\right)_{\ddot{,}}
\end{aligned}
$$

where $Q_{x}, Q_{z}$ are calculated based on the solution of the zeroth order Reynolds equation. Note that Eq. 4.23) is not explicitly dependent on the lubricant viscosity, but only on its density. Similar to stiffness and damping, the solution of Eq. (4.23) is 
used to find the lubricant added mass coefficients

$$
M_{\varrho \zeta}=-\frac{\partial F_{\varrho}}{\partial \ddot{\zeta}}=-\iint \frac{\partial p}{\partial \ddot{\zeta}} \frac{\partial h}{\partial \varrho} d x d z
$$

\subsubsection{Rigid Rotor Stability}

The derivations developed in Sec. 4.1) result in rotordynamic coefficients of the bearing $(K, C, M)$. In turn, these parameters can be used in a simple rigid rotor stability test to judge the overall performance of the bearing. The free vibration of the two degree of freedom journal in the bearing can be expressed by

$$
\mathrm{MQ̈}+\mathrm{C} \dot{\mathrm{Q}}+\mathrm{KQ}=0
$$

where the coefficient matrices $\mathbf{M}, \mathbf{C}, \mathbf{K}$ are the mass, damping, and stiffness matrices calculated from the shaft mass and the auxiliary equations developed in Secs. 4.1.3 \&4.1.2, such that

$$
\begin{aligned}
\mathbf{K} & =\left(\begin{array}{cc}
K_{X X} & K_{X Y} \\
K_{Y X} & K_{Y Y}
\end{array}\right) \\
\mathbf{C} & =\left(\begin{array}{cc}
C_{X X} & C_{X Y} \\
C_{Y X} & C_{Y Y}
\end{array}\right) \\
\mathbf{M} & =\left(\begin{array}{cc}
M_{\text {shaft }}+M_{X X} & M_{X Y} \\
M_{Y X} & M_{\text {shaft }}+M_{Y Y}
\end{array}\right)
\end{aligned}
$$

The associated eigenvalue problem can be solved to determine the eigenvalues of the equation of motion. The rigid rotor dynamical system of Eq. 4.25 has four complex 
conjugate eigenvalues of the following form

$$
\lambda_{1,2}=\alpha_{1} \pm j \beta_{1} \quad, \quad \lambda_{3,4}=\alpha_{2} \pm j \beta_{2} \quad, \quad \alpha_{1} \geq \alpha_{2}
$$

The stability of the system is determined by $\alpha_{1}$, the real part of the eigenvalue with the greater real part. Defining

$$
\delta=\frac{-2 \pi \alpha_{1}}{\beta_{1}}
$$

then if $\delta$ is positive, the system is stable; and if it is negative or zero, it is unstable.

\subsection{SLEEVEBRG}

A computer code named SLEEVEBRG has been developed to analyze fixed geometry bearings, also known as sleeve bearings. An isothermal/isoviscous assumption is made in the formulation of this code making it most suitable for the analysis of water and low viscosity bearings. This code is based on the extended Reynolds equation and therefore, takes into account all the associated hydrodynamic effects elaborated on in Sect. (2.3).

The water bearing for which SLEEVEBRG is well suited is a relatively long full cylinder with no axial grooves as shown in Fig. (4.3). This type of bearing design is primarily used in subsea applications, where the bearing is enclosed in a high pressure water jacket which feeds the lubricating fluid (i.e., water) into the clearance. The ambient pressure in these bearings depends on the depth of the water, and can, therefore, be very high, which can result in the cavitation phenomenon being partially or totally suppressed. Since there is no axial groove, i.e., a full cylindrical inner surface, the $2 \pi$ periodicity needs to be enforced in the circumferential direction. The viscosity of water is an order of magnitude lower than the typical oil-based 


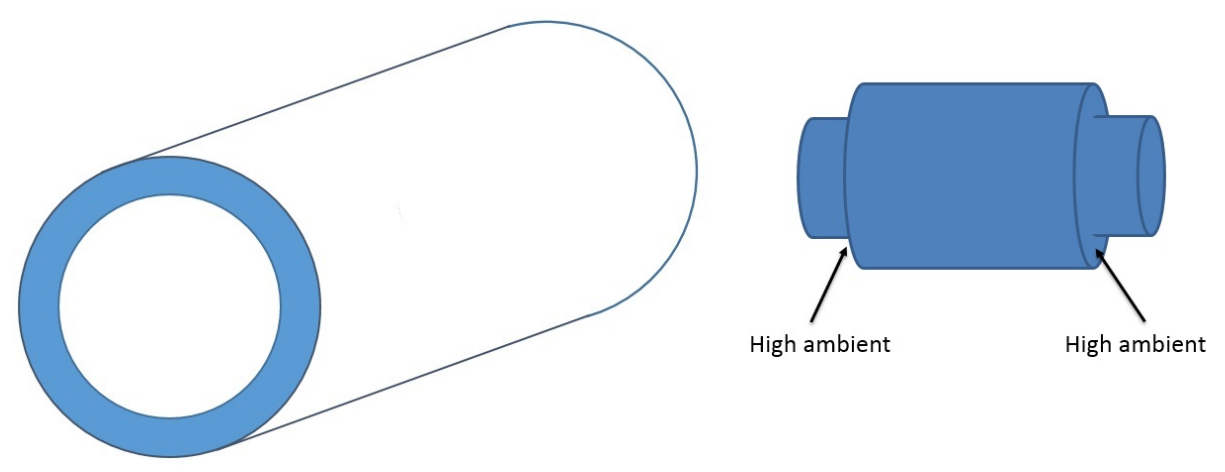

Figure 4.3: Cylindrical water bearing

lubricant. Therefore, the associated heat generation is not a significant concern in water bearings, and thus, can be ignored, justifying the omission of the thermal analysis in this code. On the other hand, high Reynolds numbers occur in these applications which necessitates the inclusion of inertia and turbulence effects in the analysis.

SLEEVEBRG can only be applied to a limitedly range of common oil lubricated sleeve bearings. In standard oil lubricated sleeve bearings, a number of axial grooves are included for lubrication purposes. Axial grooves can be seen as pressure relaxation locations, which isolate the lobes and breaks the $2 \pi$ periodicity boundary condition. Since the $2 \pi$ periodic condition is not satisfied in other ROMAC codes, i.e., THBRG or MAXBRG, their use for cylindrical sleeve bearings with no axial grooves can result in significant errors. The applicability of SLEEVEBRG to oil bearings is limited to single axial groove bearings in which the groove is located in the cavitated region and operate with low rotational speeds and/or load loads, where the thermal effects are not significant.

The main structure of SLEEVEBRG is illustrated in Fig. (4.4). SLEEVEBRG follows an iterative scheme, the goal of which is to converge to steady-state nodal pressure values for the entire grid space. Initially, for a fixed journal position, the 


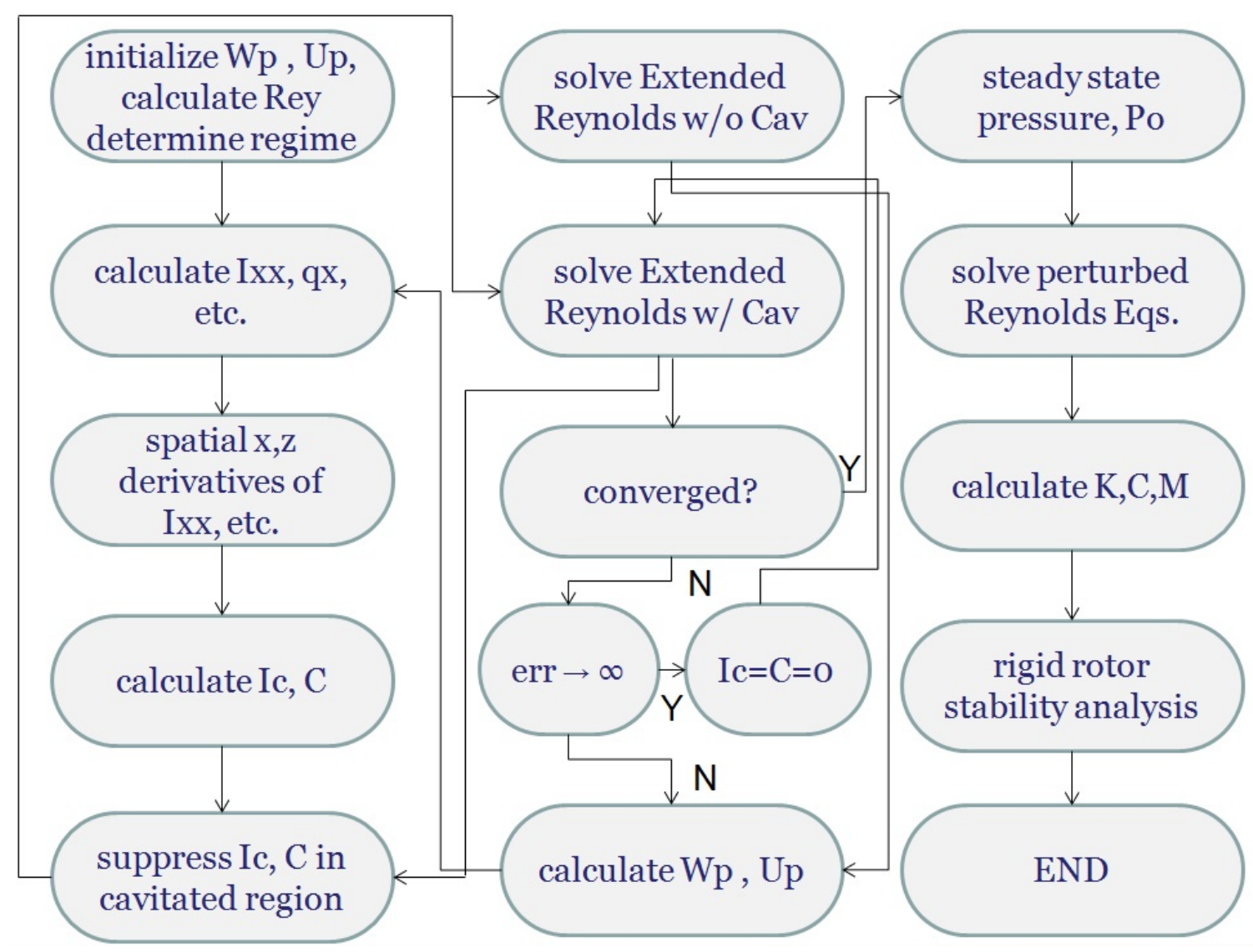

Figure 4.4: Structure of computer code SLEEVEBRG

local Reynolds number array is calculated based on the shaft surface speed, and the traditional Reynolds equation (with no inertia effects) is solved for the pressures. These values are then used to calculate $U_{p}$ and $W_{p}$ based on Eqs. 2.46 \& 2.47), which in turn are utilized to calculate the convective inertia Eqs. $2.482 .49 \& 2.50$ and flow rates Eqs. $2.44 \& 2.45$. Based on these values, the global load vector, $\overrightarrow{\mathbf{F}}$ in Eq. 3.12 is calculated. The new values of $U_{p}$ and $W_{p}$ are calculated using Eqs. 2.56 \& 2.57), so that the convective inertia effects are included. Note that the fluidity matrix, $\mathbf{K}$, is independent from the inertia effects, thus, once calculated, it does not need to be updated. The zeroth order Reynolds equation, Eq. 4.8), is used to solve for $p_{o}$. A convergence criterion based on the root mean square (rms) error 
of successive values of $p_{o}$ is used to test for convergence of the pressure field solution. The error in each calculation step is defined as

$$
\operatorname{err}=\frac{\sqrt{\sum_{i=1}^{D}\left(p_{o i}^{j+1}-p_{o i}^{j}\right)^{2}}}{D P_{u}} \quad, \quad j=1,2,3, \ldots
$$

where $j$ is the iteration number, and error is normalized by the nominal $P_{u}$ defined as

$$
P_{u}=\frac{F_{a}}{2 L R}
$$

It should be noted that the goal of the iterative procedure is to include convective inertia effects; however, SLEEVEBRG is designed to remove these effects in case of divergence, and in this case only the turbulence effects are taken into account in the solution algorithm.

A few subtle remarks are also worth mentioning. FEA solutions entail a backsubstitution procedure. To account for cavitation, any negative nodal pressure value is set to zero. However, in reality the pressure profile smoothly transitions (i.e., $\left.\frac{\partial p}{\partial \theta}=0\right)$ at the cavitation circumferential boundaries, where $p=0$, as well. Backsubstitution does not explicitly enforce the derivative boundary condition, however it has been shown to predict accurate pressure profiles when compared with experimental data [42]. As described in detail in chapter (2), a number of derivatives are involved in Eqs. (2.56-2.63) in order to calculate the convective inertia and correction terms. This process can yield unrealistic jumps (or steps) in $I_{c}$ and $C$ profiles at the cavitation boundary, where $p=0$ occurs. This fictitious step in many cases grows unboundedly and results in the algorithm's divergence, see Fig. (4.5). To remedy this issue the modified spatial derivative expressions of the inertia variables expressed in Eqs. 2.71-2.77) are used in place of Eqs. 2.64 2.70. 


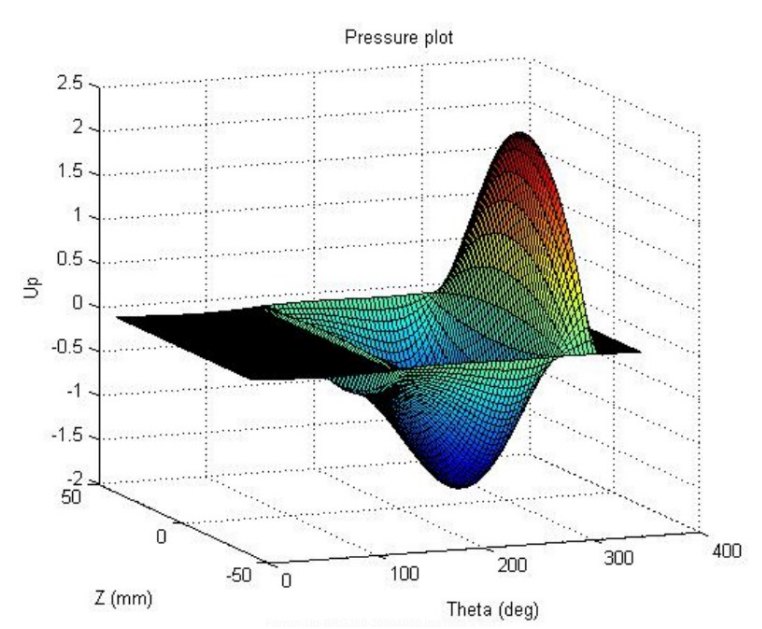

(a) $U_{p}$ profile in $x$ and $z$ directions

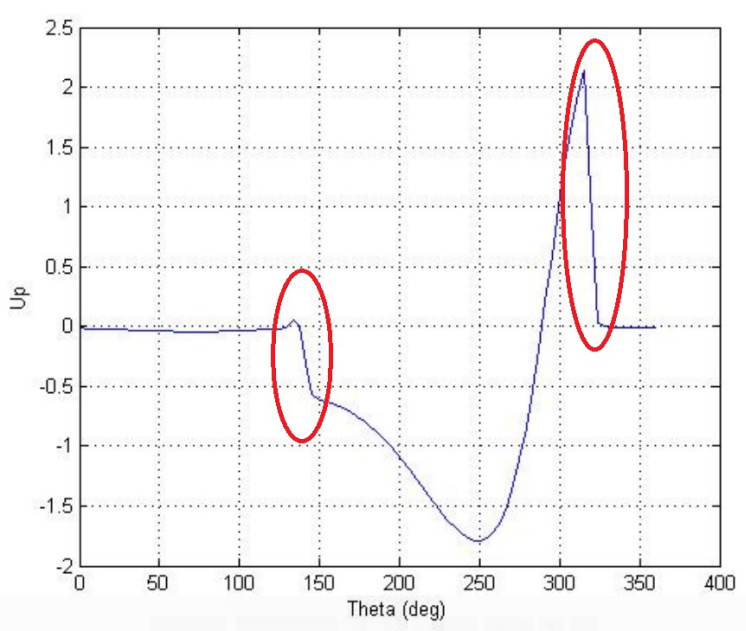

(b) mid-plane $U_{p}$ profile

Figure 4.5: Erroneous jump at the boundaries between cavitation and uncavitated regions

\subsection{Results}

In this section, a few example bearing cases are analyzed and discussed. SLEEVEBRG results are compared and contrasted with other codes, as well as being validated against CFD results. All the results presented in this section are obtained based on an error convergence threshold of $3 e-4$ for pressure calculations and $5 e-4$ for force calculations.

\subsubsection{Water Bearing Pad Analysis}

Armentrout et al. [50] modeled a single pad of a four-pad tilting pad bearing using CFD analysis, Fig. 4.6. In their investigation, they explored different operating aspects of a water lubricated pad. Laminar and turbulent regimes with and without inertia effects were considered in their study. Results were used to modify the Elrod and Ng's turbulence model used within the ROMAC code MAXBRG. The same approach is used here to tune Constantinescu's model implemented within SLEEVE- 


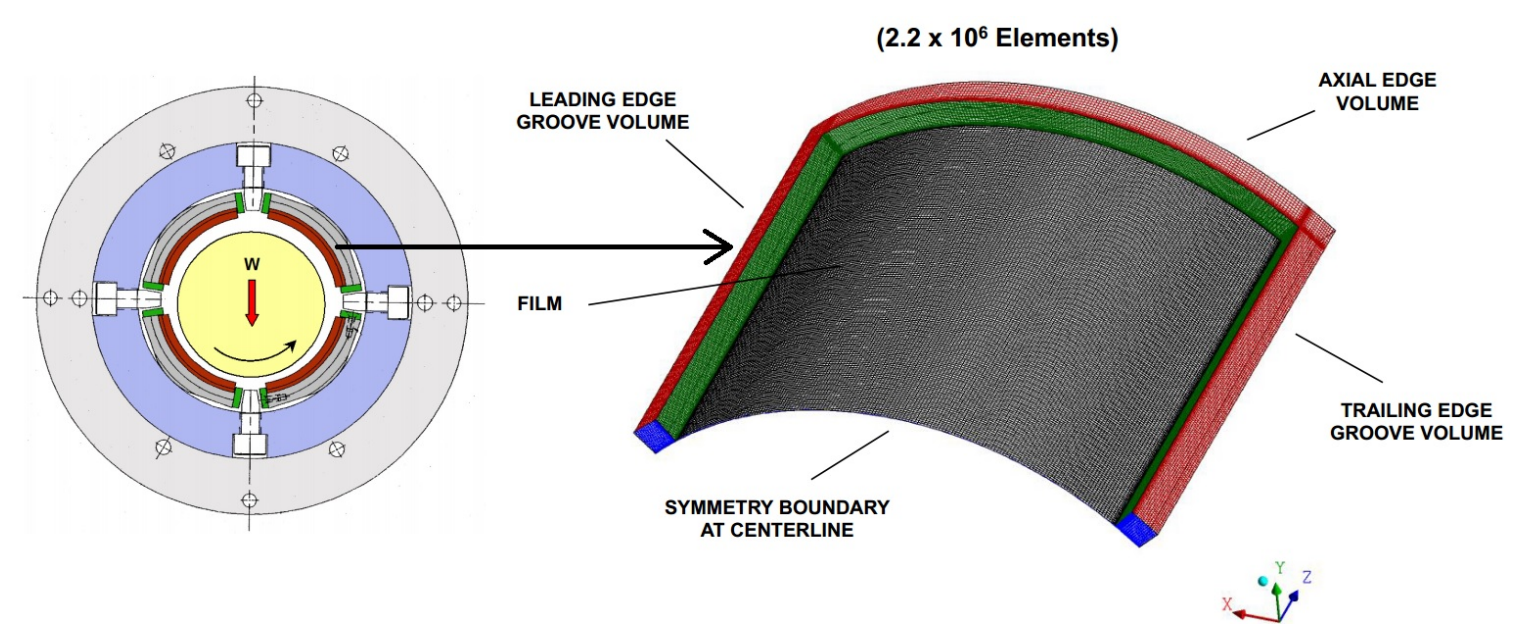

Figure 4.6: CFD model of a single pad of a tilting pad water bearing by Armentrout et al. [50]

BRG. Table. (4.1) lists the pad, and water characteristics and the operation condition.

Maximum Reynolds number and reduced Reynolds number for the operation of the pad are 20433.5498 and 20.43, which ensures the presence of turbulence and therefore significant inertia effects. The starting point is to validate SLEEVEBRG's solver by comparing its solution of the traditional Reynolds equation (i.e., laminar regime without inertia effects) with the solutions from CFD and MAXBRG. Figure (4.7) shows $100 \%$ matching between the three approaches. Note that to obtain these results, the CFD analysis was conducted with turbulence options turned off and water density modified. MAXBRG was also run by manually increasing the turbulence threshold so that laminar assumptions were maintained. In addition, since Elrod and Ng's model does not include an inertia effect, all MAXBRG's results are insensitive to inertia.

For the results depicted in Fig. (4.8), laminar operation is once again enforced, however, the inertia effects are included. As can be observed in this figure, implementing the unmodified Constantinescu's model, Eqs. 2.64 2.70), yields large unre- 
Table 4.1: Water tilting pad bearing [50]

\begin{tabular}{|c|c|c|}
\hline Journal Diameter & $12(\mathrm{in})$ & $304.8(\mathrm{~mm})$ \\
\hline Axial Length & $15(\mathrm{in})$ & $381(\mathrm{~mm})$ \\
\hline Radial clearance & $0.006(\mathrm{in})$ & $0.1524(\mathrm{~mm})$ \\
\hline Pad Arc Length $(\mathrm{deg})$ & 80 & \\
\hline Lubricant Density & $9.3 \mathrm{e}-5\left(\mathrm{lbf} \mathrm{s} \mathrm{s}^{2} / \mathrm{in}^{4}\right)$ & $993.881\left(\mathrm{~kg} / \mathrm{m}^{3}\right)$ \\
\hline Lubricant Viscosity & $5.0 \mathrm{e}-8\left(1 \mathrm{bf} \mathrm{s} / \mathrm{in}^{2}\right)$ & $3.4474 \mathrm{e}-4(\mathrm{~Pa}-\mathrm{s})$ \\
\hline Lubricant Supply Temperature & $120\left({ }^{\circ} \mathrm{F}\right)$ & $48.89\left({ }^{\circ} \mathrm{C}\right)$ \\
\hline Shaft Speed $(\mathrm{rpm})$ & 2000 & \\
\hline Leading edge clearance & $0.00525(\mathrm{in})$ & $0.13335(\mathrm{~mm})$ \\
\hline Trailing edge clearance & $0.00327(\mathrm{in})$ & $0.083058(\mathrm{~mm})$ \\
\hline
\end{tabular}

alistic errors at the leading edge. Recall that Constantinescu proposed two distinct sets of coefficients for his model of convective inertia in the laminar and turbulent regimes, see Eqs. 2.48 2.54). Additionally, the results of these models are depicted in Fig. (4.8). It can be seen that the turbulent inertia model using the $\alpha, \beta, \gamma, \delta$ coefficients in Eq. (2.54) results in lower pressure values compared to the laminar inertia model presented by the $\alpha, \beta, \gamma, \delta$ coefficients of Eq. 2.53). Overall, the inertia effects, for such a large $\mathbf{R e}^{*}$, are shown to be quite significant and tend toward increasing the bearing pressure and corresponding load capacity. In contrast, the CFD results reported by Armentrout et al. [50, which include inertia effects in the laminar regime, demonstrate a pressure drop. This result is contrary to the results obtained from SLEEVEBRG as well as other published results, which show that inertia effects generally act to increase the pressure profile and load capacity of a bearing [3, 14, 90, 91, 92].

DiPrima and Stuart studied the flow between long eccentric rotating cylinders using a modified bipolar coordinate system [92]. This method, although mathematically cumbersome, enabled them to capture the film curvature and convective inertia 


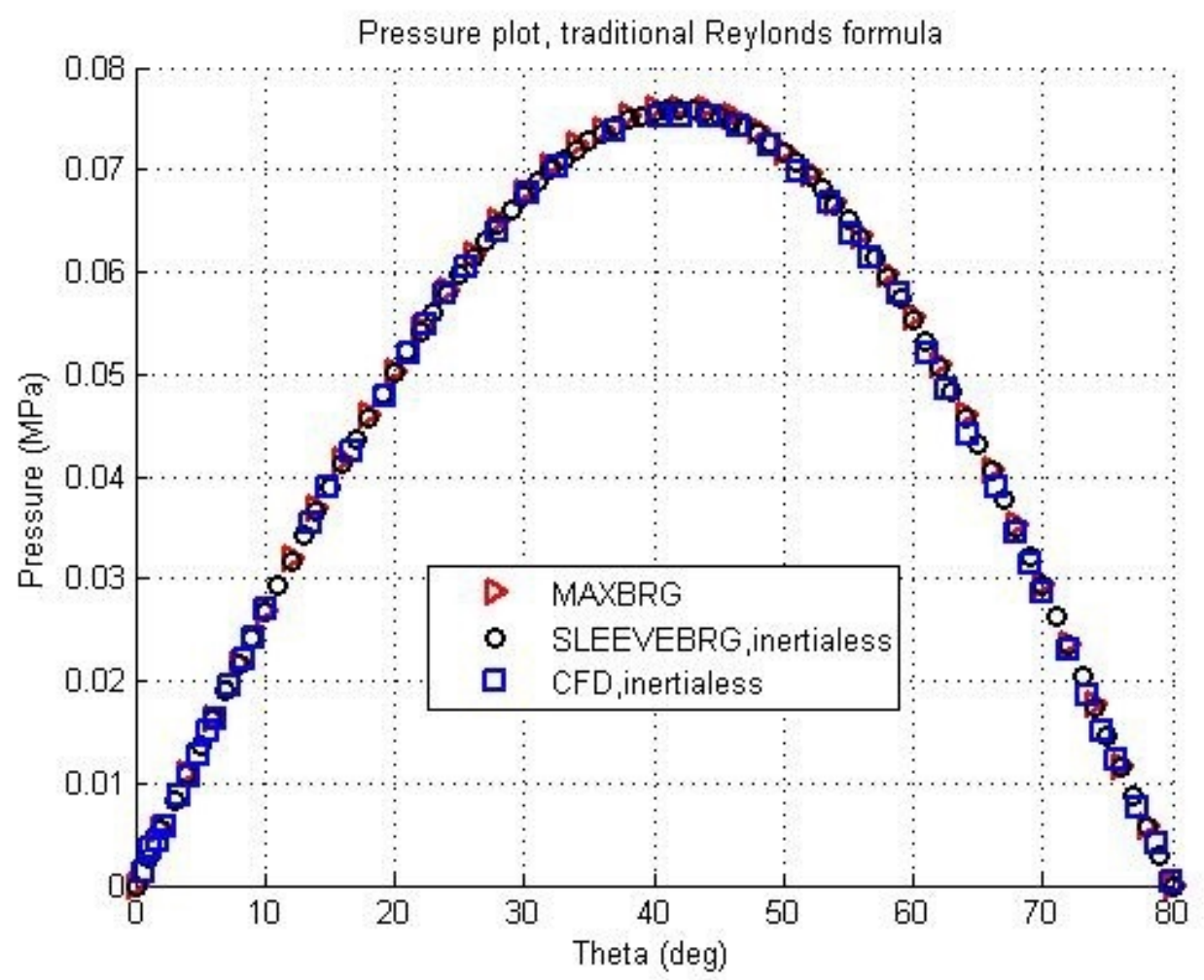

Figure 4.7: Pressure profile of the mid-plane of a single pad. Laminar regime without convective inertia. CFD results from [50]

effects in the solution of Navier Stokes equations. Dai et al. compared the results of the bipolar lubrication theory proposed by DiPrima and Stuart with the results from direct Navier-Stokes and Reynolds equations solutions for Sommerfeld and Gumbel boundary conditions [91]. They tabulated their results and studied the film curvature and convective inertia effects. In this paper, it is shown that the Sommerfeld number, the inverse of nondimensional load capacity, decreases with the increase of the Reynolds number. In other words, load capacity increases with increased Reynolds number.

Tichy and Bou-Said studied finite length bearings operating in the laminar Regime and included convective inertia effects [3]. Their results depicted in Fig. (4a) of [3] exhibit an increase in the pressure profile with Reynolds numbers. In their paper, 


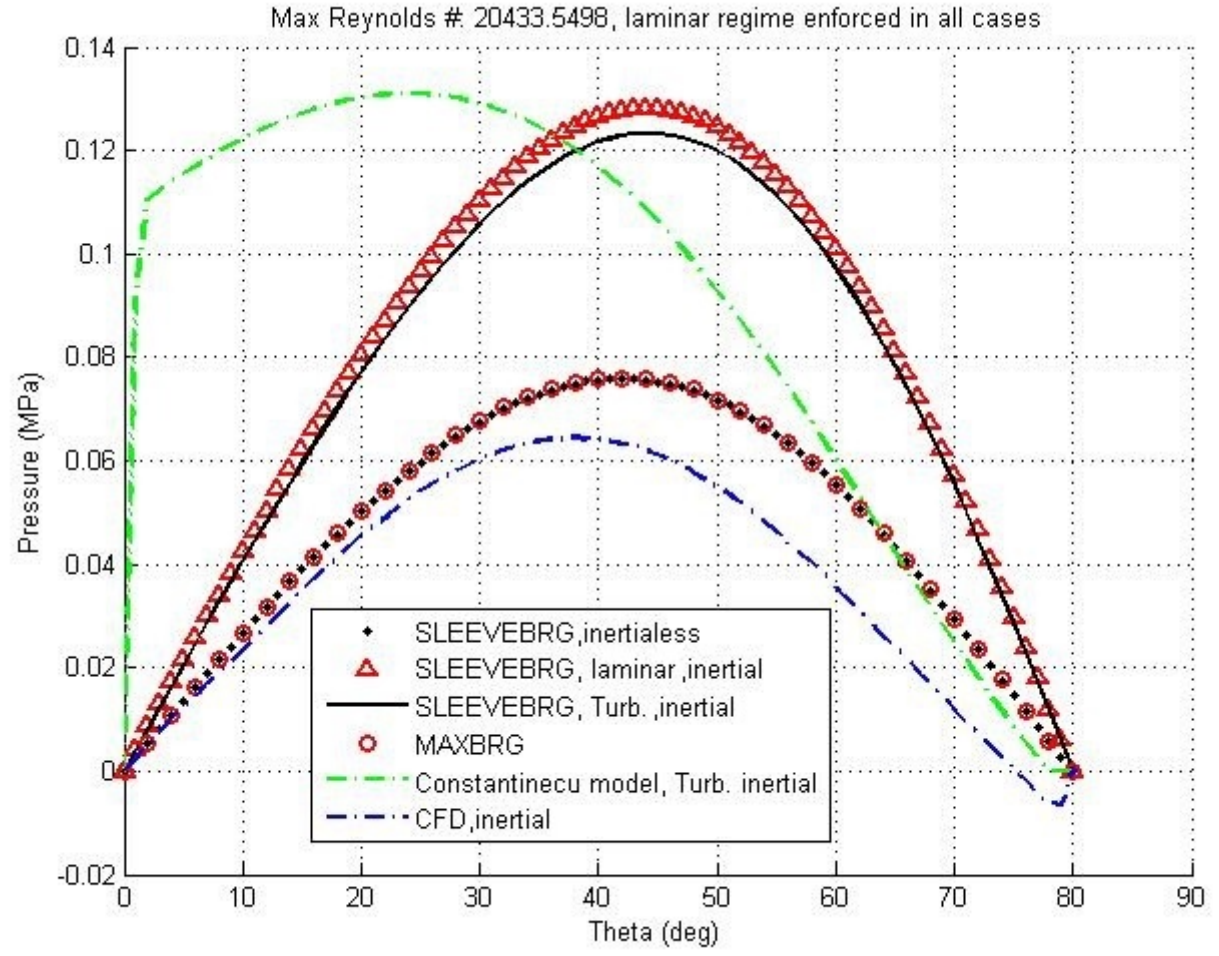

Figure 4.8: Pressure profile of the mid-plane of a single pad. Laminar regime with convective inertia. SLEEVEBRG results based on laminar and turbulent convective inertia models. CFD results from [50] 


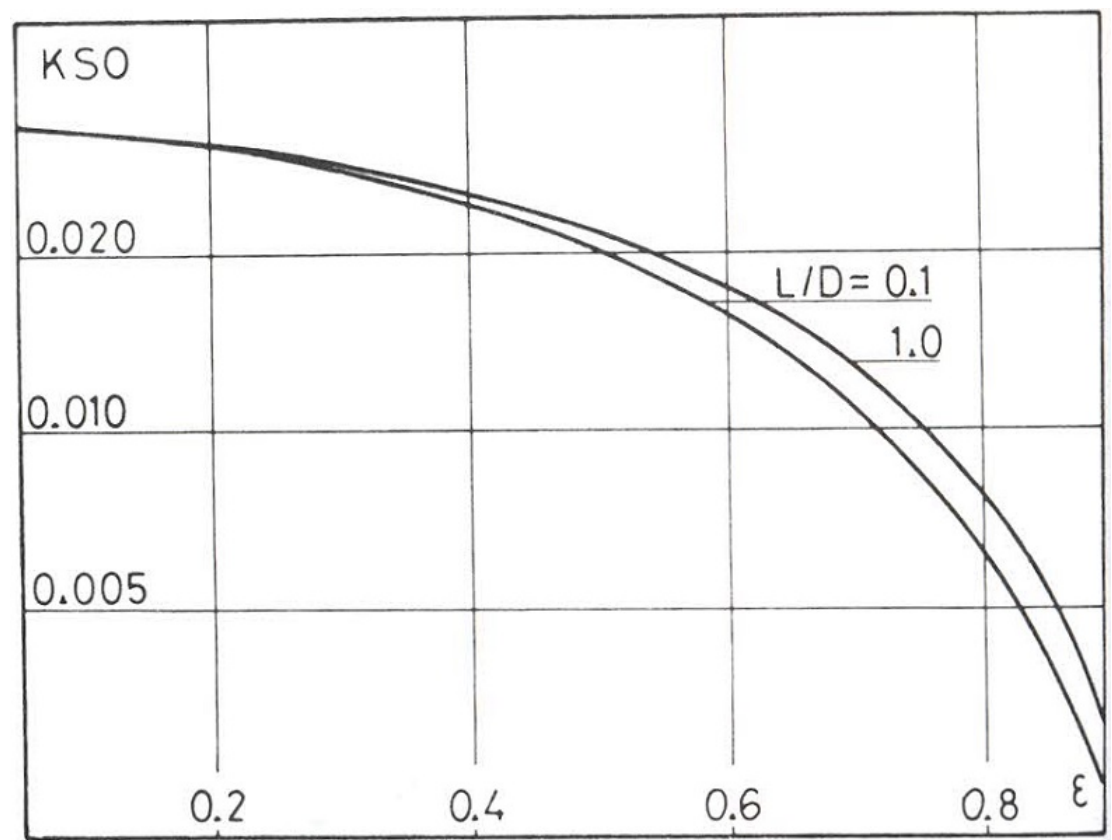

Figure 4.9: Correction to the inverse of Sommerfeld number, Fig. (1) of [14]

Tichy and Bou-Said based their analysis on the well-established findings of Constantinescu [35]], that the inclusion of convective inertia does not affect the shape of the flow velocity profile, but only its average magnitude.

Reinhardt and Lund adopted a perturbation method to solve momentum equations with the inclusion of inertia terms [14]. In their approach, which is conceptually different from Constantinecu's, the unknowns (velocities and pressure) are assumed as a first-order perturbation solution with respect to reduced Reynolds number, $\mathbf{R e}^{*}$, as

$$
\bar{A}=\bar{A}^{0}+R e^{*} \bar{A}^{1}+O\left(R e^{* 2}\right)
$$

which is only valid for applications with $\mathbf{R e}<1$. Reinhardt and Lund showed in Fig. 4.9 that the inclusion of convective inertia terms yields an increased load capacity.

Finally, Gandjalikhan Nassab [90] studied the effect of lubricant inertia on the be- 


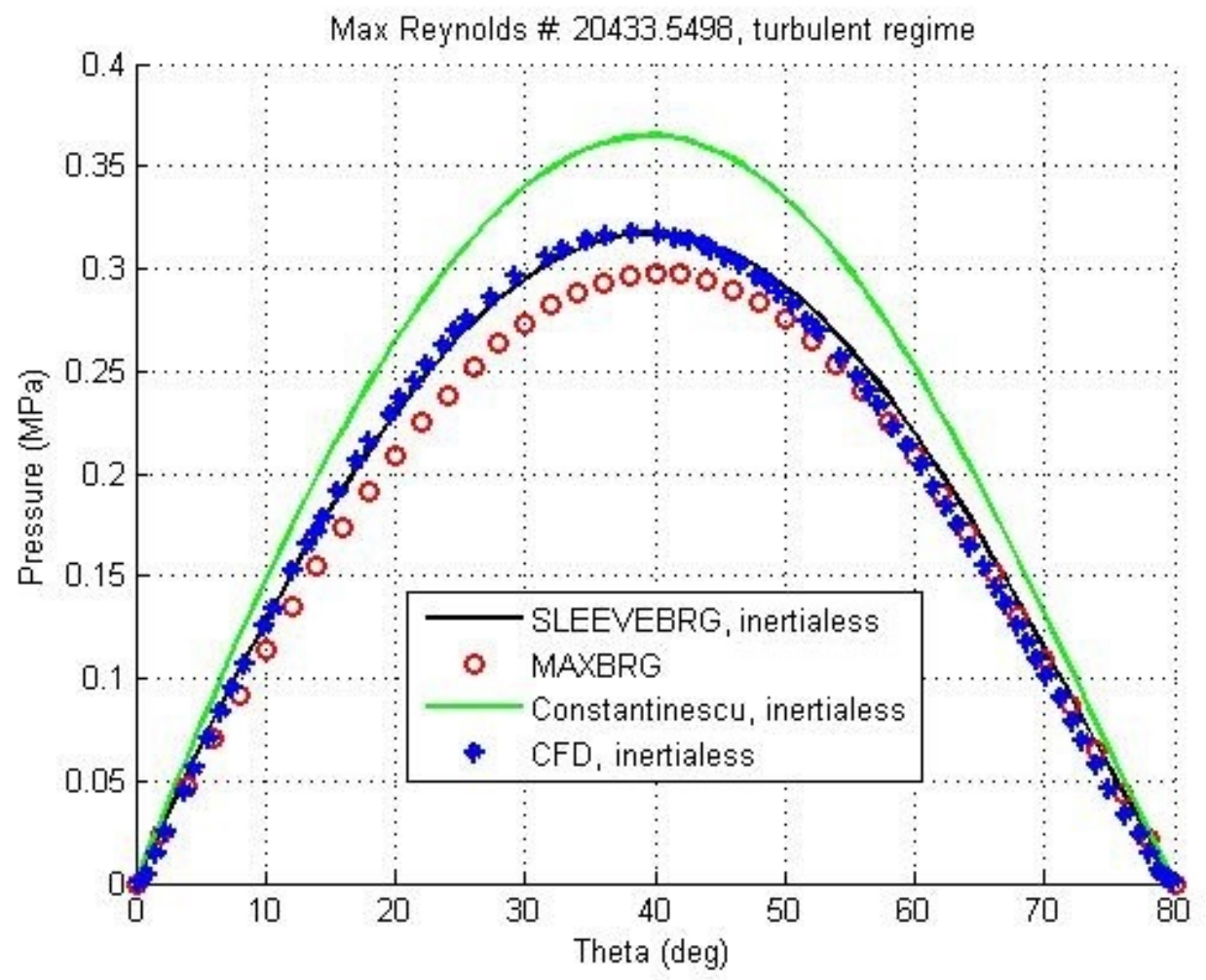

Figure 4.10: $\quad$ Pressure profile of the mid-plane of a single pad operating in the turbulent regime without inertia. CFD results from [50]

havior of journal bearings. Using CFD analysis, he conducted a laminar, three dimensional, thermohydrodynamic study of journal bearings based on the exact governing equations. Experimental data was used in this study to validate the computational results, and the analysis concluded that convective inertia yields higher bearing load capacity.

Therefore, based on the research studies discussed above, the trend that SLEEVEBRG demonstrates (depicted in Fig. (4.7)), that inertia effects generally tend to increase the load capacity of journal bearings operating in the laminar regime is deemed to be correct.

Figure 4.10 illustrates the mid-plane pressure profile of the pad shown in Fig. 4.6 operating in the turbulent regime without inertia effects. Constantinecu's original 


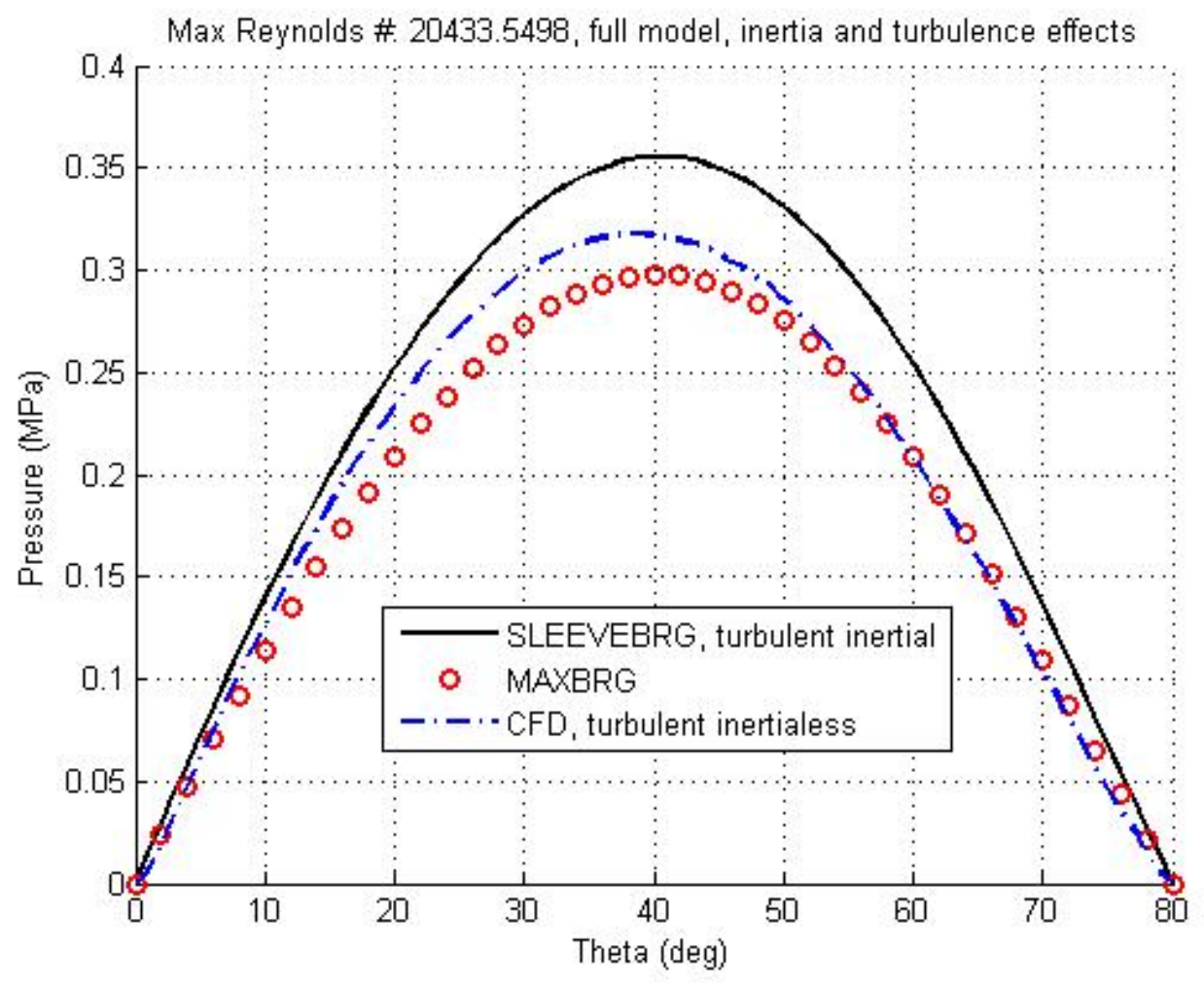

Figure 4.11: Pressure profile of the mid-plane of a single pad. Turbulent regime with inertia. SLEEVEBRG results based on laminar and turbulent convective inertia models. CFD results from [50]

turbulence model Eq. 2.54 overpredicts the maximum pressure, while MAXBRG slightly underpredicts the pressure values predicted by CFD analysis. By using CFD results, Constantinescu's turbulence model was slightly tuned resulting in

$$
k_{x}=12+0.0113 \mathbf{R} \mathbf{e}^{0.9}, k_{z}=12+0.0036 \mathbf{R e}^{0.96}
$$

and this modified model is what is used in SLEEVEBRG. Note that none of the curves shown in Fig. (4.10) includes inertia effects. In order to remove the inertia effects from the CFD results, the CFD analysis was implemented by using an unrealistically low density value for water while the viscosity value was maintained. 
Figure (4.11) demonstrates the results from the full model, i.e., Constantinescu's modified turbulence model and the turbulent inertia model of Eq. (2.78). Unfortunately, no full CFD results in the turbulent regime were reported in [50] in order to compare with these results. From Fig. (4.11), it can be seen that the inclusion of the inertia effects increases load capacity. As indicated before, the inclusion of convective inertia effects act to increase the pressure profile, and therefore, results in an increased load capacity prediction, as depicted in Fig. (4.10). This same trend was also reported in Fig. (8) of [28] by Brunetiere and Tournerie. They observed a radial pressure increase once the convective inertia was included in the analysis of face seals.

\subsubsection{Ferron et al. Bearing}

In this section, we attempt to test the theory developed in this study on a conventional fixed geometry oil bearing and numerically verify the expected results. Since the viscosity of the oil based lubricants is high, the Reynolds number does not increase significantly indicating minor inertia effects. The results in this section verify this theory. However, the computation of the added mass values provided by the new Reynolds equation is a new feature missing in the traditional theories. In the rotordynamic FE model of the system, the bearing added mass values are lumped together with the mass of the shaft element on the bearing. Although, these values for oil bearings are a few percent of the rotor's total mass, they can be larger than the bearing element mass and can therefore alter the rotordynamic properties of the system. Hence, examining the inertia effects of the oil bearing in general can be beneficial by providing a more accurate model of the system.

Ferron et al. in 1983 [93] published a series of comprehensive tests on a single axial groove, plain cylindrical bearing. They measured hydrodynamic pressure 
Table 4.2: Ferron et al. bearing characteristics [93]

\begin{tabular}{|c|c|}
\hline Journal Diameter $(\mathrm{mm})$ & 100 \\
\hline Axial Length $(\mathrm{mm})$ & 80 \\
\hline Radial clearance $(\mathrm{mm})$ & 0.145 \\
\hline Pad Arc Length $(\mathrm{deg})$ & 342 \\
\hline Lubricant Density $\left(\mathrm{kg} / \mathrm{m}^{3}\right)$ & 860 \\
\hline Lubricant Viscosity at $40^{\circ} \mathrm{C}(\mathrm{Pa}-\mathrm{s})$ & 0.028 \\
\hline Lubricant Viscosity at $80^{\circ} \mathrm{C}(\mathrm{Pa}-\mathrm{s})$ & 0.0074 \\
\hline Operating Lubricant Viscosity $(\mathrm{Pa}-\mathrm{s})$ & 0.018 \\
\hline Lubricant Supply Temperature $\left({ }^{\circ} \mathrm{C}\right)$ & 40 \\
\hline Shaft Speed $(\mathrm{rpm})$ & 2000,4000 \\
\hline Load $(\mathrm{kN})$ & $1-10$ \\
\hline
\end{tabular}

distribution, temperature, eccentricity ratio, etc. versus applied load. This bearing, shown in Fig. 4.12, has been widely used to verify numerical analyses [42]. The bearing parameters are listed in Table (4.2). The oil feeding groove is located on top where cavitation occurs, which makes it a suitable example to be analyzed by SLEEVEBRG. The tests were conducted in the low Re numbers and laminar regime. Experimental results and the calculations by MAXBRG were reported by He [42]. In addition, THBRG was run to provide extra numerical results for comparison purposes. MAXBRG and THBRG predict the thermal performance of the bearing and the viscosity drop associated with it, as well. Thermal analysis is beyond the scope of the present SLEEVEBRG-based study; therefore, a mean constant viscosity of $\mu=0.018$ (Pa.s) was assumed for the lubricant film around the entire journal.

Figure 4.13 compares the pressure profile obtained via the three different codes with experimental results. The results obtained from SLEEVEBRG analysis show a very good correlation with experiments. Figure 4.14 illustrates the variation of the journal eccentricity ratio versus applied load. To converge on an equilibrium position, 


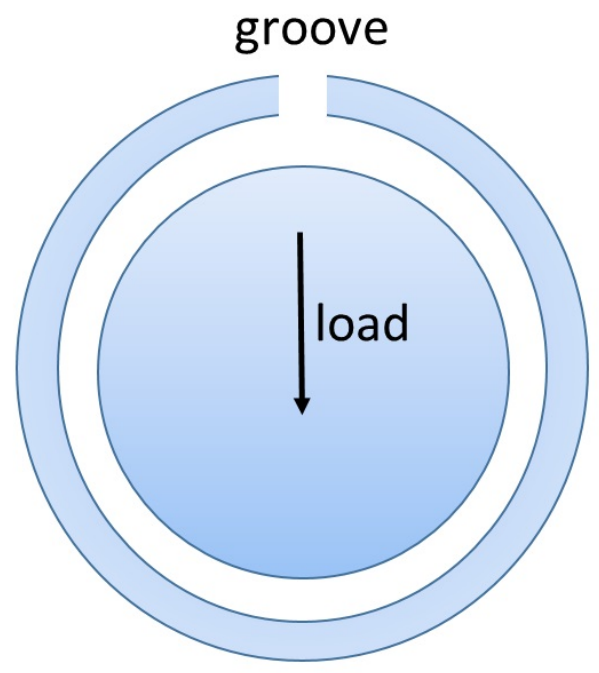

Figure 4.12: Single axial groove Ferron et al. bearing

both codes iterate on the eccentricity ratio and attitude angle (i.e. the horizontal and vertical equilibrium displacements) until the subsequently calculated horizontal and vertical hydrodynamic forces match the external applied forces. It can be observed that the full analysis, with the inclusion of convective inertia, yields nearly the same results as those obtained with convective inertia excluded. This result is due to the low $\mathbf{R e}^{*}$ in oil bearings which makes the inertia effects insignificant.

In order to study the effects of convective inertia on bearing performance, a fictitious $400(\mathrm{Kg})$ rigid rotor is supported by two identical Ferron et al. bearings. The load is distributed evenly between the bearings such that each bearing carries $200(K g)$. The operating speed range is chosen to be from $2000(\mathrm{rpm})$ to $20000(\mathrm{rpm})$, corresponding to $72.55<\boldsymbol{R e}<725.48,0.21<\mathbf{R e}^{*}<2.1039$, and $17.45<\mathbf{S}<174.54$ (Sommerfeld number). This range partially falls into the transition region for $\mathbf{R e}>500$. Figure (4.15) illustrates the variation of journal center location and its eccentricity ratio versus Re. SLEEVEBRG's isoviscous hydrodynamic results are compared to MAXBRG's thermohydrodynamic results. While the inertia effects are shown to be 


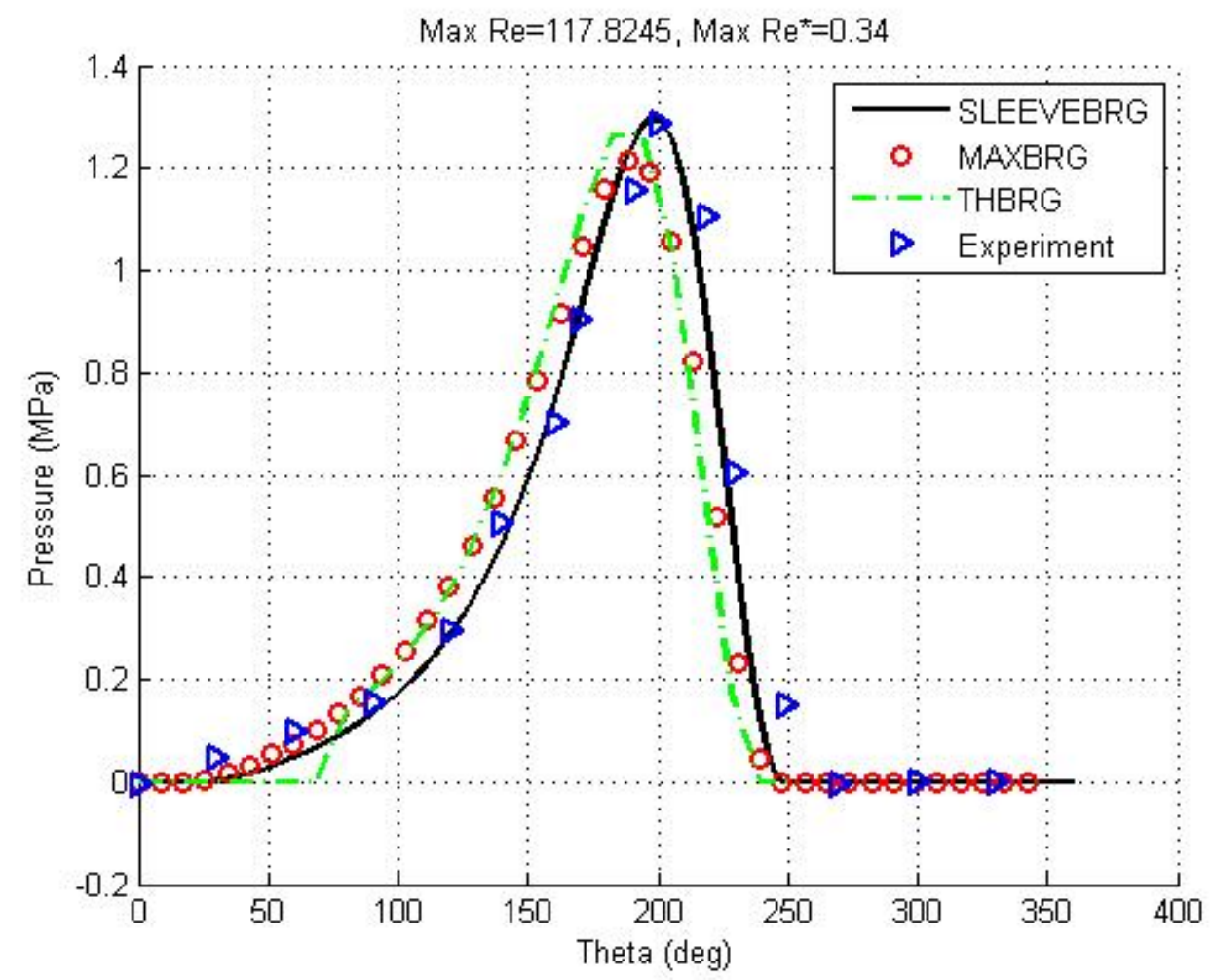

Figure 4.13: Comparison of the mid-span pressure profile of Ferron et al. bearing from experiment, thermohydordynamic analysis of MAXBRG (by [42]) and THBRG, and isothermal hydordynamic analysis of SLEEVEBRG at 2000(rpm), 4000(N) load, and $\mu=0.018$ (Pa.s) 


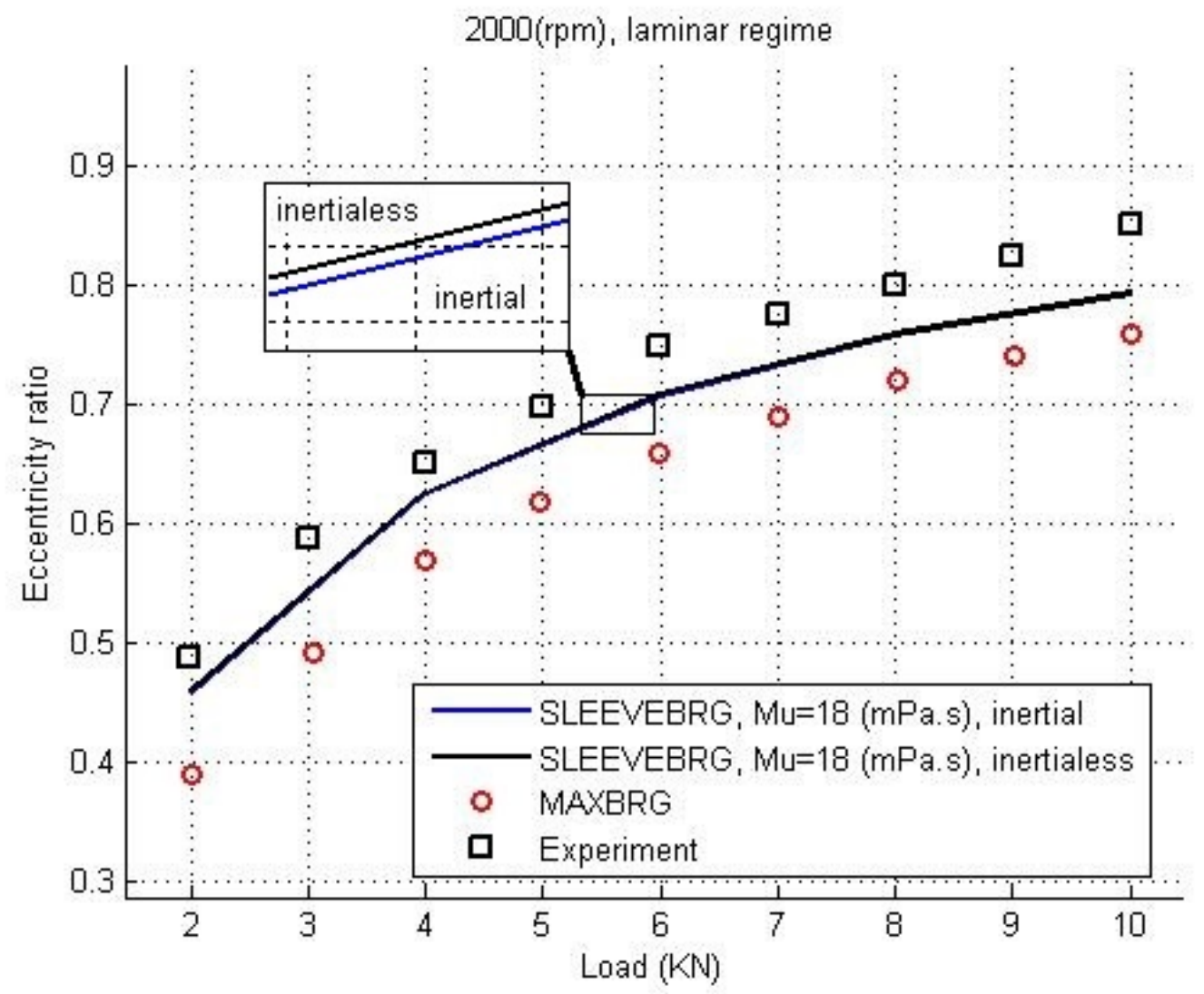

Figure 4.14: Variation of eccentricity ratio vs. applied vertical load, $\omega=2000($ rpm $)$ 


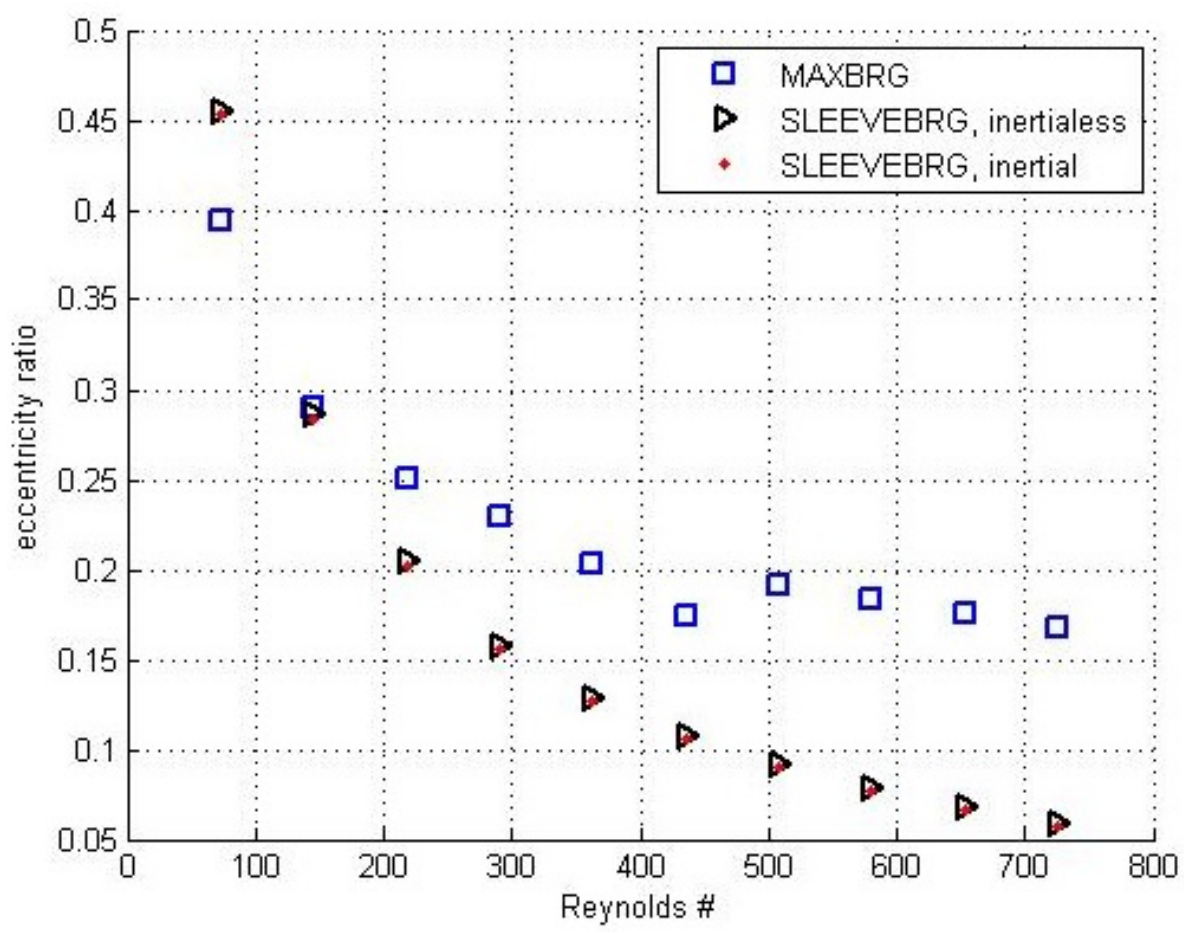

(a) Eccentricity ratio versus Re, jump due to crossing to transition region

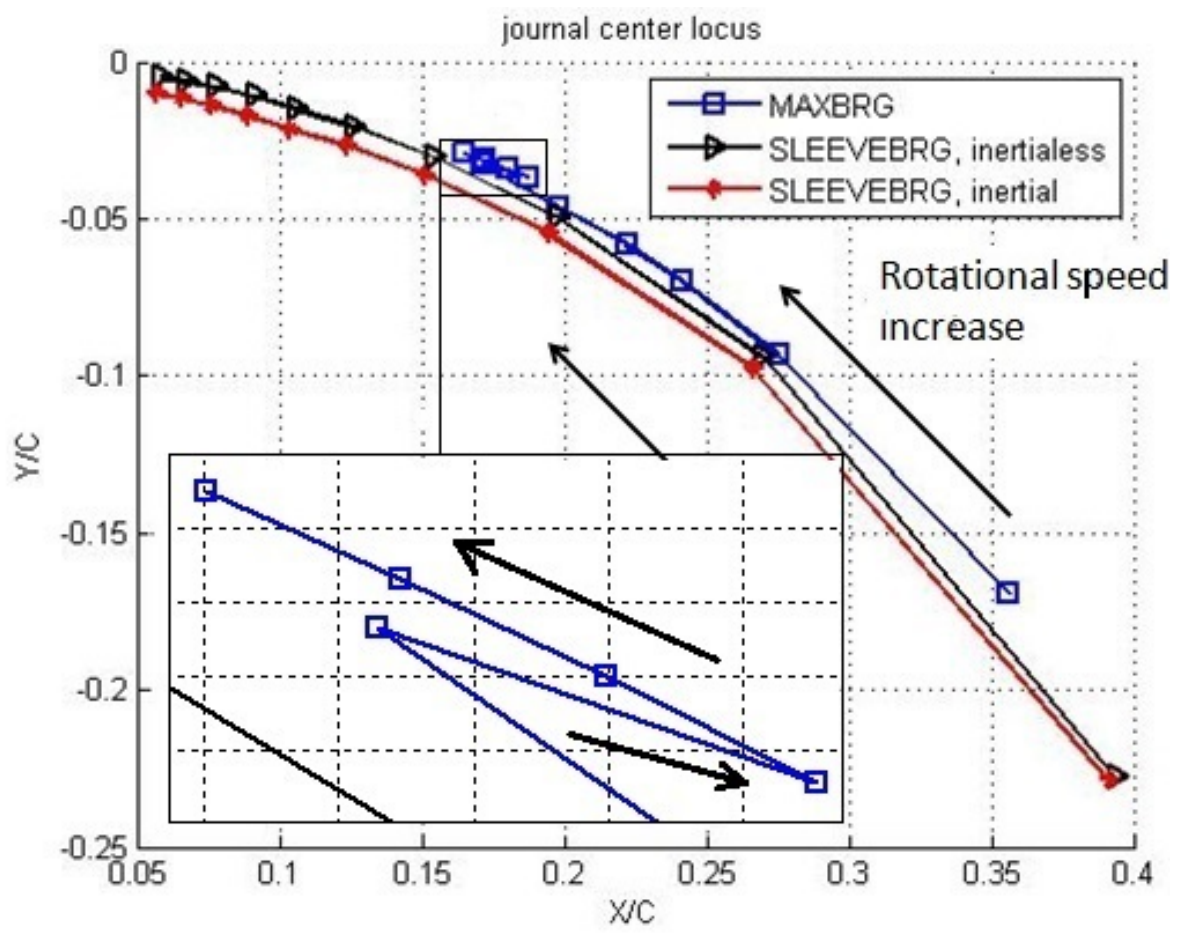

(b) Journal center locus

Figure 4.15: Steady state operation of Ferron et al. bearing with a 200 $(K g)$ load , and $\mu=0.018$ (Pa.s) for SLEEVEBRG 
negligible, thermal effects cause MAXBRG's results to differ from SLEEVEBRG's. The higher the speed, and consequently the higher $\mathbf{R e}$, the larger the difference between MAXBRG's and SLEEVEBRG's results. At high rotational speed, larger power loss and energy dissipation occurs. The lost energy heats up the lubricant and causes its viscosity to drop. Energy loss is higher in the narrower clearance regions and therefore a viscosity gradient in the circumferential direction forms. This justifies the observation that for higher rotational speeds, higher eccentricity ratios are predicted by thermohydrodynamic analysis compared to those obtained from the isothermal/isoviscous analysis of SLEEVEBRG. This observation emphasizes the importance of the thermal analysis, especially at higher rotational speeds for oil bearings. Also, it can be seen from Fig. 4.15 that the onset of the transition regime at $\mathbf{R e}=500$ results in a jump in data which is more pronounced in MAXBRG's results.

Rotordynamic coefficients of the Ferron et al. bearing are plotted in Figs. 4.164.21). As expected, it can be seen that SLEEVEBRG and MAXBRG results vary considerably from each other due to the differences in their underlying analyses. On one hand, the equilibrium position as the location where the perturbation is conducted around differs in the two analyses (i.e., indirect impact on the rotordynamic coefficients), and on the other hand, by inclusion of the thermal analysis in MAXBRG, the viscosity of the lubricant calculated in the circumferential direction may vary considerably due to the temperature gradient, while the isothermal analysis of SLEEVEBRG neglects the thermal effects. Additionally, SLEEVEBRG's inclusion of the convective inertia, by introducing new perturbation terms in the calculations (see Secs. 4.1.2 \& 4.1.3), results in further distinctions between the coefficients obtained from the two analysis methods.

For lower rotational speeds, the heat generated due to the shear power loss is lower 


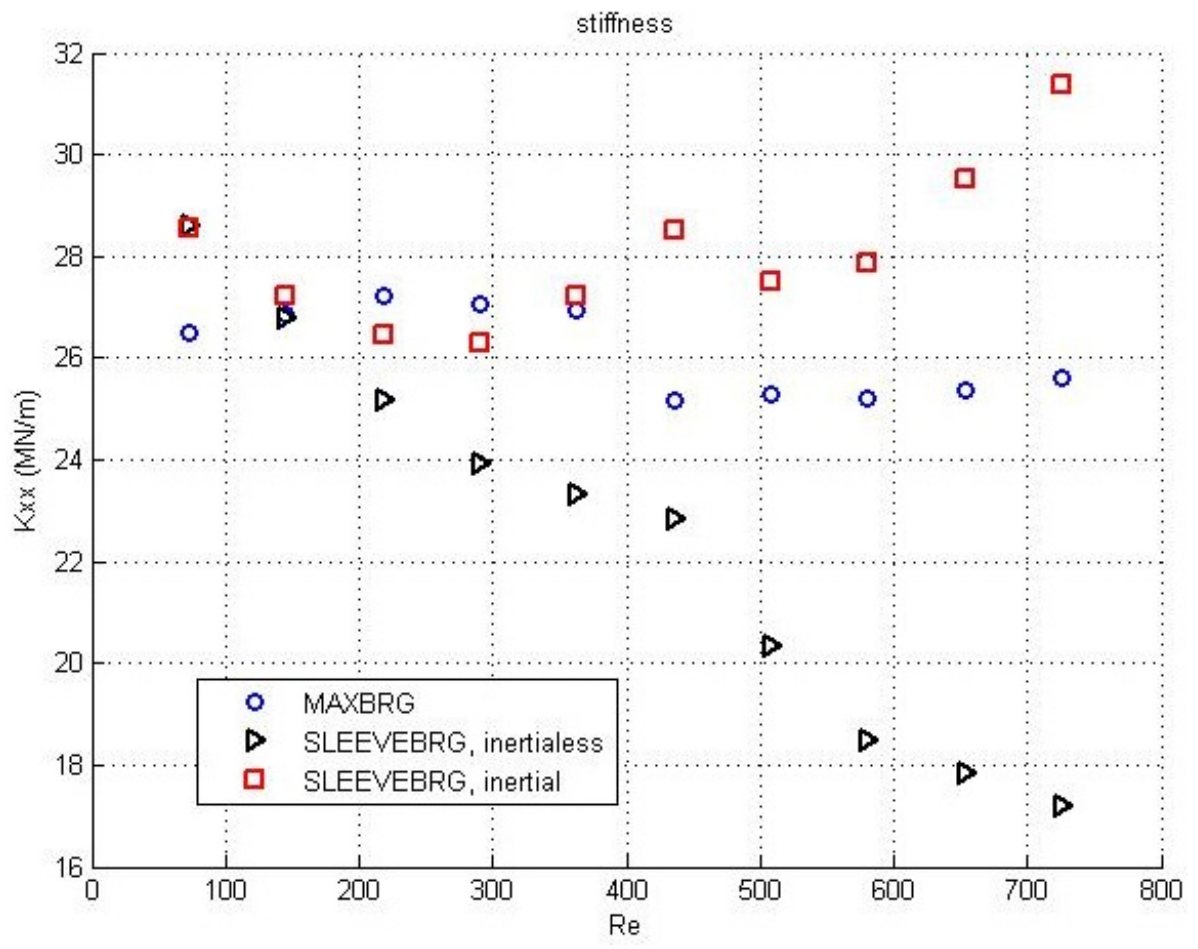

(a) $K_{x x}$

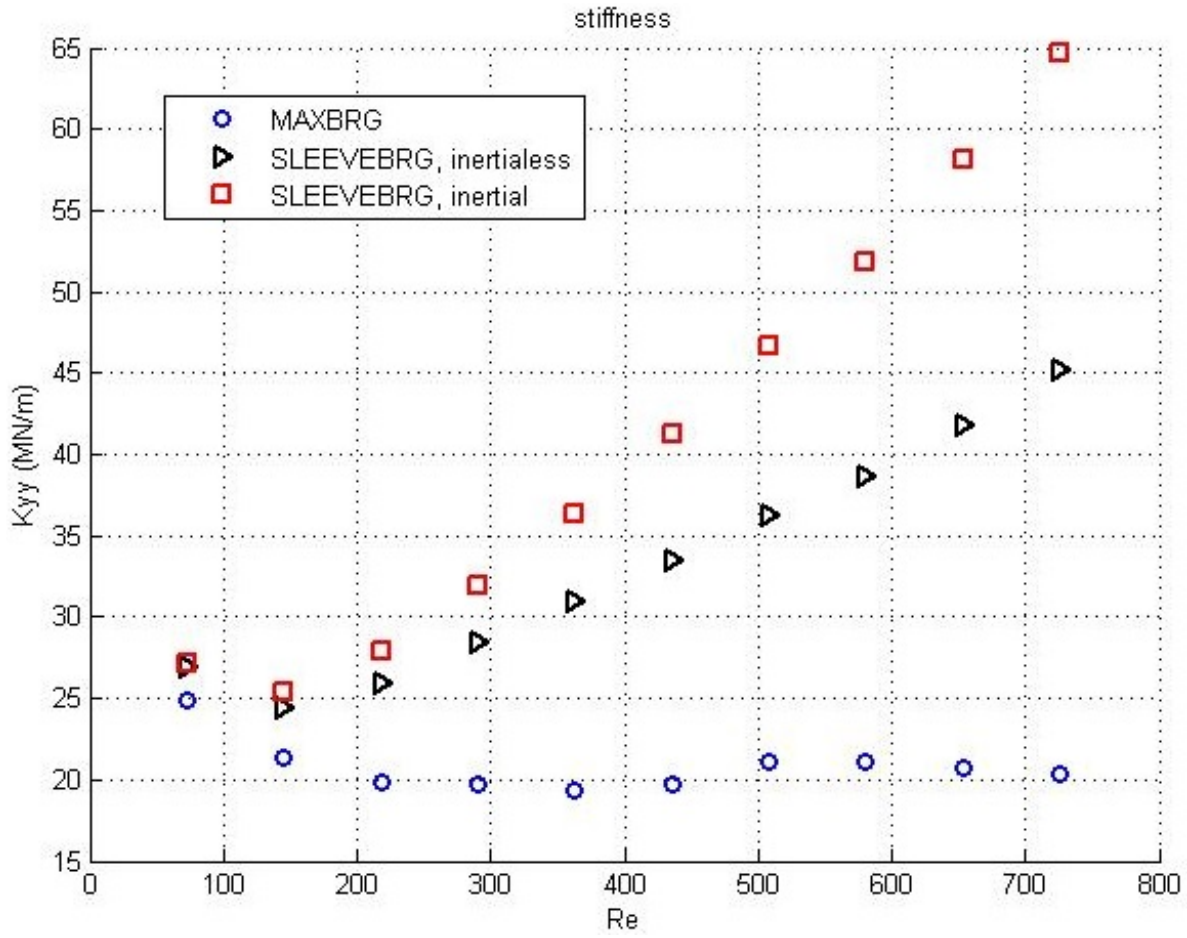

(b) $K_{y y}$

Figure 4.16: Principle stiffness, Ferron et al. bearing with $200(K g)$ vertical load and $2000($ rpm $)<\omega<20000($ rpm $)$ rotational speed 


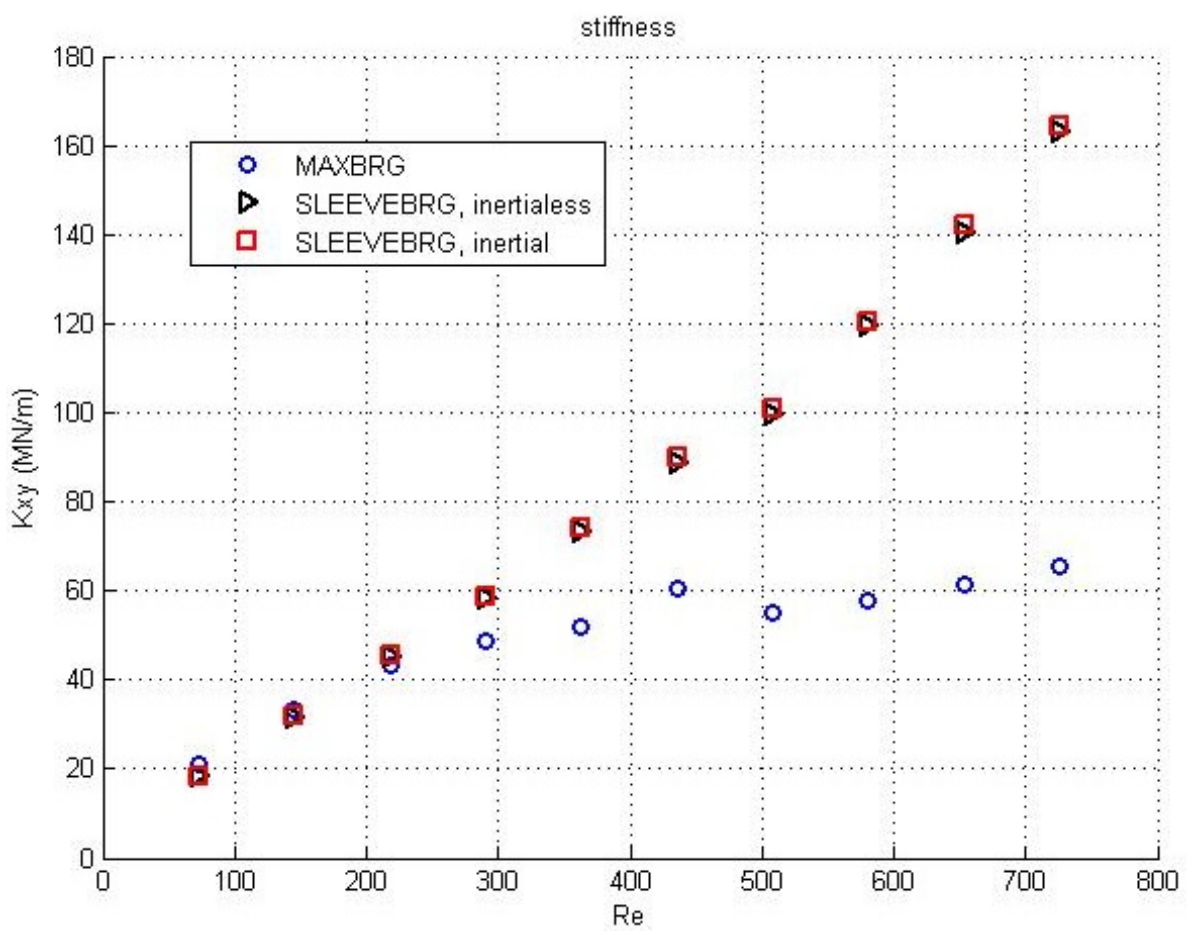

(a) $K_{x y}$

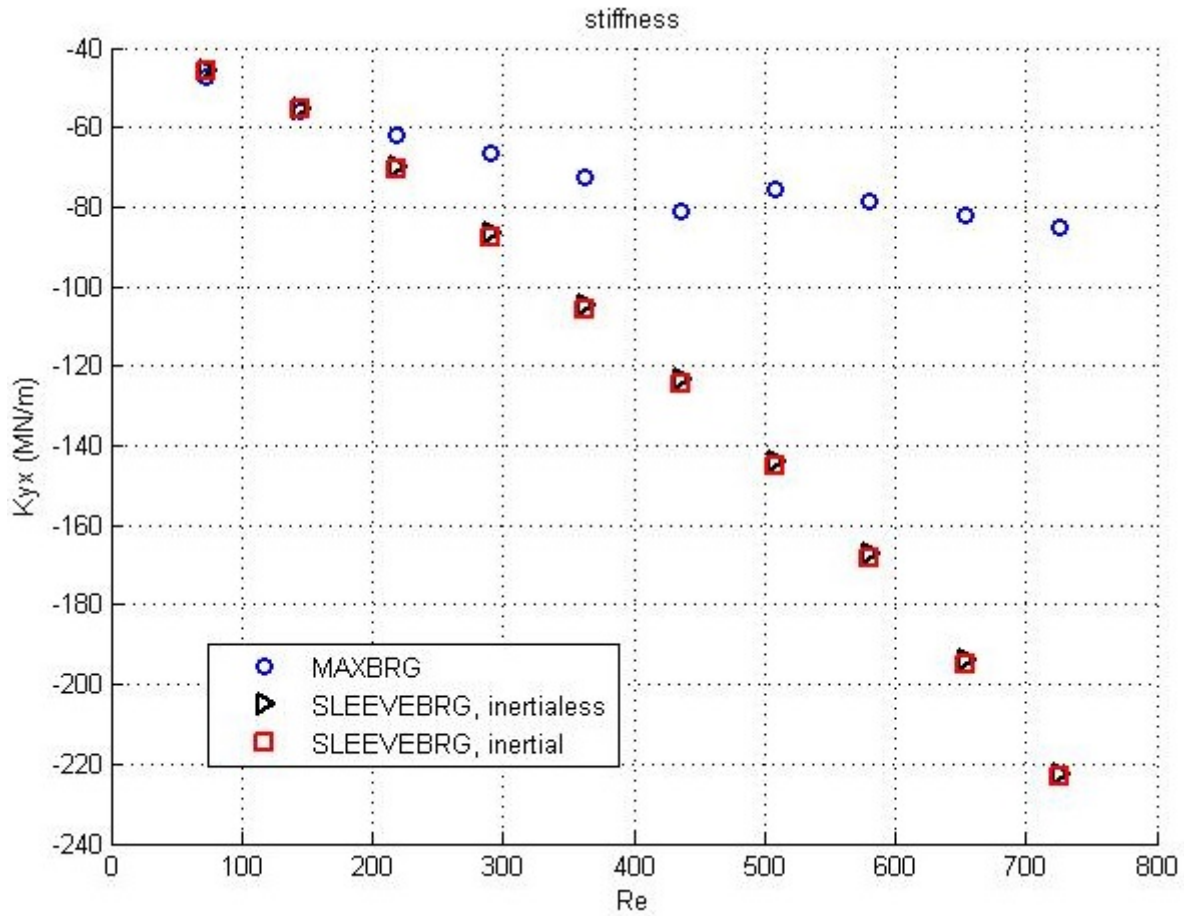

(b) $K_{y x}$

Figure 4.17: Cross-coupled stiffness, Ferron et al. bearing with 200 $(K g)$ vertical load and $2000(\mathrm{rpm})<\omega<20000(\mathrm{rpm})$ rotational speed 


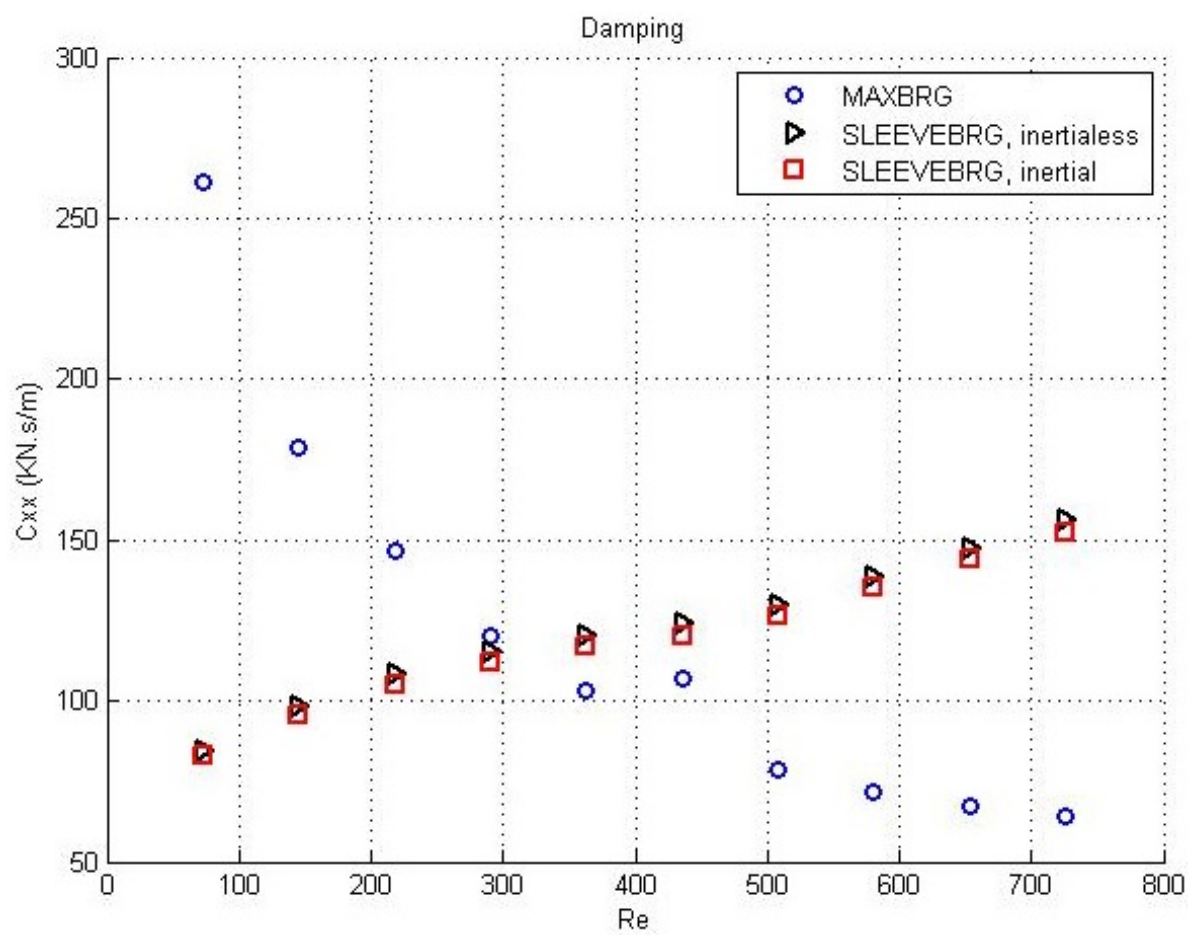

(a) $C_{x x}$

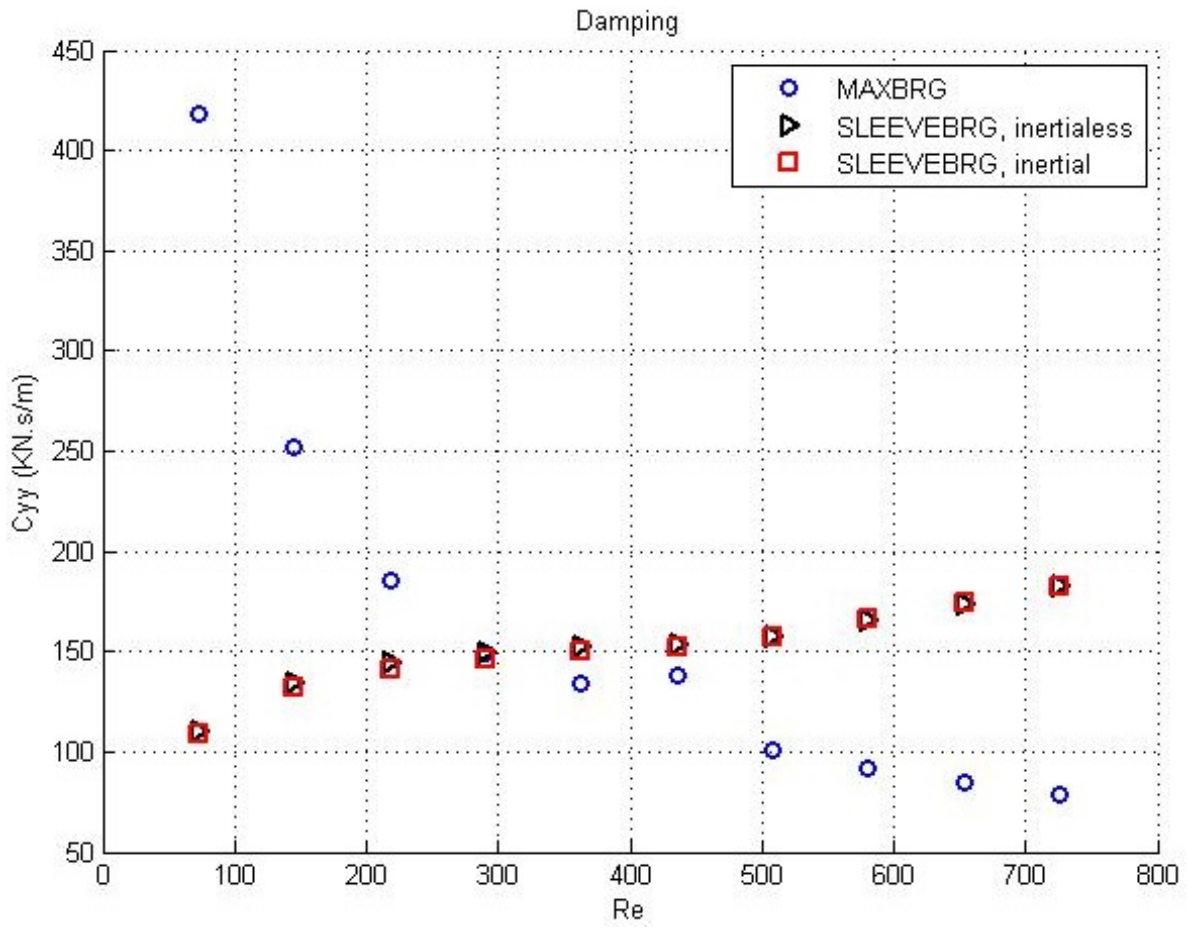

(b) $C_{y y}$

Figure 4.18: Principle damping, Ferron et al. bearing with 200( $\mathrm{Kg})$ vertical load and $2000($ rpm $)<\omega<20000($ rpm $)$ rotational speed 


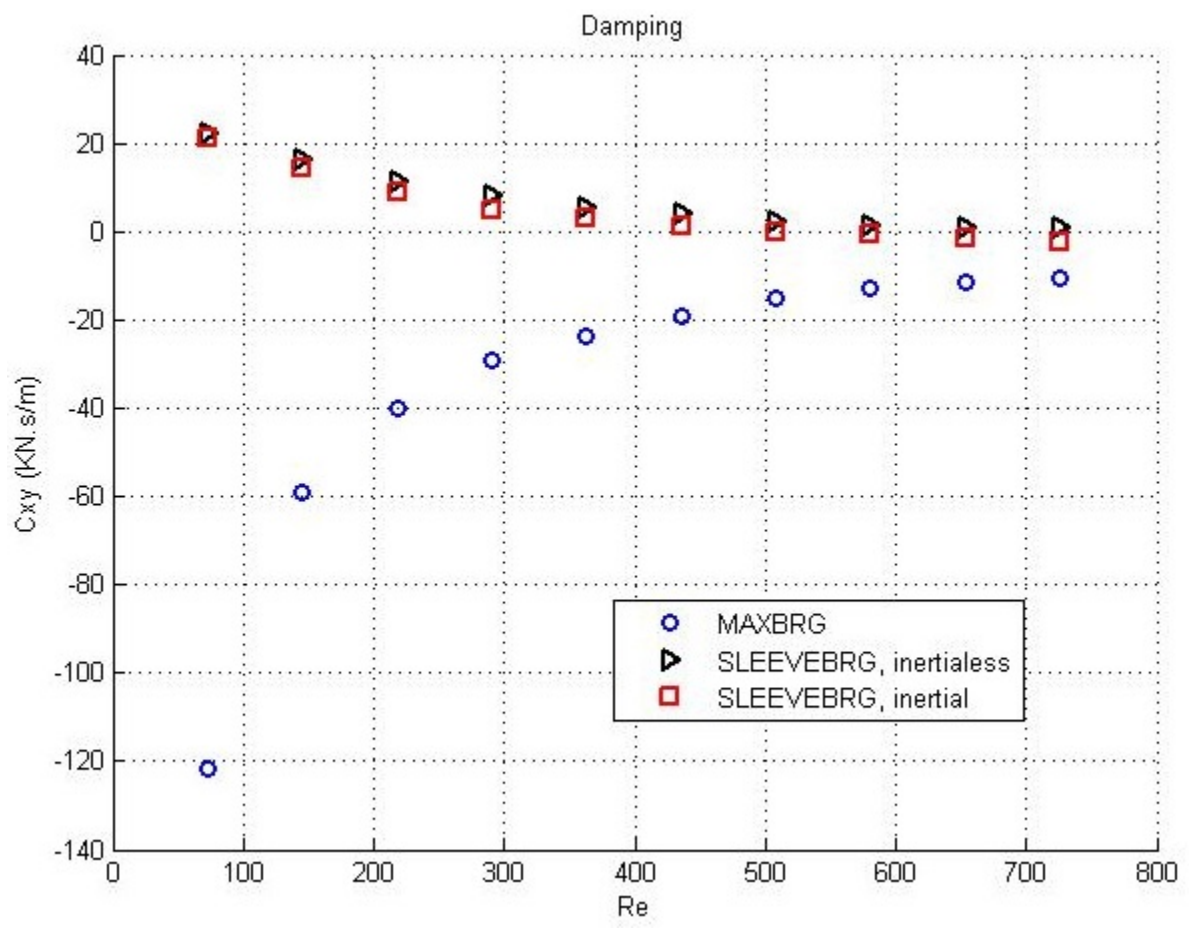

(a) $C_{x y}$

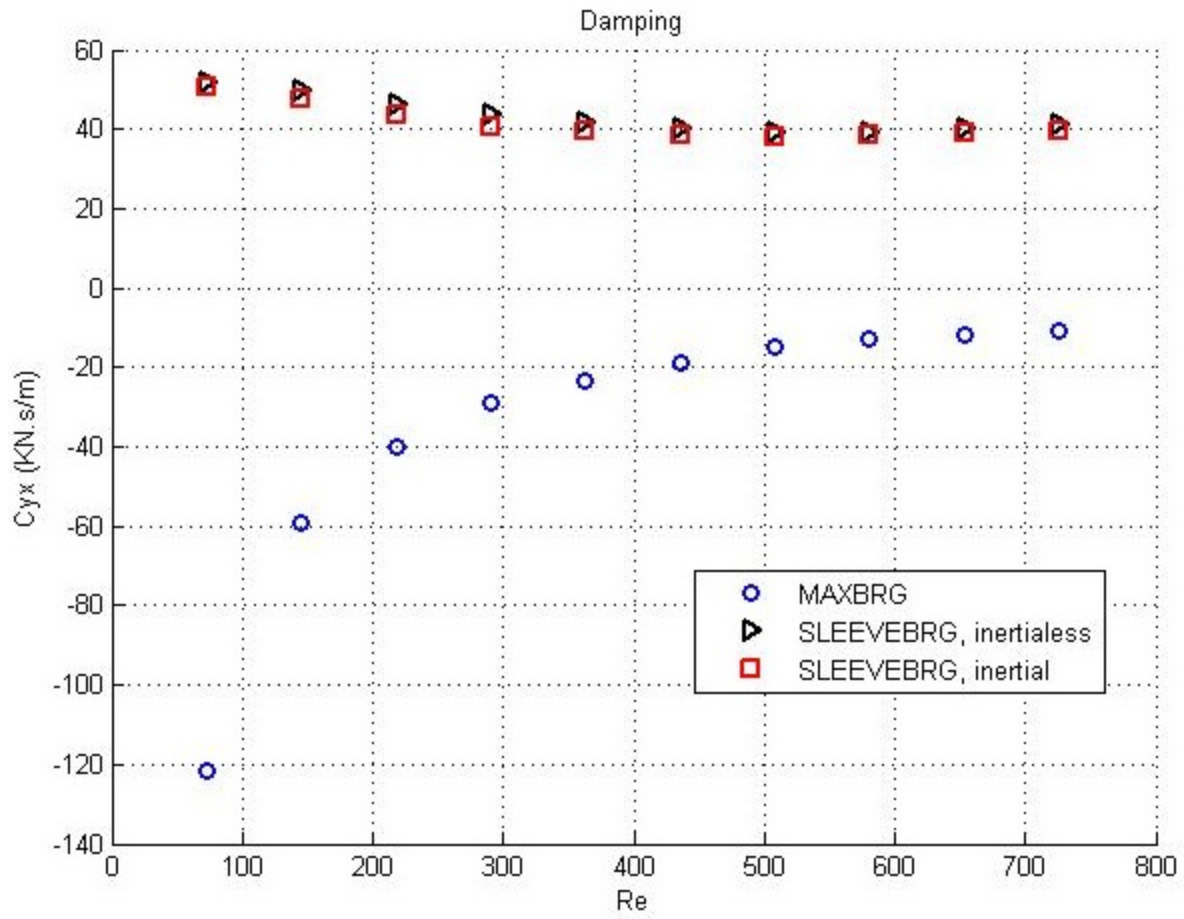

(b) $C_{y x}$

Figure 4.19: Cross-coupled damping, Ferron et al. bearing with 200 $(\mathrm{Kg})$ vertical load and $2000($ rpm $)<\omega<20000($ rpm $)$ rotational speed 


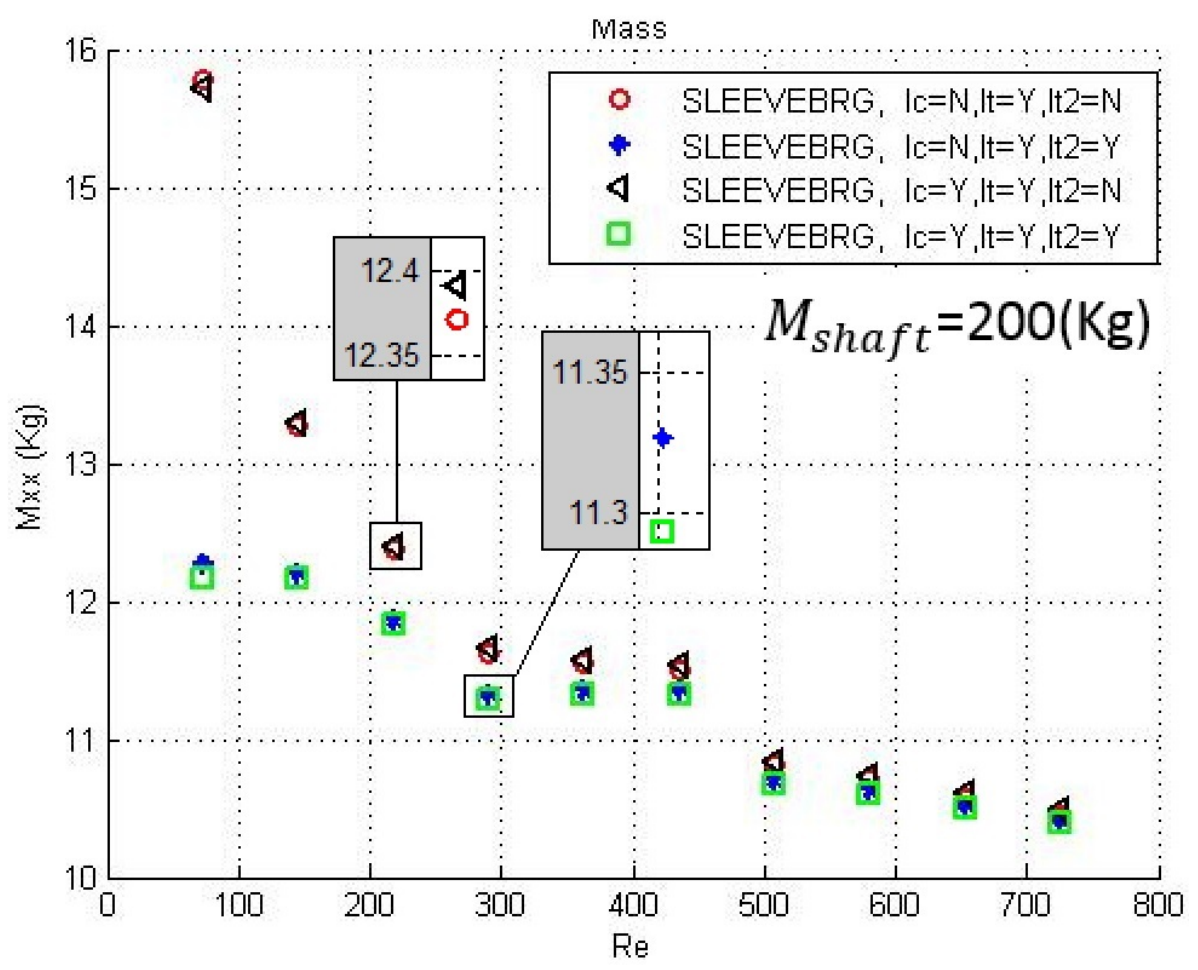

(a) $M_{x x}$

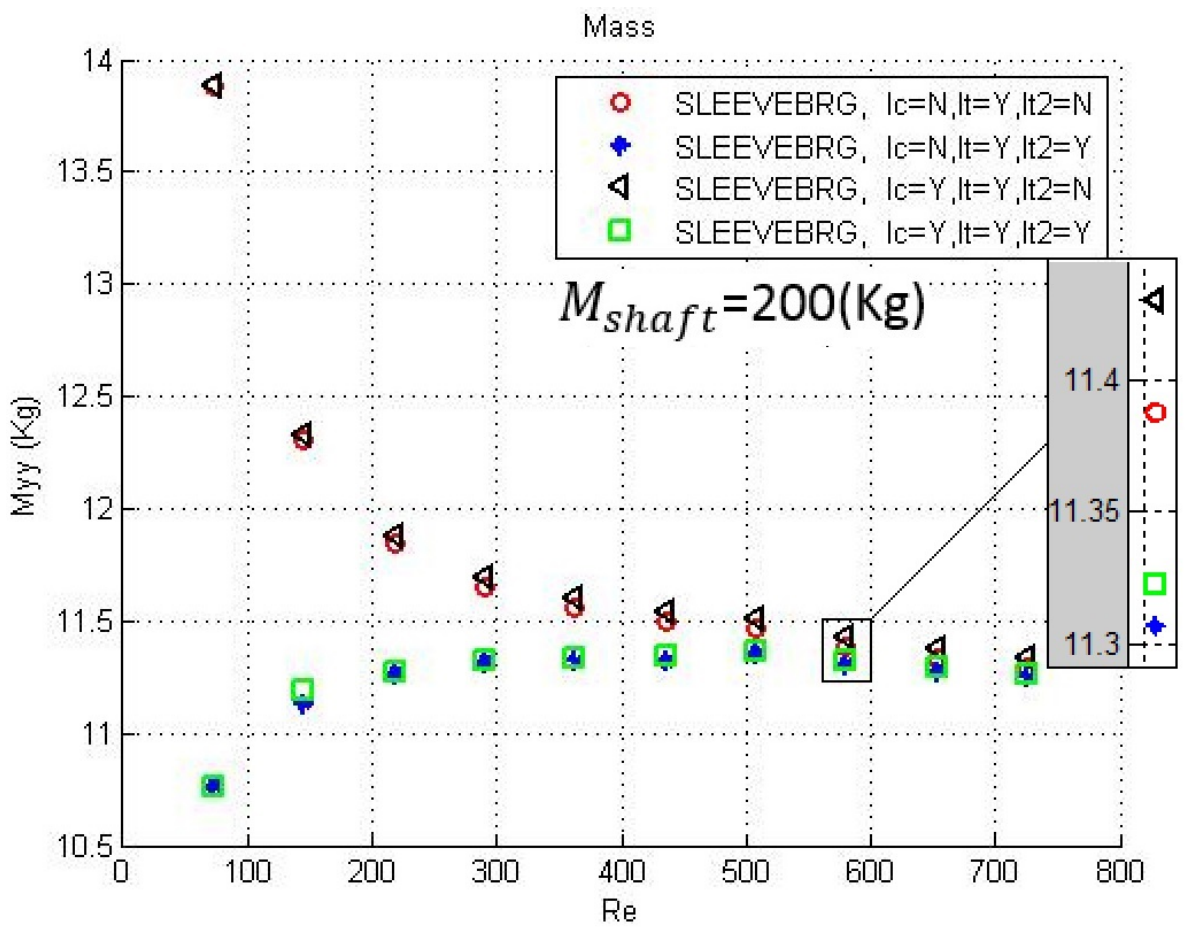

(b) $M_{y y}$

Figure 4.20: Principle added mass, Ferron et al. bearing with 200 $(\mathrm{Kg})$ vertical load and $2000(\mathrm{rpm})<\omega<20000(\mathrm{rpm})$ rotational speed 


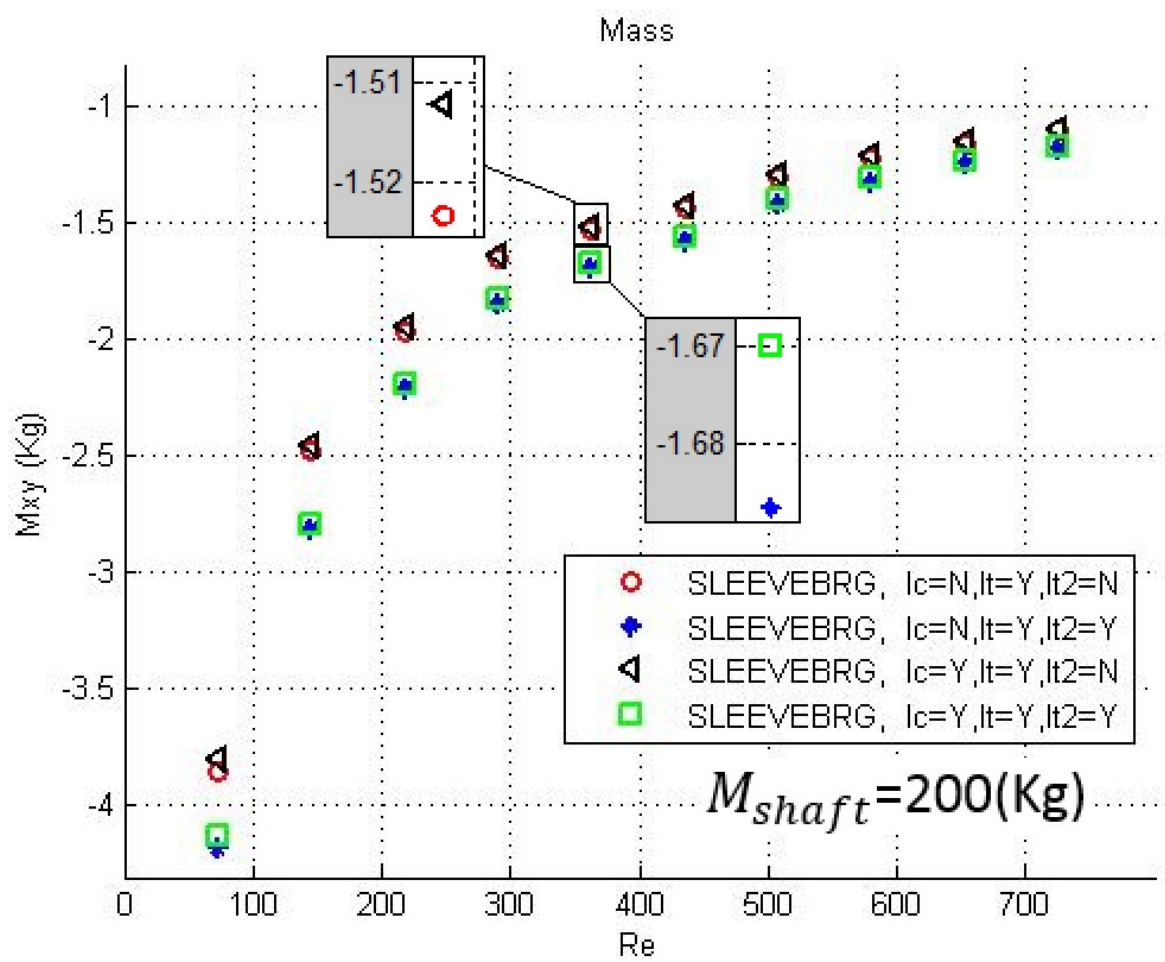

(a) $M_{x y}$

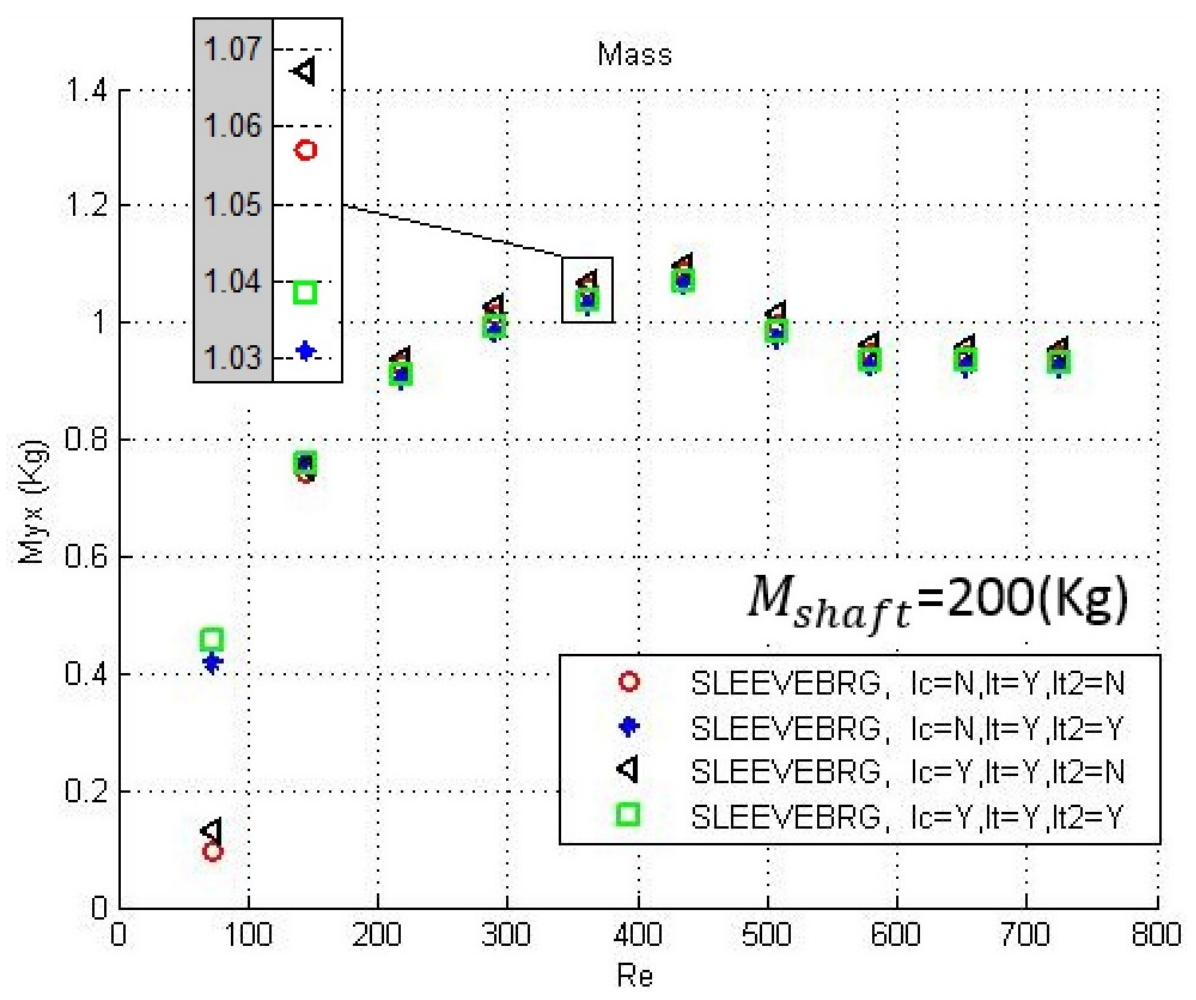

(b) $M_{y x}$

Figure 4.21: Cross-coupled added mass, Ferron et al. bearing with 200 $(\mathrm{Kg})$ vertical load and $2000(\mathrm{rpm})<\omega<20000(\mathrm{rpm})$ rotational speed 
resulting in a smaller deviation from isoviscous condition, and consequently, closer stiffness values are predicted by the two approaches as shown in Figs. (4.16 \& 4.17). In addition, the (absolute) values of the stiffness coefficients, especially the principle stiffnesses, are affected more by convective inertia and are higher once calculated with the inclusion of the inertia effects. Moreover, effects of operating in the transition regime is accented in the form of a jump in the coefficient values at $\mathbf{R e}=500$ in Fig. 4.16(a).

In Figs. $4.18 \& 4.19$ ), it is demonstrated that the damping values are significantly affected by thermal effects, such that the trend, the values, and in some cases even the sign of the coefficients calculated by the two approaches vary from each other. Results from these figures also demonstrate that convective inertia has negligible effects on the damping values.

Figures 4.20 and 4.21 illustrate the added mass variation versus $\mathbf{R e}$ under four different $I_{t}, I_{c}$ combinations based on SLEEVEBRG analysis. In these figures, Ic $=\mathrm{Y}$ and $\mathrm{I} c=\mathrm{N}$ indicate the added mass curves calculated with and without convective inertia effects, respectively. Similarly, It2 $=\mathrm{Y}$ and It2 $=\mathrm{N}$ represent cases with and without the secondary temporal inertia term included. The principle added mass coefficients are roughly an order of magnitude higher than the cross-coupled values, while they are $\sim 10 \%$ of the physical mass of the shaft. It is observed that the convective inertia effects on the added mass coefficients are insignificant. Turning attention to the effect of the secondary temporal inertia term, it can be seen that this term in general lowers the magnitude (i.e., the absolute value) of the calculated added mass. And its effect at the lower $\mathbf{R e}$ applications is greater than for higher $\mathbf{R e}$ applications. The principle added mass coefficients exhibit up to $\sim 15 \%$ fluctuations with the inclusion of the secondary temporal inertia term for low Re values, while the secondary temporal inertia effects on the cross-coupled added mass coefficients 


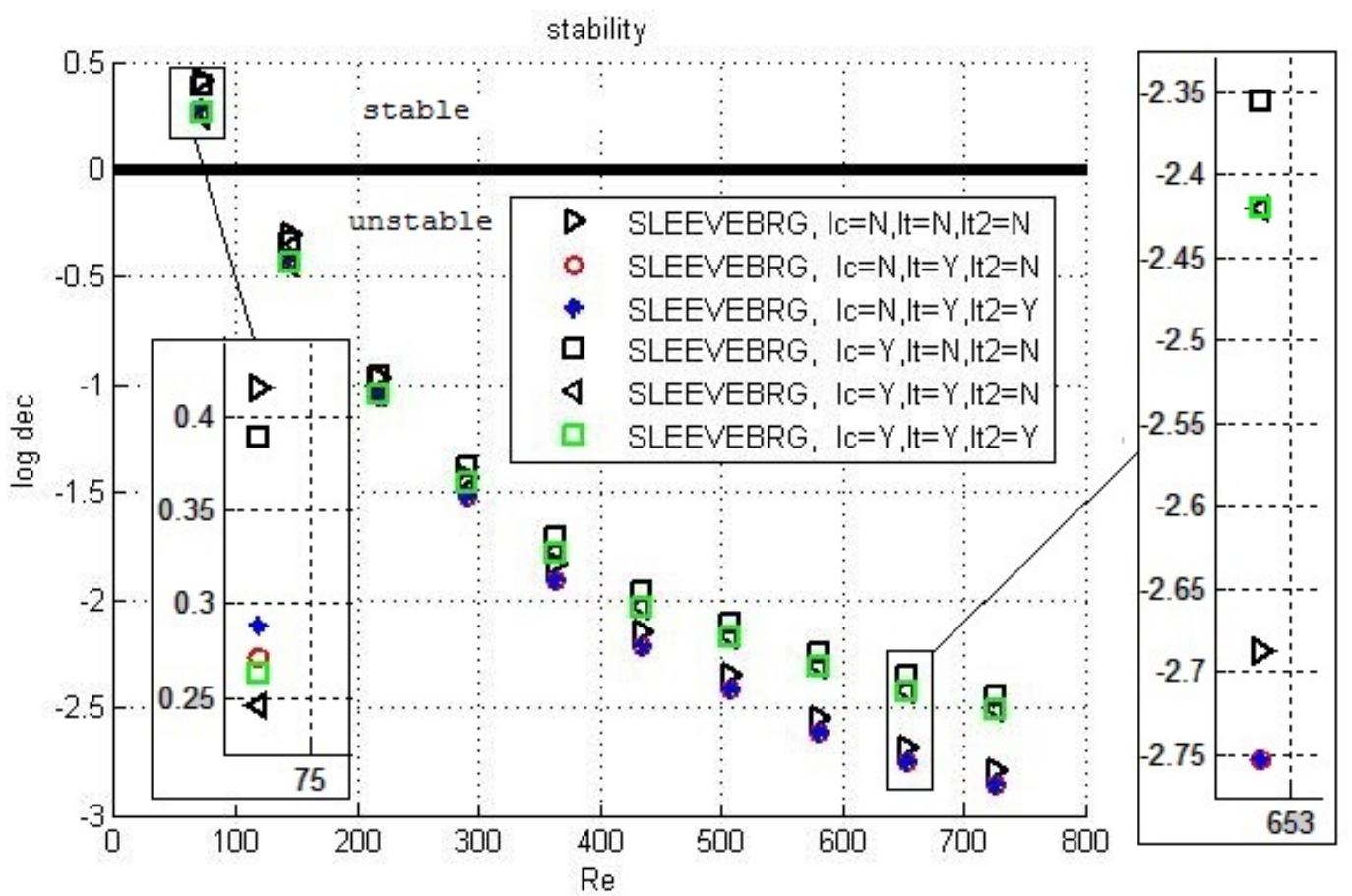

Figure 4.22: Stability of Ferron et al. bearing with $200(\mathrm{Kg})$ vertical load and $2000($ rpm $)<\omega<20000($ rpm $)$ rotational speed

are negligible for the entire $\mathbf{R e}$ range.

Finally, Fig. 4.22 depicts the variation of the $\log$ decrement versus Reynolds number for the $400(K g)$ rigid rotor $(200(K g)$ load per bearing). The relatively light weight of the shaft along with the high cross-coupled stiffness values yields the instability of the bearing for most of its operating range. Beyond the general instability predictions of the bearing, it can be observed that for low $\mathbf{R e}$, convective inertia tends to increase instability by lowering the $\delta$ value. However, for higher Re values, $\delta$ is higher when convective inertia is included, and the effect of convective inertia is also larger at higher Re. The added mass has destabilizing effects on the bearing operation for the entire Re range, although, the effect is very mild as expected for oil bearings. 
Table 4.3: Water Bearing

\begin{tabular}{|c|c|}
\hline Journal Diameter $(\mathrm{mm})$ & 274.9 \\
\hline Axial Length $(\mathrm{mm})$ & 233.68 \\
\hline Radial clearance $(\mathrm{mm})$ & 0.24892 \\
\hline Pad Arc Length $(\mathrm{deg})$ & 360 \\
\hline Lubricant Density $\left(\mathrm{kg} / \mathrm{m}^{3}\right)$ & 981.057 \\
\hline Lubricant Viscosity $(\mathrm{Pa}-\mathrm{s})$ & $4.423 \mathrm{e}-4$ \\
\hline Supply pressure $(\mathrm{MPa})$ & 0,15 \\
\hline Shaft Speed $(\mathrm{rpm})$ & $500-20000$ \\
\hline Rigid rotor Mass $(\mathrm{kg})$ & 1500 \\
\hline
\end{tabular}

\subsubsection{Water Bearing}

In this section, a water sleeve bearing, depicted in Fig. (4.3), is considered. This type of bearing is used in subsea applications. However, due to proprietary policies of the manufacturers, no details of the designs or experimental data are available in public domain. The bearing design parameters listed in Table (4.3) represent a generic bearing design and DOES NOT represent any particular OEM bearing design. Since these bearings often operate under water, the ambient pressure, which serves as the supply pressure, can be very high. One side effect of operating in high ambient pressure is the absence of cavitation in the bearing and an associated $2 \pi$ film around the journal, which is notorious for creating large cross-coupled stiffness properties and corresponding stability issues [94]. The bearing studied in this section is assumed to operate in a range

$$
500(\text { rpm })<\omega<12000(\text { rpm })
$$


which corresponds to

$$
\begin{aligned}
2065.88<\boldsymbol{R e} & <123952.62 \\
3.74<\mathbf{R e}^{*} & <224.48 \\
0.092<\mathbf{S} & <5.51, \text { (Sommerfeld number) }
\end{aligned}
$$

Operation under these large $\mathbf{R e}^{*}$ values means that inertia effects can be very significant. In order to study the impact of this effect, a bearing with an identical geometry and zero supply pressures (at the axial ends) is used as a comparison.

Figure (4.23) depicts the steady-state behavior of this bearing. It is evident from the journal center locus plot that the convective inertia effect plays a significant role in the equilibrium position for steady state operation of the bearing. In general, convective inertia increases the load capacity of the bearing which has the effect of reducing the eccentricity ratio and bringing the equilibrium position closer to the vertical line of symmetry.

In practice, this type of water bearings operate with high supply/ambient pressure provided at the bearing's axial ends. High supply pressure suppresses cavitation and as is shown in Fig. (4.23(b)], causes the journal center to translate horizontally. The classical Reynolds equation predicts a $0^{\circ}$ attitude angle, however, by including the convective inertia effects, the resulting attitude angle is $-90^{\circ}<\phi<0^{\circ}$.

The dynamic coefficients of the water bearing are illustrated in Figs. 4.24 4.31). The traditional Reynolds equation with high supply/ambient pressure predicts zero principle stiffness both in the horizontal and vertical directions. However, inclusion of convective inertia in the analysis results in significant principle stiffnesses (refer to Fig. (4.24)). On the other hand, the large cross-coupled stiffnesses predicted for high pressure water bearings are indifferent to the convective inertia effects (Fig. 4.25). 
This trend also holds for the cross-coupled stiffnesses of water bearings with zero ambient pressure. In Fig. 4.26), considerable direct damping can be observed and the values increase with rotational speed and $\mathbf{R e}$. The principle damping coefficients predicted for high supply pressure in general are larger than those of the zero supply pressure bearing, and convective inertia has a negligible effect on principle damping. In contrast, the cross-coupled damping terms are effectively zero and have lower values for high supply pressure water bearings compared with the values of the zero supply pressure water bearing. For all cases, i.e., zero and high supply pressures, the cross-coupled damping coefficients are negligible compared to the principle terms.

In Figs. 4.28 4.29, the principle added mass coefficients are illustrated versus Re. These plots report huge principle added mass values for both $M_{x x}$ and $M_{y y}$. The values of these coefficients are approximately $30 \%$ to $90 \%$ of the shaft mass for $P=0$ and $P=15(\mathrm{MPa})$ supply pressure water bearings, respectively. The inclusion of the convective inertia terms has minor effects on the principle mass coefficients, while the secondary inertia term alters these coefficients dramatically. In general, it can be seen that by including the secondary temporal inertia term, the trend with respect to $\mathbf{R e}$ is altered from descending to ascending for low $\mathbf{R e}$ values, while its effect vanishes for high rotational speeds and Re. Note that this effect can be so dominant that it results in significant negative principle added mass coefficients. Cross coupled added mass coefficients in general approach zero for high rotational speeds, while for low rotational speeds they can be considerable, see Figs. 4.30 \& 4.31). The absolute values of these coefficients without the secondary inertia effect are negligible, however, their inclusion can result in large negative values, especially for low rotational speeds.

Figure 4.32 illustrates the variation of $\delta$ versus rotational speed for this water bearing design. For a large extent of the operating speed domain, the bearing is unstable, which is an indication of an inadequate design. However, regardless of the 
bearing instability, general trends can still be observed. First, it can be seen that the convective inertia has stabilizing effects, while the added mass effect acts to destabilize the bearing. This is illustrated by the fact that the cases which include the convective inertia but no added mass effect exhibit the highest log decrement values. Finally, it can be seen from the results depicted in Fig. 4.32 that the secondary inertia term has a very negligible effect on the stability of the bearing. 


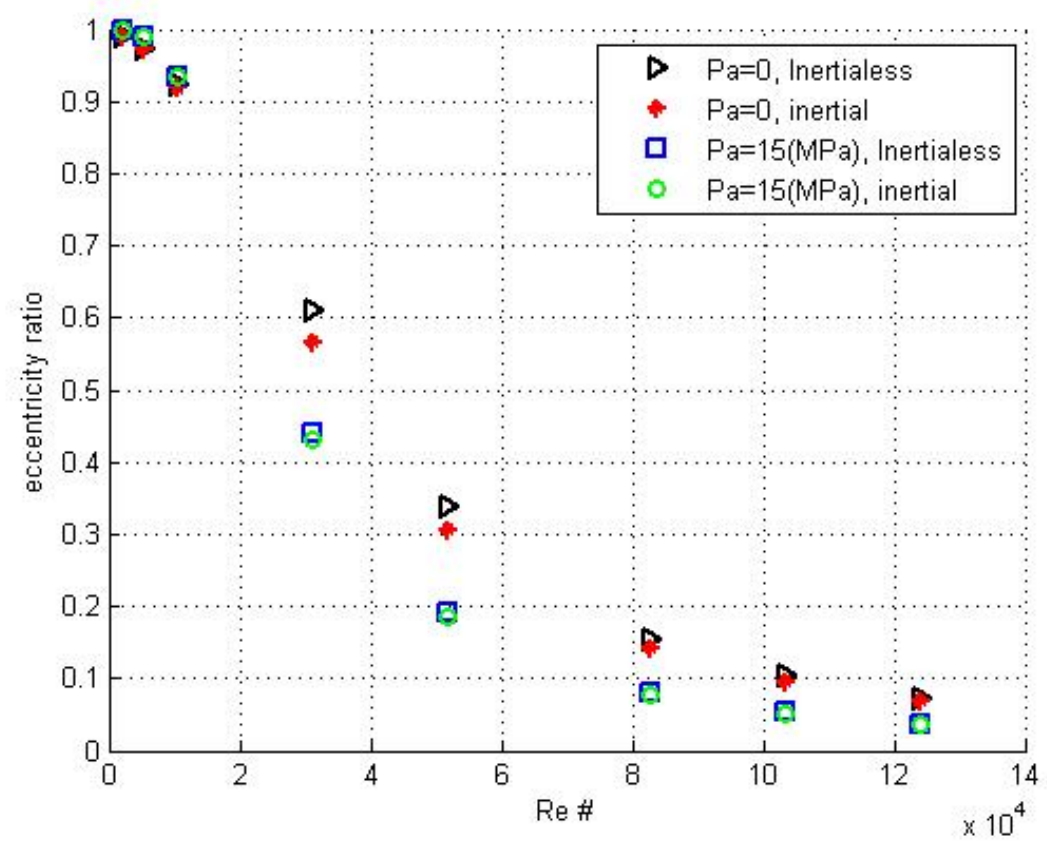

(a) eccentricity ratio versus $\mathbf{R e}$

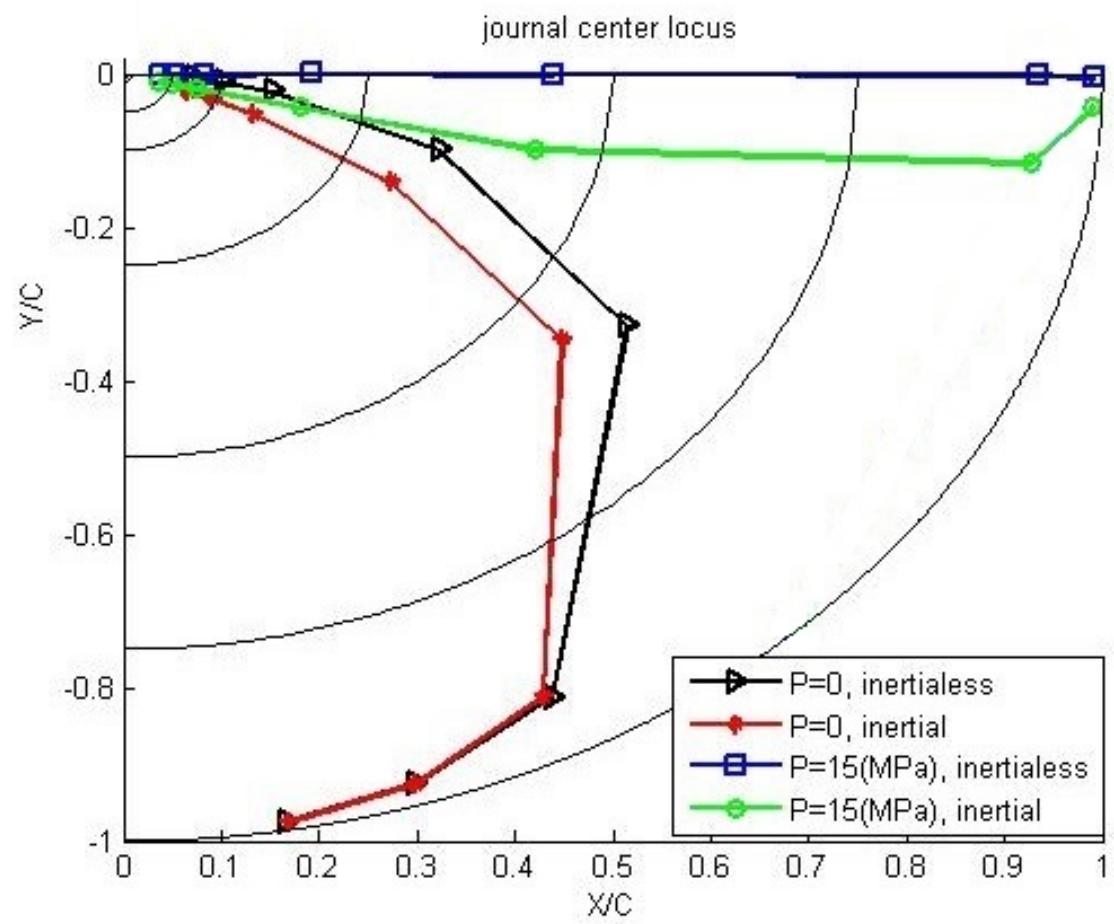

(b) journal locus

Figure 4.23: Steady state operation of water bearing with a $1500(\mathrm{Kg})$ vertical load and $500<\omega<12000($ rpm $)$ operational speed range 


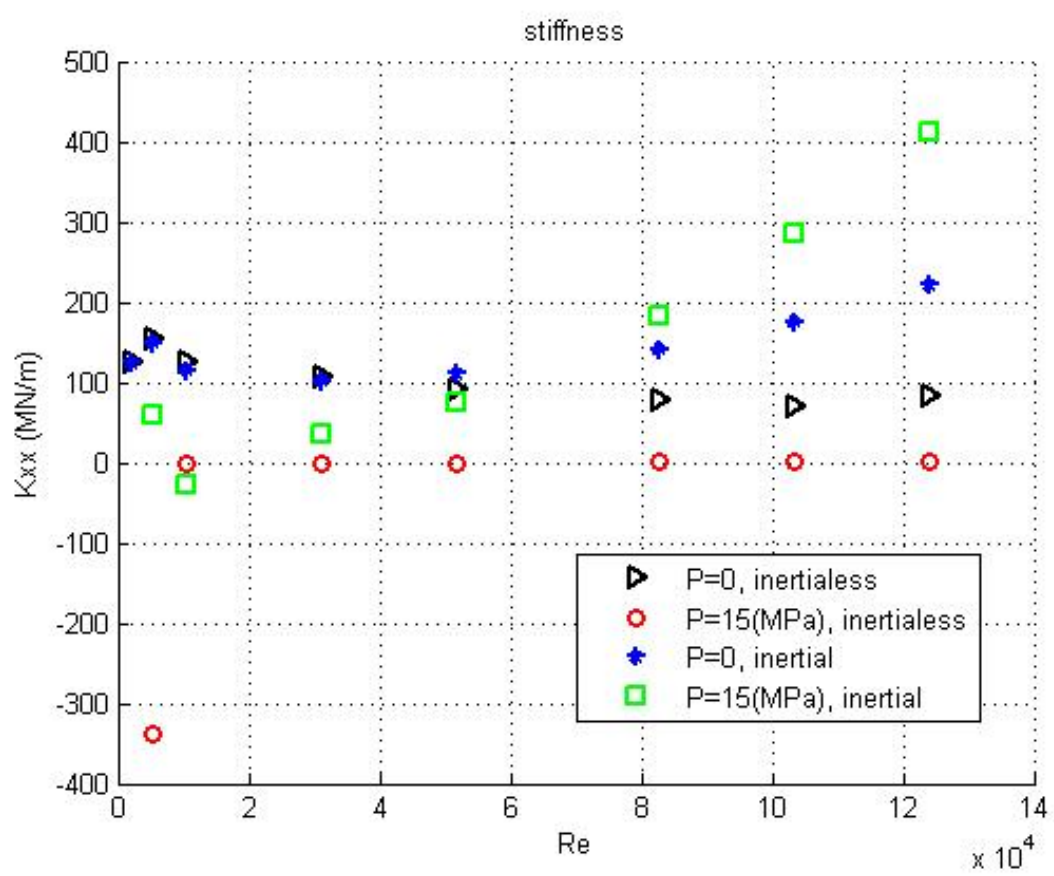

(a) $\mathrm{Kxx}$

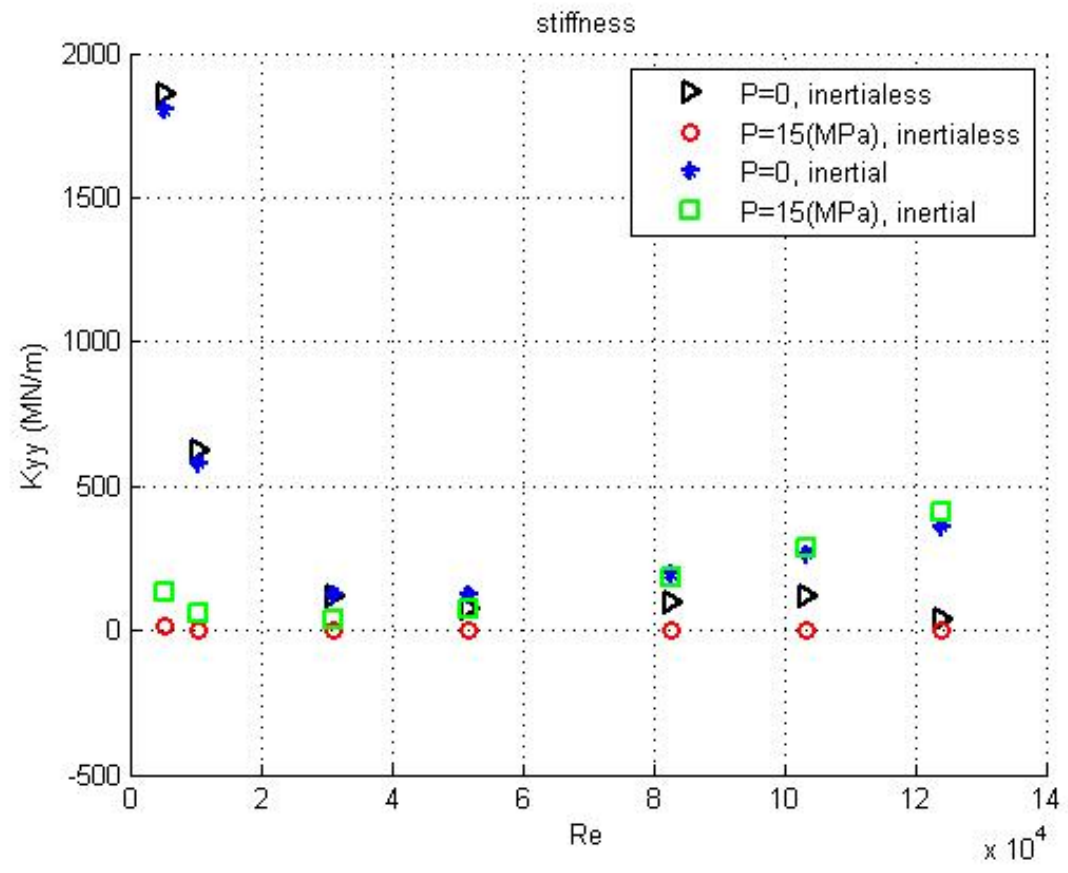

(b) Kyy

Figure 4.24: Principle stiffness, water bearing with $1500(\mathrm{Kg})$ vertical load and $500<\omega<12000($ rpm $)$ operational speed range 


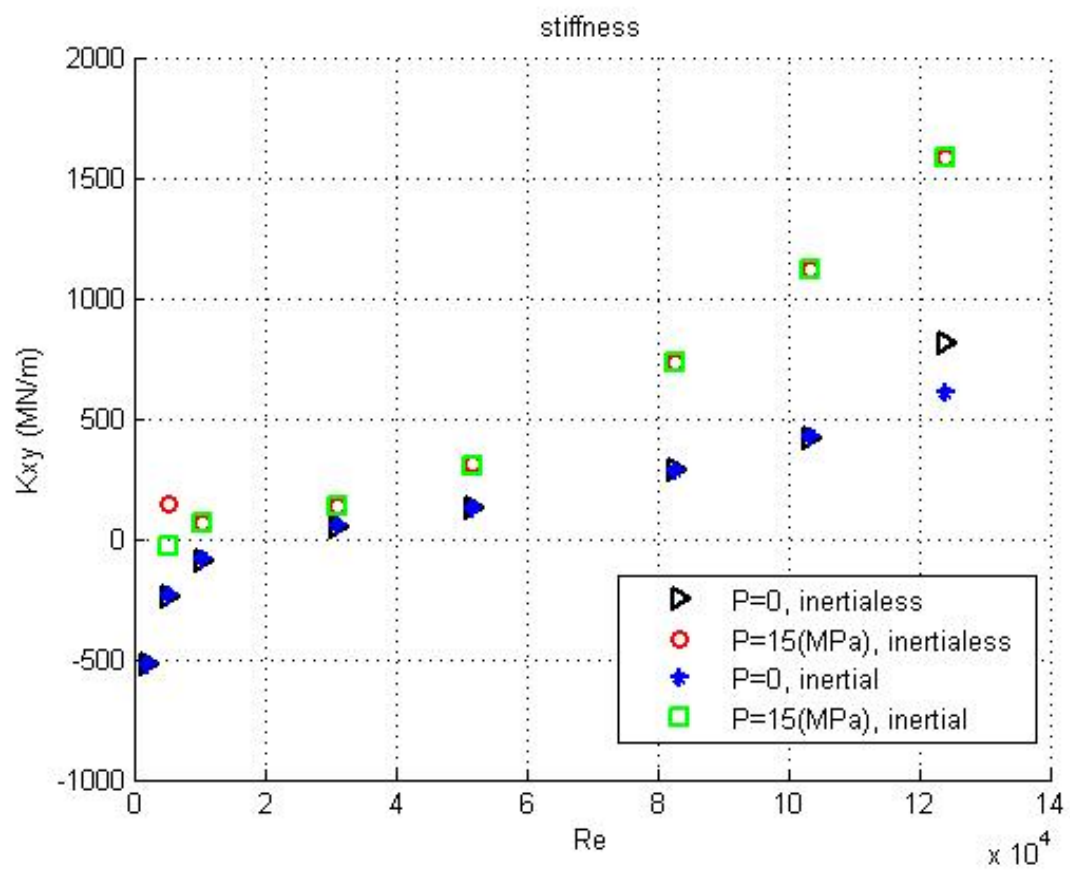

(a) Kxy

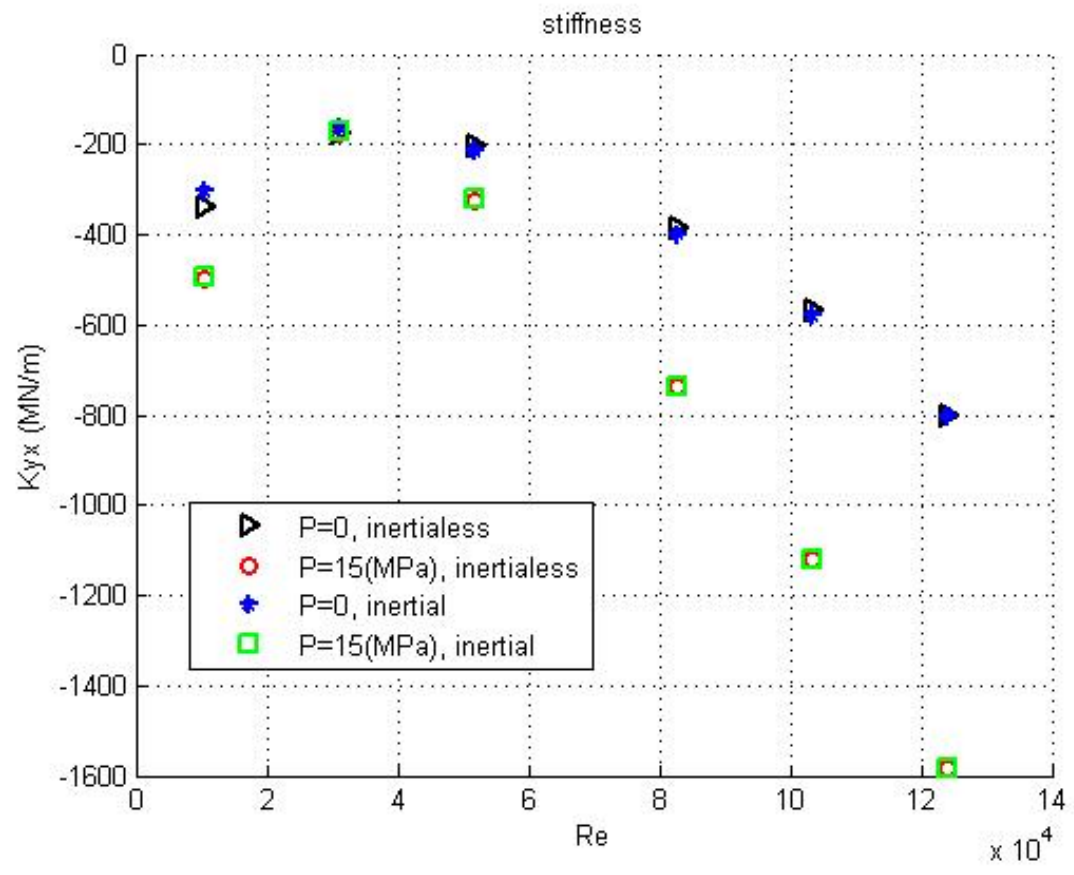

(b) Kyx

Figure 4.25: Cross-coupled stiffness, water bearing with $1500(\mathrm{Kg})$ vertical load and $500<\omega<12000($ rpm $)$ operational speed range 


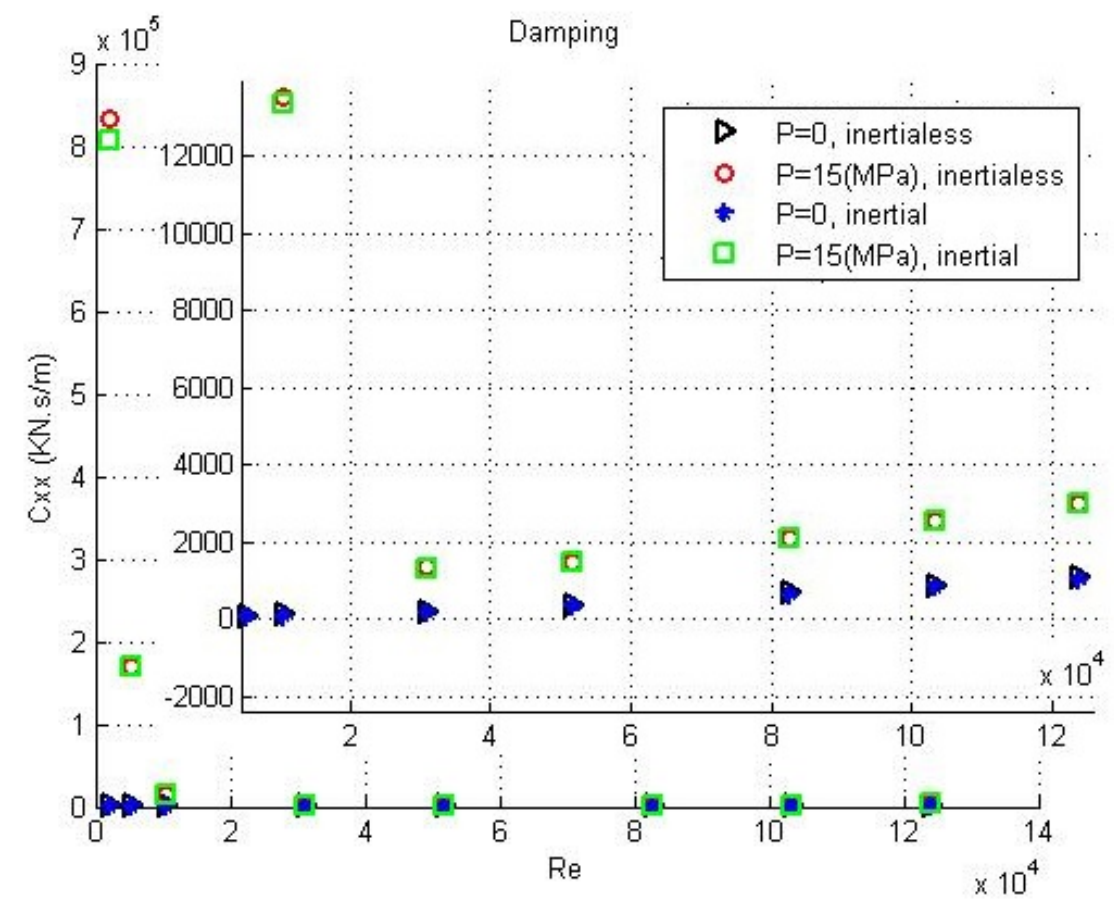

(a) $\operatorname{Cxx}$

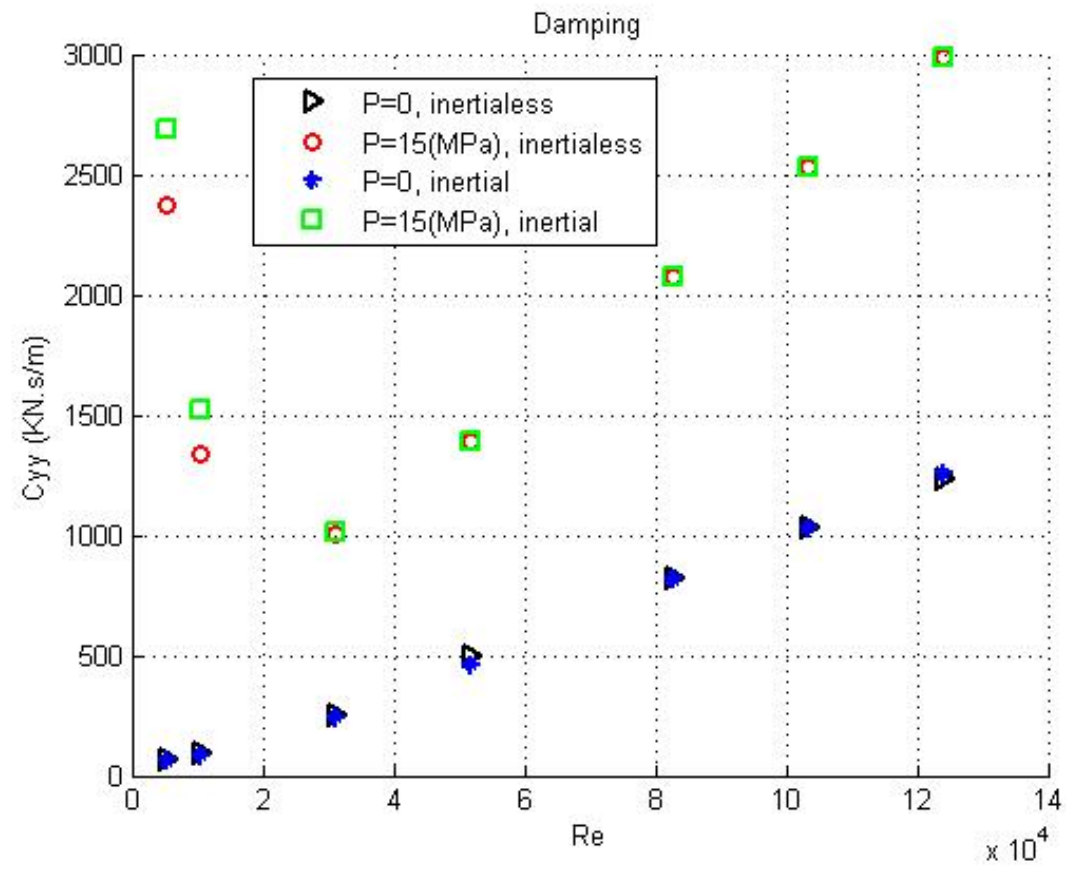

(b) Cyy

Figure 4.26: Principle damping, water bearing with $1500(\mathrm{Kg})$ vertical load and $500<\omega<12000(\mathrm{rpm})$ operational speed range 


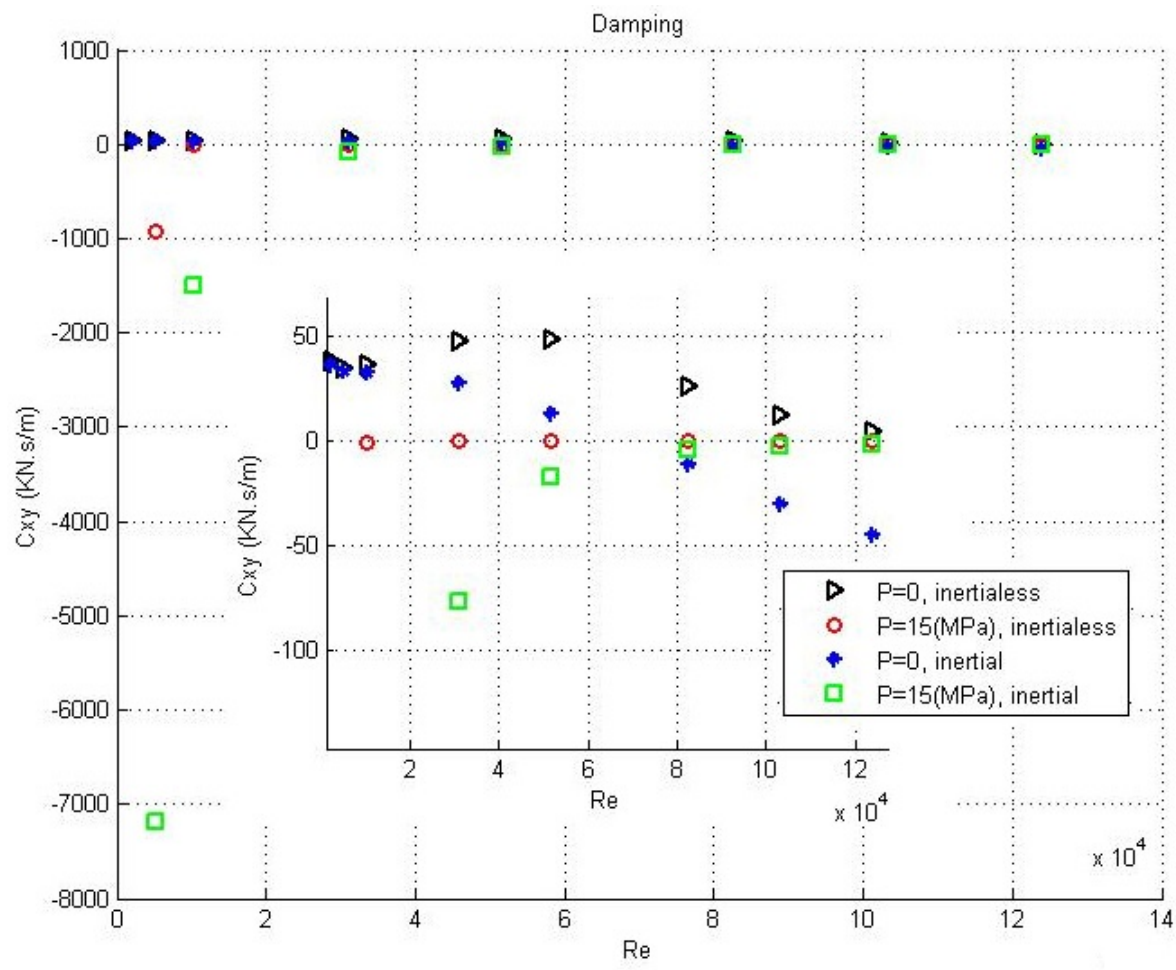

(a) Cxy

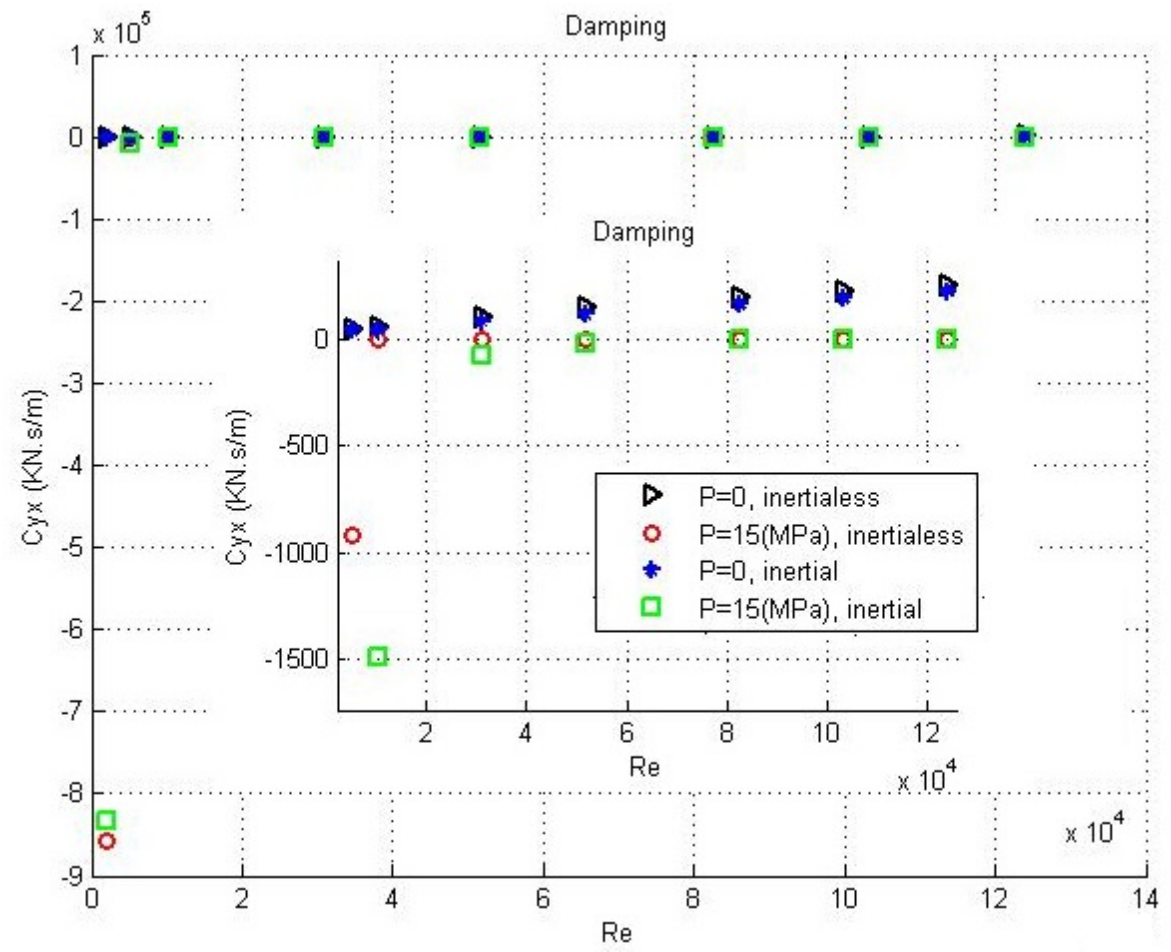

(b) Cyx

Figure 4.27: Cross-coupled damping, water bearing with $1500(\mathrm{Kg})$ vertical load and $500<\omega<12000(\mathrm{rpm})$ operational speed range 


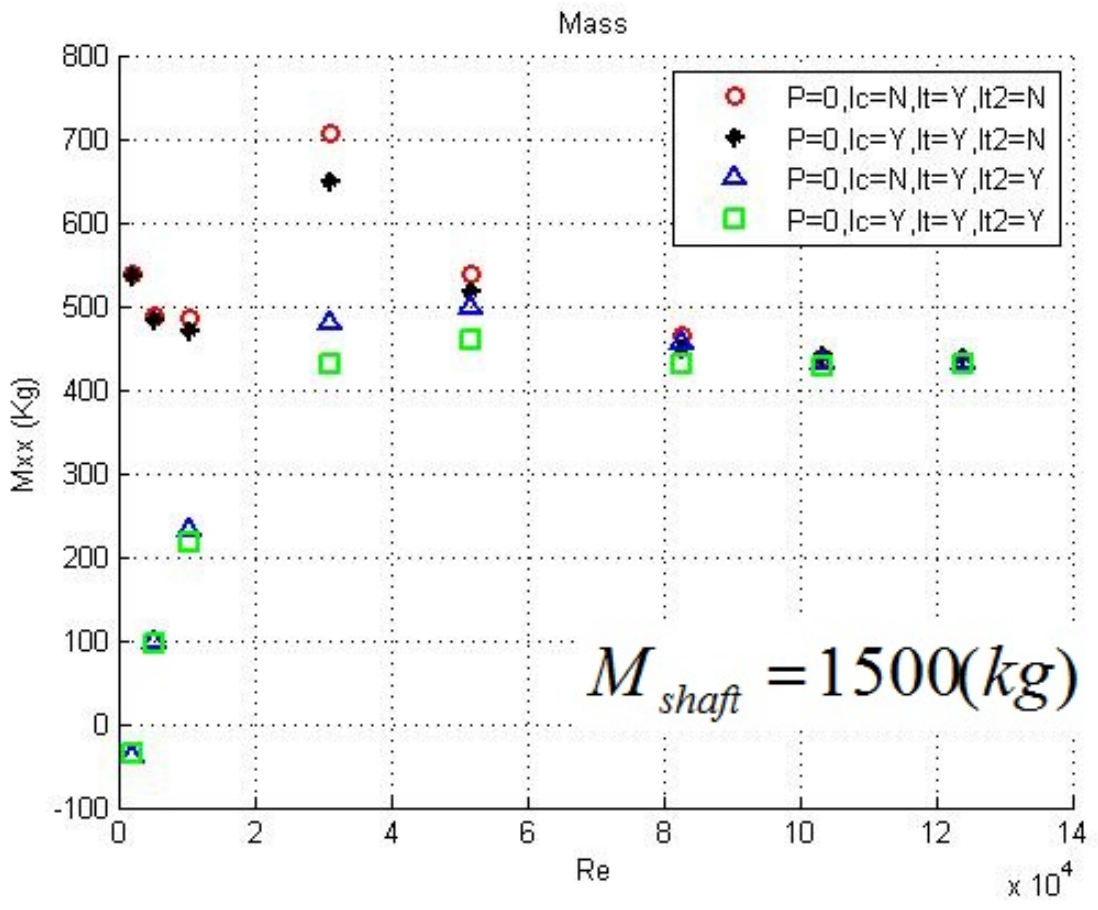

(a) Mxx, $P_{s}=0$

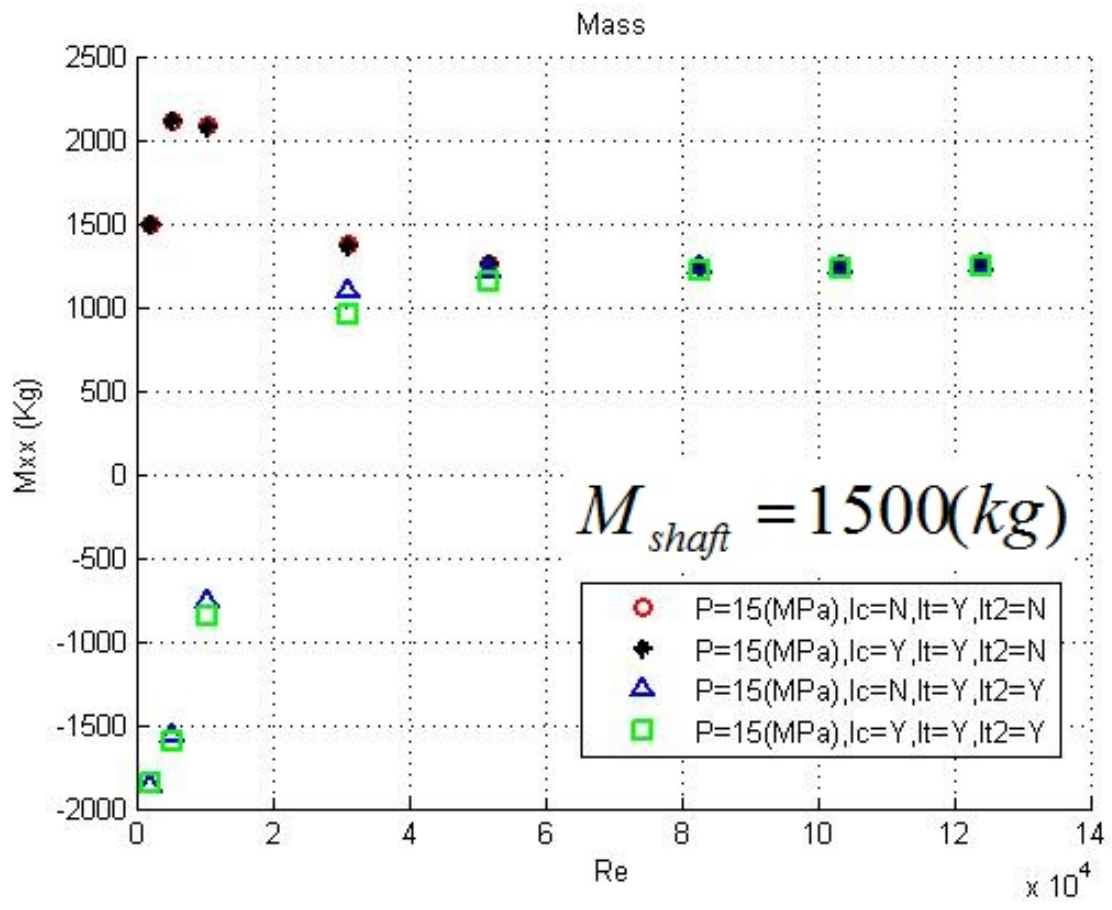

(b) $\mathrm{Mxx}, P_{s}=15(M P a)$

Figure 4.28: Horizontal principle added mass, water bearing with $1500(\mathrm{Kg})$ vertical load and $500<\omega<12000(\mathrm{rpm})$ operational speed range 


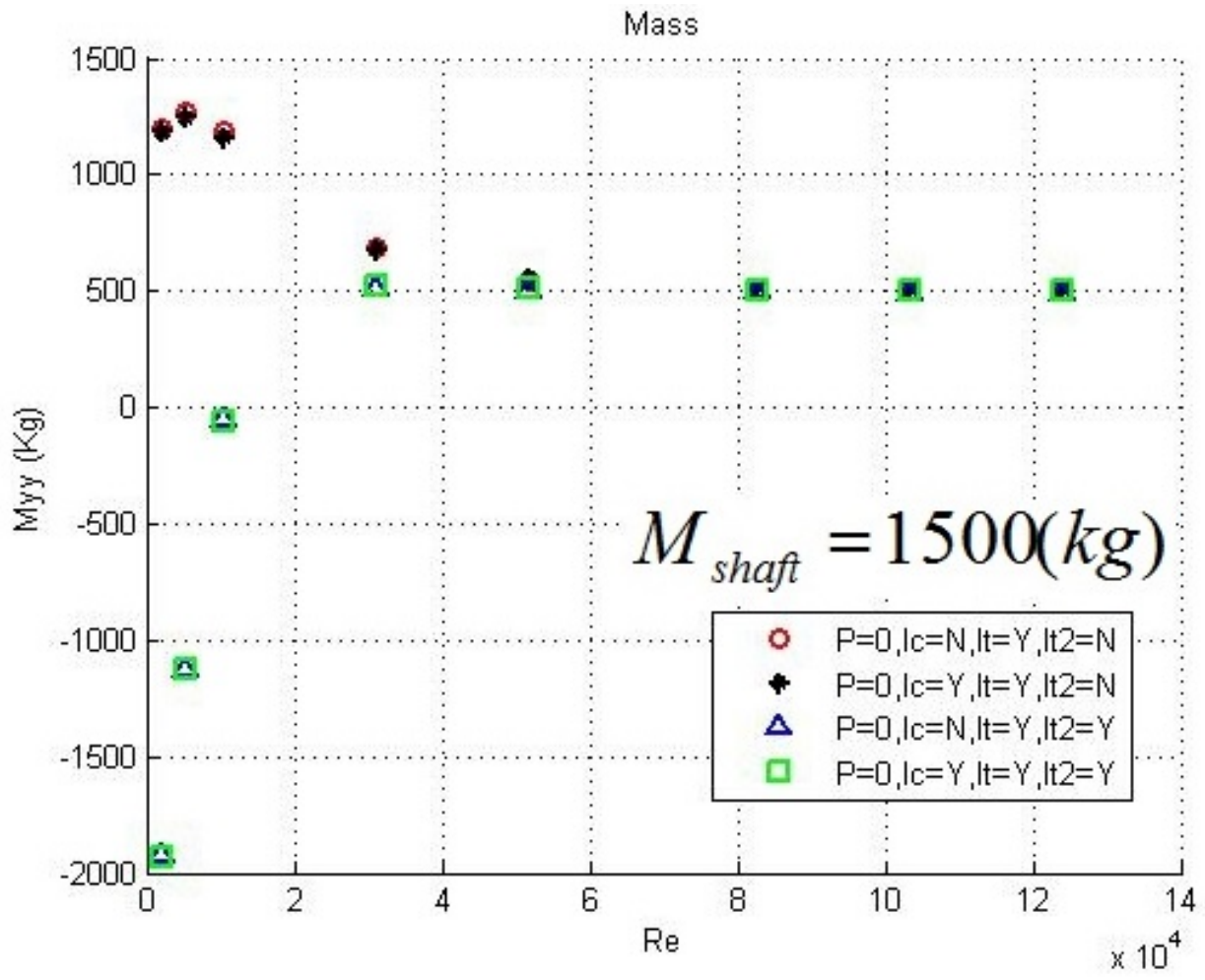

(a) Myy, $P_{s}=0$

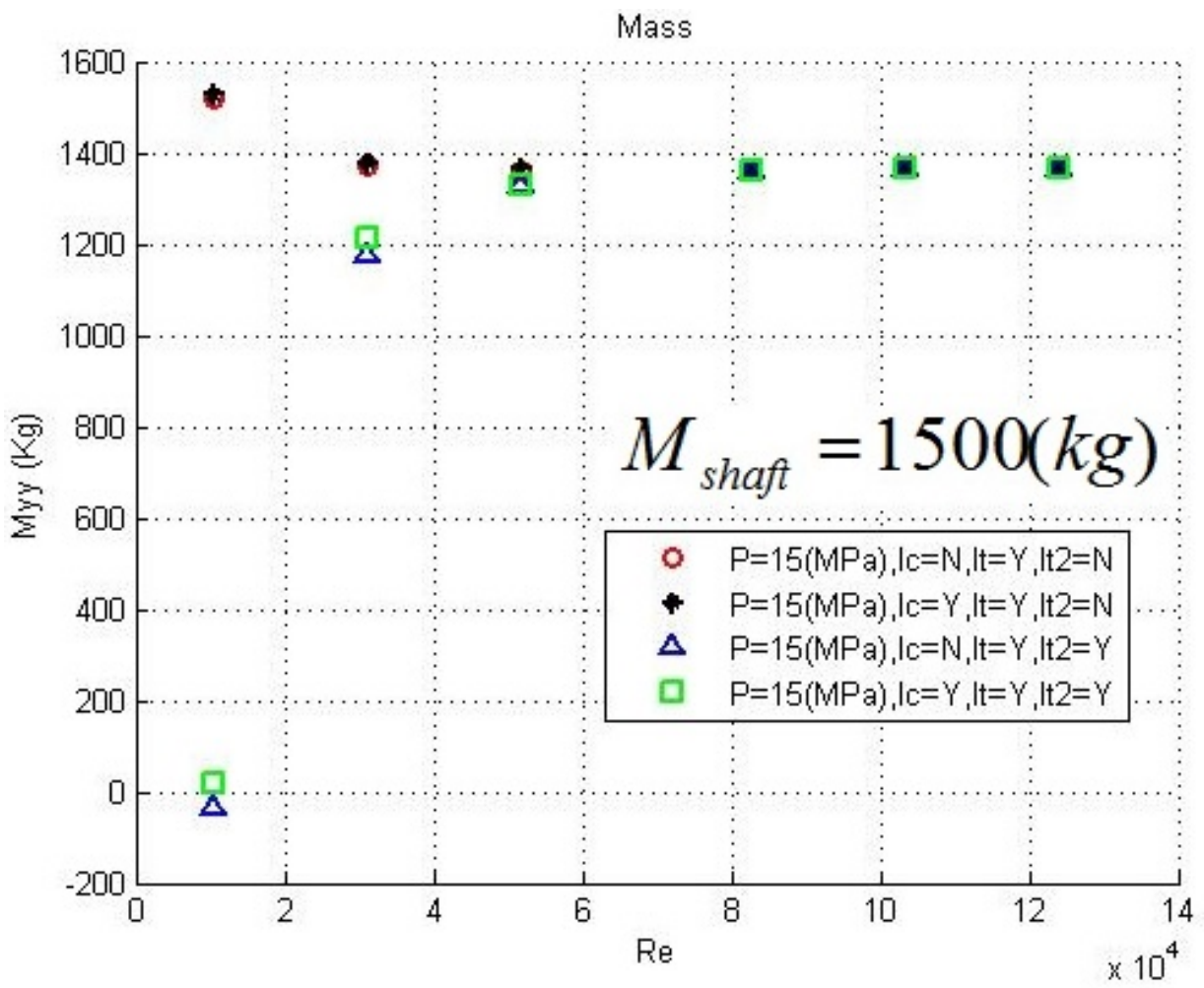

(b) Myy, $P_{s}=15(M P a)$

Figure 4.29: Vertical principle added mass, water bearing with $1500(\mathrm{Kg})$ vertical load and $500<\omega<12000$ (rpm) operational speed range 


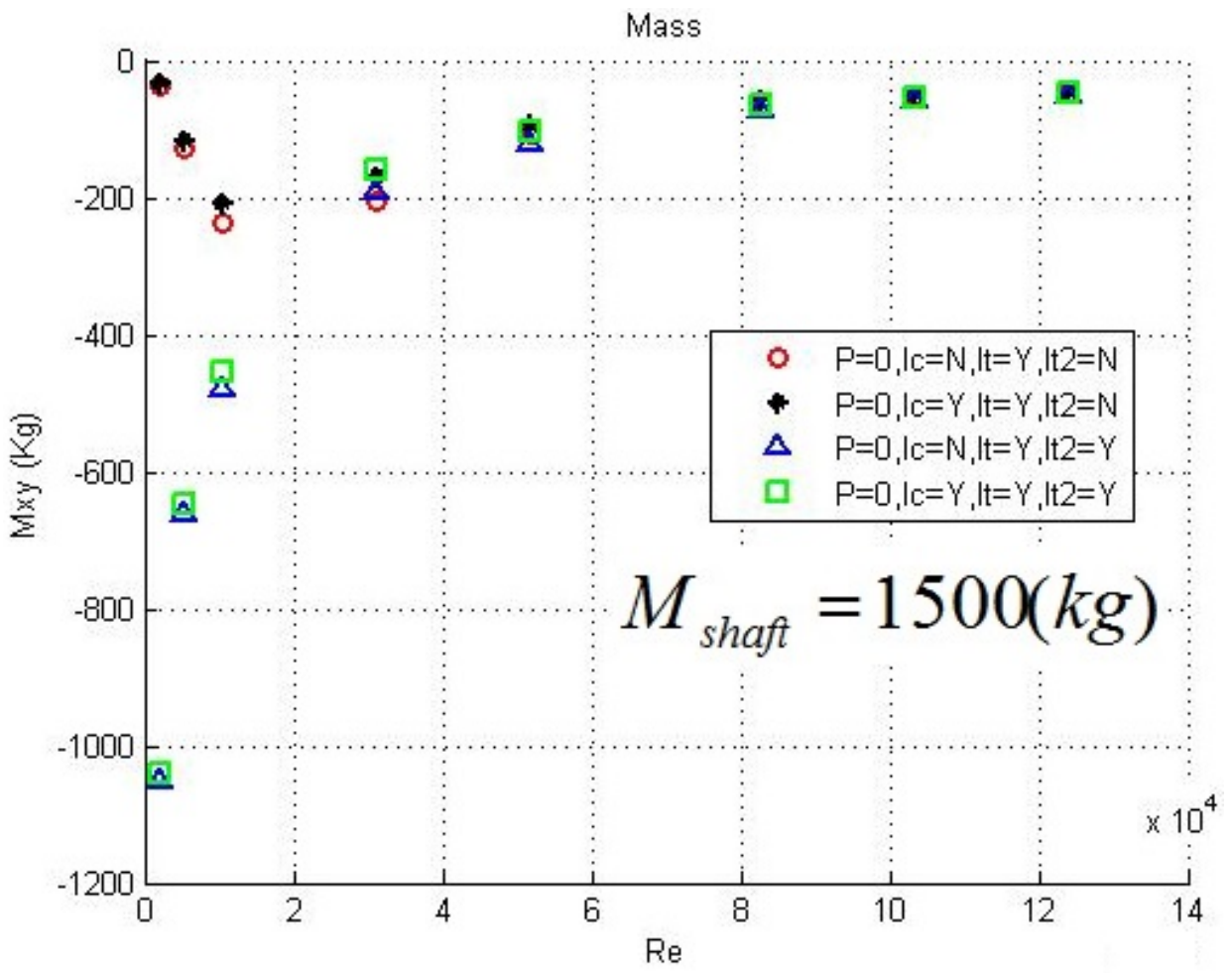

(a) Mxy, $P_{s}=0$

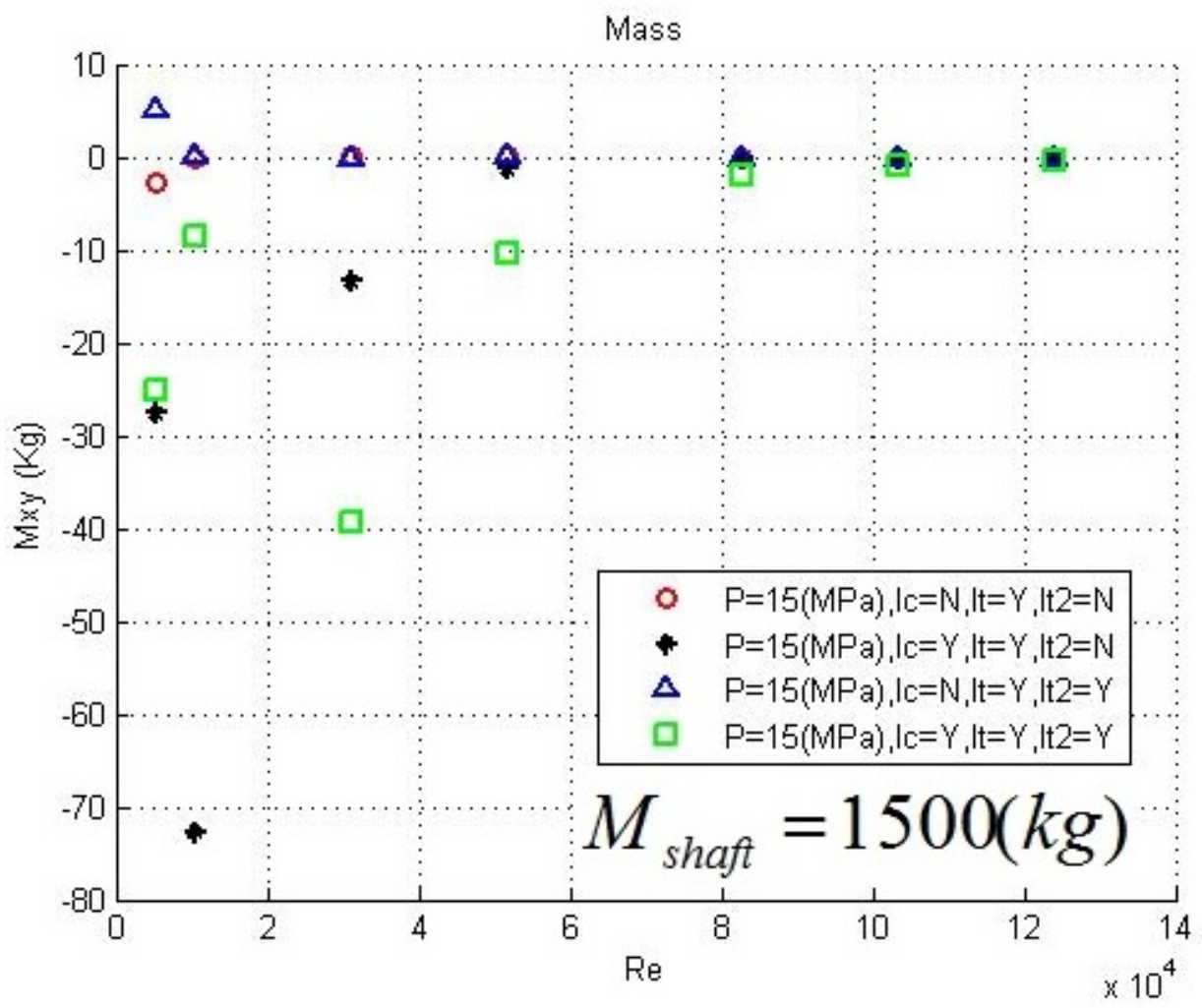

(b) Mxy, $P_{s}=15(M P a)$

Figure 4.30: Cross-coupled added mass, water bearing with $1500(\mathrm{Kg})$ vertical load and $500<\omega<12000$ (rpm) operational speed range 


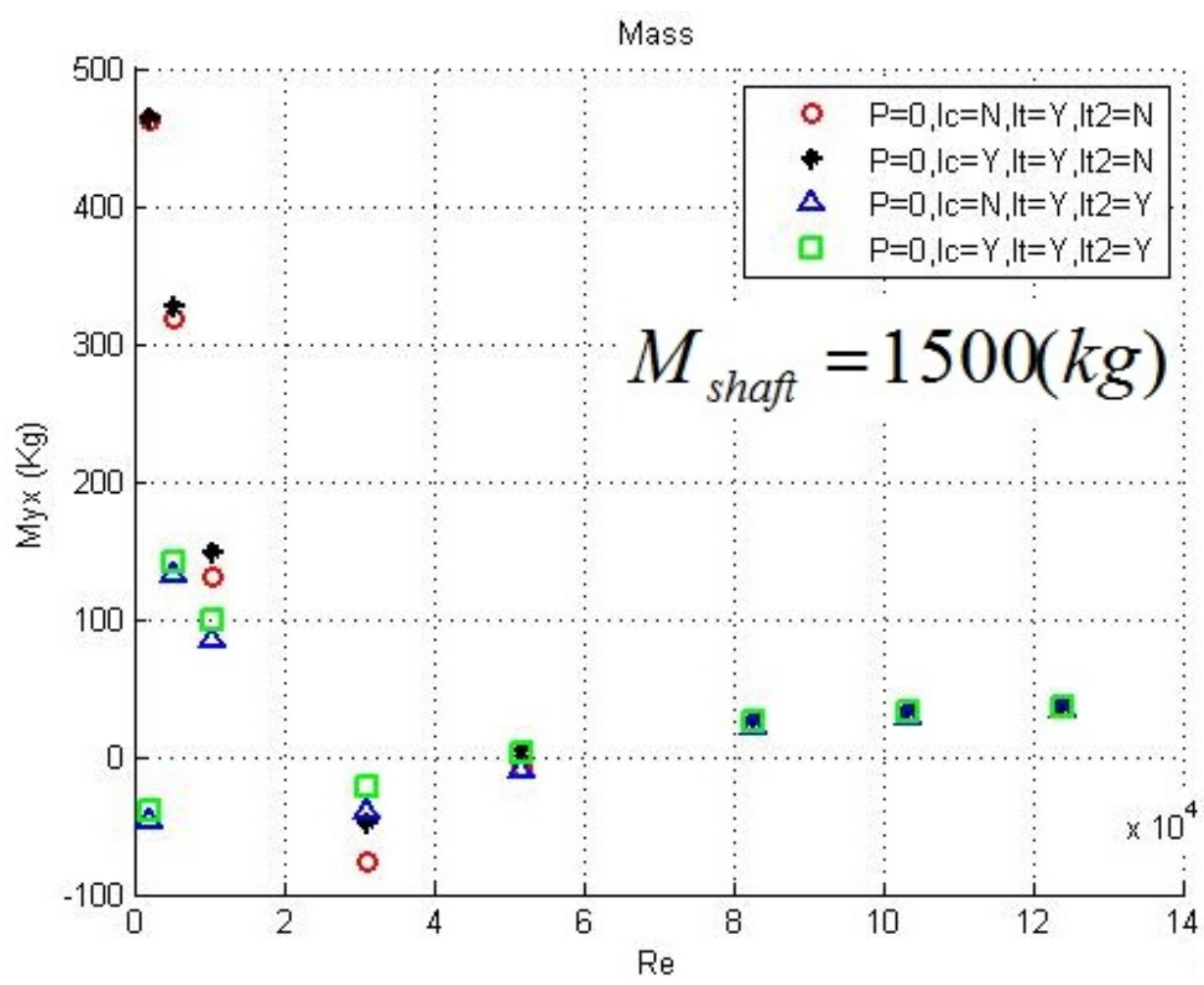

(a) Myx, $P_{s}=0$

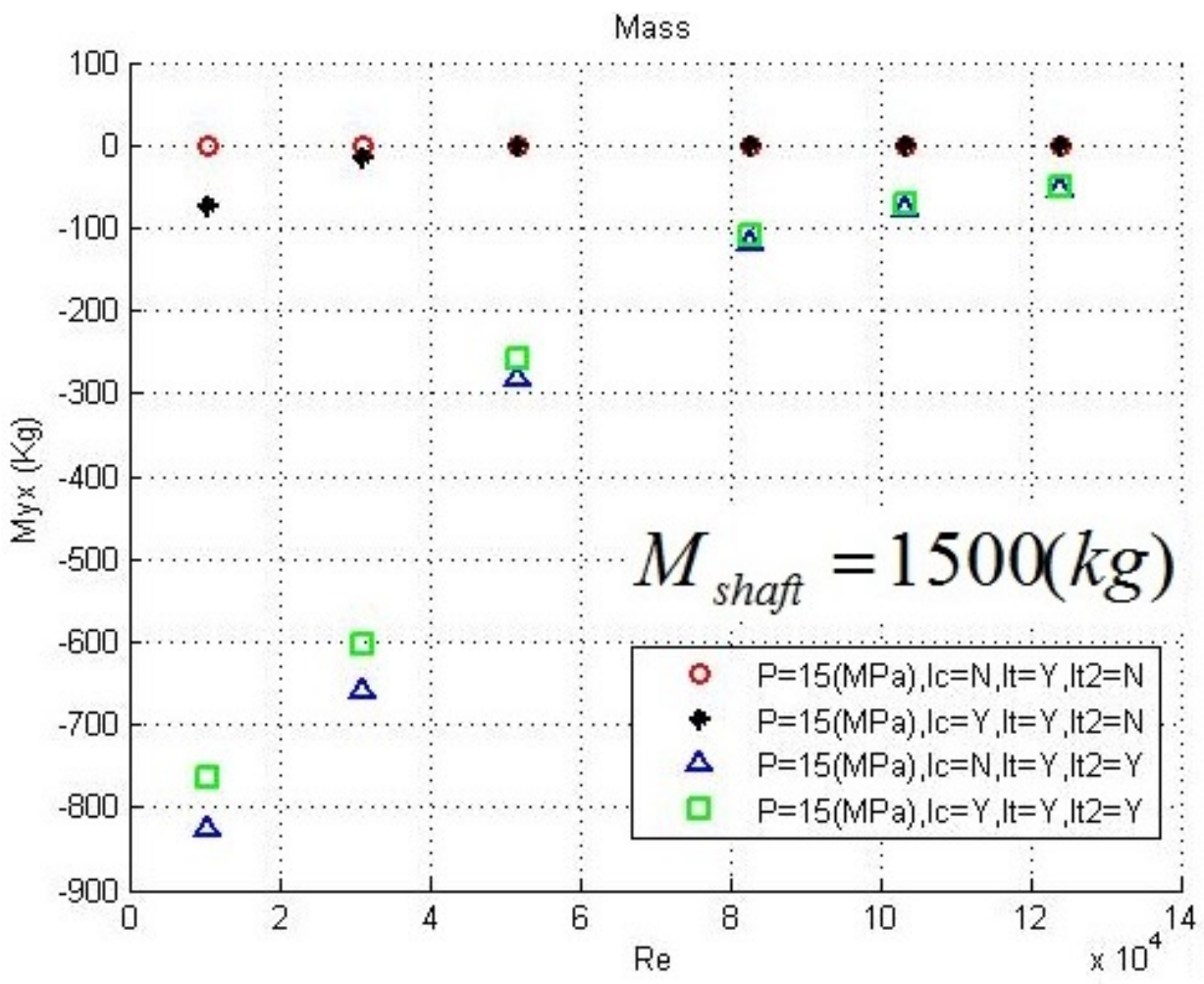

(b) $\mathrm{Myx}, P_{s}=15(M P a)$

Figure 4.31: Cross-coupled added mass, water bearing with $1500(\mathrm{Kg})$ vertical load and $500<\omega<12000$ (rpm) operational speed range 


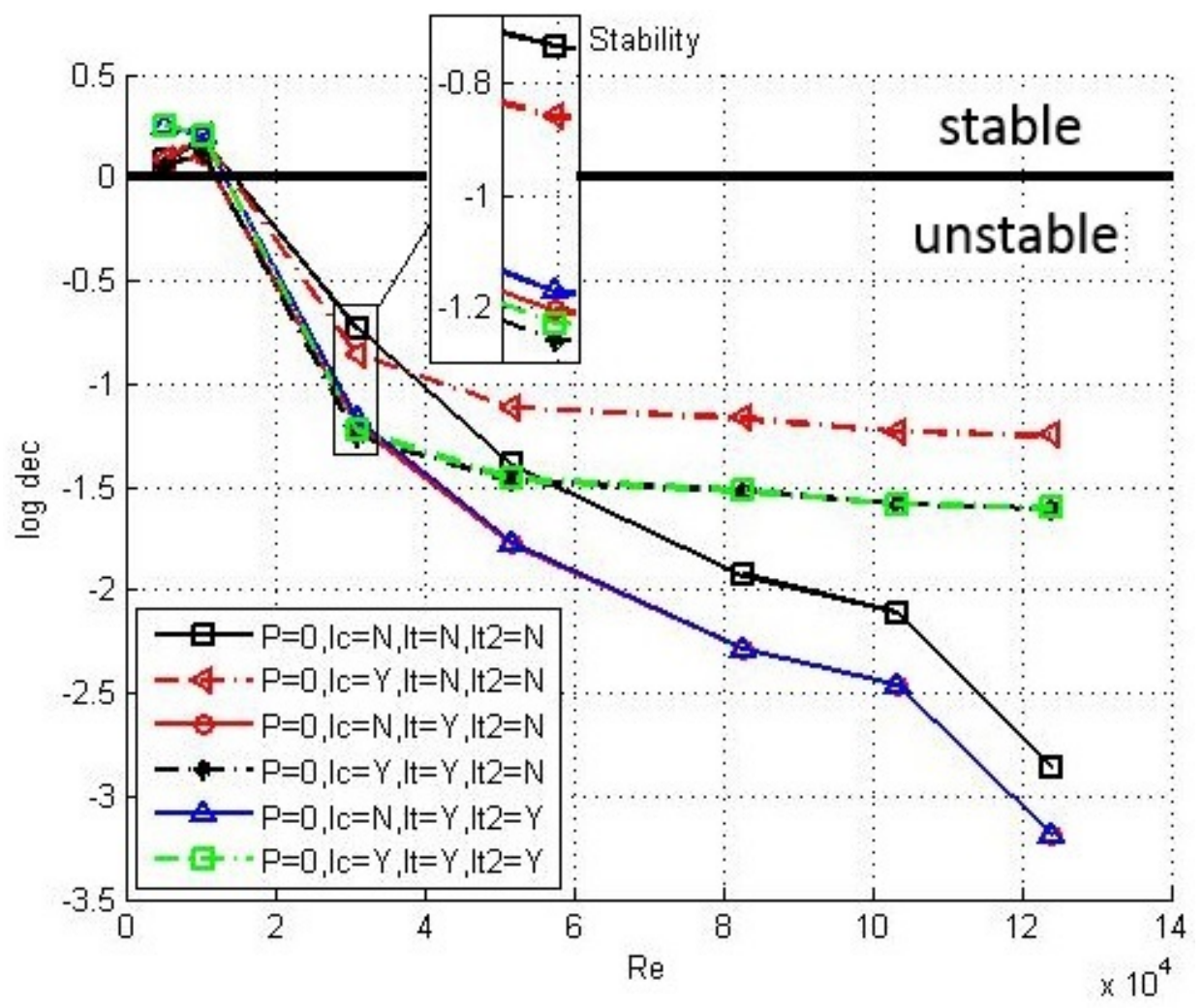

(a) $\log$ decrement, $P_{s}=0$

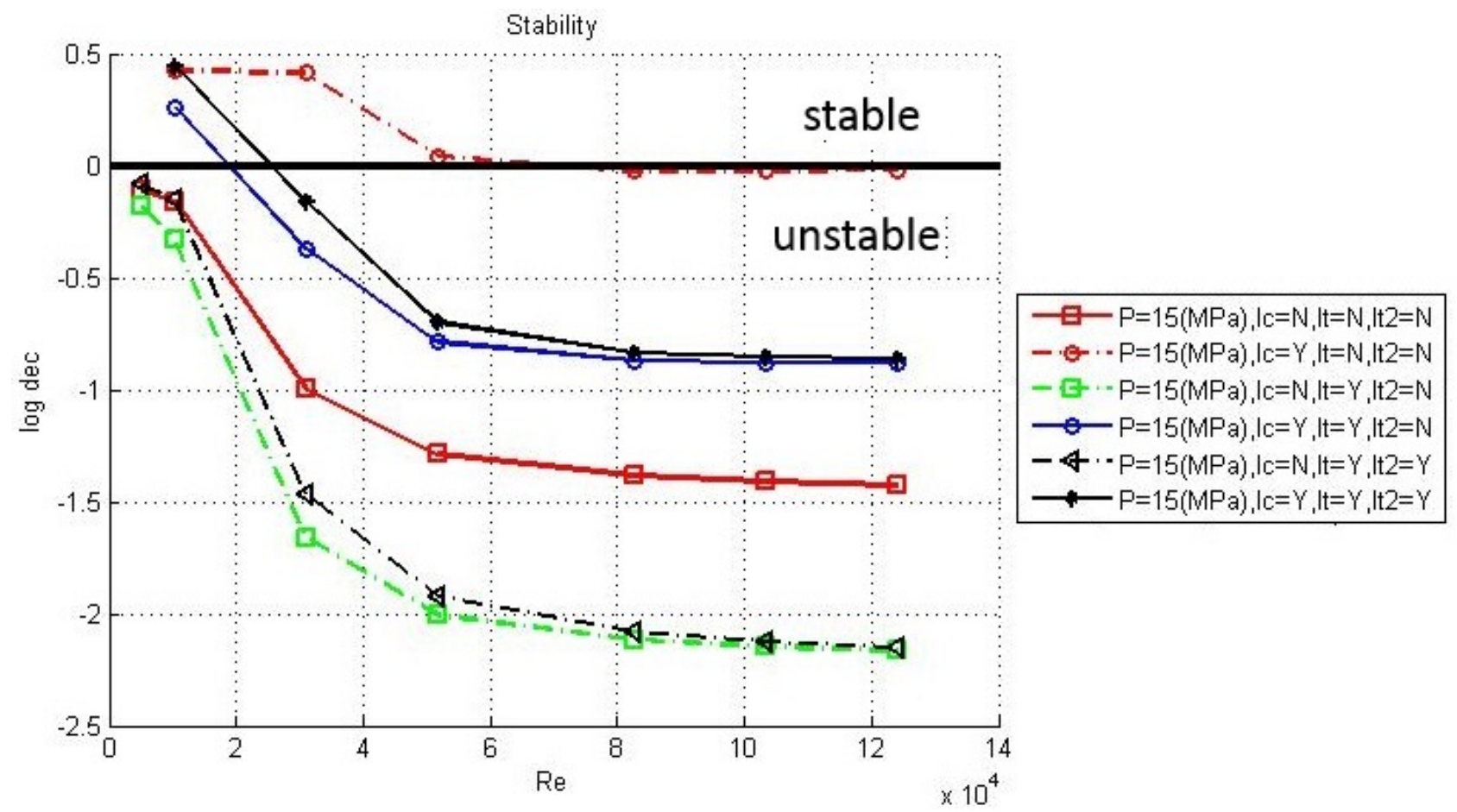

(b) $\log$ decrement, $P_{s}=15(M P a)$

Figure 4.32: Stability of a water bearing with $1500(\mathrm{Kg})$ vertical load and $500<\omega<$ $12000($ rpm $)$ operational speed range 


\section{Chapter 5}

\section{Squeeze Film Dampers}

The need to improve the stability of rotordynamic systems encouraged the use of squeeze film dampers (SFDs) as a simple means of providing extra damping. From very early on after the advent of SFDs in 1960s, simple theories attempted to capture and predict the behavior of these bearings [88, 1, 9]; however, recent findings have revealed a very complex behavior deviating away from these simple models [80, 78, 89, 95, 10, 76.

This chapter puts forth a comprehensive theory to model these bearings using an efficient and thorough approach. In Sec. (5.1), an overview of the geometry, operation, and different aspects of SFDs is presented. Section (5.2) suggests reduced versions of the extended Reynolds equation applicable to SFDs. A computer code named "MAXSFD" is developed and explained in Sec. (5.3). Four SFD configurations are modeled by MAXSFD and the results are compared and contrasted with experimental data and SQFDAMP [1], in Sec. (5.4). 


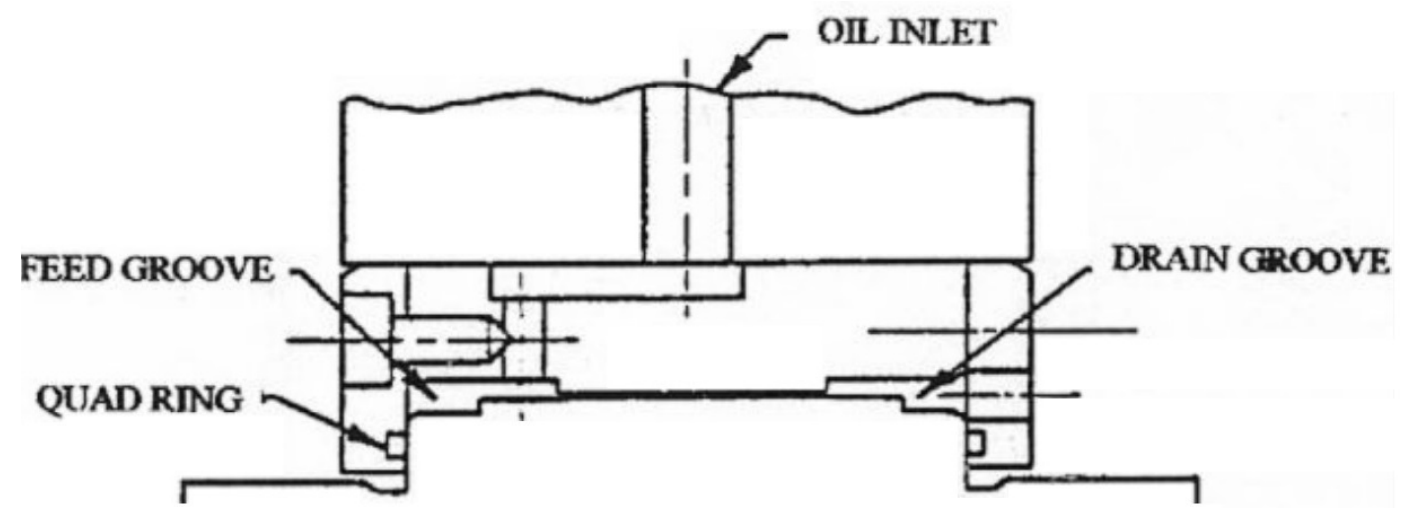

(a) side groove configuration [11.

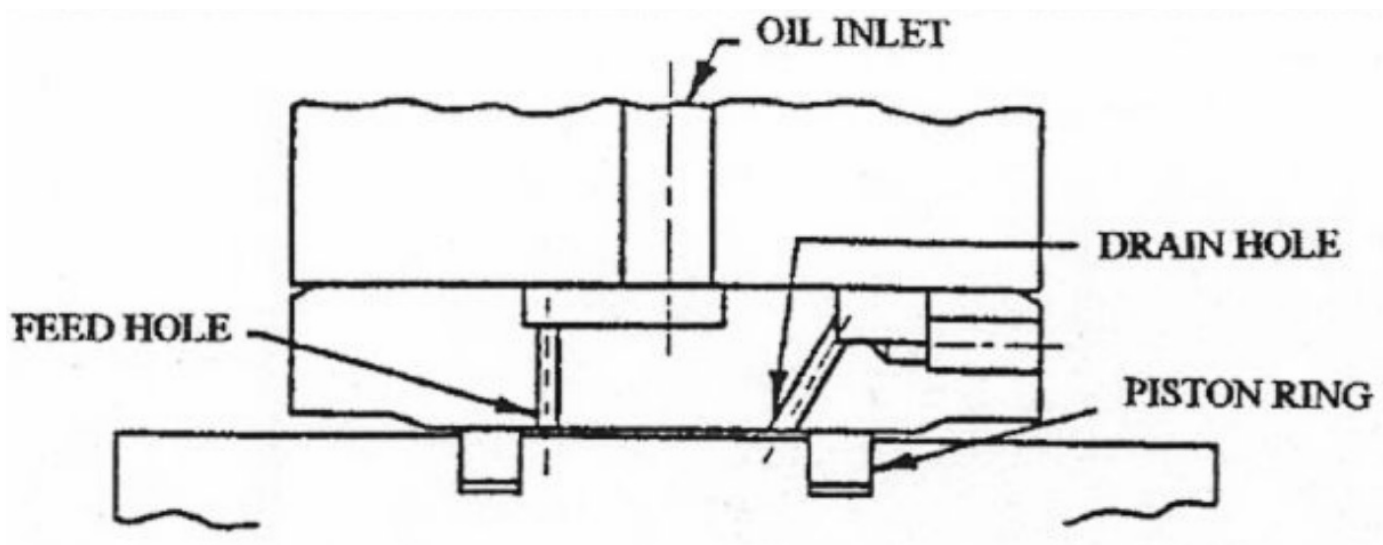

(b) no groove configuration [1]

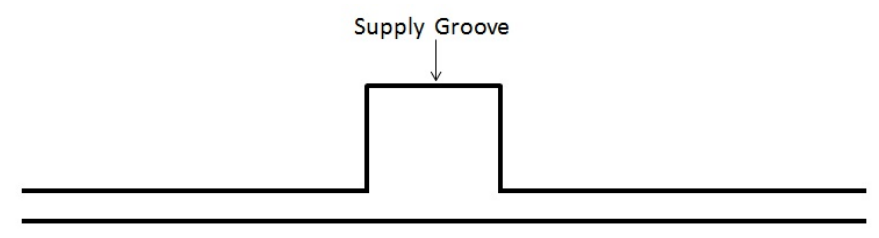

(c) central groove, open-end configuration

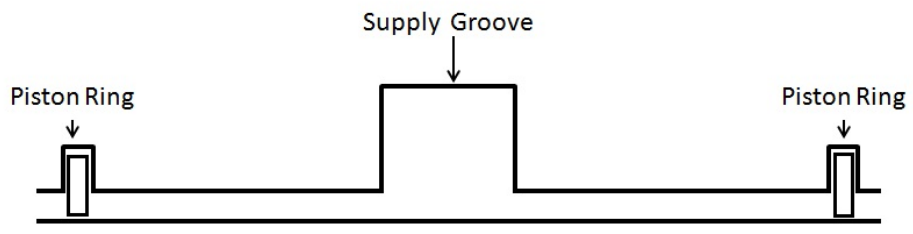

(d) central groove, sealed-end configuration

Figure 5.1: Different configurations of squeeze film dampers, axial cutaway view. 


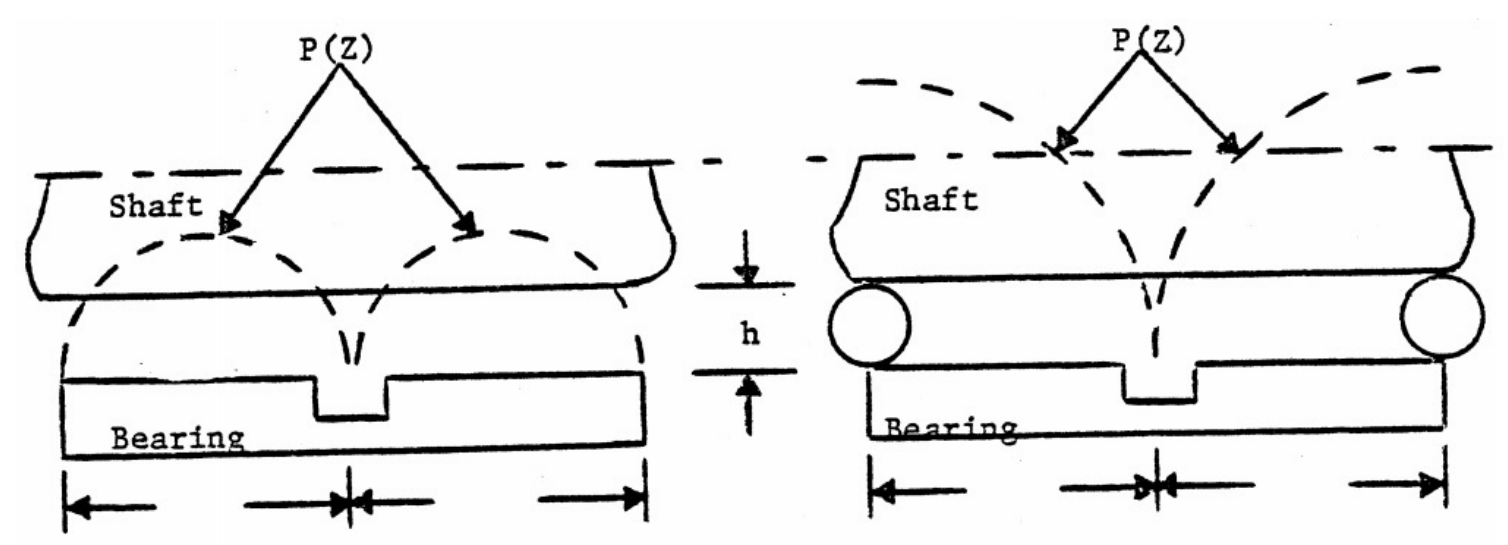

Figure 5.2: Traditional perception of central groove; zero dynamic pressure in groove [1]

\subsection{Geometry and Operation}

Squeeze film dampers come in various designs. The main feature of a SFD is its "land" region, where a large hydrodynamic pressure is generated. Often, lubricant is fed into the land area through an adjacent feeding groove filled by a number of supply holes/nozzles, Fig. (5.1(a)). Sometimes the feeding and draining holes open directly to the land region as in Fig. (5.1(b) . In some designs, the oil, once squeezed, is allowed to leak into the ambient from open axial ends, Fig. (5.1(c). And finally, in some other designs, axial leakage is minimized by use of a pair of axial seals, shown in Fig. (5.1(d)). The existence of regions with different clearances, all of which contribute to the SFD properties, requires a flexible model to accommodate different clearance values along the entire axial length. In addition, a comprehensive model should be able to incorporate the leakage through the end seals.

\subsubsection{Groove(s)}

The groove region acts as an oil reservoir with a typical depth at least an order of magnitude larger than the land clearance. This large body of lubricant, located ad- 


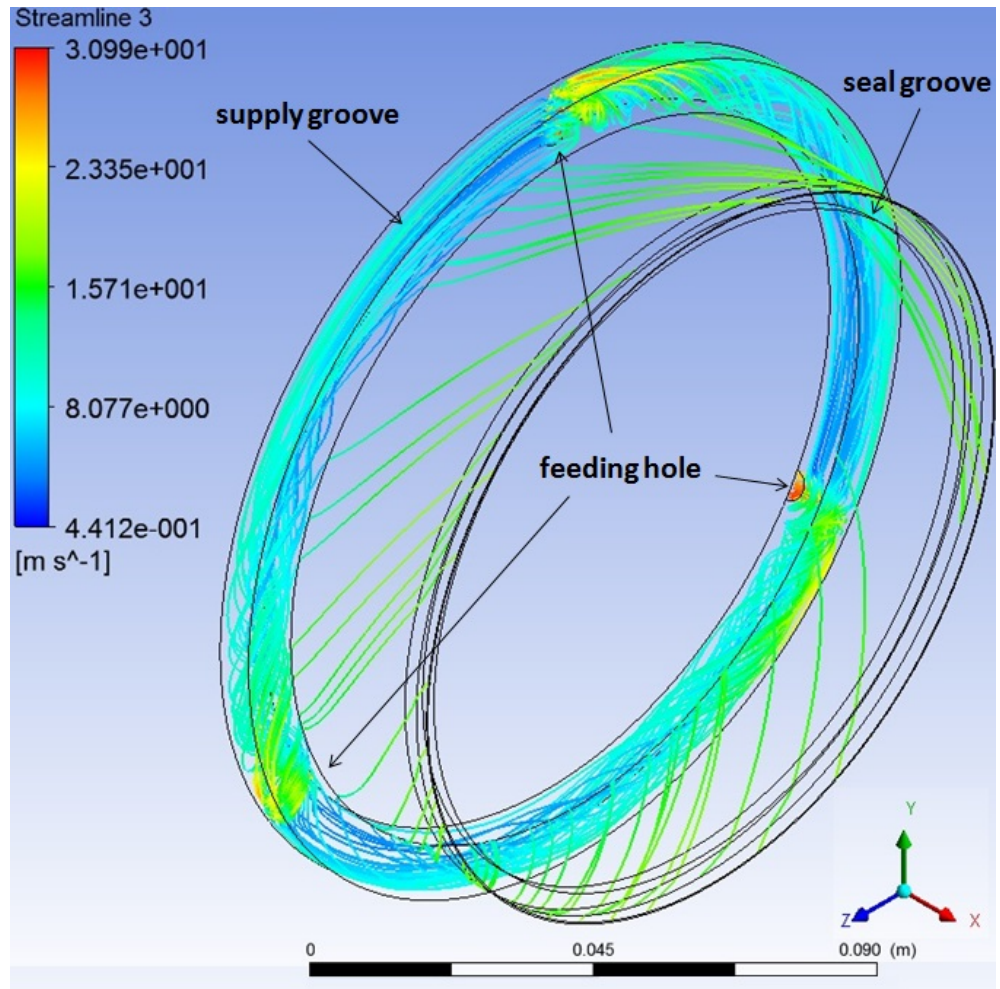

(a) 3D streamline

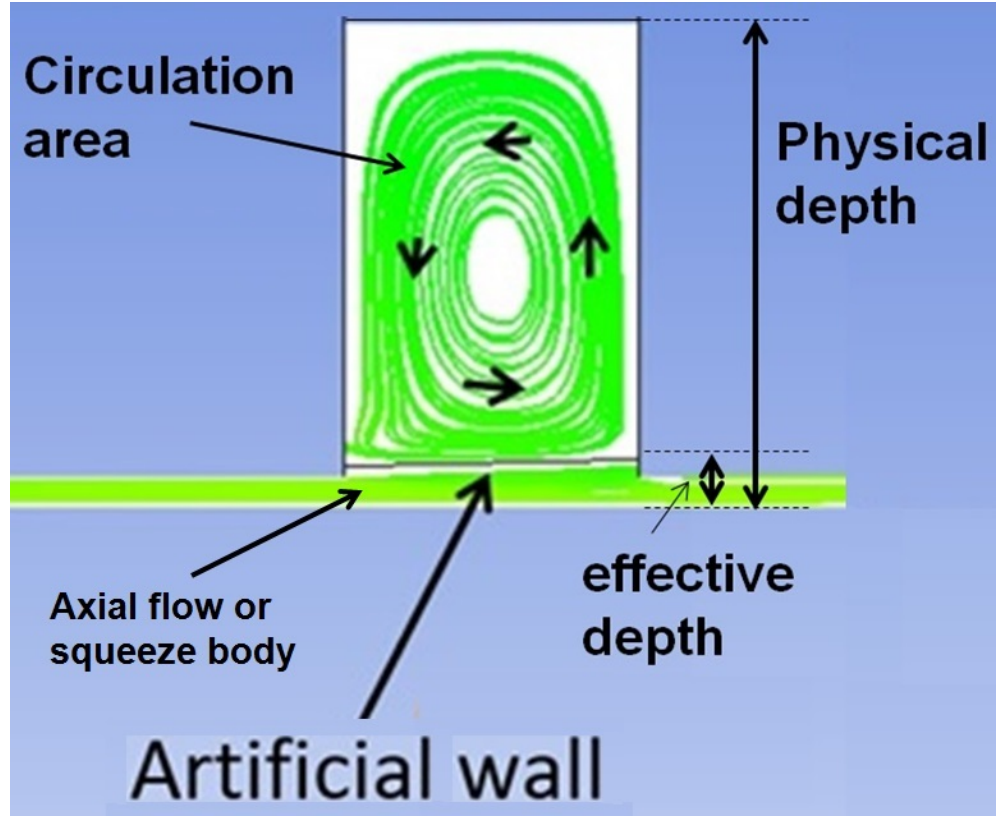

(b) circulation in groove: physical and effective depths

Figure 5.3: The streamlines of the flow in the groove regions of SFD 
jacent to the side seals, Fig. (5.1(a) , serves as a blocker to avoid air infusion into the land region in the middle of the bearing. Due to their large depth, the traditional theory assumed that the groove(s) act to isolate the land regions, i.e., the contribution of the grooves to the dynamics of SFDs were considered negligible and were treated as a constant ambient or supply pressure boundary condition for the land region(s) as exhibited in Fig. (5.2) [88, 1]. However, experimental studies have found that large dynamic pressures exist in the groove regions [89, 78, 76]. In reality, the flow pattern in a groove is very complicated and entails vortices traveling in the circumferential direction as depicted in Fig. (5.3). Attempting to capture this complex behavior in detail is very complicated and impractical for industrial purposes, and fortunately, it is also unnecessary. Instead, an approximation using the simple tool that Reynolds equation provides is sufficient. San Andres and Delgado [89] observed a virtual separation boundary delimited between the "circulation" area in the groove and an "axial flow" or "squeeze" body underneath. In fact, the incompressible circulation trapped in the groove acts as a filler material which acts to reduce the effective clearance of the lubricant that engages in the dynamic behavior. To compute the measured dynamic pressure in the groove, San Andres and Delgado solved the Reynolds equation with an "effective" groove depth, $d_{\text {eff }}$, which is much smaller than the physical depth exhibited in Fig. $5.3(\mathrm{~b})$. This approach is also adopted in this study by introducing a groove ratio parameter

$$
G_{r}=\frac{d_{e f f}}{c}
$$

where $c$ is the land clearance. 


\subsubsection{Orbit}

As previously mentioned in Sec. (1.2), journal rotation is suppressed in SFDs by an anti-rotation pin, as shown in Fig. (1.2), which makes SFDs incapable of providing hydrodynamic stiffness in the manner journal bearings do. More specifically, in the absence of external excitation, the journal in a SFD tends to sit at the bottom of the bearing. In order to avoid metal to metal contact, SFDs are often centered by means of a centering/retaining spring, which provides stiffness and a centering force which opposes the shaft weight. With the lack of journal rotation in the housing, some type of translatory whirl motion is the primary source which generates pressure. It is a common practice, especially for centered SFDs to assume the whirling motion to be circular and centered for analysis purposes. This has been shown to be a justifiable assumption based on real-time transient studies which have demonstrated steadystate convergence to a circular orbit [96, 97]. This journal trajectory is often called "CCO" for brevity. In this study, the journal center path is assumed to be CCO.

\subsection{Methodology and Governing Equations}

Based on Eq. (2.19), in the absence of journal rotation, the surface speed and consequently the convective inertia term is negligible. This reduces the two dimensional extended Reynolds equation 2.60 to

$$
\frac{\partial}{\partial x}\left(\frac{h^{3}}{k_{x} \mu} \frac{\partial p}{\partial x}\right)+\frac{\partial}{\partial z}\left(\frac{h^{3}}{k_{z} \mu} \frac{\partial p}{\partial z}\right)=\frac{\partial h}{\partial t}+I_{t}
$$

which predicts the squeeze and the temporal inertia effects as the only hydrodynamic pressure generating mechanisms in SFDs. In SFDs, flow is laminar, unless a large volume of air is ingested in the oil causing a considerable viscosity drop and Re rise. 
Hence, for standard operating conditions

$$
k_{x}=k_{z}=12
$$

which when coupled with Eq. (4.17) results in a simplified $I_{t}$ expression compared to Eq. (2.62)

$$
\begin{aligned}
& I_{t}=\frac{\rho h^{2}}{12 \mu} \frac{\partial^{2} h}{\partial t^{2}}-\frac{\rho h}{6 \mu} \frac{\partial h}{\partial x} \frac{\partial q_{x}}{\partial t} \\
& \text { primary temporal inertia: } \frac{\rho h^{2}}{12 \mu} \frac{\partial^{2} h}{\partial t^{2}} \\
& \text { secondary temporal inertia: }-\frac{\rho h}{6 \mu} \frac{\partial h}{\partial x} \frac{\partial q_{x}}{\partial t}
\end{aligned}
$$

Substituting Eq. (5.4) into Eq. (5.2) results in

$$
\frac{\partial}{\partial x}\left(h^{3} \frac{\partial p}{\partial x}\right)+\frac{\partial}{\partial z}\left(h^{3} \frac{\partial p}{\partial z}\right)=12 \mu \frac{\partial h}{\partial t}+\rho h^{2} \frac{\partial^{2} h}{\partial t^{2}}-2 \rho h \frac{\partial h}{\partial x} \frac{\partial q_{x}}{\partial t}
$$

where the secondary temporal inertia term is the last term in the right hand side of the equation. As previously mentioned, in the absence of external excitation, the journal of SFDs either rest at the bottom of the housing when there is no centering spring, or stays at a stationary point imposed by a centering spring. In either case, due to the lack of rotational speed, following the perturbation approach results in no "Zero" order equation (see Sec. (4.1)) and consequently no hydrodynamic stiffness values. For a preassumed trajectory ${ }^{1}$ e.g., CCO in this study, Eq. (5.7) acts as the "Auxiliary" equation which is solved for damping, added mass, and stiffnes: $\mathrm{f}^{2}$ coefficients. The

\footnotetext{
${ }^{1}$ For an unknown trajectory size, by coupling the rotordynamic analysis to the SFD analysis, an iterative scheme can be adopted to find the size of the orbit (but not the shape). However, a precise trajectory identification requires a full transient analysis

${ }^{2}$ This stiffness value is often an order of magnitude smaller than that of the centering spring is negligible.
} 
surface squeeze velocity and acceleration are calculated using Eqs. (2.5 \& 2.6). To calculate the secondary temporal inertia term though, Eq. 4.18 cannot be used, since there is no $p_{o}$ to base the formulation on. Thus, assuming

$$
Q_{x}=0.5
$$

in both uncavitated and cavitated regions is the simplest approach, and is therefore used in this study.

Traditionally two cavitation models are used in SFDs, $\pi$ and $2 \pi$ models. In the $\pi$ model, basically the negative portion of pressure profile is suppressed, while in $2 \pi$ model, the whole pressure profile is maintained. By the chose of the $2 \pi$ uncavitated bearing model, zero stiffness values are calculated. Based on the operating and sealing conditions of SFD, either model might be used. For example, it seems that for a SFD

with supply and discharge grooves on the sides, shown in Fig. $5.1(\mathrm{a}), 2 \pi$ model is more relevant. Recall that only having axial seals does not prevent air ingestion in most of the configurations, which deems $\pi$ model more relevant in most of the operating conditions. In addition, $\pi$ model by predicting damping values half of the $2 \pi$ model, is a more conservative model and is adopted in this study.

\subsubsection{Supply/Discharge Hole Models}

Three alternative models can be used to account for holes in SFDs. The simplest model is to assume that the holes enforce a constant static pressure, either a supply or discharge pressure depending on the hole type. As such, the finite element grid node(s) that correspond(s) to the hole location can be set to the hole pressure value as a boundary condition

$$
p_{h}=p_{s} \quad \text { or } \quad p_{h}=p_{d}
$$


This model is a fairly crude approximation of reality, since holes and nozzles are actually a source of pressure drop (e.g., in the case of feeding holes, the pressure that is provided to the clearance region is less than the supply pressure). Moreover, the lubricant pressure in the hole region in general is dynamic as well as the other regions and fixing it to a constant value can introduce significant errors in the results.

The other two methods of modeling holes both involve incorporating the hole flow into the Reynolds equation. This can be accomplished by adding an additional term to Eq. 5.7 as

$$
\frac{\partial}{\partial x}\left(h^{3} \frac{\partial p}{\partial x}\right)+\frac{\partial}{\partial z}\left(h^{3} \frac{\partial p}{\partial z}\right)=12 \mu \frac{\partial h}{\partial t}+\rho h^{2} \frac{\partial^{2} h}{\partial t^{2}}-2 \rho h \frac{\partial h}{\partial x} \frac{\partial q_{x}}{\partial t}-12 \mu u_{h}
$$

which applies to all elements which fall into the hole location. In Eq. (5.10), $u_{h}$ stands for the hole's average flow velocity, which is positive if the lubricant is fed into the SFD and negative for a discharge hole. Two different models can be used to determine $u_{h}$. Marmol and Vance [81] proposed a model that ties the flow velocity linearly to the pressure difference of the hole element and the supply/discharge pressure in the form

$$
u_{h}=C_{h}\left(p_{i}-p_{h}\right), \quad i=s, d
$$

where $C_{h}$ is the hole coefficient. In this model, the burden remains on the user to determine $C_{h}$. The other option is to use a nonlinear scheme based on the pressure drop equation in holes and nozzles. In this approach, $u_{h}$ can be expressed as

$$
u_{h}= \pm C_{h} \sqrt{\frac{2\left|p_{i}-p_{h}\right|}{\rho}}, \quad i=s, d
$$

where $C_{h}$ is also similarly the hole coefficient. He and Allaire [98] reported a CFD 
study that tied $C_{h}$ to the flow characteristics as

$$
\begin{aligned}
& C_{h}=\alpha \operatorname{Re}_{\text {hole }}{ }^{\beta}, \quad \operatorname{Re}_{\text {hole }}=\frac{\rho u_{h} D_{h}}{\mu} \\
& \beta=0.0014\left(\frac{L_{h}}{D_{h}}\right)+0.0031 \\
& \alpha= \begin{cases}0.1420\left(\frac{L_{h}}{D_{h}}\right)^{2}+0.0187\left(\frac{L_{h}}{D_{h}}\right)+0.581, & \frac{L_{h}}{D_{h}} \leq 1 \\
0.0013\left(\frac{L_{h}}{D_{h}}\right)^{2}-0.0369\left(\frac{L_{h}}{D_{h}}\right)+0.7773, & \frac{L_{h}}{D_{h}}>1\end{cases}
\end{aligned}
$$

In this work, the coefficient $\alpha$ was slightly tweaked from the original value found in [98] in order to achieve better consistency. The implementation of the nonlinear model requires an iterative scheme, hence, it is prone to potential convergence issues, which need to be avoided. However, the numerical evaluations of Eqs. (5.13 5.14 5.15 reveal that the $C_{h}$ varies between 0.5 and 0.8 and variation between these two limits have meager effects on the hole model. Therefore, it is recommended to estimate $C_{h}$ value to simplify the calculations and improve the solution convergence.

The effects of the holes, however, diminish as the depth of the groove(s) that they open into increases. As previously mentioned, the dynamics of grooves are very complicated and the holes' contribution as a part of this complex system is very difficult to assess, but can usually be ignored for deep grooves. For many SFDs, the holes are located in deep grooves, and are isolated by the "circulation" area from the the thin "squeeze" body that generates the dynamic pressure. Therefore, one can model the deep grooves and ignore the hole effects for these cases. 


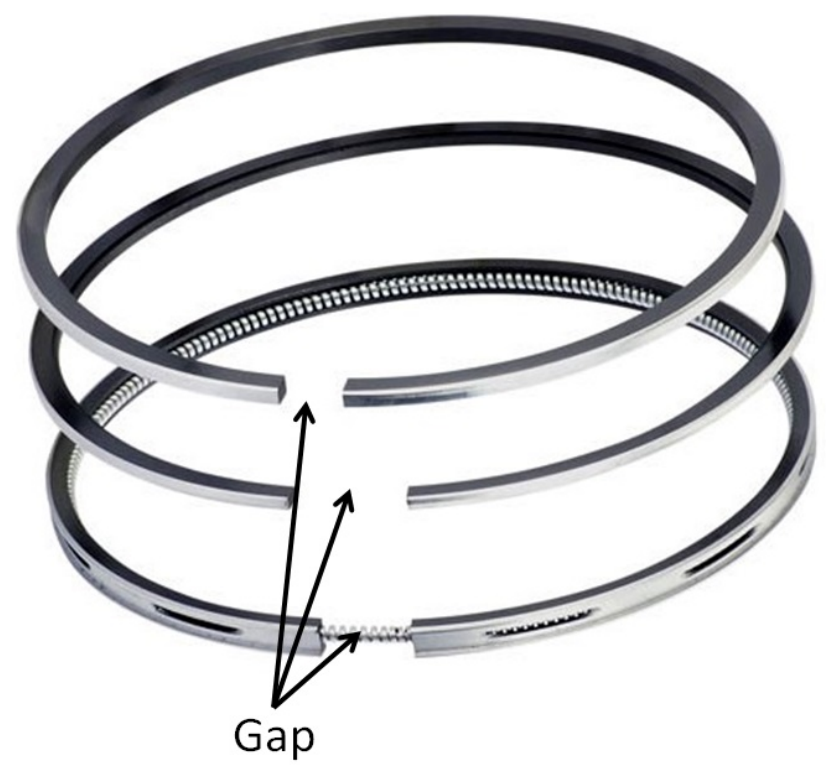

Figure 5.4: Piston ring

\subsubsection{Axial End Models}

In SFDs with open ends, pressure drops to ambient at the axial ends

$$
p_{@ z= \pm \frac{L}{2}}=p_{a}
$$

which serves as a constant pressure boundary condition for the FE model. As noted previously, in order to reduce side leakage, axial seals can be mounted in the SFD geometry. The most common type of axial seal used in SFDs is the piston ring (Fig. (5.4)). A piston ring is an incomplete ring that sits in a groove and acts to reduce the leakage by tightening the clearance. The leakage through a piston ring depends upon the pressure difference at the two sides of the ring and its physical properties. At the gap where the seal is left open, the lubricant is directly exposed to the ambient pressure. For the nodes that correspond to this gap, Eq. (5.16) can be used as a constant pressure boundary condition. Marmol and Vance [81] proposed a 


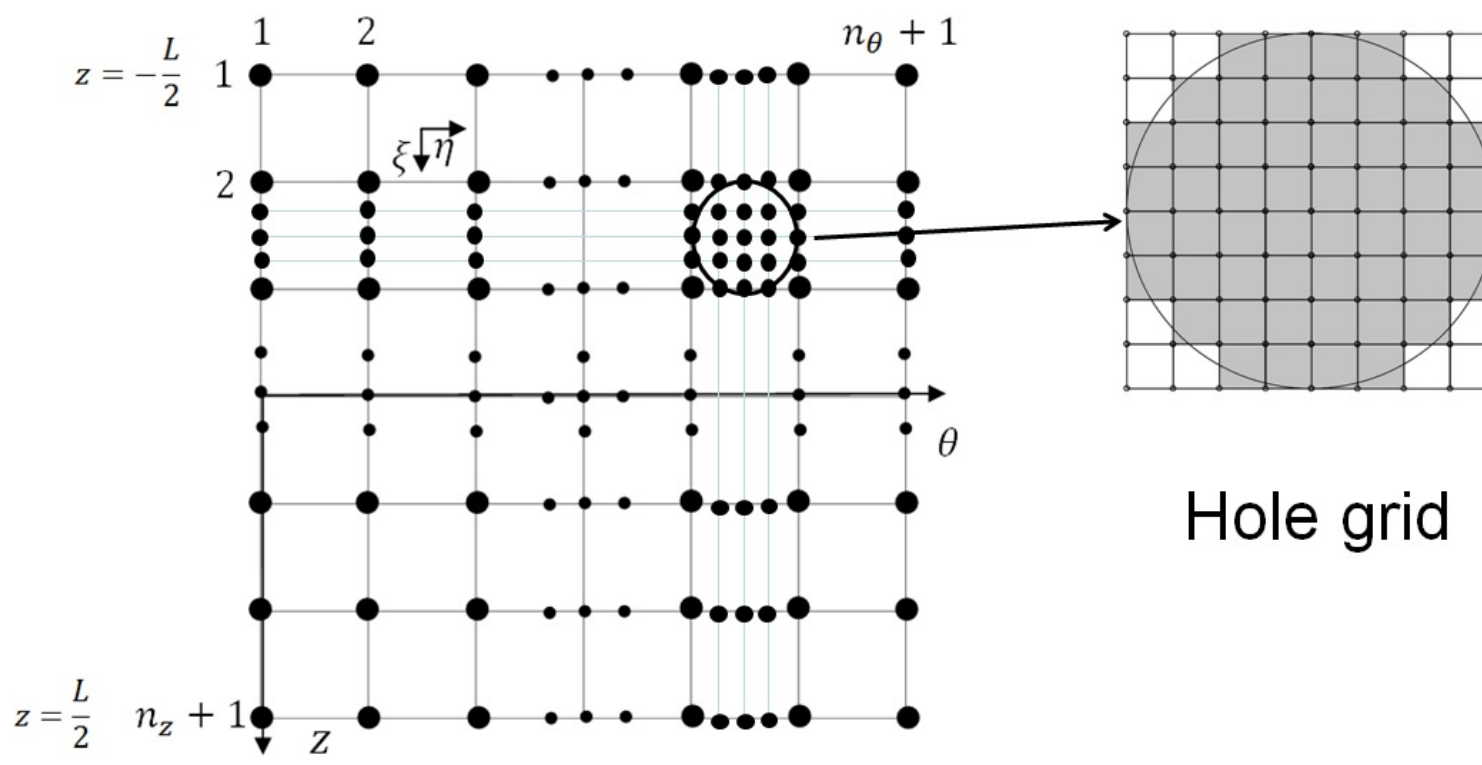

Figure 5.5: Mesh grid in MAXSFD

simple formula to quantify the piston ring leakage

$$
\frac{d p}{d z}=C_{p} \frac{p_{a}-p}{w_{p}}\left(\frac{h_{p}}{h}\right)^{3}
$$

This equation provides a derivative or Neumann boundary condition at the nodes corresponding to the seal location (i.e., $@ z= \pm \frac{L}{2}$ ), other than the open gap area. $C_{p}$ is the piston coefficient that depends upon its properties and $w_{p}$ is the ring width in the axial direction. The film thickness around the piston ring is defined in a similar manner to Eq. (2.4)

$$
h_{p}=c_{p}-X \cos (\theta)-Y \sin (\theta)
$$

which describes the circumferentially varying film thickness as a function of the journal displacement $(X, Y)$. 


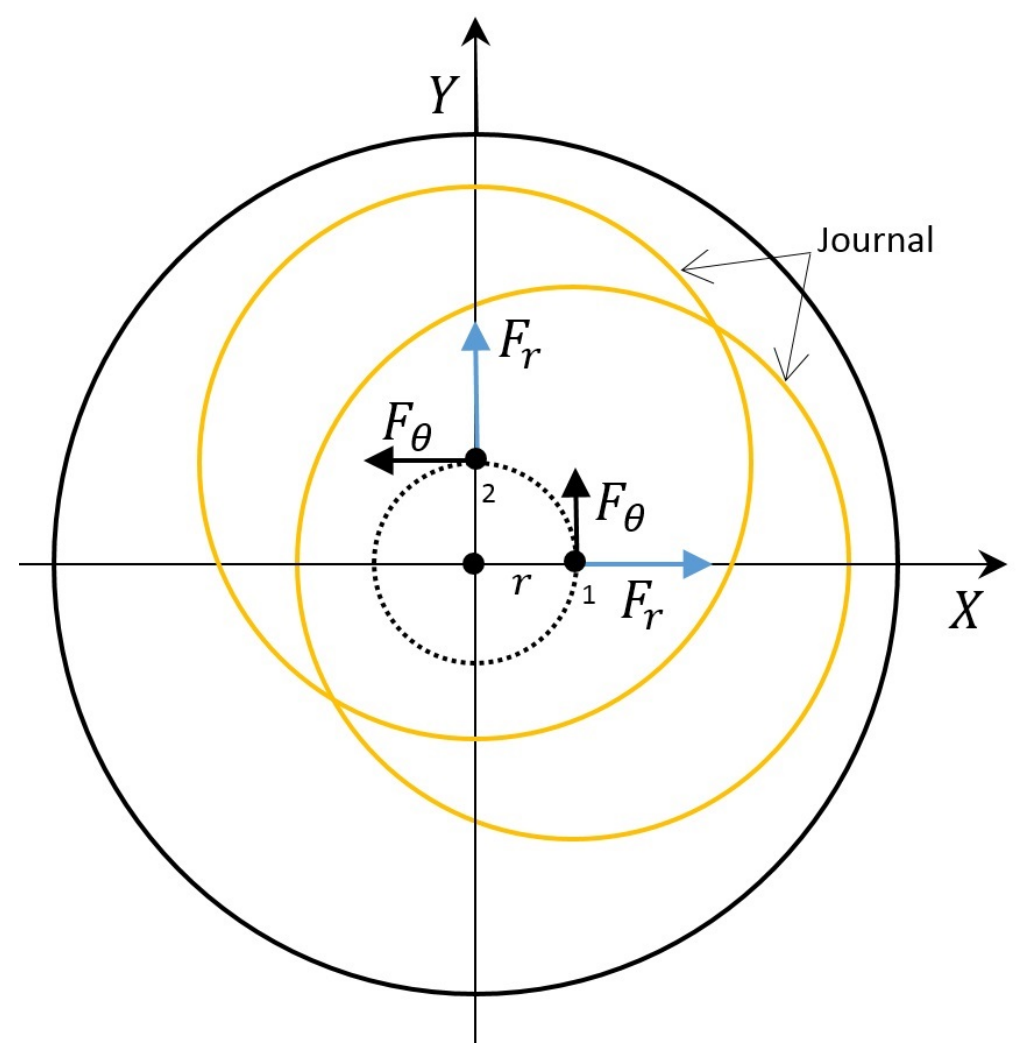

Figure 5.6: Circular centered orbit

\subsection{MAXSFD}

To implement the theory that was outlined in the previous section, a computer code named MAXSFD was developed. This program is a finite element based code that solves the Reynolds equation (5.7) in two dimensions (circumferential and axial). This code includes a smart meshing feature that refines the mesh in the hole and piston ring gap area to a user defined resolution. This feature enables the avoidance of sharp unrealistic peaks at the location of holes as depicted in the pressure profile plots by He [83]. By specifying the hole location and the minimum number of elements to be used, MAXSFD automatically determines which nodes fall in a hole region and redefines the mesh grid in this region (see Fig. (5.5)), as well as associating the user-defined modified equation (either 5.10 or $(5.9)$ ) to the relevant nodes. 
MAXSFD determines the pressure profile by assuming CCO as the journal whirling trajectory. $\mathrm{CCO}$ is axisymmetric, but the arrangement of holes and piston ring seals may break this symmetry. However, their effects are found in general to be small enough to allow the radial and tangential forces calculated at different angles of the CCO to be effectively invariant. Analysis is performed based on the radial and tangential forces of the lubricant exerted on the journal as depicted in Fig. (5.6). Although these forces are in general nonlinear with displacement, i.e., the radius of $\mathrm{CCO}$, for linear rotordynamic analysis purposes, it is a common practice to represent these forces (especially for small amplitude motions) with a set of linear coefficients [10, 76, 78, 80, 95]. Two approaches for determination of these linear coefficients can be adopted: i) whirl speed dependent approach and ii) whirl speed independent approach.

\subsubsection{Whirl Speed Dependent Approach}

In the first method, which is an extended version of the method by Barrett and Gunter [1] used in the ROMAC code SQFDAMP, the cross-coupled coefficients are ignored and the radial and tangential forces are linearized by using added mass $M$, damping $C$, and stiffness $K$ terms as follows

$$
\begin{aligned}
& \frac{F_{r}}{r}=K-\Omega^{2} M \\
& \frac{F_{\theta}}{r}=C \Omega
\end{aligned}
$$

Calculating damping coefficient $C$ is straightforward by the use of Eq. (5.20). In order to calculate $K$ and $M$, Reynolds equation needs to be solved twice, once without the temporal inertia terms included in Eq. (5.7), so that no added mass term is present. 
The resulting radial force, $F_{r 1}$, represents the (dynamic) stiffness only

$$
K=\frac{F_{r 1}}{r}
$$

With $K$ determined, a second Reynolds equation solution can be conducted with all of the terms of Eq. (5.7) included. Based on this second resulting radial force $\left(F_{r 2}\right)$, $M$ maybe determined by the following equation

$$
M=\frac{K}{\Omega^{2}}-\frac{F_{r 2}}{r \Omega^{2}}
$$

Two points are worth mentioning here. First, as was stated previously, SFDs do not possess stiffness properties in the manner of common radial bearings. The dynamic stiffness in Eq. (5.21) is the radial restoration force manifestation of the SFD whirling in an assumed "CCO" in the presence of a centering spring. The magnitude of this coefficient is much smaller compared to the stiffness of the centering spring, $K_{s}$. Second, the proposed identification approach generates a set of coefficient per whirling speed, $\Omega$. This is the chosen approach implemented in MAXSFD.

\subsubsection{Whirl Speed Independent Approach}

This method relies on the KCM coefficient identification method derived in detail in Appendix. C. Based on Eqs. (C.7 C.8 C.10 \& C.11), the SFD's force components with both principle and cross-coupled linear coefficients included can be express as

$$
\begin{aligned}
& -M \Omega^{2}+c \Omega+K=\frac{F_{r}}{r} \\
& m \Omega^{2}+C \Omega-k=\frac{F_{\theta}}{r}
\end{aligned}
$$




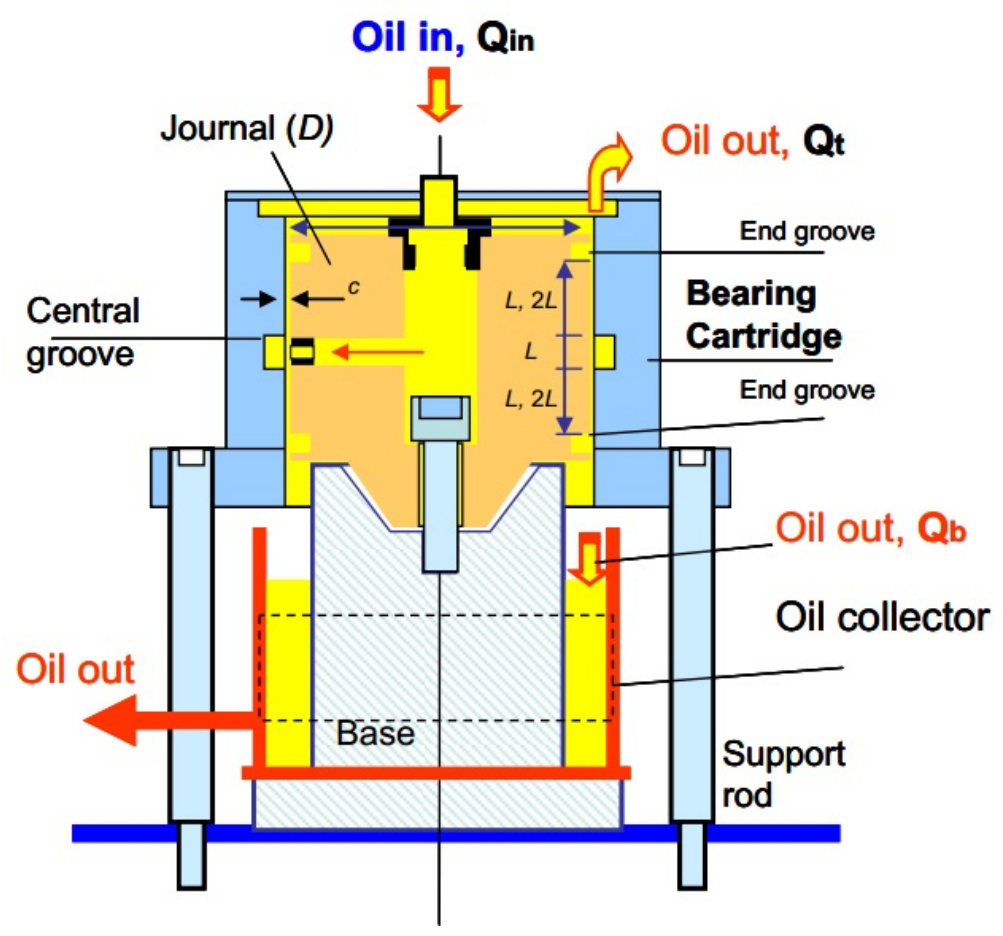

Figure 5.7: Texas A\&M, SFD test rig configuration [78]

In order to solve for the coefficients $(M, m, C, c, K, k), \frac{F_{r}}{r}$ and $\frac{F_{\theta}}{r}$ are approximated by a quadratic curve-fit over a span of whirling frequencies. This method is based upon the assumption that the coefficients remain invariant over a range of whirling speeds. The drawback of this method is that in general, this assumption may not hold, especially for large whirl amplitudes $(r)$ and wide ranges of while speeds $(\Omega)$.

\subsection{Results}

A series of tests have been conducted in the Turbomachinery Laboratory at Texas A\&M University, which investigate various SFD configurations. The associated test rig, depicted in Fig. (5.7), was designed to use a series of swappable fixture rods which provide centering stiffnesses in the range of $15(\mathrm{MN} / \mathrm{m})<K_{s}<25(\mathrm{MN} / \mathrm{m})$. The rig has a bearing cartridge supported atop the centering elastic rods and a stationary 


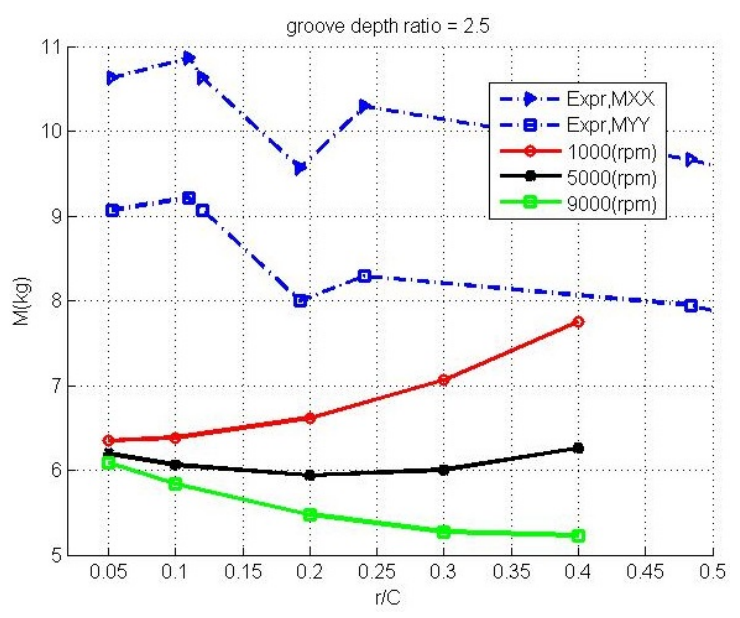

(a) $M$, short, open ends, $G_{r}=2.5$

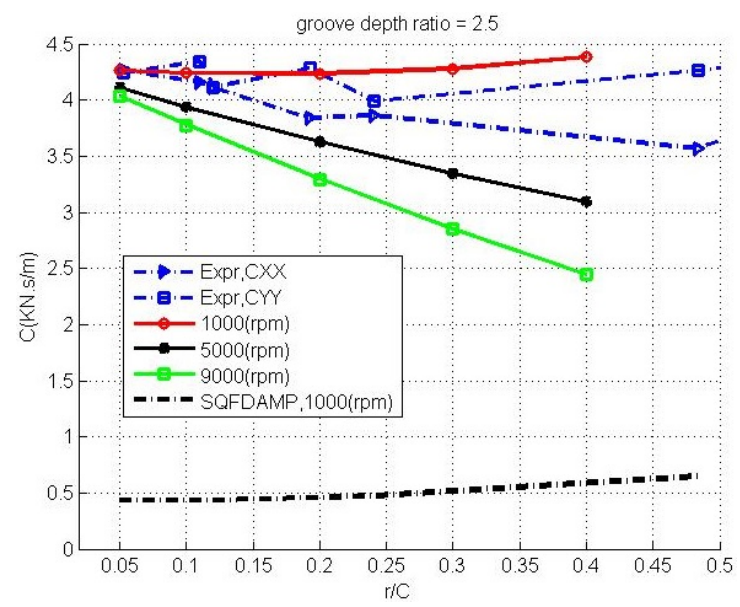

(c) $C$, short, open ends, $G_{r}=2.5$

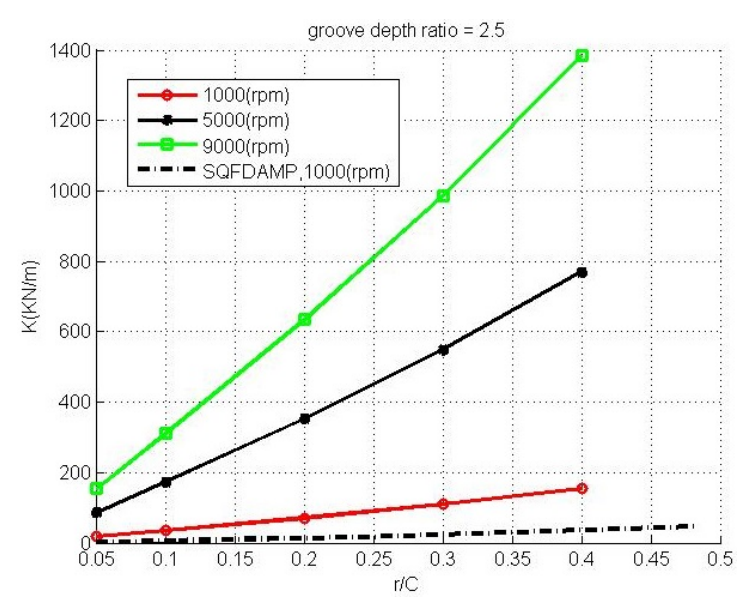

(e) $K$, short, open ends, $G_{r}=2.5$

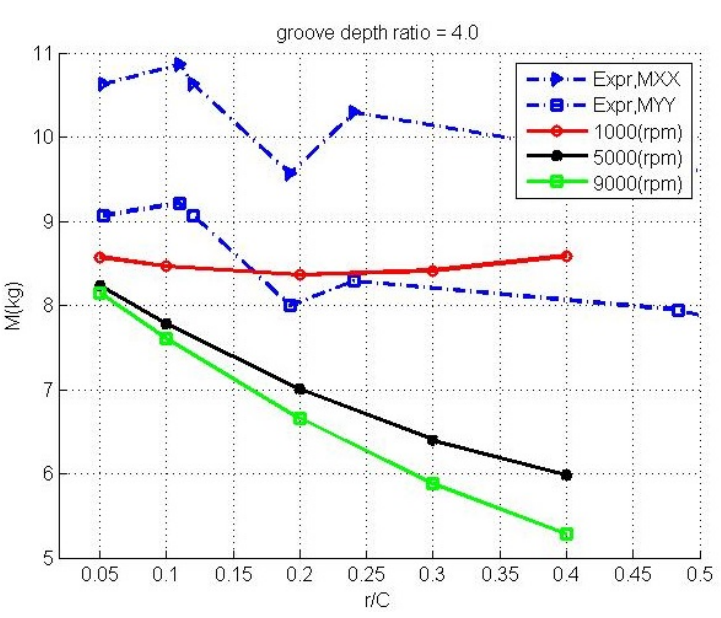

(b) $M$, short, open ends, $G_{r}=4.0$

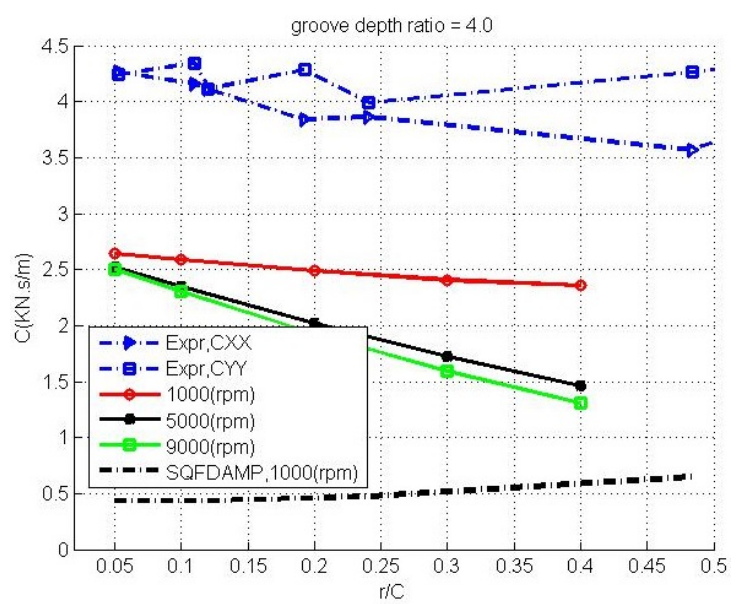

(d) $C$, short, open ends, $G_{r}=4.0$

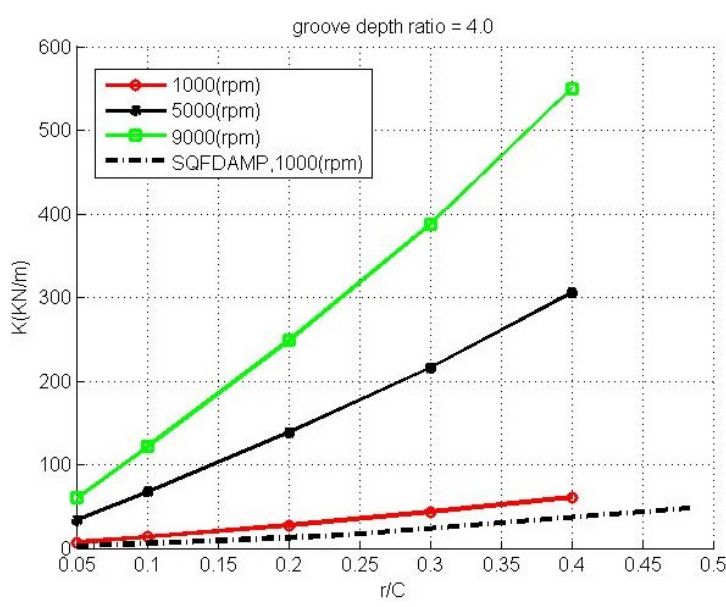

(f) $K$, short, open ends, $G_{r}=4.0$

Figure 5.8: Short open end SFD, linear coefficients vs. whirl amplitude ratio, results from MAXSFD, SQFDAMP and test data from [78. 


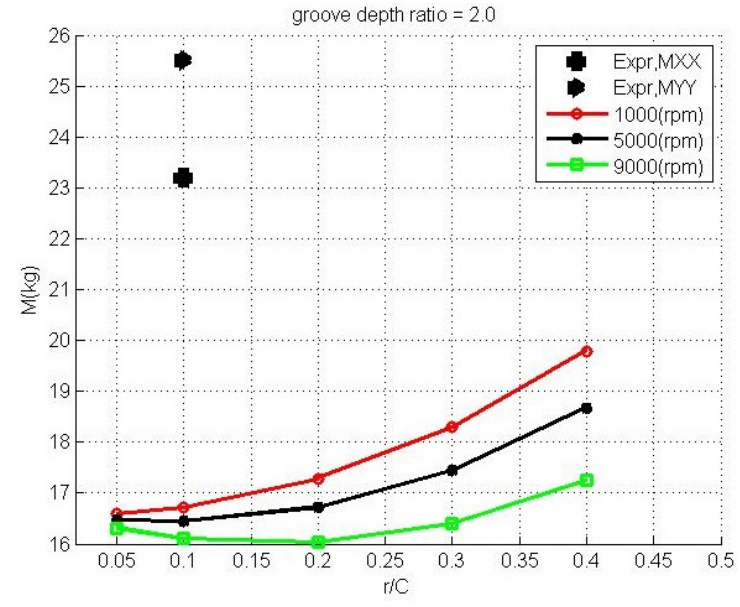

(a) $M$, long, open ends, $G_{r}=2$

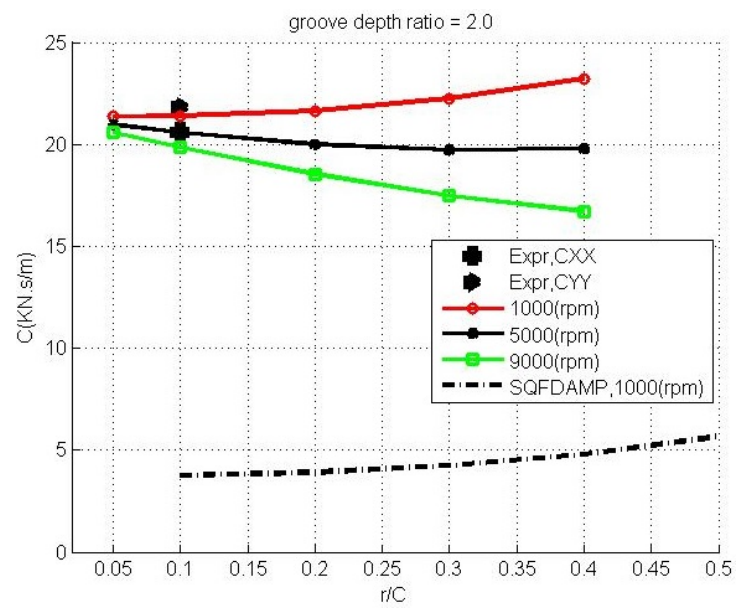

(c) $C$, long, open ends, $G_{r}=2$

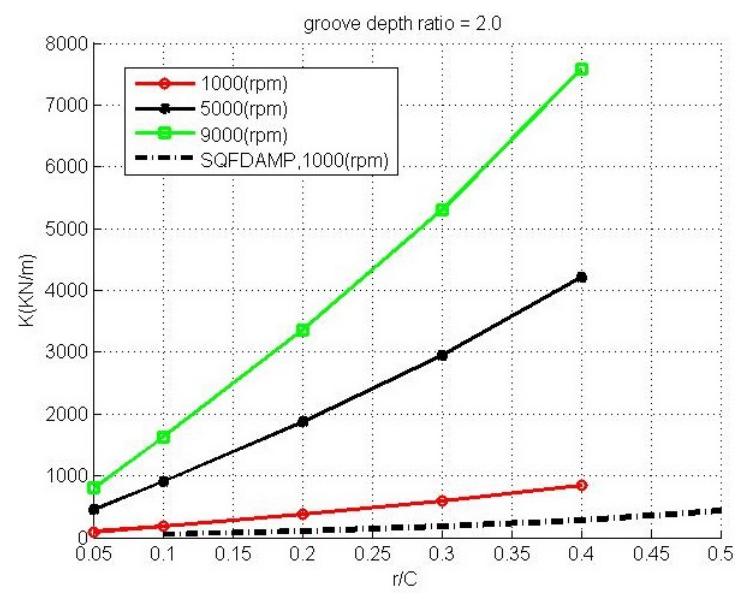

(e) $K$, long, open ends, $G_{r}=2$

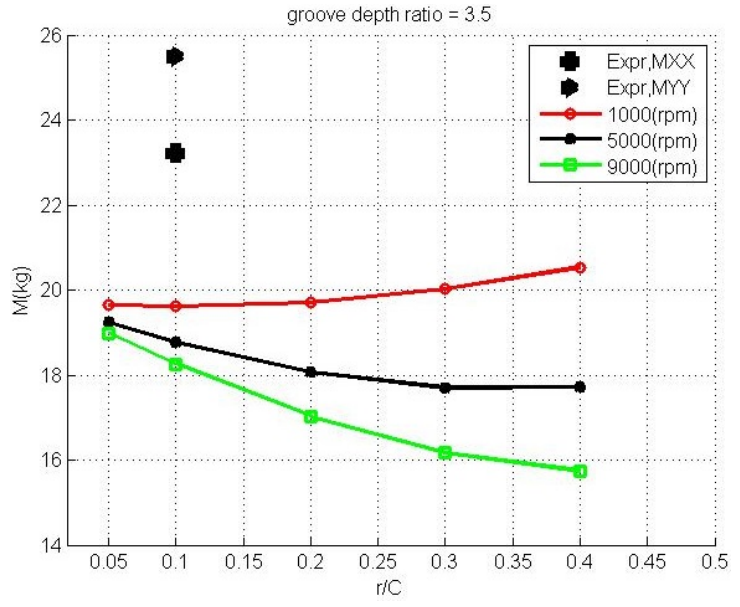

(b) $M$, long, open ends, $G_{r}=3.5$

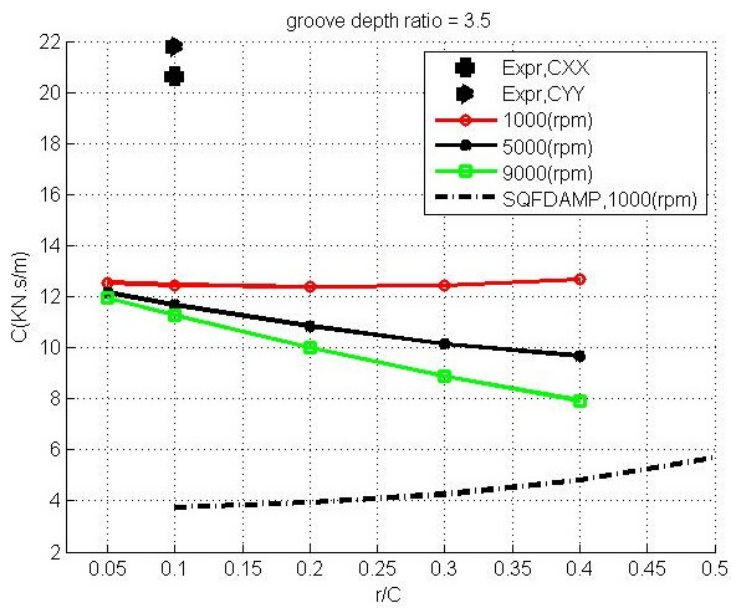

(d) $C$, long, open ends, $G_{r}=3.5$

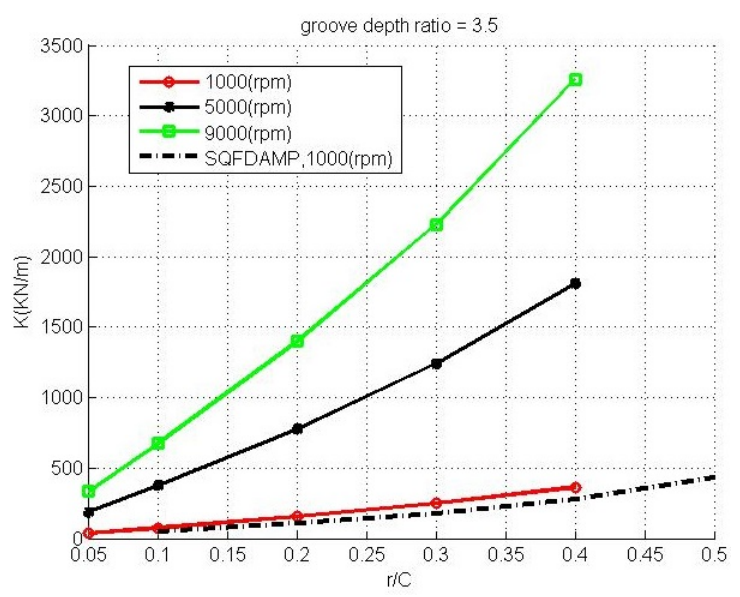

(f) $K$, long, open ends, $G_{r}=3.5$

Figure 5.9: Long open end SFD, linear coefficients vs. whirl amplitude ratio, results from MAXSFD, SQFDAMP and test data from [78] 


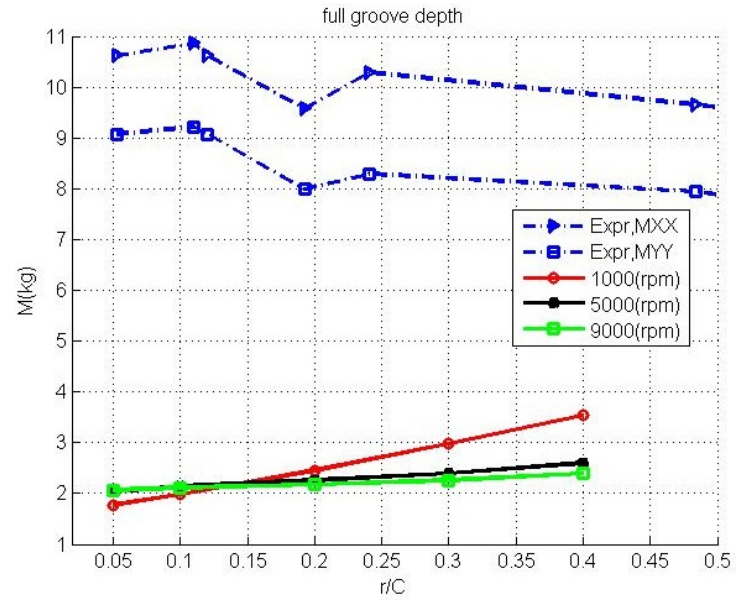

(a) Short, open, full groove

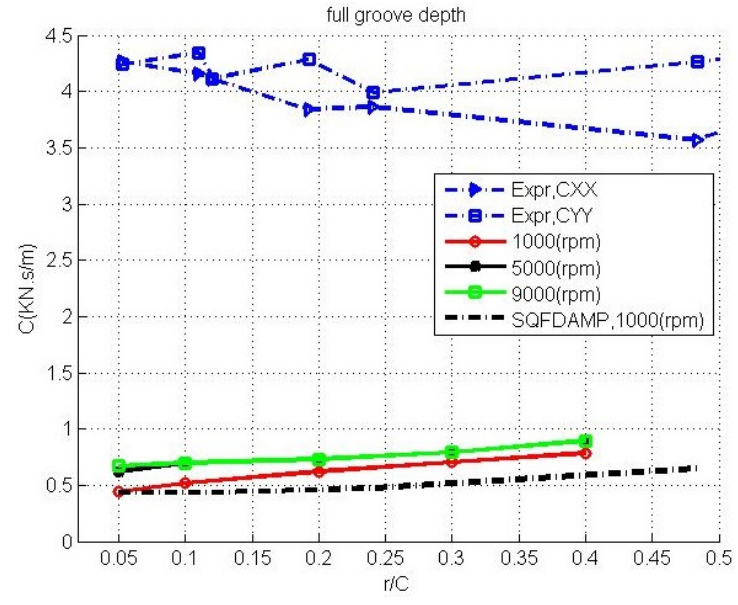

(c) Short, open, full groove

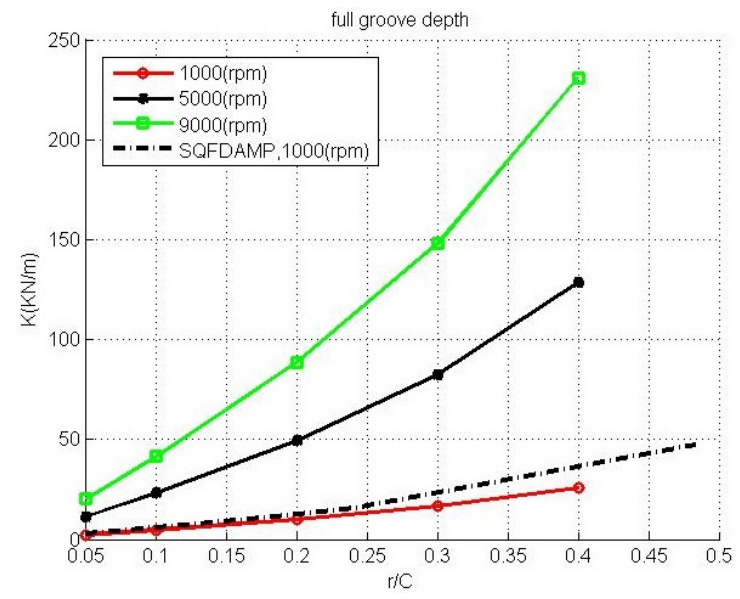

(e) Short, open, full groove

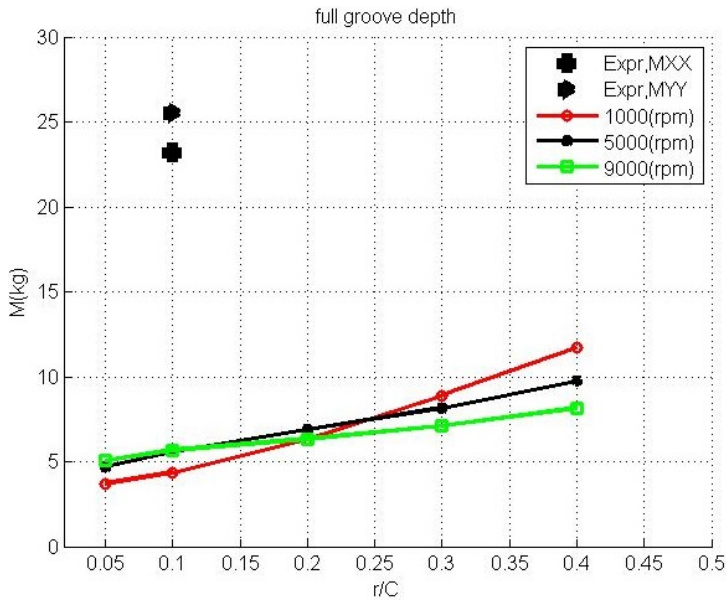

(b) Long, open, full groove

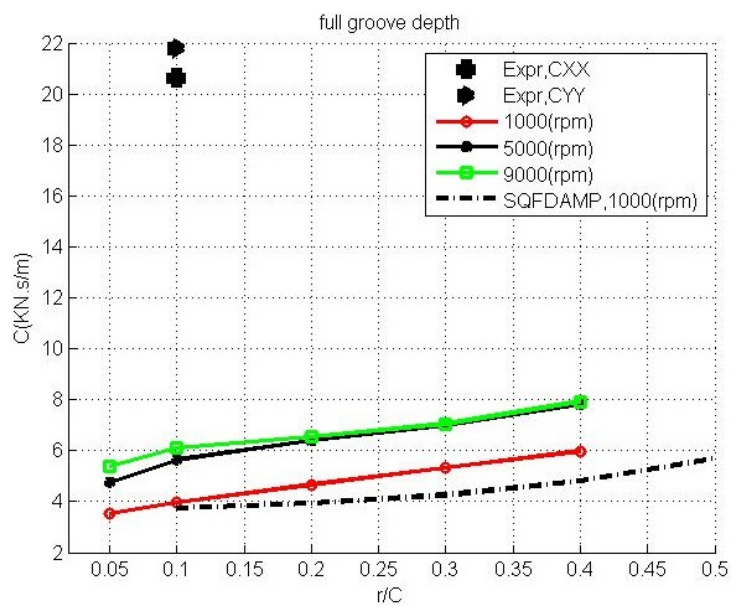

(d) Long, open, full groove

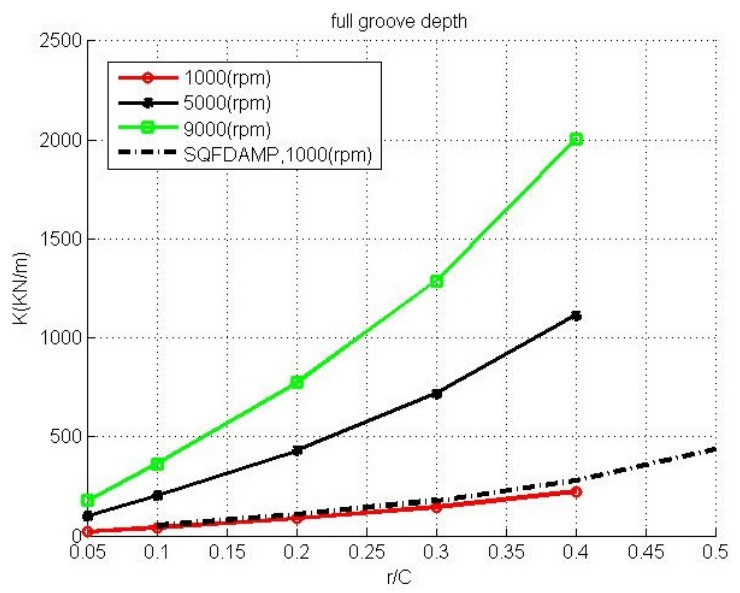

(f) Long, open, full groove

Figure 5.10: Short and long open end SFD, linear coefficients vs. whirl amplitude ratio with $d_{e f f}=9.5(\mathrm{~mm})$, results from MAXSFD, SQFDAMP and test data from [78] 
Table 5.1: Texas A\&M test rig characteristics [78, 76]

\begin{tabular}{c|c|c}
\hline \hline & short SFD & long SFD \\
\hline Journal Diameter & $127(\mathrm{~mm})$ & $127(\mathrm{~mm})$ \\
\hline Land Length & $12.7(\mathrm{~mm})$ & $25.4(\mathrm{~mm})$ \\
\hline Radial clearance & $0.141(\mathrm{~mm})$ & $0.138(\mathrm{~mm})$ \\
\hline Groove Axial Length & $12.7(\mathrm{~mm})$ & $12.7(\mathrm{~mm})$ \\
\hline Groove Physical Depth & $9.5(\mathrm{~mm})$ & $9.5(\mathrm{~mm})$ \\
\hline Lubricant Density & $785\left(\mathrm{Kg} / \mathrm{m}^{3}\right)$ & $785\left(\mathrm{Kg} / \mathrm{m}^{3}\right)$ \\
\hline Lubricant Viscosity & $2.96(\mathrm{mPa} . \mathrm{s})$ & $2.96(\mathrm{mPa} . \mathrm{s})$ \\
\hline Lubricant Supply Temperature & $25\left({ }^{\circ} \mathrm{C}\right)$ & $25\left({ }^{\circ} \mathrm{C}\right)$ \\
\hline Static Groove Pressure $($ end sealed $)$ & $0.52(\mathrm{bar})$ & $0.72(\mathrm{bar})$ \\
\hline Static Groove Pressure $($ open end $)$ & $0.76(\mathrm{bar})$ & $4.69(\mathrm{bar})$ \\
\hline Piston Ring Coefficient & 0.0525 & 0.0069
\end{tabular}

journal in the middle, rigidly attached to a base structure. The test article SFDs consist of two parallel film lands separated by a central groove which is $12.7 \mathrm{~mm}$ (0.5 in) wide and $9.5 \mathrm{~mm}(3 / 8 \mathrm{in})$ deep and is illustrated in Figs. (5.1(c) $\& 5.1(\mathrm{~d})$. Two different configurations have been tested:

i. a "short" 3 SFD with $12.7 \mathrm{~mm}(0.5 \mathrm{in})$ long lands,

ii. a "long" SFD with $25.4 \mathrm{~mm}$ (1.0 in) long lands.

In the journal, there are three equally spaced holes $\left(120^{\circ}\right.$ apart $)$, which supply a light oil (ISO VG 2) into the central groove and squeeze film lands [78]. The low viscosity oil simulates high temperature operation of the SFD. The test SFDs contain two axial end piston ring grooves that are left vacant resulting in open end configurations. More details on the instrumentation, test rig, parameter identification procedure, and test procedure can be found in [75, 77].

\footnotetext{
${ }^{3}$ The reader should distinguish between short and long bearing analysis assumptions, and the "short" and "long" terms used here to imply the length of the SFD configuration
} 
The purpose of this work is to compare, contrast and validate the results of MAXSFD against the experimental data. In addition, the data is used to verify the effects of the central feeding groove on the dynamics of SFDs. As previously mentioned, the traditional assumption is that the central feeding groove isolates the adjacent film lands and provides "no" dynamic pressure as depicted in Fig. (5.2). Recall that based on this assumption, the groove region may supply high static pressure to the SFD, however, this pressure does not contribute to the dynamics of the bearing except through the suppression of cavitation.

For each whirl amplitude $(r)$, the experimental dynamic coefficients by Texas A\&M turbomachinery group were extracted and assumed invariant over a range of whirl speed $(\Omega)$. This assumption is appropriate for small amplitudes, but for larger amplitudes, it may not hold. The dynamic coefficients for "CCO" reported in the references cover a range of whirl amplitudes $r$ for only the short open-end SFD. For all the other cases listed in Table (5.1), coefficients pertaining to just one single small $r$ are reported. The numerical results of MAXSFD are reported for "CCO" and the dynamic coefficents are extracted based on the whirl speed dependent method. The physical groove ratio of the tested SFDs is $G_{r} \sim 70$, which signifies a deep groove. The results of MAXSFD are obtained for a range of $1 \leq G_{r} \lesssim 70$ and the best match with experimental results is plotted. Since the groove is deep, the hole model is excluded from the analysis for the sake of simplicity, however, one may choose to apply the experimentally measured static pressure reported in Table (5.1) to the supply hole nodes based on Eq. (5.9).

Figure (5.8) depicts the linear coefficients of the short open end SFD for $G_{r}=$ 2.5, 4.0, and $\Omega=1000,5000,9000(\mathrm{rpm})$. It can be observed from Fig. (5.8(c) that $G_{r}=2.5$ yields the best match of the numerical and experimental damping coefficients; however, the numerical added mass values are $\sim 30 \%$ lower than the experi- 
mentally obtained values. On the other hand, by increasing the effective groove depth to $G_{r}=4.0$, better correlation is achieved for $M$ values, while $C$ is underestimated by about $\sim 50 \%$. In addition to MAXSFD results, a tradition SFD code (SQFDAMP) was also used for comparison against experimental data. SQFDAMP underpredicts the damping in both $G_{r}$ cases, but is incapable of assessing $M$ due to the adoption of the short bearing solution of the traditional Reynolds equation in its formulation. Recall that the stiffness values are a small fraction of the centering spring stiffness for small $r$ s and low $\Omega \mathrm{s}$, and are therefore of much less importance than the $C$ and $M$ values.

The only "CCO" experimental data available for long open end SFDs is related to the small value of $\frac{r}{c}=0.1$. Figure 5.9 illustrates the long SFD results, which are very similar to the previous short SFD results depicted in Figure (5.8). For the long open end SFD, $G_{r}=2$ successfully predicts the $C$ test values. The closest and best match with the mass test values is obtained when $G_{r}=3.5$. The mass values are underpredicted by $\sim 10 \%$. SQFDAMP underpredicts the $C$ values for both $G_{r}$ cases, but closely follows the $K$ trend of MAXSFD. Figure (5.10) depicts the results obtained based on the unrealistic assumption that $d_{\text {eff }}$ equals the groove full depth, that is, no circulation area exists and the entire groove depth actively participates in the SFD dynamics. The obtained results underpredict both $M$ and $C$, and additionally, a very good correlation of the $K$ and $C$ results between MAXSFD and SQFDAMP are achieved when the unrealistic the full groove depth is utilized as $d_{\text {eff }}$. From this result it can be concluded that assuming the entire body of the lubricant in the groove participates in the dynamics of the SFD, indeed does isolate the lands.

Figures 5.11 5.13) depict results associated with the sealed short and long SFDs. In Fig. (5.11), related to the short sealed SFD, $G_{r}=3.5$ reveals a close match with 


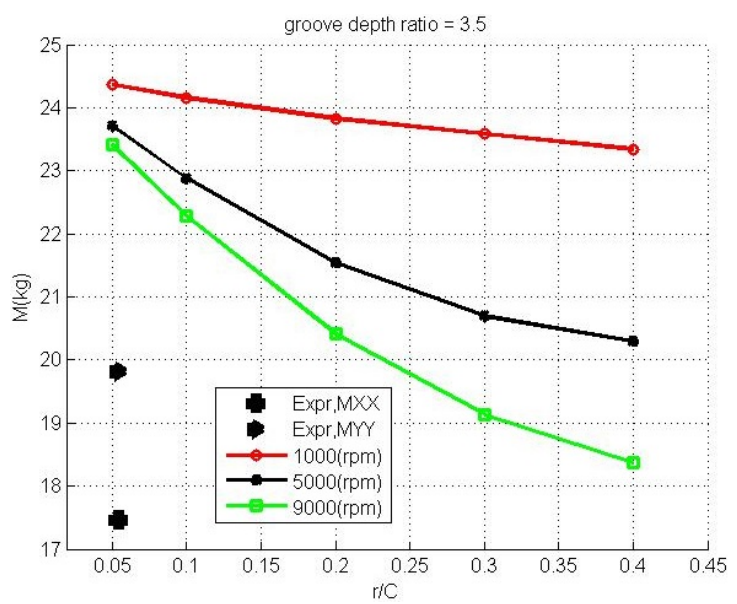

(a) Short, sealed ends, $G_{r}=3.5$

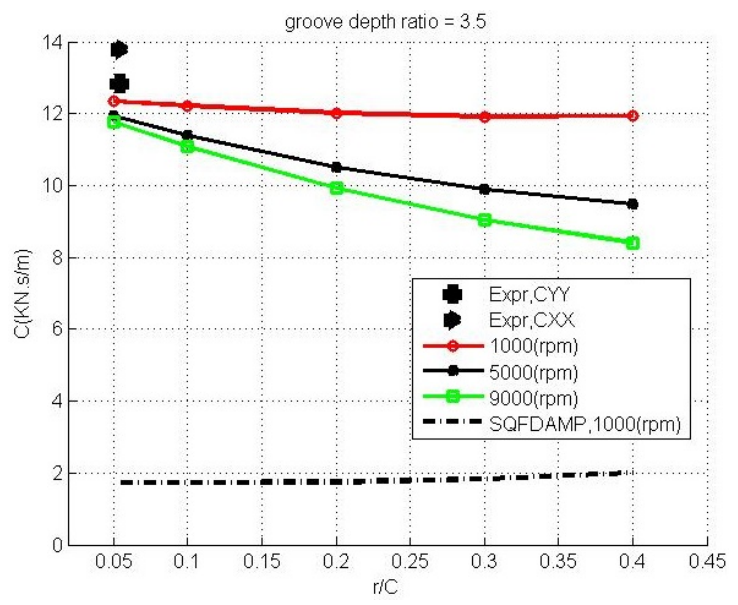

(c) Short, sealed ends, $G_{r}=3.5$

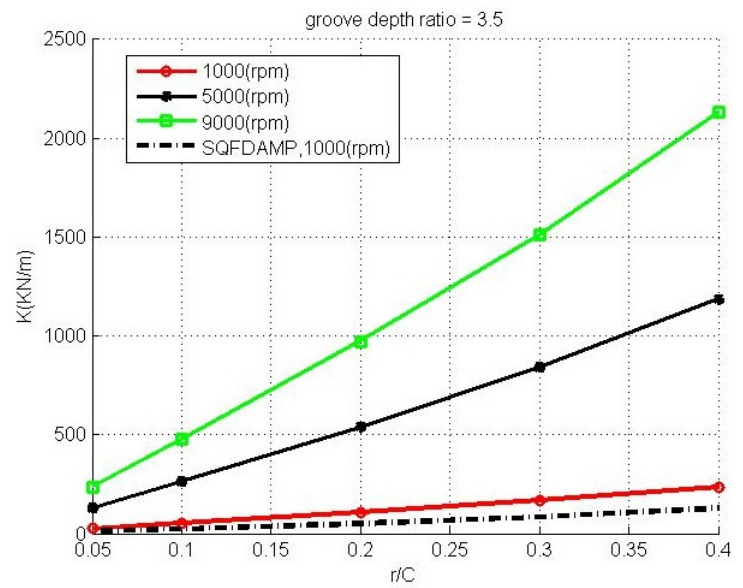

(e) Short, sealed ends, $G_{r}=3.5$

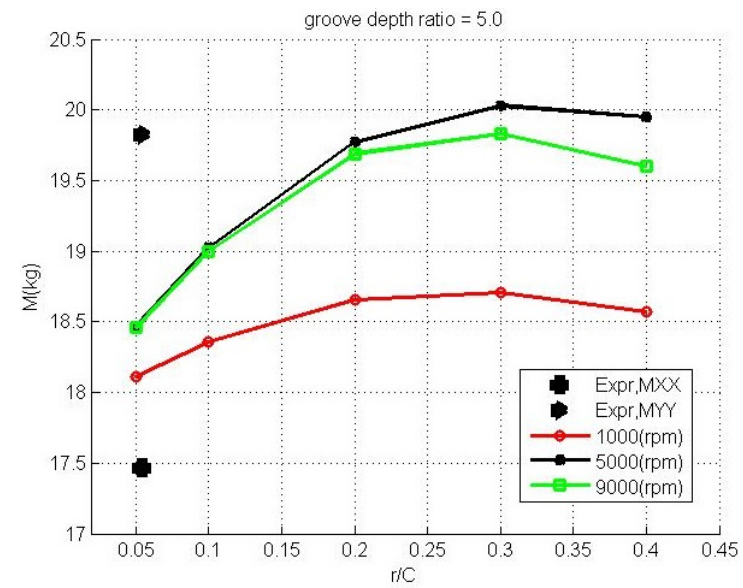

(b) Short, sealed ends, $G_{r}=5$

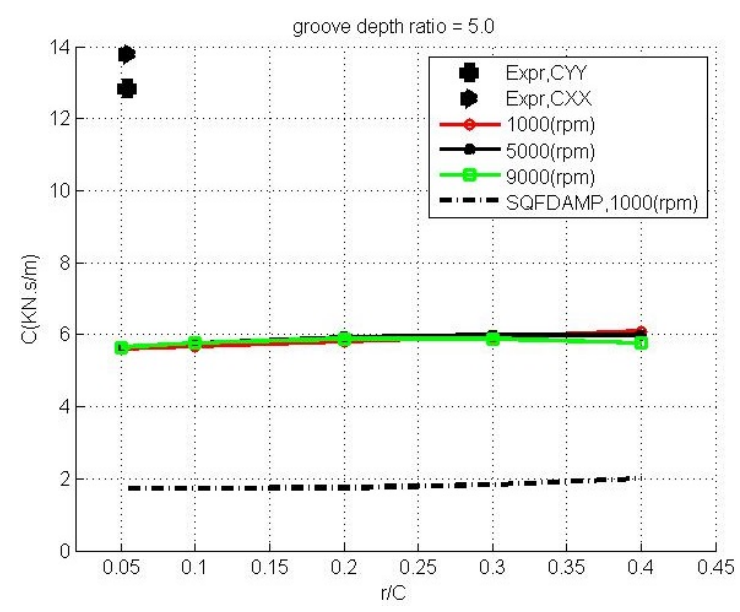

(d) Short, sealed ends, $G_{r}=5$

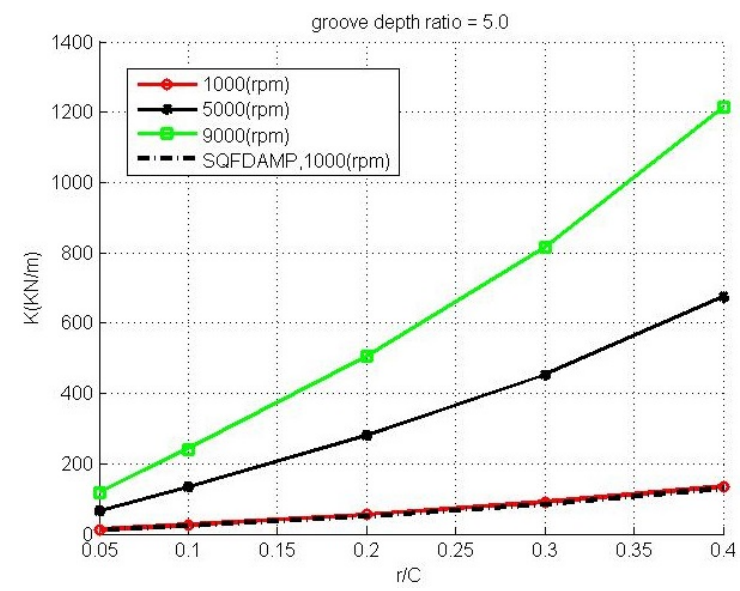

(f) Short, sealed ends, $G_{r}=5$

Figure 5.11: Short sealed end SFD, linear coefficients vs. whirl amplitude ratio, results from MAXSFD, SQFDAMP and test data from [76] 


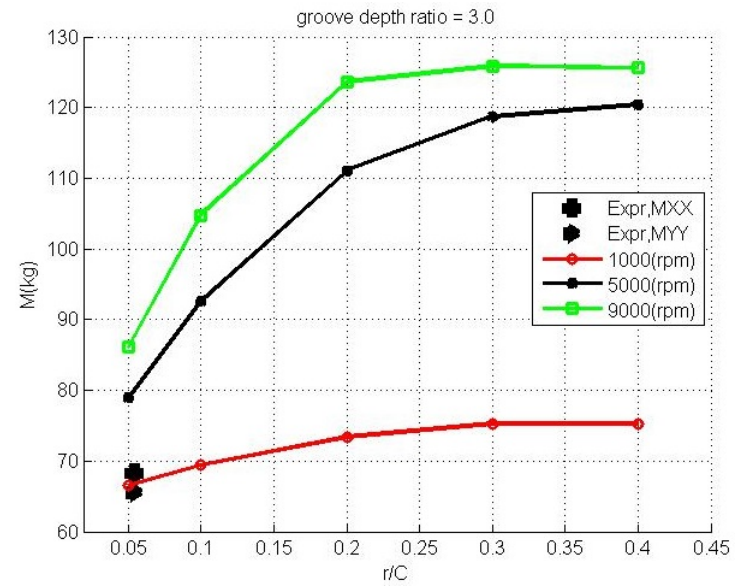

(a) Long, sealed ends, $G_{r}=3$

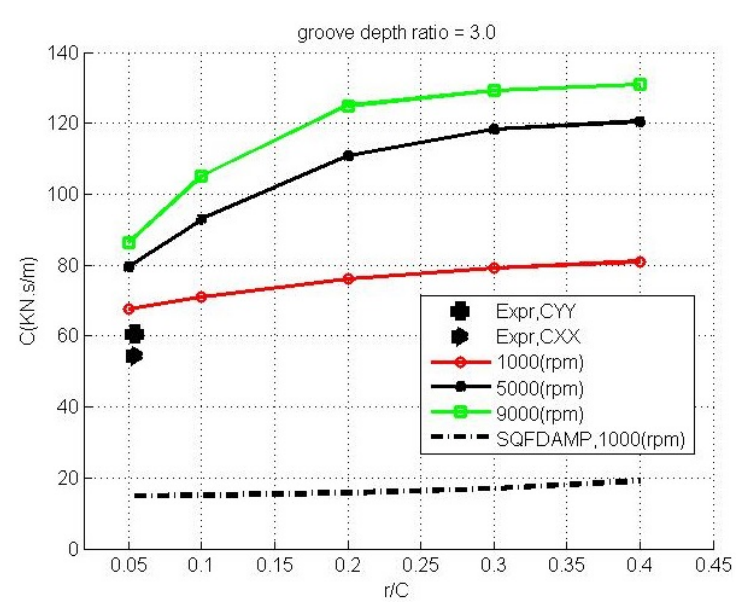

(c) Long, sealed ends, $G_{r}=3$

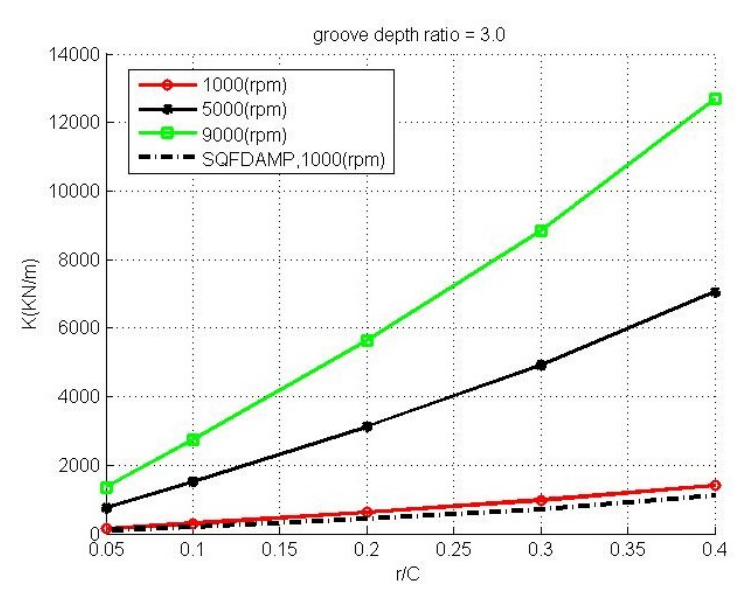

(e) Long, sealed ends, $G_{r}=3$

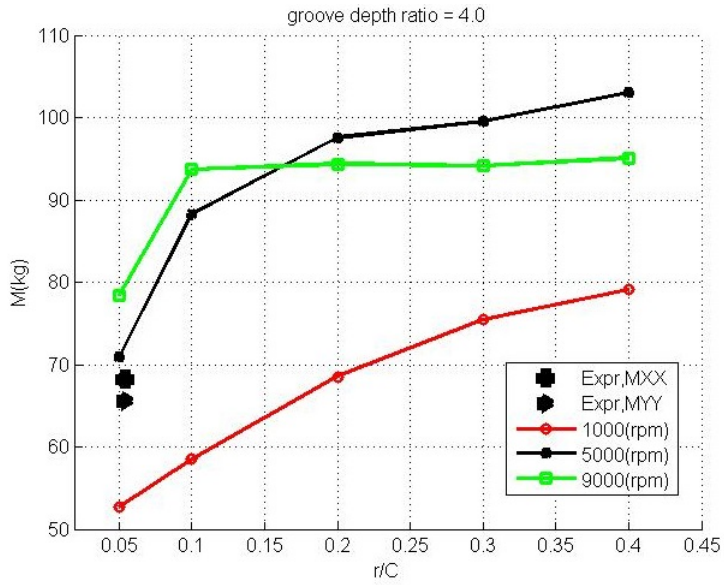

(b) Long, sealed ends, $G_{r}=4$

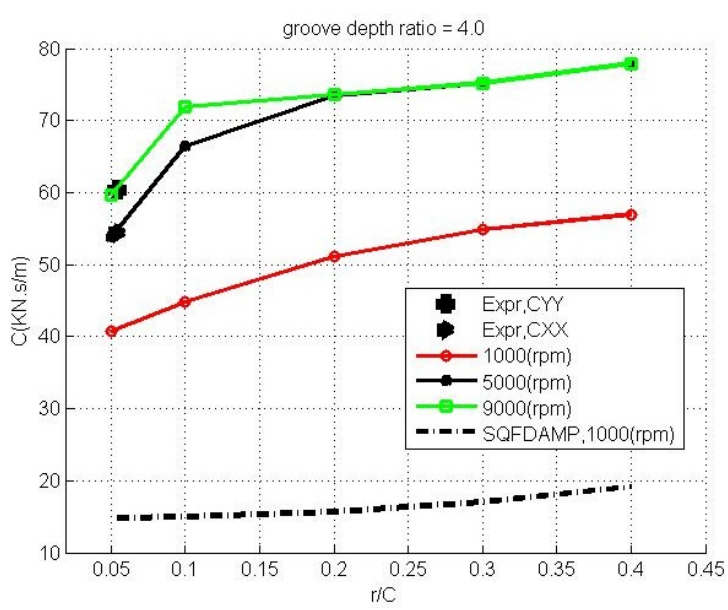

(d) Long, sealed ends, $G_{r}=4$

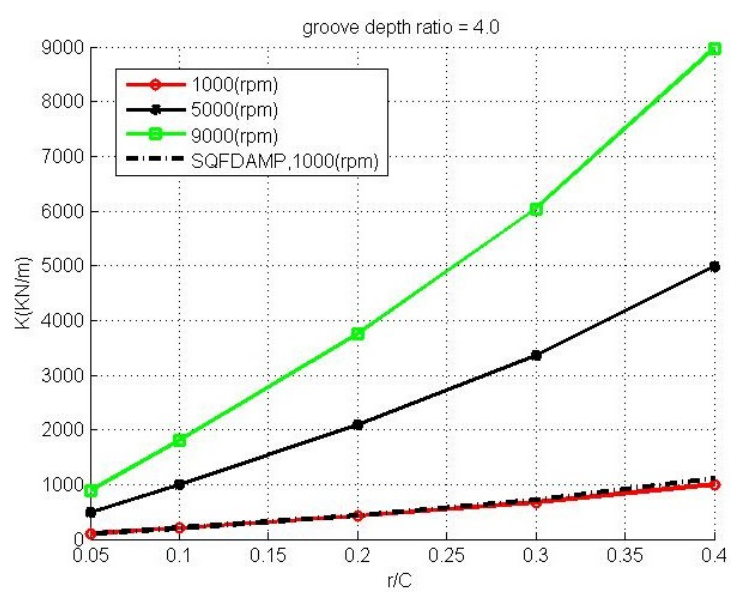

(f) Long, sealed ends, $G_{r}=4$

Figure 5.12: Long sealed end SFD, linear coefficients vs. whirl amplitude ratio, results from MAXSFD, SQFDAMP and test data from [76] 


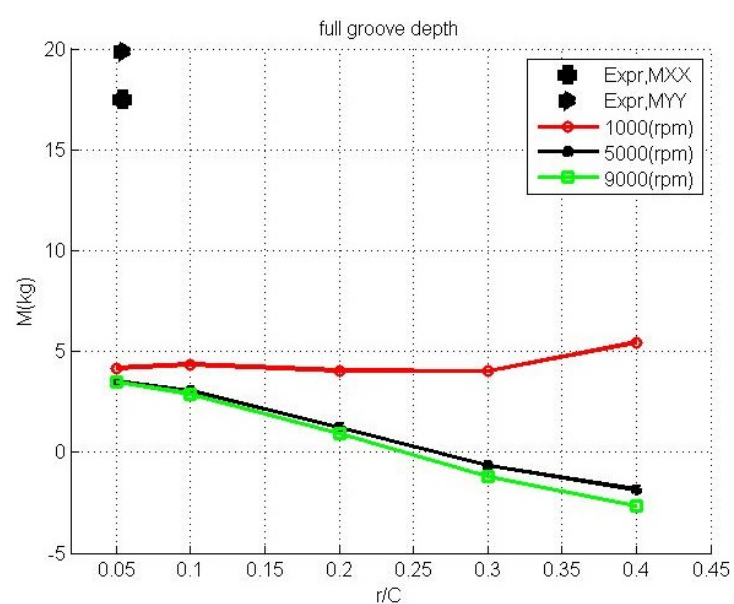

(a) Short, sealed ends, full groove

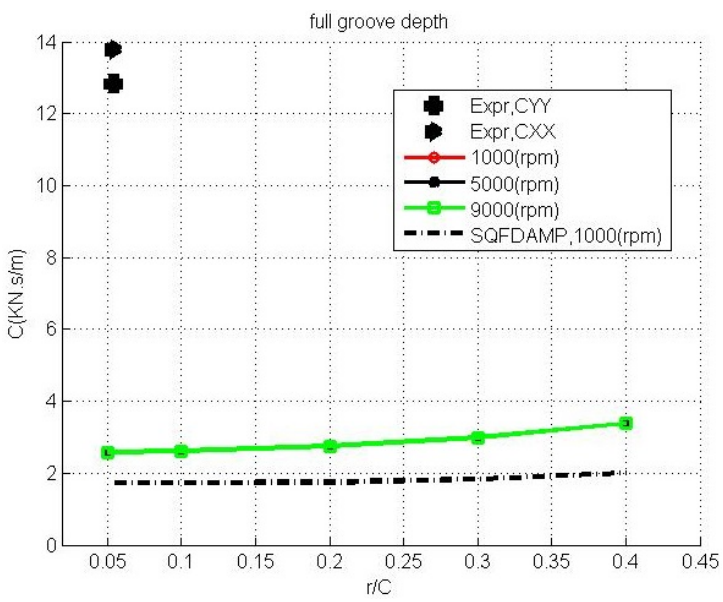

(c) Short, sealed ends, full groove

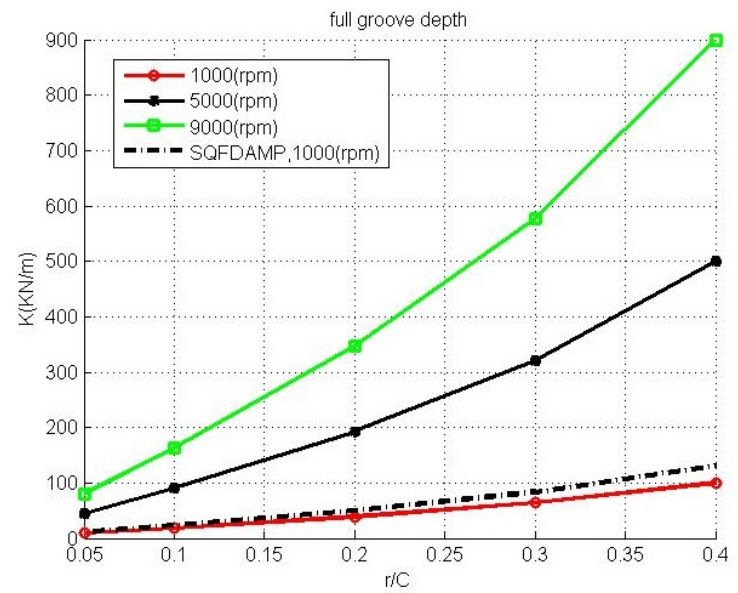

(e) Short, sealed ends, full groove

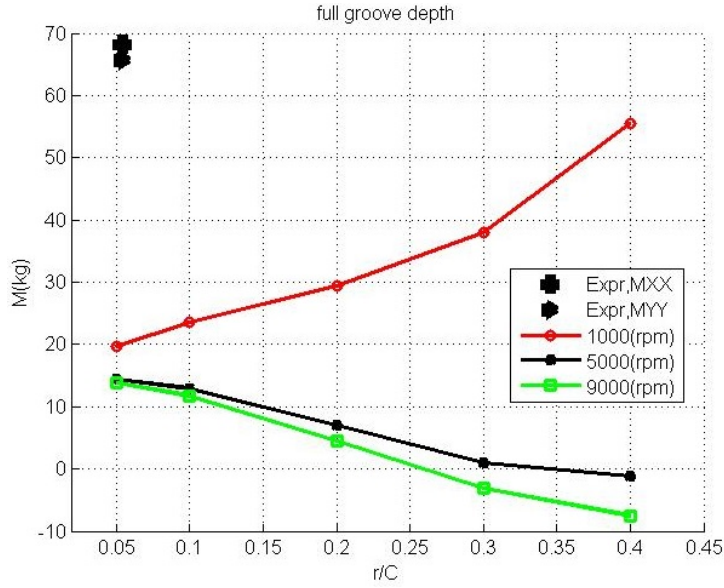

(b) Long, sealed ends, full groove

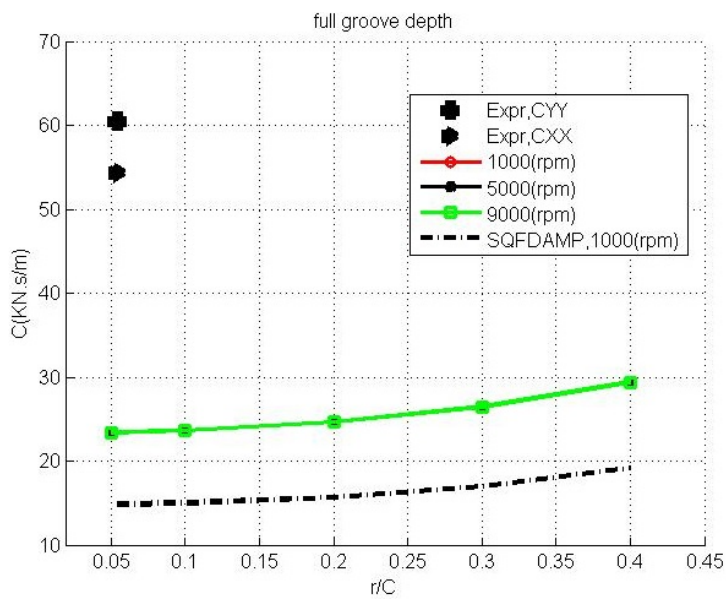

(d) Long, sealed ends, full groove

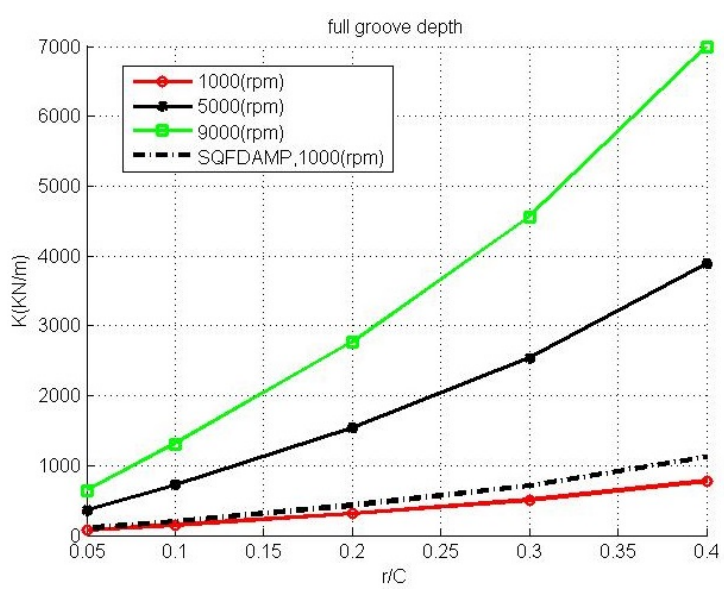

(f) Long, sealed ends, full groove

Figure 5.13: Short and long sealed end SFD, linear coefficients vs. whirl amplitude ratio with $d_{e f f}=9.5(\mathrm{~mm})$, results from MAXSFD, SQFDAMP and test data from [76] 
Table 5.2: Optimum effective groove depth ratio to predict linear coefficients

\begin{tabular}{c|c|c|c|c}
\hline \hline & short open end & long open end & short sealed end & long sealed end \\
\hline$M$ & 4.0 & 3.5 & 5 & 4 \\
\hline$C$ & 2.5 & 2 & 3.5 & 4 \\
\hline
\end{tabular}

damping values while overpredicting the mass value for all whirling speeds. On the other hand, a good correlation with mass values is achieved when $G_{r}=5.0$. However, $G_{r}=5.0$ underpredicts damping values by about $\sim 50 \%$. It can be observed that while both effective groove ratios show a close match with SQFDAMP stiffness trend, $G_{r}=5.0$ exhibits a closer correlation for $K$ values.

Examining the dynamic coefficients plots of the long sealed SFDs in Fig. (5.12) reveals a good correlation with $M$ and $C$ experimental data for both $G_{r}=3.0$ and $G_{r}=4.0$. For the tested long sealed SFD, the added mass values are three times larger compared to the open end long SFD. Reducing $G_{r}$ yields an increase in all added mass and damping values and a mild correlation improvement for the added mass coefficient. As expected, the damping values even when the full groove is considered equal to $d_{\text {eff }}$ are underpredicted by SQFDAMP due to its simplified underlying theories, especially for long SFD, see Fig. 5.13(d).

As seen, the added mass and damping values cannot be accurately predicted by just a single $d_{\text {eff }}$ value. Table (5.2) lists the optimum $G_{r}$ that bes predicts the $M$ or $C$ values. The $G_{r}$ that predicts $M$ is almost 1.5 times larger than the value which predicts $C$ the best for all the cases other than the long sealed SFD. However in the case of sealed end SFDs, the two values are nearly the same. The explanation for this lays in the way that the lubricant in the groove interacts with the journal surface. The damping coefficient $C$ represents the force tangential to the "CCO" exerted on the journal. And the lubricant in the depth of the groove makes a very small contribution 
to a force in this direction. On the other hand, $M$ represents the outward radial force acting on the journal due to the centrifugal acceleration of "CCO". Therefore, it can be reasoned that the lubricant body trapped in the depth of the groove would have more efficiently impact on the inertial force with a radial nature.

Figures $5.14 \& 5.15$ illustrate typical pressure profiles for open end and close end SFDs. The contribution of inertia effects on the pressure profile is also depicted. For both cases, it can be observed that the pressure pressure profile is level in the groove regions. Also by comparing the pressure values of cases with and without inertia contributions, it can be concluded that the squeeze velocity is the main pressure generating mechanism. These figures also depict the high resolution mesh areas close to the axial ends.

Figure (5.16) exhibits how the added mass terms are influenced by the selected $Q_{x}$ values. In most of the cases the secondary inertia effect acts toward reducing the added mass value and this effect is magnified as $Q_{x}$ increases. This reduction is most pronounced for the long sealed SFD. It can be observed that for some limited cases, with high whirl amplitude and low whirl speed, this trend is reverses; however, overall the secondary inertia effect can be assumed to be a relatively minor contribution toward the total SFD dynamics. 


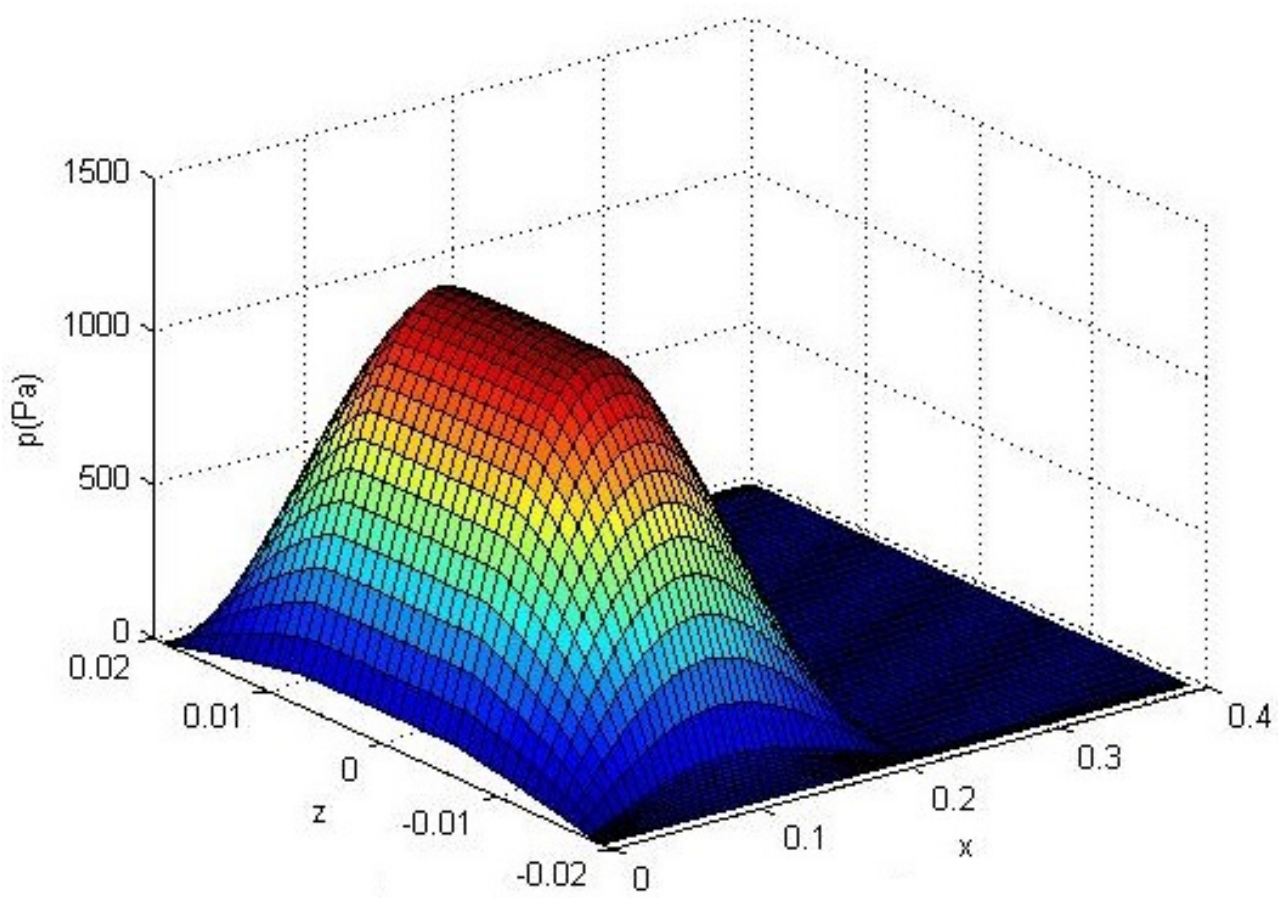

(a) Pressure profile without inertia contribution

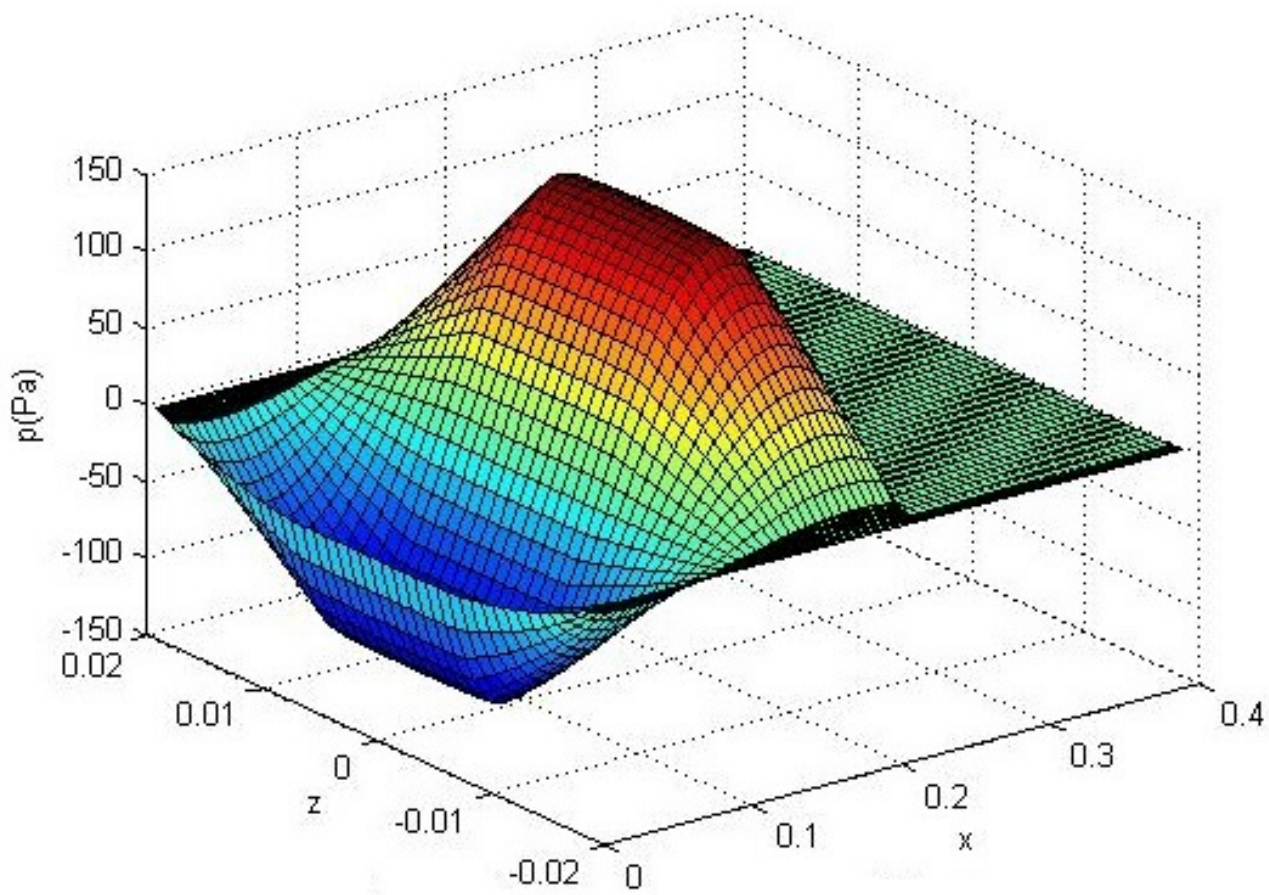

(b) Temporal inertia contribution in the pressure profile

Figure 5.14: Pressure profile of short open end SFD, $G_{r}=2.5, \frac{r}{c}=0.054, \Omega=$ $1000($ rpm $)$ 


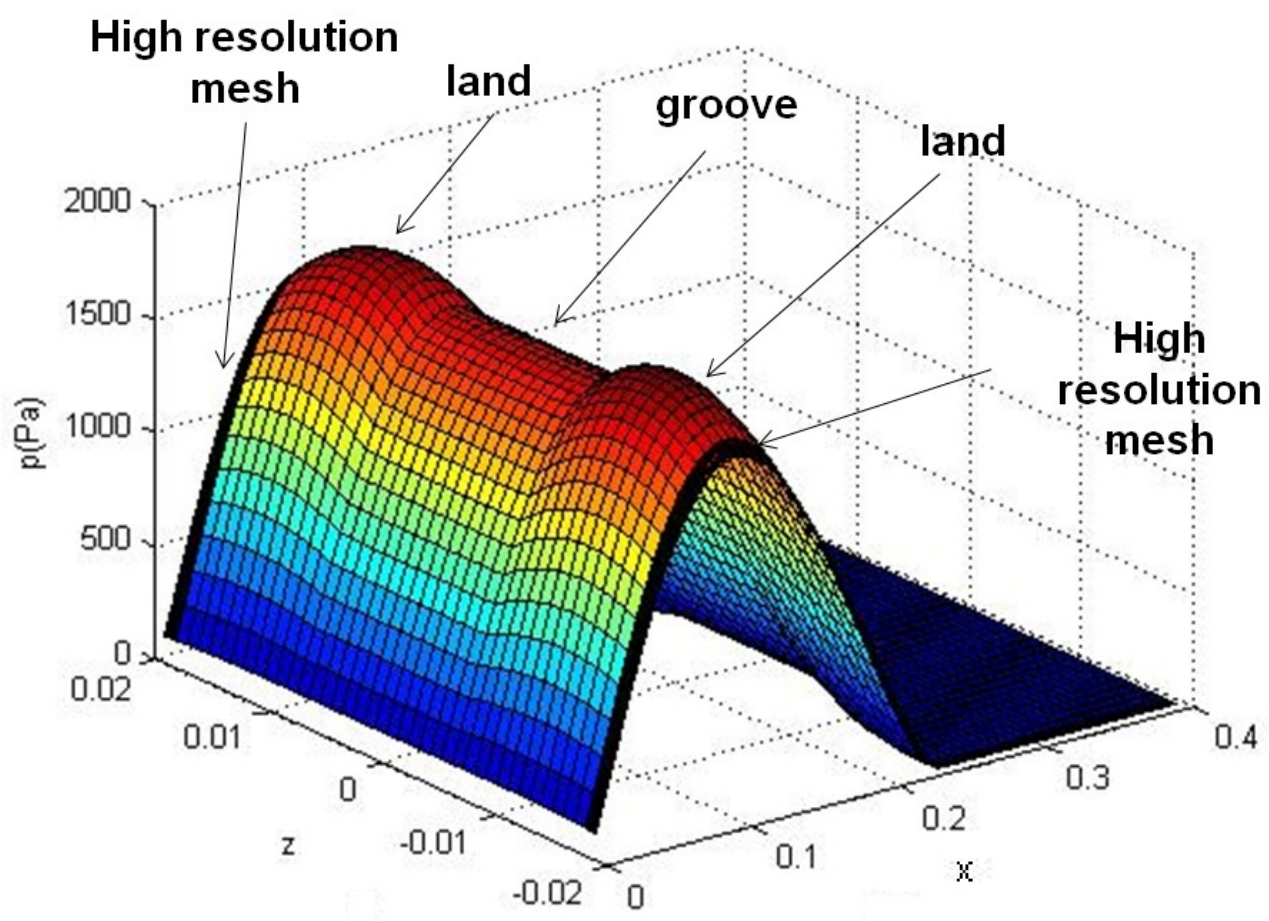

(a) Pressure profile without inertia contribution

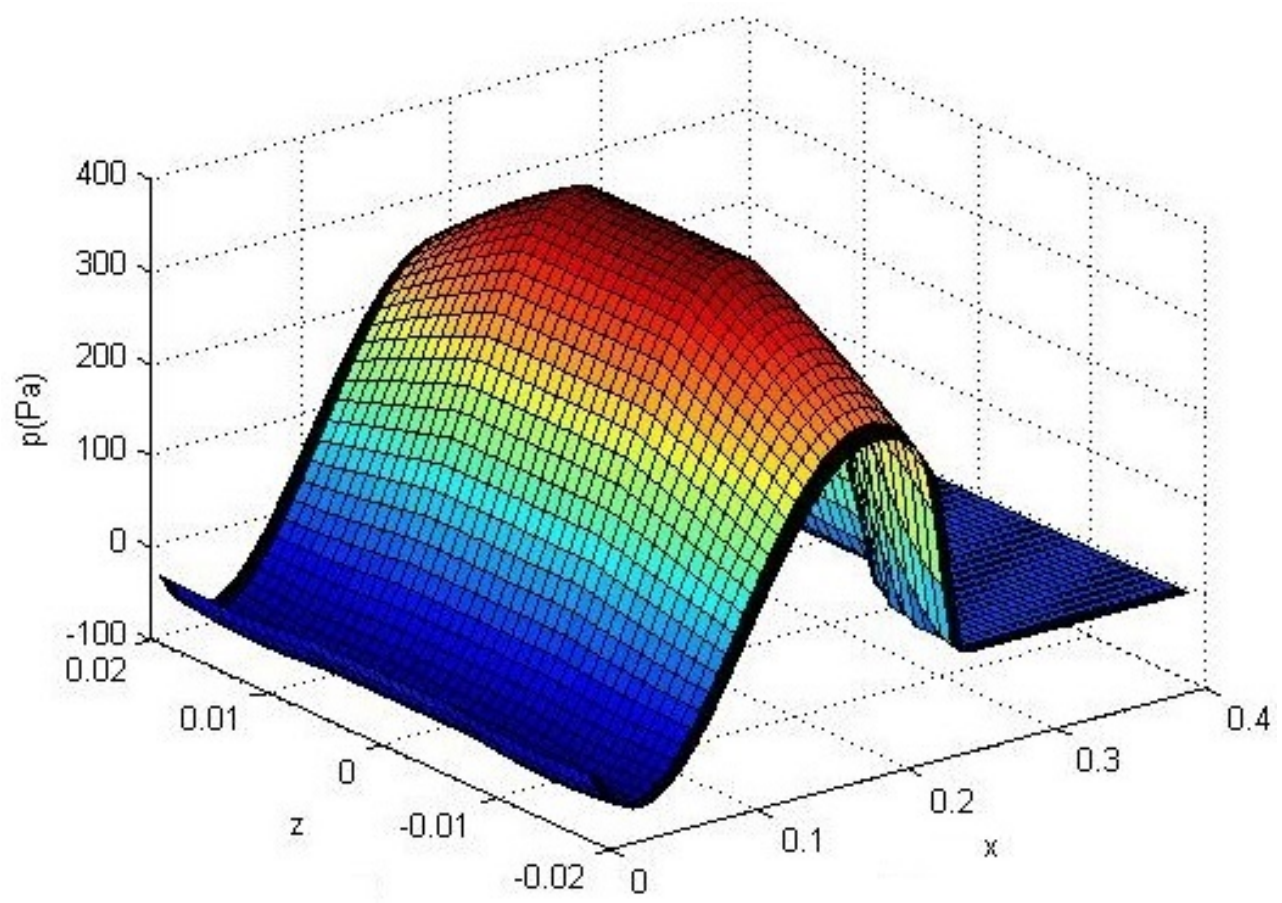

(b) Temporal inertia contribution in the pressure profile

Figure 5.15: Pressure profile of short sealed end SFD, $G_{r}=4, \frac{r}{c}=0.054, \Omega=$ $1000(\mathrm{rpm})$ 


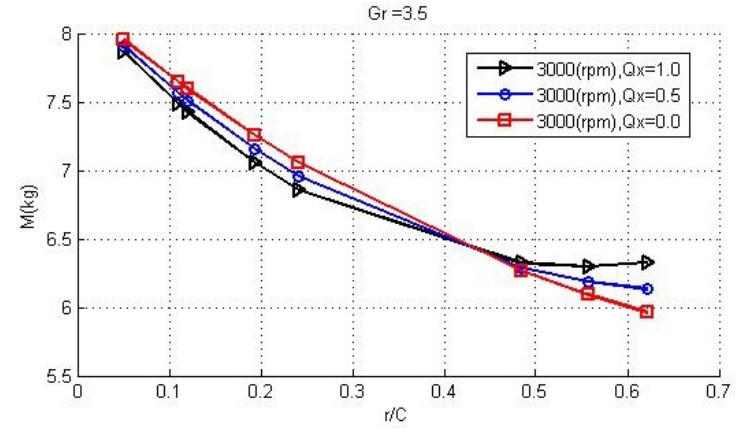

(a) Short open end SFD

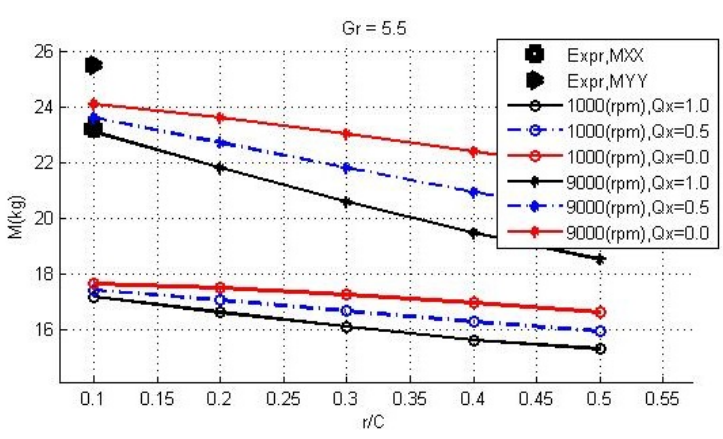

(c) Long open end SFD

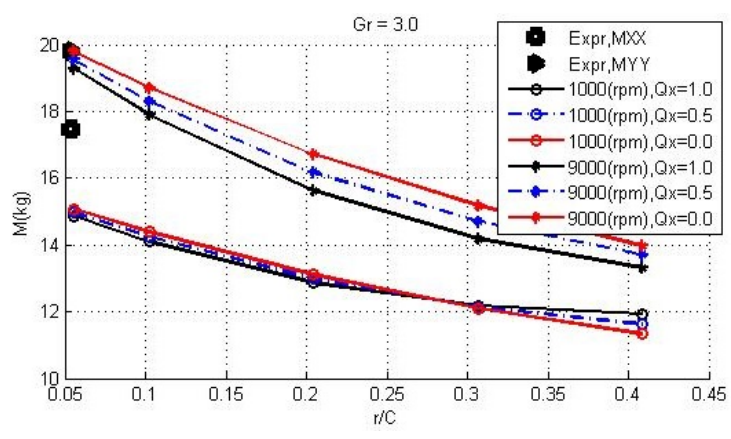

(b) Short sealed end SFD

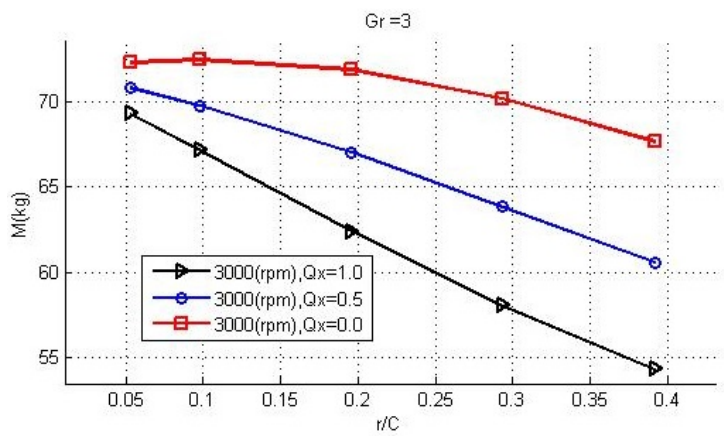

(d) Long sealed end SFD

Figure 5.16: Effect of secondary inertia models on the added mass coefficient for fixed $G_{r}$ values 


\section{Chapter 6}

\section{Conclusions and Recommendations}

This dissertation presented a comprehensive hydrodynamic model for incompressible fluid film lubrication. The previous chapters discussed the theoretical models, numerical methods and their applications to various bearing designs and operating conditions. The numerical results were extensively compared with published experimental data, CFD results and other modeling techniques. In general, this dissertation pursued three main goals.

I. Development of a comprehensive extended Reynolds equation for incompressible lubricants with the inclusion of an effective turbulence model and inertia effects.

II. Development of SLEEVEBRG, an isothermal hydrodynamic code, which is capable of analyzing full cylindrical sleeve bearings operating in extreme conditions with high $\mathbf{R e}$ and $\mathbf{R e}^{*}$. This code is best suited for water lubrication applications.

III. Development of MAXSFD, a hyrodynamic code designed to analyze various complex, realistic squeeze film damper designs and configurations in two dimensions. 
The work presented in this dissertation can be summarized as follows.

I. In chapter (1):

i. The assumptions leading to the development of thin film lubrication theory are discussed.

ii. An overview of water lubricated bearings and squeeze film dampers as the two main applications of the extended Reynolds equation is presented. The inadequacy of the traditional hydrodynamic theory to comprehensively address these two applications is elaborated on.

iii. A literature review of studies on extending the traditional Reynolds equation, and research on water bearings and squeeze film dampers is presented.

II. In chapter (2):

i. The full kinematic equations for journal bearings are derived, including the film geometry, velocity and acceleration expressions of an eccentric journal. This development is implemented in Cartesian coordinates (rather than polar coordinates) which assists in evaluating the magnitude of terms as well as the reduction and simplification of the extended Reynolds equation.

ii. The "general" form of the traditional Reynolds equation is developed for all types of lubricants and bearings. Then, the general form is reduced to the common traditional form applicable to incompressible lubricants.

iii. The extended Reynolds equation is developed which includes a modified turbulence model as well as both convective and temporal inertia terms. The turbulence model is a modified version of Constantinescu's approach. Constantinecu's model is adopted to take into account convective inertia. 
Temporal inertia is included in the most general form which contains both primary and secondary temporal inertia terms.

III. In chapter $(3)$ :

i. The Galerkin method is presented as the chosen numerical method to solve the extended Reynolds equation.

IV. In chapter (4):

i. With the application of a perturbation technique to the extended Reynolds equation, a zero order equation modeling the steady state operation of the bearing and auxiliary equations revealing the linear rotordynamic coefficients of the bearing are obtained. A rigid rotor stability analysis is developed. An auxiliary equation to produce added mass coefficients is presented. This equation takes into account both primary and secondary temporal inertia (for the first time) effects. To model secondary temporal inertia in radial bearings, a novel technique is proposed.

ii. The extended Reynolds equation theory is programmed in the computer code SLEEVEBRG, which is capable of handling full cylindrical sleeve bearings, especially those lubricated with low viscosity fluids.

iii. A single $80^{\circ}$ pad of a tilting pad bearing is analyzed. The results from this analysis are validated against CFD analysis. In addition, these results are used to tune Constantinescu's turbulence model utilized in SLEEVEBRG.

iv. SLEEVEBRG can be used in a limited way for single axial groove oil bearings and is used to analyze the Ferron et al. bearing. SLEEVEBRG's results are validated against experimental data, and are also compared with MAXBERG's results. It is concluded that SLEEVEBRG's results apply 
to the oil bearing best when the operating speed and the applied load is low. In addition, for the case of oil bearings, as expected, the added mass effect is shown to be negligible.

v. An analysis on a cylindrical water bearing is conducted by SLEEVEBRG for two general cases of zero and high axial supply pressures. It can be concluded that the supply pressure has a major effect on the equilibrium position of the journal center, and by suppressing cavitation, brings the bearing close to the long bearing operation assumption.

vi. Convective inertia increases the load capacity of the bearing and consequently decreases the eccentricity ratio, and accordingly, it acts toward increasing the principle stiffnesses, while the cross coupled stiffnesses are indifferent to its effects. Convective inertia has negligible effects on damping and added mass values. Overall, convective inertia demonstrates stabilizing effects.

vii. Huge added mass coefficients are predicted for water bearings which reach up to $90 \%$ of the shaft's physical mass. Secondary temporal inertia effects are found to be significant for lower Re and to produce negative added mass values. In general, the secondary temporal inertia effects act opposite to the primary temporal inertia and thereby act to reduce the added mass values.

viii. The added mass has destabilizing effects. However, the secondary temporal effect by reducing the added mass values, helps improve stability slightly.

ix. In general, cylindrical water bearings are prone to stability issues, especially at higher rotational speeds.

V. In chapter (5): 
i. The extended Reynolds equation is modified slightly to be applicable to SFDs and take into account hole effects.

ii. A computer code MAXSFD is developed to analyze SFDs by taking into account grooves, holes and end seals.

iii. The effective groove depth approach suggested by San Andres is adopted. This method takes a small fraction of the groove depth as the "squeeze" body of the lubricant that takes part in pressure generation in the groove region. By this means, the large dynamic pressures measured in the groove region are justified.

iv. MAXSFD uses a "whirl speed dependent" approach to find the linear coefficients of SFDs. It is shown that especially for the case of open-end SFDs, two distinct effective groove depths are required to predict the added mass and damping coefficients of SFDs correctly. The effective groove depths that predict damping better are often smaller than the ones that produce more accurate added mass coefficients. These two values are closer for sealed SFDs.

v. The effect of secondary temporal inertia is found to be negligible with respect to the added mass coefficients of SFDs.

This study answered many question, but it also opened many more questions and research areas. The author suggests the following as future work to extend this research area:

1. The extended Reynolds equation derived in this study targets cylindrical fixed geometry water bearings. This work can be extended to other configurations of water bearings with axial grooves, preloads and tilting pad bearings. 
2. The current version of MAXSFD assumes "CCO" for the operation of SFDs. In practice, off centered and non-circular orbits might occur. The next generations of MAXSFD can take into account more general orbits when calculating linear coefficients. 


\section{References}

[1] L. Barrett and E. J. Gunter. "Steady State and Transient Analysis of a Squeeze Film Damper Bearing for Rotor-Stability - A Manual for SQFDAMP”. June 1973 (revised June 1995), 100 p. MAE81/102.

[2] O. Reynolds. "On the Theory of Lubrication and Its Application to Mr. Beauchamp Tower's Experiments, Including an Experimental Determination of the Viscosity of Olive Oil". Philos. T. Roy. Soc. Lond., 177:157-234, 1886.

[3] J. Tichy and B. Bou-Said. "Hydrodynamic Lubrication and Bearing Behavior with Impulsive Loads". Tribology Transactions, 34(4):505-512, 1991.

[4] D. L. Cabrera, N. H. Woolley, D. R. Allanson, and Y. D. Tridimas. "Film pressure distribution in water-lubricated rubber journal bearings". Proceedings of the Institution of Mechanical Engineers, Part J: Journal of Engineering Tribology, 219(2):125-132, 2005.

[5] J. A. Tichy. "Effects of Fluid Inertia and Viscoelasticity on the One Dimensional Bearing". ASLE Trans., 27:164-167, 1984.

[6] J. A. Tichy. "Effects of Fluid Inertia and Viscoelasticity on Squeeze-Film Bearing Forces". ASLE Trans., 25:125-132, 1982. 
[7] J. A. Tichy and M. F. Modest. "A Simple Low Deborah Number Model for Unsteady Hydrodynamic Lubrication Including Fluid Inertia". Journal of Rheology, 24:829-845, 1980.

[8] T. M. Mulcahy. "Fluid Forces on Rods Vibrating in Finite Length Annular Regions". Trans. ASME J. Appl. Mech., pages 234-246, 1980. SFD.

[9] L. San Andres. "Effect of Fluid Inertia Effect on Squeeze Film Damper Force Response”. PhD thesis, Texas A\&M University, College Station, TX, 1985.

[10] L. San Andres and A. Delgado. "Identification of Force Coefficients in a Squeeze Film Damper with a Mechanical End Seal". Journal of Tribology, 129(3):660668, 2007. SFD.

[11] L. Della Pietra and G. Adiletta. "The Squeeze Film Damper Over Four Decades of Investigations. Part I: Characteristics and Operating Features". Shock and Vibration Digest, 34(1):3-26, 2002.

[12] A. Z. Szeri. "Fluid Film Lubrication Theory and Design". Cambridge University Press, Cambridge, UK, 1998.

[13] S. K. Kakoty and B. C. Majumdar. "Effect of Fluid Inertia on Stability of Oil Journal Bearings". Journal of Tribology, 122(4):741-745, February 1999.

[14] E. Reinhardt and J. W. Lund. "The Influence of Fluid Inertia on the Dynamic Properties of Journal Bearings". Journal of Lubrication Technology, 97:159-165, 1975.

[15] R. J. Grimm. "Squeezing Flows of Newtonian Liquid Films an Analysis Including Fluid Inertia". Applied Scientific Research, 32(2):149-166, 1976. 
[16] T. M. Mulcahy. "Fluid Forces on Rods Vibrating in Finite Length Annular Regions". Journal of Applied Mechanics, 47(2):234-240, June 1980.

[17] C. Brennen. "On the Flow in an Annulus Surrounding a Whirling Cylinder". Journal of Fluid Mechanics, 75(01):173-191, 1976.

[18] D. Wilcock. "Turbulence in High Speed Journal Bearings". Transactions of the ASME, 72:825-834, 1950.

[19] H. Schlichting and K. Gersten. "Boundary-Layer Theory". McGraw-Hill, New York, 1968.

[20] C. W. Ng. "Fluid Dynamic Foundation of Turbulent Lubrication Theory". ASLE Transactions, 7(4):311-321, January 1964.

[21] C. W. Ng and C. H. T. Pan. "A Linearized Turbulent Lubrication Theory". Journal of Basic Engineering, 87(3):675-682, September 1965.

[22] Jr. Elrod, H. G., and C. W. Ng. "A Theory for Turbulent Fluid Films and Its Application to Bearings". Journal of Lubrication Technology, 89(3):346-362, July 1967.

[23] G. G. Hirs. "A Bulk-Flow Theory for Turbulence in Lubricant Films". Journal of Lubrication Technology, 95(2):137-145, April 1973.

[24] N. Brunetiere. "A Modified Turbulence Model for Low Reynolds Numbers: Application to Hydrostatic Seals". Journal of Tribology, 127(1):130-140, February 2005.

[25] A.S. Monin and A. M. Yaglom. "Statistical Fluid Mechanics". MIT press, Massachusett, 1973. 
[26] Z. Safar and A. Z. Szeri. "Thermohydrodynamic Lubrication in Laminar and Turbulent Regimes". Journal of Lubrication Technology, 96:48-56, 1974.

[27] M. He, P. Allaire, and L. Barrett. "Thermohydrodynamic Modeling of LeadingEdge Groove Bearings Under Starvation Condition". Tribology Transactions, 48:362-369, 2005.

[28] N. Brunetiere and B. Tournerie. "The Effect of Inertia on Radial Flows in Application to Hydrostatic Seals". Journal of Tribology, 128(3):566-574, March 2006.

[29] N. Brunetiere and B. Tournerie. "Finite Element Solution of Inertia Influenced Flow in Thin Fluid Films". Journal of Tribology, 129(4):876-886, May 2007.

[30] T. Suganami and A. Z. Szeri. "A Thermodynamic Analyses of Journal Bearings". Journal of Lubrication Technology, 101:21-27, 1979.

[31] H. Hashimoto, S. Wada, and M. Sumitomo. "The Effects of Fluid Inertia Forces on the Dynamic Behavior of Short Journal Bearings in Superlaminar Flow Regime". Journal of Tribology, 110(3):539-545, July 1988.

[32] H. Hashimoto. "Viscoelastic Squeeze Film Characteristics With Inertia Effects Between Two Parallel Circular Plates Under Sinusoidal Motion". Journal of Tribology, 116(1):161-166, January 1994.

[33] J. F. Osterle, Y. T. Chou, and E. A. Saibel. "The Effect of Lubricant Inertia in Journal Bearing Lubrication". Journal of Applied Mechanics, 79:494-496, 1957. 
[34] V. N. Constantinescu. "On the Influence of Inertia Forces in Turbulent and Laminar Self-Acting Films". Journal of Lubrication Technology, 92(3):473-480, July 1970.

[35] V. N. Constantinescu and S. Galetuse. "On the Possibilities of Improving the Accuracy of the Evaluation of Inertia Forces in Laminar and Turbulent Films". Journal of Lubrication Technology, 96(1):69-77, January 1974.

[36] V. N. Constantinescu. "Basic Relationships in Turbulent Lubrication and Their Extension to Include Thermal Effects". Journal of Lubrication Technology, 95:147-154, 1973.

[37] V. N. Constantinescu and S. Galetuse. "Operating Characteristics of Journal Bearings in Turbulent Inertial Flow". Journal of Lubrication Technology, 104(2):173-179, April 1982.

[38] J. Frene and V. N. Constantinescu. "Non-Laminar Flow in Hydrodynamic Lubrication". In T.H.C. Childs G. Dalmaz Y. Berthier L. Flamand J.-M. Georges D. Dowson, C.M. Taylor and A.A. Lubrecht, editors, The Third Body Concept Interpretation of Tribological Phenomena, volume 31 of Tribology Series, pages 319 - 333. Elsevier, 1996.

[39] J. Frene, M. Arghir, and V. N. Constantinescu. "Combined Thin-Film and Navier-Stokes Analysis in High Reynolds Number Lubrication". Tribology International, 39:734-747, 2006.

[40] L. Bouard, M. Fillon, and J. Frene. "Comparison Between Three Turbulent Models Application to Thermohydrodynamic Performances of Tilting-Pad Journal Bearings". Tribology International, 29:11-18, 1996. 
[41] H. Xu and J. Zhu. "Research of Fluid Flow and Flow Transition Criteria from Laminar to Turbulent in a Journal Bearing". J. Xi'An Jiaotong University, $27: 7-14,1993$.

[42] M. He. "Thermoelastohydrodynamic Analysis of Fluid Film Journal Bearings". PhD thesis, University of Virginia, 2003.

[43] N. Wang, Q. Meng, P. Wang, T. Geng, and X. Yuan. "Experimental Research on Film Pressure Distribution of Water-Lubricated Rubber Bearing With Multiaxial Grooves". Journal of Fluids Engineering, 135:084501-084501, 2013.

[44] F. L. Duan. "Study on Water Lubrication Mechanism of Rubber Bearings". PhD thesis, Chongqing University, Chongqing, China, 2002.

[45] Q. H. Liu. "Numerical Calculation and Experimental Research on WaterLubrication Characteristics of Ship Stern Bearings". Master's thesis, Wuhan University of Technology, Wuhan, China, reference in wang water lubricated beairngs, 2008.

[46] R. Y. Wang, Z. L. Liu, and Y. Jin. "Research on Lubrication Properties of Water-Lubricated Rubber Bearing Based on Fluid Structure Interaction". Applied Mechanics and Materials, 79:159-165, 2011. reference 7 of wang2013.

[47] B. C. Majumdar, R. Pai, and D. J. Hargreaves. "Analysis of Water-Lubricated Journal Bearings with Multiple Axial Grooves". Proceedings of the Institution of Mechanical Engineers, Part J: Journal of Engineering Tribology, 218:135-146, 2004.

[48] R. S. Pai and R. Pai. "Stability of Four-Axial and Six-Axial Grooved WaterLubricated Journal Bearings Under Dynamic Load". Proceedings of the In- 
stitution of Mechanical Engineers, Part J: Journal of Engineering Tribology, 222(5):683-691, 2008.

[49] R. Pai, D. J. Hargreaves, and R. Brown. "Modelling of Fluid Flow in a 3-Axial Groove Water Bearing Using Computational Fluid Dynamics". 14th Australian fluid Mechanics conference. 2001 December.

[50] R. W. Armentrout, A. E. Reed, and M. He. "Investigation of Water-Lubricated Journal Bearing Using Conventional and CFD Approaches". In STLE Annual Conference, 2007.

[51] S. Dousti, J. Cao, A. Younan, P. Allaire, and T. Dimond. "Temporal and Convective Inertia Effects in Plain Journal Bearings with Eccentricity, Velocity and Acceleration". Journal of Tribology, 134(3):505-512, 2012.

[52] G. Adiletta and L. Della Pietra. "The Squeeze Film Damper Over Four Decades of Investigations. Part II: Rotordynamic Analyses with Rigid and Flexible Rotors". Shock and Vibration Digest, 34(2):97-126, 2002.

[53] E. J. Hahn. "Stability and Unbalance Response of Centrally Preloaded Rotors Mounted in Journal and Squeeze Film Bearings". ASME J. Lubrication Technology, 110:120-128, 1979.

[54] L. San Andres and J. M. Vance. "Force Coefficients for Open-Ended SqueezeFilm Dampers Executing Small-Amplitude Motions About an Off-Center Equilibrium Position". ASLE Transactions, 30(1):69-76, 1987.

[55] D. C. White. "Squeeze Film Journal Bearing". PhD thesis, Cambridge University, 1970. 
[56] A. El-Shafei and R. V. Eranki. "Dynamic Analysis of Squeeze Film Damper Supported Rotors Using Equivalent Linearization". Journal of Engineering for Gas Turbines and Power, 116(3):682-691, July 1994.

[57] and Kaplan J. A. Dousti, S., F. He, and P. E. Allaire. "Elastomer O-Rings as Centering Spring in Squeeze Film Dampers: Application to Turbochargers". In ASME Turbo Expo 2013: Turbine Technical Conference and Exposition. American Society of Mechanical Engineers, 2013.

[58] M. M. Dede, M. Dogan, and R. Holmes. "The Damping Capacity of a Sealed Squeeze Film Bearing". Journal of Tribology, 107(3):411-418, July 1985.

[59] E. Feder, P. N. Bansal, and A. Blanco. "Investigation of Squeeze Film Damper Forces Produced by Circular Centered Orbits". Journal of Engineering for Gas Turbines and Power, 100(1):15-21, January 1978.

[60] J. M. Vance and A. J. Kirton. "Experimental Measurement of the Dynamic Force Response of a Squeeze-Film Bearing Damper". Journal of Manufacturing Science and Engineering, 97(4):1282-1290, November 1975.

[61] A. El-Shafei and S. H. Crandall. "Fluid Inertia Forces in Squeeze Film Dampers". ASME, Rotating machinery and Vehicle Dynamics, 35:219-228, 1991.

[62] L. San Andres. "Effect of Fluid Inertia on Force Coefficients for the Long Squeeze Film Damper". Tribology Transactions, 31(3):370-375, 1988.

[63] J. A. Tichy. "The Effect of Fluid Inertia in Squeeze Film Damper Bearings: A Heuristic and Physical Description". ASME Paper 83-GT-177, 1983. 
[64] M. F. Modest and J. A. Tichy. "Squeeze Film Flow in Arbitrarily Shaped Journal Bearings Subject to Oscillations". Journal of Tribology, 100(3):323329, July 1978.

[65] A. Z. Szeri, A. A. Raimondi, and A. Giron-Duarte. "Linear Force Coefficients for Squeeze-Film Dampers". Journal of Lubrication Technology, 105(3):326334, July 1983.

[66] T. Qingchang, C. Ying, and W. Lyjiang. "Effect of a Circumferential Feeding Groove on Fluid Force in Short Squeeze Film Dampers". Tribol. Int., 30(6):409416, 1997.

[67] J. Zhang, J. Ellis, and J. B. Roberts. "Observations on the Nonlinear Fluid Forces in Short Cylindrical Squeeze Film Dampers". Journal of Tribology, 115(4):692-698, October 1993.

[68] A. Delgado-Marquez. " Linear Fluid Inertia Model for Improved Prediction of Force Coefficients in Grooved Squeeze Film Dampers and Grooved Oil Seal Rings". PhD thesis, Texas A\&M University, 2008.

[69] L. San Andres and J. M. Vance. "Experimental Measurement of the Dynamic Pressure Distribution in a Squeeze-Film Bearing Damper Executing CircularCentered Orbit". ASLE Transactions, 30(3):373-383, 1987.

[70] F. Zeidan and J. Vance. "Cavitation and Air Entrainment Effects on the Response of Squeeze Film Supported Rotors". Journal of Tribology, 112(2):347353, April 1990. 
[71] F. Y. Zeidan, L. San Andres, and J. M. Vance. "Design and Application of Squeeze Film Dampers in Rotating Machinery". In Proceedings of the 25th Turbomachinery Symposium, pages 169-188, 1996.

[72] G. L. Arauz. "Experimental Study of a Grooved Squeeze Film Damper". Master's thesis, Texas A\&M University, 1993.

[73] G. Arauz and L. San Andres. "Effect of a Circumferential Feeding Groove on the Dynamic Force Response of a Short Squeeze Film Damper". Journal of Tribology, 116(2):369-377, 1994.

[74] G. Arauz and L. San Andres. "Experimental Study on the Effect of a Circumferential Feeding Groove on the Dynamic Force Response of a Sealed Squeeze Film Damper". Journal of Tribology, 118(4):900-905, 1996. Experimental Study on the Effect of a Circumferential Feeding Groove on the Dynamic Force Response of a Sealed Squeeze Film Damper.

[75] S. Seshagiri. "Identification of Force Coefficients in Two Squeeze Film Dampers With a Central Groove”. PhD thesis, Texas A\&M University, 2011.

[76] L. San Andres and S. Seshagiri. "Damping and Inertia Coefficients for Two End Sealed Squeeze Film Dampers With a Central Groove: Measurements and Predictions". Journal of Engineering for Gas Turbines and Power, 135, 2013.

[77] L. P. Mahecha Mojica. "Experimental Dynamic Forced Performance of a Centrally Grooved, End Sealed Squeeze Film Damper". Master's thesis, Texas A\&M University, 2011.

[78] L. San Andres. "Damping and Inertia Coefficients for Two Open Ends Squeeze Film Dampers With a Central Groove: Measurements and Predictions". Jour- 
nal of Engineering for Gas Turbines and Power, 134(10):102506-102506, August 2012.

[79] M. D. Ramli, J. B. Roberts, and J. Ellis. "Determination of Squeeze-Film Dynamic Coefficients From Experimental Transient Data". Journal of Tribology, 109(1):155-163, January 1987.

[80] L. San Andres. "Force Coefficients For a Large Clearance Open Ends Squeeze Film Damper with a Central Feed Groove: Experiments and Predictions". Tribology International, 71(0):17 - 25, 2014.

[81] R. A. Marmol and J. M. Vance. "Squeeze Film Damper Characteristics for Gas Turbine Engines". Journal of Mechanical Design, 100(1):139-146, January 1978. SFD.

[82] F. A. Rodrigues, F Thouverez, and L. Jezequel. "Interaction of Squeeze Film Dampers and Hole Feed Systems and Its Influence on the Dynamics of a Jeffcott Rotor". International Journal of Rotating Machinery, 10(3):163-174, 2004.

[83] F. He. "Forced Response and Stability of Flexible Rotor-Bearing Systems with Squeeze Film Dampers". PhD thesis, University of Virginia, 2013.

[84] P. Y. P. Chen and E. J. Hahn. "Pressure Distribution in Squeeze Film Dampers with Oil Hole Feed". Proceedings of the Institution of Mechanical Engineers, Part J: Journal of Engineering Tribology, 208(2):105-112, 1994.

[85] J. R. Bunch and J. Hopcroft. "Triangular Factorization and Inversion by Fast Matrix Multiplication". Mathematics of Computation, 28:231236, 1974.

[86] R. D. Cook, D. S. Malkus, M. E. Plesha, and R. J. Witt. "Concepts and Applications of Finite Element Analysis”. John Wiley \& Sons, 2007. 
[87] P. Klit and J. W. Lund. "Calculation of the Dynamic Coefficients of a Journal Bearing, Using a Variational Approach". Journal of Tribology, 108:421-425, 1986.

[88] L. Barrett. "Lubrication Theory Course Notes". University of Virginia, 1997.

[89] L. San Andres and A. Delgado. "A Novel Bulk-Flow Model for Improved Predictions of Force Coefficients in Grooved Oil Seals Operating Eccentrically". Journal of Engineering for Gas Turbines and Power, 134, 2011.

[90] S. A. Gandjalikhan Nassab. "Inertia Effect on the Thermohydrodynamic Characteristics of Journal Bearings". Proceedings of the Institution of Mechanical Engineers, Part J: Journal of Engineering Tribology, 219(6):459-467, 2005.

[91] R. X. Dai, Q. Dong, and A. Z. Szeri. "Approximations in Hydrodynamic Lubrication". Journal of Tribology, 114(1):14-25, January 1992.

[92] R. C. DiPrima and J. T. Stuart. "Flow Between Eccentric Rotating Cylinders". Journal of Tribology, 94(3):266-274, July 1972.

[93] J. Ferron, J. Frene, and R. Boncompain. "A Study of the Thermohydrodynamic Performanceof a Plain Journal Bearing Comparison Between Theory and Experiments". Journal of Lubrication Technology, 105:422-428, 1983.

[94] A. Cameron and C. M. Mc Ettles. "Basic Lubrication Theory". E. Horwood., 1976.

[95] L. San Andres and A. Delgado. "Squeeze Film Damper With a Mechanical End Seal: Experimental Force Coefficients Derived From Circular Centered Orbits". Journal of Engineering for Gas Turbines and Power, 130(4):042505, April 2008. SFD. 
[96] A. A. Younan, J. Cao, T. W. Dimond, and P. E. Allaire. "Nonlinear Analysis of Squeeze Film Damper with Entrained Air in Rotordynamic Systems". Tribology Transactions, 54(1):132-144, 2010.

[97] S. Dousti, T. W. Dimond, P. E. Allaire, and H. E. Wood. "Time Transient Analysis of Horizontal Rigid Rotor Supported With O-Ring Sealed Squeeze Film Damper". ASME 2013 International Mechanical Engineering Congress and Exposition, ASME, 2013, November.

[98] M. He and P. Allaire. "The Manual for HYDROBRG". University of Virginia, 2004 .

[99] E. J. Gunter. "Dynamic Stability of Rotor-Bearing Systems". National Aeronautics and Space Administration, 1966.

[100] G. T. Mase and G. E. Mase. "Continuum Mechanics for Engineers". 2010. 


\section{Appendix A}

\section{Perturbation of the Extended Reynolds Equation}

The position perturbation, $\Delta \zeta$, of the extended Reynolds equation (Eq. 2.60) results in the film thickness variation. Similarly, the velocity and acceleration perturbations yield perturbations of the time derivatives of film thickness. The variation in film thickness and it derivatives consequently perturbs the pressure. The variable $\zeta$ rep-

resents the horizontal, $X$, or vertical, $Y$, displacements shown in Fig. 2.2). We have

$$
\begin{aligned}
& h=h_{o}+\Delta h=h_{o}+h_{, \zeta} \Delta \zeta \\
& \frac{\partial h}{\partial t}=\frac{\partial h_{o}}{\partial t}+\Delta \frac{\partial h}{\partial t}=\frac{\partial h_{o}}{\partial t}+\left(\frac{\partial h}{\partial t}\right)_{, \dot{\zeta}} \Delta \dot{\zeta} \\
& \frac{\partial^{2} h}{\partial t^{2}}=\frac{\partial^{2} h_{o}}{\partial t^{2}}+\Delta \frac{\partial^{2} h}{\partial t^{2}}=\frac{\partial^{2} h_{o}}{\partial t^{2}}+\left(\frac{\partial^{2} h}{\partial t^{2}}\right)_{, \ddot{\zeta}} \Delta \ddot{\zeta} \\
& p=p_{o}+p_{, \zeta} \Delta \zeta+p_{, \dot{\zeta}} \Delta \dot{\zeta}+p_{, \ddot{\zeta}} \Delta \ddot{\zeta}
\end{aligned}
$$

Substituting Eqs. A.1 A.4 into the nonlinear pressure terms of Eq. 2.60, or equivalently expanding them using the Maclaurin formulation about the equilibrium position 
up to the linear terms yields

$$
\begin{aligned}
h^{3} \frac{\partial p}{\partial x} & =\left[h_{o}+\frac{\partial h}{\partial \zeta} \Delta \zeta\right]^{3}\left[\frac{\partial p_{o}}{\partial x}+\frac{\partial}{\partial x}\left(\frac{\partial p}{\partial \zeta}\right) \Delta \zeta\right] \\
& \approx h_{o}^{3} \frac{\partial p_{o}}{\partial x}+\left[3 h_{o}^{2} \frac{\partial p_{o}}{\partial x} \frac{\partial h}{\partial \zeta}+h_{o}^{3} \frac{\partial}{\partial x}\left(\frac{\partial p}{\partial \zeta}\right)\right] \Delta \zeta \\
h^{3} \frac{\partial p}{\partial z} & =\left[h_{o}+\frac{\partial h}{\partial \zeta} \Delta \zeta\right]^{3}\left[\frac{\partial p_{o}}{\partial z}+\frac{\partial}{\partial z}\left(\frac{\partial p}{\partial \zeta}\right) \Delta \zeta\right] \\
& \approx h_{o}^{3} \frac{\partial p_{o}}{\partial z}+\left[3 h_{o}^{2} \frac{\partial p_{o}}{\partial z} \frac{\partial h}{\partial \zeta}+h_{o}^{3} \frac{\partial}{\partial z}\left(\frac{\partial p}{\partial \zeta}\right)\right] \Delta \zeta
\end{aligned}
$$

Similarly, the shear term is

$$
\frac{U_{2}+U_{1}}{2} \frac{\partial}{\partial x}\left[h_{o}+\frac{\partial h}{\partial \zeta} \Delta \zeta\right]=\frac{U_{2}+U_{1}}{2} \frac{\partial h_{o}}{\partial x}+\frac{U_{2}+U_{1}}{2} \frac{\partial}{\partial x}\left(\frac{\partial h}{\partial \zeta}\right) \Delta \zeta
$$

As mentioned before, in deriving the perturbation of the convective inertia, $I_{c}$, and correction terms, $C$, it is assumed that the displacement perturbation is so small that $U_{p}$ and $W_{p}$ do not change significantly, thus, they are considered invariant and equal to the values calculated at the equilibrium position. The direct and cross-coupled stiffness coefficients are calculated by integrating the perturbed pressure solution. We have

$$
\begin{aligned}
& I_{c} \approx I_{c o}+\frac{\partial I_{c}}{\partial \zeta} \Delta \zeta \\
& C \approx C_{o}+\frac{\partial C}{\partial \zeta} \Delta \zeta
\end{aligned}
$$

where

$$
\begin{aligned}
\frac{\partial I_{c}}{\partial \zeta}= & -\frac{\rho}{\mu} \frac{\partial}{\partial \zeta}\left[\frac{1}{k_{x}} \frac{\partial h^{2}}{\partial x} \frac{\partial I_{x x}}{\partial x}+\frac{1}{k_{x}} \frac{\partial h^{2}}{\partial x} \frac{\partial I_{x z}}{\partial z}\right. \\
& \left.+\frac{h^{2}}{k_{x}} \frac{\partial^{2} I_{x x}}{\partial x^{2}}+h^{2} \frac{\partial^{2} I_{x z}}{\partial x \partial z}\left(\frac{1}{k_{x}}+\frac{1}{k_{z}}\right)+\frac{h^{2}}{k_{z}} \frac{\partial^{2} I_{z z}}{\partial z^{2}}\right]
\end{aligned}
$$


To calculate Eq. A.10), term by term derivation is conducted as

$$
\begin{aligned}
\frac{\partial}{\partial \zeta}\left(\frac{\partial h^{2}}{\partial x} \frac{\partial I_{x x}}{\partial x}\right) & =2 \frac{\partial h}{\partial \zeta} \frac{\partial h}{\partial x} \frac{\partial I_{x x}}{\partial x}+2 h \frac{\partial^{2} h}{\partial x \partial \zeta} \frac{\partial I_{x x}}{\partial x}+2 h \frac{\partial h}{\partial x} \frac{\partial^{2} I_{x x}}{\partial x \partial \zeta} \\
\frac{\partial}{\partial \zeta}\left(\frac{\partial h^{2}}{\partial x} \frac{\partial I_{x z}}{\partial z}\right) & =2 \frac{\partial h}{\partial \zeta} \frac{\partial h}{\partial x} \frac{\partial I_{x z}}{\partial z}+2 h \frac{\partial^{2} h}{\partial x \partial \zeta} \frac{\partial I_{x z}}{\partial z}+2 h \frac{\partial h}{\partial x} \frac{\partial^{2} I_{x z}}{\partial z \partial \zeta} \\
\frac{\partial}{\partial \zeta}\left(h^{2} \frac{\partial^{2} I_{x x}}{\partial x^{2}}\right) & =2 h \frac{\partial h}{\partial \zeta} \frac{\partial^{2} I_{x x}}{\partial x^{2}}+h^{2} \frac{\partial^{3} I_{x x}}{\partial \zeta \partial x^{2}} \\
\frac{\partial}{\partial \zeta}\left(h^{2} \frac{\partial^{2} I_{x z}}{\partial x \partial z}\right) & =2 h \frac{\partial h}{\partial \zeta} \frac{\partial^{2} I_{x z}}{\partial x \partial z}+h^{2} \frac{\partial^{3} I_{x z}}{\partial \zeta \partial x \partial z} \\
\frac{\partial}{\partial \zeta}\left(h^{2} \frac{\partial^{2} I_{z z}}{\partial z^{2}}\right) & =2 h \frac{\partial h}{\partial \zeta} \frac{\partial^{2} I_{z z}}{\partial z^{2}}+2 \alpha h^{2}\left(\frac{\partial W_{p}}{\partial z}\right)^{2} \frac{\partial h}{\partial \zeta}+2 \alpha W_{p} \frac{\partial^{2} W_{p}}{\partial z^{2}} \frac{\partial h}{\partial \zeta}
\end{aligned}
$$

Taking the displacement derivatives of Eqs. 2.71 2.77) results in

$$
\begin{aligned}
\frac{\partial}{\partial \zeta} \frac{\partial I_{x x}}{\partial x}= & \frac{I_{x x}}{h} \frac{\partial^{2} h}{\partial x \partial \zeta} \\
\frac{\partial}{\partial \zeta} \frac{\partial I_{x z}}{\partial z}= & \alpha \frac{\partial U_{p}}{\partial z} W_{p} \frac{\partial h}{\partial \zeta}+\alpha \frac{\partial W_{p}}{\partial z} U_{p} \frac{\partial h}{\partial \zeta}+\frac{1}{2}\left(U_{1}+U_{2}\right) \frac{\partial W_{p}}{\partial z} \frac{\partial h}{\partial \zeta} \\
\frac{\partial}{\partial \zeta} \frac{\partial^{2} I_{x x}}{\partial x^{2}}= & \frac{\partial^{2} I_{x x}}{\partial x \partial \zeta} \frac{1}{h} \frac{\partial h}{\partial x}-\frac{\partial I_{x x}}{\partial x} \frac{1}{h^{2}} \frac{\partial h}{\partial \zeta} \frac{\partial h}{\partial x}+\frac{\partial I_{x x}}{\partial x} \frac{1}{h} \frac{\partial^{2} h}{\partial \zeta \partial x} \\
& +\frac{I_{x x}}{h} \frac{\partial^{3} h}{\partial \zeta \partial x^{2}}+\frac{I_{x x}}{h^{3}} \frac{\partial h}{\partial \zeta}\left(\frac{\partial h}{\partial x}\right)^{2}-2 \frac{I_{x x}}{h^{2}} \frac{\partial h}{\partial x} \frac{\partial^{2} h}{\partial \zeta \partial x} \\
\frac{\partial}{\partial \zeta} \frac{\partial^{2} I_{x z}}{\partial x \partial z}= & \frac{\partial I_{x z}}{\partial z} \frac{1}{h} \frac{\partial^{2} h}{\partial \zeta \partial x}
\end{aligned}
$$

In Eq. A.9, the derivative term is

$$
\frac{\partial C}{\partial \zeta}=\frac{\partial}{\partial \zeta}\left\{\frac{\delta \rho}{k_{x} \mu} q_{x}^{2} \frac{\partial^{2} h}{\partial x^{2}}+\frac{2 \delta \rho}{k_{x} \mu} q_{x} \frac{\partial q_{x}}{\partial x} \frac{\partial h}{\partial x}\right\}
$$


which by taking the derivatives of Eq. (2.44) can be represented more concisely as

$$
\begin{aligned}
\frac{\partial C}{\partial \zeta}=\frac{\delta \rho}{k_{x} \mu} & \left\{2 \frac{q_{x}^{2}}{h} \frac{\partial h}{\partial \zeta} \frac{\partial^{2} h}{\partial x^{2}}+q_{x}^{2} \frac{\partial^{3} h}{\partial \zeta \partial x^{2}}+2 \frac{q_{x}}{h} \frac{\partial q_{x}}{\partial x} \frac{\partial h}{\partial \zeta} \frac{\partial h}{\partial x}+\right. \\
& \left.2 \frac{q_{x}^{2}}{h} \frac{\partial^{2} h}{\partial \zeta \partial x} \frac{\partial h}{\partial x}+2 q_{x} \frac{\partial U_{p}}{\partial x} \frac{\partial h}{\partial \zeta} \frac{\partial h}{\partial x}+2 q_{x} \frac{\partial q_{x}}{\partial x} \frac{\partial^{2} h}{\partial \zeta \partial x}\right\}
\end{aligned}
$$

The contribution of the zeroth order Reynolds equation to the first order equation reveals two terms that need to be expanded as follows for programming purposes

$$
\begin{aligned}
& \frac{\partial}{\partial x}\left\{\frac{3 h_{o}^{2}}{k_{x} \mu} \frac{\partial p_{o}}{\partial x} \frac{\partial h}{\partial \zeta}\right\}=\frac{6 h_{o}}{k_{x} \mu} \frac{\partial p_{o}}{\partial x} \frac{\partial h}{\partial x} \frac{\partial h}{\partial \zeta}+\frac{3 h_{o}^{2}}{k_{x} \mu} \frac{\partial^{2} p_{o}}{\partial x^{2}} \frac{\partial h}{\partial \zeta}+\frac{3 h_{o}^{2}}{k_{x} \mu} \frac{\partial p_{o}}{\partial x} \frac{\partial^{2} h}{\partial x \partial \zeta} \\
& \frac{\partial}{\partial z}\left\{\frac{3 h_{o}^{2}}{k_{z} \mu} \frac{\partial p_{o}}{\partial z} \frac{\partial h}{\partial \zeta}\right\}=\frac{3 h_{o}^{2}}{k_{z} \mu} \frac{\partial^{2} p_{o}}{\partial z^{2}} \frac{\partial h}{\partial \zeta}
\end{aligned}
$$




\section{Appendix B}

\section{Derivation of the General}

\section{Reynolds Equation For An}

\section{Isothermal Compressible Fluid}

In this section, the general form of the traditional Reynolds equation for isothermal compressible fluids is derived. This development closely follows the derivation of Navier-Stokes equations presented in [99] by Gunter. The derivation begins with considering the forces acting on a small volume element $d \tau$ shown in Fig. (B.1). The equations of motion for this volume can be expressed as

$$
\iiint_{v} \rho \frac{D \overrightarrow{\mathbf{u}}}{D t} d \tau=\iint_{s} \overrightarrow{\mathbf{T}} \cdot \overrightarrow{\mathbf{n}} d s+\iiint_{v} \overrightarrow{\mathbf{F}} d \tau
$$

where $\frac{D}{D t}$ is the material derivative defined as

$$
\frac{D}{D t}=\frac{\partial}{\partial t}+u \frac{\partial}{\partial x}+v \frac{\partial}{\partial y}+w \frac{\partial}{\partial z}
$$




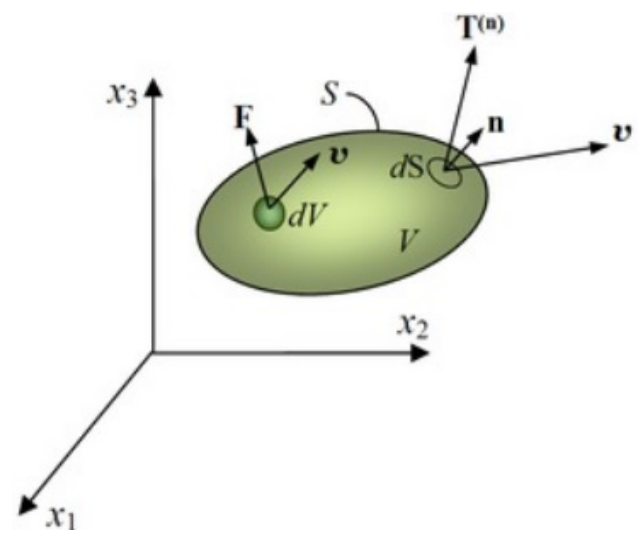

Figure B.1: Force acting on a small volume element

$\overrightarrow{\mathbf{F}}$ is the volumetric body force vector and $\overrightarrow{\mathbf{T}}$ is the traction vector defined as

$$
\overrightarrow{\mathbf{T}}=\tilde{\sigma} \cdot \overrightarrow{\mathbf{n}}
$$

where $\tilde{\sigma}$ is the stress tensor and $\overrightarrow{\mathbf{n}}$ is the normal surface vector, as shown in Fig. (B.1). By employing Gauss's theorem, Eq. B.1 becomes

$$
\iiint_{v}\left[\rho \frac{D u_{i}}{D t}-\frac{\partial \sigma_{i j}}{\partial X_{j}}-F_{i}\right] d \tau=0
$$

Since the volume of integration is arbitrary, then

$$
\rho \frac{D u_{i}}{D t}=\frac{\partial \sigma_{i j}}{\partial X_{j}}+F_{i} ; \quad i=1,2,3
$$

Let

$$
\sigma_{i j}=-p \delta_{i j}+\tau_{i j}
$$

where $\delta$ is Kronecker delta and $\tau_{i j}$ s are viscous shear stresses.

If the fluid is assumed to be Newtonian, then the viscous shear stresses are linearly 
related to the rate of shear strain, $\epsilon$. This is represented by

$$
\tau_{i j}=C_{i j k l} \epsilon_{k l}
$$

If the fluid is also isotropic [100], then the fourth-order tensor $C_{i j k l}$ is symmetric and invariant under coordinate transformation and can be expressed by

$$
C_{i j k l}=\lambda \delta_{i j} \delta_{k l}+\mu\left[\delta_{i k} \delta_{j l}+\delta_{i l} \delta_{j k}\right]+\gamma\left[\delta_{i k} \delta_{j l}-\delta_{i l} \delta_{j k}\right]
$$

where $\lambda, \mu, \gamma$ are Lamé constants. Hence the stress-strain rate for an isotropic Newtonian fluid is given by

$$
\tau_{i j}=\lambda \delta_{i j} \Delta+2 \mu \epsilon_{i j}
$$

where the symmetric strain rate tensor is defined as $\epsilon_{i j}=\frac{1}{2}\left(u_{i, j}+u_{j, i}\right)$, and $\Delta=$ $\epsilon_{i i}=u_{i, i}$ is dilatation. Contraction of Eq. (B.9) results in

$$
\tau_{i i}=(3 \lambda+2 \mu) \Delta
$$

In the case of an incompressible fluid where the dilatation is negligible, the sum of the viscous normal stresses $\tau_{i i}$ is zero, thus, the constitutive equation for such a fluid becomes

$$
\sigma_{i j}=-p \delta_{i j}+2 \mu \epsilon_{i j}
$$

and based on the pressure definition[100], we also have

$$
p=-\frac{1}{3} \sigma_{i i}
$$

Therefore, for an incompressible viscous fluid, the pressure $p$ has the meaning of 
the mean normal compressive stress. The value of $p$ does not depend explicitly on any kinematic quantities; its value is indeterminate as far as the fluid's mechanical behavior is concerned. In other words, since the fluid is incompressible, one can superimpose any pressure to the fluid without affecting its mechanical behavior. Thus, the pressure in an incompressible fluid is often known constitutively as the "indeterminate pressure". In any given problem with prescribed boundary condition(s) for the pressure, the pressure field is determinate.

For a compressible fluid, on the other hand, in general $\Delta \neq 0$. It is clear that the "pressure" in this case does not have the meaning of the mean normal compressive stress as was true for the incompressible case. However, if we assume that $\tau_{i i}$ will be zero even for a compressible medium, then based on Eq. B.10

$$
3 \lambda+2 \mu=0, \quad \lambda=-\frac{2}{3} \mu
$$

This is known as Stokes approximation and discards one of the Lamé constants from the governing equations of motion. This approximation has been shown to be true only for the case of monatomic gases, but usually results in only higher order deviations for most gases at normal temperature and pressure. This assumption is invalid in regions where large pressure or velocity gradients exist. As an example, the assumption breaks down in the immediate vicinity of a supply orifice to an externally pressurized bearing if a shock wave occurs. Substituting Eq. (B.13) into Eq. (B.9), Eq. (B.6) can be written as

$$
\sigma_{i j}=-p \delta_{i j}+\mu\left(-\frac{2}{3} \delta_{i j} u_{k, k}+u_{i, j}+u_{j, i}\right)
$$


And the equations of motion become

$$
\rho \frac{D u_{i}}{D t}=-\frac{\partial p}{\partial X_{i}}+F_{i}+\frac{\partial}{\partial X_{j}}\left[\mu\left(-\frac{2}{3} \delta_{i j} u_{k, k}+u_{i, j}+u_{j, i}\right)\right]
$$

Which is the well-known Navier-Stokes equation set. If the viscosity $\mu$ is not a function of the coordinate $X^{\uparrow}$, then Eq. B.15) reduces to

$$
\rho \frac{D u_{i}}{D t}=-\frac{\partial p}{\partial X_{i}}+F_{i}+\mu\left(\frac{1}{3} u_{j, i j}+u_{i, j j}\right)
$$

If the body forces $F_{i}$ are zero (which is the case for thin film lubrication Fig. (B.2)), then Eq. B.16 relates the inertia forces to the rates of change of the hydrostatic pressure and viscous shear stresses. A significant assumption in the formulation of a lubrication problem is that the flow is laminar. This is possible only if the inertia terms of the left-hand side of Eq. (B.16) are small in comparison to the viscous shear forces. The reduced Reynolds number represents the ratio of the inertia to the viscous forces

$$
\mathbf{R e}^{*}=\frac{\text { Inertia forces }}{\text { Viscous forces }} \approx \frac{\rho \frac{U^{2}}{L}}{\mu \frac{U}{h^{2}}}=\frac{U L}{\nu}\left(\frac{h}{L}\right)^{2}
$$

and if the reduced Reynolds number $\mathbf{R e}^{*}$ is much less than 1

$$
\operatorname{Re}^{*} \ll 1
$$

\footnotetext{
${ }^{1}$ Care must be taken that in bearings in general viscosity varies due to thermal gradient. However, this is a secondary effect and for an isothermal condition, viscosity is constant. To capture the viscosity variations, iterations are conducted in which energy equation is solved, the temperature profile is calculated, and the viscosity is updated accordingly at each iteration. Besides often finite element method is implemented where equations are formulated for a single element. When mesh is fine enough, constant viscosity on the element is a valid assumption.
} 


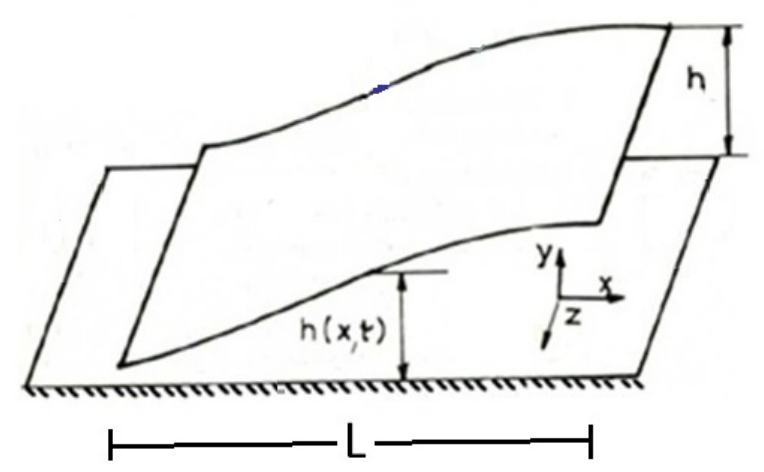

Figure B.2: Thin Lubricant Film, 3]

then Eq. (B.16) in the absence of body forces can be reduced to

$$
\frac{\partial p}{\partial X_{i}}=\mu\left(\frac{1}{3} u_{j, i j}+u_{i, j j}\right)
$$

For the thin lubrication film depicted in Fig. (2.1), the following orders of magnitude apply

$$
O(x, z)=L, O(y)=h, O(u, w)=U, O(v)=\frac{h}{L} U
$$

where due to the thinness of the lubricant film

$$
\frac{h}{L} \ll 1
$$

which further reduces Eq. (B.19) to

$$
\begin{array}{r}
\frac{\partial p}{\partial x}=\mu \frac{\partial^{2} u}{\partial y^{2}} \\
\frac{\partial p}{\partial y}=0 \\
\frac{\partial p}{\partial z}=\mu \frac{\partial^{2} w}{\partial y^{2}}
\end{array}
$$

Eq. (B.23) suggests that the pressure is uniform across the film thickness. Recall that 
for convenience we assigned $X_{1}=x, X_{2}=y, X_{3}=z$, and $u_{1}=u, u_{2}=v, u_{3}=w$. Integration of Eqs. B.22, B.24 subject to the following boundary conditions

$$
\begin{aligned}
& y=0: u(0)=U_{1} ; v(0)=V_{1} ; w(0)=W_{1} \\
& y=h(x, z, t): u(h)=U_{2} ; v(h)=V_{2} ; w(h)=W_{2}
\end{aligned}
$$

yields the following expressions for the velocity profiles

$$
\begin{array}{r}
u=\frac{1}{2 \mu} \frac{\partial p}{\partial x}(y-h) y+U_{1} \frac{h-y}{h}+U_{2} \frac{y}{h} \\
w=\frac{1}{2 \mu} \frac{\partial p}{\partial z}(y-h) y+W_{1} \frac{h-y}{h}+W_{2} \frac{y}{h}
\end{array}
$$

It should be noted that Eqs. (B.27 \& B.28) are valid only for laminar and inertialess flow regime regardless of the compressibility of the lubricant/flow. Equations (B.25) and (B.26) are insufficient to formulate the lubrication problem. Therefore, another relationship is required. The continuity equation, which is the statement of the conservation of mass in an elemental volume for a compressible fluid can be used for this purpose. This equation is given by

$$
\frac{\partial \rho}{\partial t}+\frac{\partial(\rho u)}{\partial x}+\frac{\partial(\rho v)}{\partial y}+\frac{\partial(\rho w)}{\partial z}=0
$$

If we assume a compressible isothermal fluid film, which obeys the perfect gas laws, then

$$
p=\Im \rho
$$

which allows Eq. (B.29) to be rewritten as

$$
\frac{\partial(p v)}{\partial y}=-\left(\frac{\partial p}{\partial t}+\frac{\partial(p u)}{\partial x}+\frac{\partial(p w)}{\partial z}\right)
$$


Integrating Eq. (B.31) across the fluid film yields

$$
\begin{gathered}
\int_{0}^{h} \frac{\partial(p v)}{\partial y} d y=\left.p v\right|_{0} ^{h}=p\left(V_{2}-V_{1}\right) \\
p\left(V_{2}-V_{1}\right)=-\int_{0}^{h(x, z, t)}\left[\frac{\partial p}{\partial t}+\frac{\partial(p u)}{\partial x}+\frac{\partial(p w)}{\partial z}\right] d y
\end{gathered}
$$

In order to perform the above integration, it is necessary to place the derivatives with respect to $x$ and $z$ outside the integration sign. To accomplish this, the Leibniz rule for differentiating under the integral sign when the limits of integration are a function of the current variable itself is used. If

$$
I(\alpha(x), \beta(x), x)=\int_{\alpha(x)}^{\beta(x)} f(x, y) d y
$$

then

$$
\frac{d I}{d x}=\int_{\alpha(x)}^{\beta(x)} \frac{\partial f(x, y)}{\partial x} d y+f(x, \beta(x)) \frac{\partial \beta(x)}{\partial x}-f(x, \alpha(x)) \frac{\partial \alpha(x)}{\partial x}
$$

thus

$$
\begin{gathered}
\int_{0}^{h} \frac{\partial(p u)}{\partial x} d y=\frac{\partial}{\partial x} \int_{0}^{h}(p u) d y-\left.(p u)\right|_{y=h} \frac{\partial h}{\partial x}=\frac{\partial}{\partial x} \int_{0}^{h}(p u) d y-p U_{2} \frac{\partial h}{\partial x} \\
\int_{0}^{h} \frac{\partial(p w)}{\partial z} d y=\frac{\partial}{\partial z} \int_{0}^{h}(p w) d y-\left.(p w)\right|_{y=h} \frac{\partial h}{\partial z}=\frac{\partial}{\partial z} \int_{0}^{h}(p w) d y-p W_{2} \frac{\partial h}{\partial z}
\end{gathered}
$$

Therefore, integrating Eq. B.33) results in

$$
p\left(V_{2}-V_{1}\right)+\frac{\partial p}{\partial t} h+\frac{\partial}{\partial x} \int_{0}^{h}(p u) d y+\frac{\partial}{\partial z} \int_{0}^{h}(p w) d y=p U_{2} \frac{\partial h}{\partial x}+p W_{2} \frac{\partial h}{\partial z}
$$


It is useful to define the volumetric unit flow rates in the $x$ and $z$ directions as

$$
\begin{aligned}
& q_{x}=\int_{0}^{h} u d y=-\frac{h^{3}}{12 \mu} \frac{\partial p}{\partial x}+\left(U_{1}+U_{2}\right) \frac{h}{2} \\
& q_{z}=\int_{0}^{h} w d y=-\frac{h^{3}}{12 \mu} \frac{\partial p}{\partial z}+\left(W_{1}+W_{2}\right) \frac{h}{2}
\end{aligned}
$$

Eq. (B.33) can then be expressed as

$$
p\left(V_{2}-V_{1}\right)+\frac{\partial p}{\partial t} h+\frac{\partial\left(p q_{x}\right)}{\partial x}+\frac{\partial\left(p q_{z}\right)}{\partial z}=p U_{2} \frac{\partial h}{\partial x}+p W_{2} \frac{\partial h}{\partial z}
$$

Then substituting Eqs. (B.39 \& B.40) into Eq. (B.41) and rearranging the terms results in

$$
\begin{gathered}
\frac{1}{6 \mu}\left[\frac{\partial}{\partial x}\left(p h^{3} \frac{\partial p}{\partial x}\right)+\frac{\partial}{\partial z}\left(p h^{3} \frac{\partial p}{\partial z}\right)\right]=2 p\left(V_{2}-V_{1}\right)+2 \frac{\partial p}{\partial t} h \\
+h\left(\frac{\partial}{\partial x}\left[p\left(U_{2}+U_{1}\right)\right]+\frac{\partial}{\partial z}\left[p\left(W_{2}+W_{1}\right)\right]\right) \\
+p\left(U_{1}-U_{2}\right) \frac{\partial h}{\partial x}+p\left(W_{1}-W_{2}\right) \frac{\partial h}{\partial z}
\end{gathered}
$$

The above equation represents the general traditional three-dimensional Reynolds equation as applied to an isothermal compressible fluid film. This equation is nonlinear in terms of pressure. Often Eq. B.42 reduces to simpler forms in cases such as, perfectly axially aligned bearings $\left(\frac{\partial}{\partial z}=0\right)$, or stationary bearings $\left(U_{1}=W_{1}=\right.$ $\left.W_{2}=0\right)$. 


\section{Appendix $\mathrm{C}$}

\section{Coefficient Identification for a}

\section{$\mathrm{CCO}$}

To identify the linear coefficients of a SFD operating in a CCO, full matrix coefficients based on a KCM model can be assumed

$$
\begin{aligned}
{\left[\begin{array}{ll}
M_{x x} & M_{x y} \\
M_{y x} & M_{y y}
\end{array}\right]\left\{\begin{array}{l}
\ddot{X} \\
\ddot{Y}
\end{array}\right\}+\left[\begin{array}{ll}
C_{x x} & C_{x y} \\
C_{y x} & C_{y y}
\end{array}\right]\left\{\begin{array}{l}
\dot{X} \\
\dot{Y}
\end{array}\right\} } \\
+\left[\begin{array}{ll}
K_{x x} & K_{x y} \\
K_{y x} & K_{y y}
\end{array}\right]\left\{\begin{array}{c}
X \\
Y
\end{array}\right\}=\left\{\begin{array}{c}
F_{X} \\
F_{Y}
\end{array}\right\}
\end{aligned}
$$

Considering two points on the CCO, the points "1" and "2" are

$$
\left\{\begin{array}{l}
X_{1} \\
Y_{1}
\end{array}\right\}=\left\{\begin{array}{l}
r \\
0
\end{array}\right\}, \quad\left\{\begin{array}{l}
X_{2} \\
Y_{2}
\end{array}\right\}=\left\{\begin{array}{l}
0 \\
r
\end{array}\right\}
$$




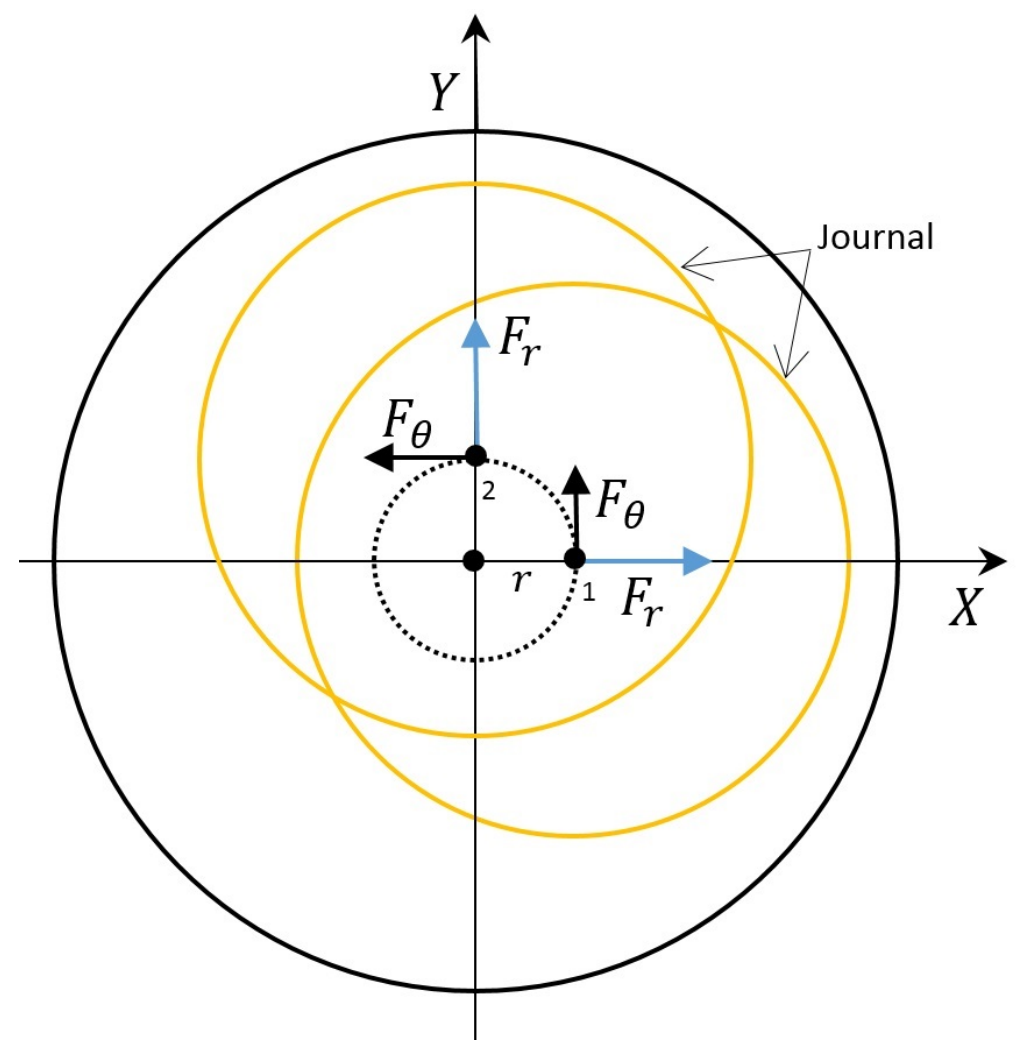

Figure C.1: Circular centered orbit, CCO

where we have

$$
\begin{aligned}
& \left\{\begin{array}{c}
\dot{X}_{1} \\
\dot{Y}_{1}
\end{array}\right\}=\left\{\begin{array}{c}
0 \\
r \Omega
\end{array}\right\}, \quad\left\{\begin{array}{c}
\ddot{X}_{1} \\
\ddot{Y}_{1}
\end{array}\right\}=\left\{\begin{array}{c}
-r \Omega^{2} \\
0
\end{array}\right\} \\
& \left\{\begin{array}{c}
\dot{X}_{2} \\
\dot{Y}_{2}
\end{array}\right\}=\left\{\begin{array}{c}
-r \Omega \\
0
\end{array}\right\}, \quad\left\{\begin{array}{c}
\ddot{X}_{2} \\
\ddot{Y}_{2}
\end{array}\right\}=\left\{\begin{array}{c}
0 \\
-r \Omega^{2}
\end{array}\right\}
\end{aligned}
$$

Since CCO and SFD are symmetric, the radial and tangential forces maintain the same amplitude everywhere on its circumference, as depicted in Fig. (C.1). Thus, we have

$$
\left\{\begin{array}{c}
F_{X 1} \\
F_{Y 1}
\end{array}\right\}=\left\{\begin{array}{l}
F_{r} \\
F_{\theta}
\end{array}\right\}, \quad\left\{\begin{array}{c}
F_{X 2} \\
F_{Y 2}
\end{array}\right\}=\left\{\begin{array}{c}
-F_{\theta} \\
F_{r}
\end{array}\right\}
$$


If we replace displacement, velocity, acceleration, and force vectors related to point "1" in Eq. C.1, we have

$$
\begin{array}{r}
{\left[\begin{array}{cc}
M_{x x} & M_{x y} \\
M_{y x} & M_{y y}
\end{array}\right]\left\{\begin{array}{c}
-r \Omega^{2} \\
0
\end{array}\right\}+\left[\begin{array}{cc}
C_{x x} & C_{x y} \\
C_{y x} & C_{y y}
\end{array}\right]\left\{\begin{array}{c}
0 \\
r \Omega
\end{array}\right\}} \\
+\left[\begin{array}{cc}
K_{x x} & K_{x y} \\
K_{y x} & K_{y y}
\end{array}\right]\left\{\begin{array}{c}
r \\
0
\end{array}\right\}=\left\{\begin{array}{c}
F_{r} \\
F_{\theta}
\end{array}\right\}
\end{array}
$$

which results in

$$
\begin{aligned}
& -M_{x x} \Omega^{2}+C_{x y} \Omega+K_{x x}=\frac{F_{r}}{r} \\
& -M_{y x} \Omega^{2}+C_{y y} \Omega+K_{y x}=\frac{F_{\theta}}{r}
\end{aligned}
$$

Similarly, for point " 2 ", we have

$$
\begin{gathered}
{\left[\begin{array}{cc}
M_{x x} & M_{x y} \\
M_{y x} & M_{y y}
\end{array}\right]\left\{\begin{array}{c}
0 \\
-r \Omega^{2}
\end{array}\right\}+\left[\begin{array}{cc}
C_{x x} & C_{x y} \\
C_{y x} & C_{y y}
\end{array}\right]\left\{\begin{array}{c}
-r \Omega \\
0
\end{array}\right\}} \\
+\left[\begin{array}{cc}
K_{x x} & K_{x y} \\
K_{y x} & K_{y y}
\end{array}\right]\left\{\begin{array}{l}
0 \\
r
\end{array}\right\}=\left\{\begin{array}{c}
-F_{\theta} \\
F_{r}
\end{array}\right\}
\end{gathered}
$$

which results in

$$
\begin{aligned}
& -M_{x y} \Omega^{2}-C_{x x} \Omega+K_{x y}=-\frac{F_{\theta}}{r} \\
& -M_{y y} \Omega^{2}-C_{y x} \Omega+K_{y y}=\frac{F_{r}}{r}
\end{aligned}
$$


Eqs. C.7 C.11) are equal for any whirling speed and similarly the equality between Eqs. C.8 C.10 holds for all $\Omega$ values so we can conclude that

$$
\begin{aligned}
& -M_{y y} \Omega^{2}-C_{y x} \Omega+K_{y y}=-M_{x x} \Omega^{2}+C_{x y} \Omega+K_{x x} \\
& M_{x y} \Omega^{2}+C_{x x} \Omega-K_{x y}=-M_{y x} \Omega^{2}+C_{y y} \Omega+K_{y x}
\end{aligned}
$$

which results in

$$
\begin{aligned}
& M_{x x}=M_{y y}=M, M_{x y}=-M_{y x}=m \\
& C_{x x}=C_{y y}=C, C_{x y}=-C_{y x}=c \\
& K_{x x}=K_{y y}=K, K_{x y}=-K_{y x}=k \\
& {\left[\begin{array}{ll}
M_{x x} & M_{x y} \\
M_{y x} & M_{y y}
\end{array}\right]=\left[\begin{array}{cc}
M & m \\
-m & M
\end{array}\right]} \\
& {\left[\begin{array}{cc}
C_{x x} & C_{x y} \\
C_{y x} & C_{y y}
\end{array}\right]=\left[\begin{array}{cc}
C & c \\
-c & C
\end{array}\right]} \\
& {\left[\begin{array}{cc}
K_{x x} & K_{x y} \\
K_{y x} & K_{y y}
\end{array}\right]=\left[\begin{array}{cc}
K & k \\
-k & K
\end{array}\right]}
\end{aligned}
$$

which are asymmetric full coefficient matrices with principle and cross-coupled terms. 